\title{
Frantz FANON
}

Psychiatre et militant de l'indépendance algérienne dans le FLN

\section{[1961] (2002)}

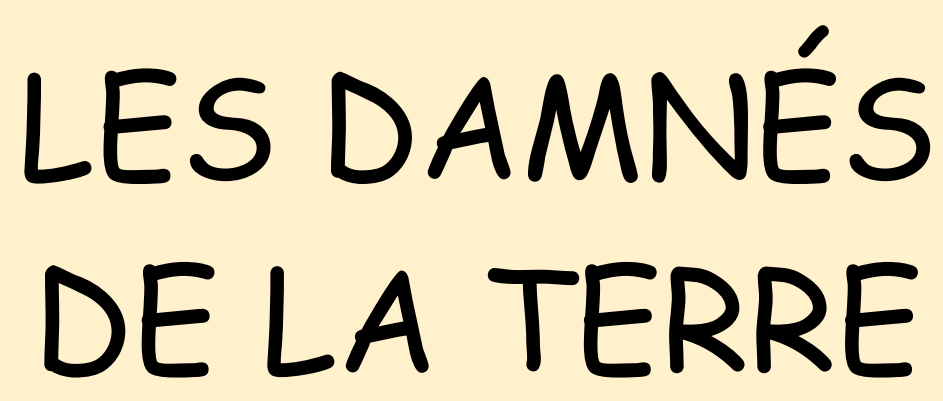

Un document produit en version numérique par Émilie Tremblay, bénévole, Doctorante en sociologie à l'Université de Montréal

Courriel: emiliet82@yahoo.fr

Page web dans Les Classiques des sciences sociales.

Dans le cadre de: "Les classiques des sciences sociales"

Une bibliothèque numérique fondée et dirigée par Jean-Marie Tremblay, professeur de sociologie au Cégep de Chicoutimi

Site web: http://classiques.uqac.ca/

Une collection développée en collaboration avec la Bibliothèque

Paul-Émile-Boulet de l'Université du Québec à Chicoutimi

Site web: http://bibliotheque.ugac.ca/ 


\section{Politique d'utilisation de la bibliothèque des Classiques}

Toute reproduction et rediffusion de nos fichiers est interdite, même avec la mention de leur provenance, sans l'autorisation formelle, écrite, du fondateur des Classiques des sciences sociales, Jean-Marie Tremblay, sociologue.

Les fichiers des Classiques des sciences sociales ne peuvent sans autorisation formelle:

- être hébergés (en fichier ou page web, en totalité ou en partie) sur un serveur autre que celui des Classiques.

- servir de base de travail à un autre fichier modifié ensuite par tout autre moyen (couleur, police, mise en page, extraits, support, etc...),

Les fichiers (.html, .doc, .pdf, .rtf, .jpg, .gif) disponibles sur le site Les Classiques des sciences sociales sont la propriété des Classiques des sciences sociales, un organisme à but non lucratif composé exclusivement de bénévoles.

Ils sont disponibles pour une utilisation intellectuelle et personnelle et, en aucun cas, commerciale. Toute utilisation à des fins commerciales des fichiers sur ce site est strictement interdite et toute rediffusion est également strictement interdite.

L'accès à notre travail est libre et gratuit à tous les utilisateurs. C'est notre mission.

Jean-Marie Tremblay, sociologue

Fondateur et Président-directeur général, LES CLASSIQUES DES SCIENCES SOCIALES. 


\section{REMARQUE}

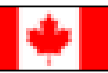

Ce livre est du domaine public au Canada parce qu'une œuvre passe au domaine public 50 ans après la mort de l'auteur(e).

Cette œuvre n'est pas dans le domaine public dans les pays où il faut attendre 70 ans après la mort de l'auteur(e).

Respectez la loi des droits d'auteur de votre pays. 
Du même auteur

Peau noire, masques blancs, Seuil, 1952 ; rééd. Seuil, coll. «Point/Essais », 1971.

Sociologie d'une révolution. L'An $V$ de la révolution algérienne, Librairie François Maspero, coll. « Cahiers libres », 1959 ; rééd. La Découverte, coll. « Redécouverte», 2001.

Pour la révolution africaine, Librairie François Maspero, coll. « Cahiers libres», 1964 ; rééd. La Découverte, coll. « Redécouverte», 2001. 
Cette édition électronique a été réalisée par Émilie Tremblay, bénévole, doctorante en sociologie à l'Université de Montréal

Courriel : emiliet82@yahoo.fr

à partir de :

Frantz FANON

\section{LES DAMNÉS DE LA TERRE.}

Préface de Jean-Paul Sartre (1961); Préface d'Alice Cherki et postface de Mohammed Harbi (2002). Paris: Éditions La Découverte/Poche, 2002, 313 pp. Paris: François Maspero, 1961, 1968.

Polices de caractères utilisée:

Pour le texte: Comic Sans, 12 points.

Pour les citations : Comic Sans, 12 points.

Pour les notes de bas de page : Comic Sans, 10 points.

Édition électronique réalisée avec le traitement de textes Microsoft Word 2008 pour Macintosh.

Mise en page sur papier format : LETTRE US, $8.5^{\prime \prime} \times 11^{\prime \prime}$ )

Édition numérique réalisée le 27 janvier 2011 à Chicoutimi, Ville de Saguenay, Québec.

\section{E Fait avec}

Macintosh 


\section{Frantz FANON}

Psychiatre, intellectuel antillais

et militant de l'indépendance algérienne dans le FLN

\section{LES DAMNÉS DE LA TERRE}

Frantz Fanon

Les damnés de la terre

Préface de Jean-Paul Sartre (1961)

Préface d'Alice Cherki et

postface de Mohammed Harbi (2002)

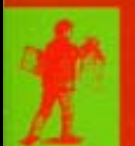

$\frac{8}{8}$

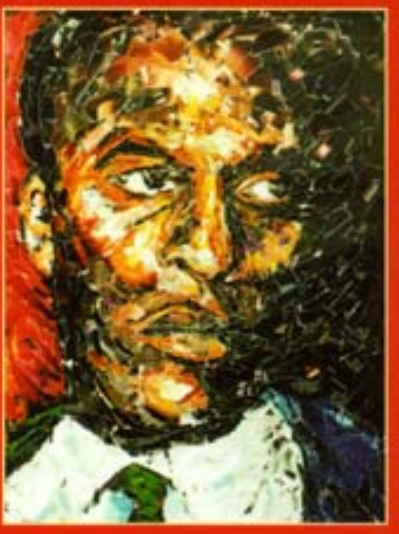

Préface de Jean-Paul Sartre (1961); Préface d'Alice Cherki et postface de Mohammed Harbi (2002). Paris: Éditions La Découverte/Poche, 2002, 313 pp. Paris: François Maspero, 1961, 1968. 


\section{Table des matières}

Préface à l'édition de 2002, par Alice Cherki

Préface à l'édition de 1961, par Jean-Paul Sartre

1. De la violence

De la violence dans le contexte international

2. Grandeur et faiblesses de la spontanéité

3. Mésaventures de la conscience nationale

4. Sur la culture nationale

Fondements réciproques de la culture nationale et des luttes de libération

5. Guerre coloniale et troubles mentaux

Série A

Série B

$\underline{\text { Série } C}$

Série D

De l'impulsivité criminelle du Nord-Africain à la guerre de Libération nationale

Conclusion

Postface à l'édition de 2002, par Mohammed Harbi 
Ce livre a été précédemment publié en 1961 aux éditions François Maspero et en 1968, chez le même éditeur, avec la préface de Jean-Paul Sartre. Une nouvelle édition de cet ouvrage a également été publiée en 1991 par les éditions Gallimard avec une présentation de Gérard Chaliand. 


\section{LES DAMNÉS DE LA TERRE}

\section{Quatrième de couverture}

\section{Retour à la table des matières}

Publié en 1961, à une époque où la violence coloniale se déchaîne avec la guerre d'Algérie, saisi à de nombreuses reprises lors de sa parution aux Éditions François Maspero, le livre Les Damnés de la terre, préfacé par Jean-Paul Sartre, a connu un destin exceptionnel. Il a ser$\mathrm{vi}$ - et sert encore aujourd'hui - d'inspiration et de référence à des générations de militants anticolonialistes. Son analyse du traumatisme du colonisé dans le cadre du système colonial et son projet utopique d'un tiers monde révolutionnaire porteur d'un « homme neuf » restent un grand classique du tiers-mondisme, l'œuvre capitale et le testament politique de Frantz Fanon.

Dans cette nouvelle édition, la préface d'Alice Cherki, psychiatre et psychanalyste, auteur du Portrait de Franc Fanon (Seuil, 2000), et la postface de Mohammed Harbi, combattant de la première heure pour la libération de son pays et historien de l'Algérie contemporaine, auteur de Une vie debout. Mémoires politiques 1945-1962 (La Découverte, 2001), restituent l'importance contemporaine de la pensée de Frantz Fanon. 
«Faire sauter le monde colonial est désormais une image d'action très claire, très compréhensible et pouvant être reprise par chacun des individus constituant le peuple colonisé. »

\section{Frantz Fanon}

Préface de

Jean-Paul Sartre (1961)

Préface d'Alice Cherkiet postface de Mohammed Harbi (2002)

Frantz Fanon (1925-1961), psychiatre antillais, militant de l'indépendance algérienne au sein du FLN, est notamment l'auteur de Peau noire, masques blancs (Seuil), de L'An $V$ de la révolution algérienne ( $L a$ Découverte) et de Pour la révolution africaine (La Découverte).

En couverture: Frantz Fanon de Mustapha Boutadjine, Paris, 2001. Graphismecollage, $135 \mathrm{~cm} \times 105 \mathrm{~cm}$. Collection privée. Extrait du thème: « Black is toujours beautiful », galerie d'art Arcima, Paris. Avec l'aimable autorisation de l'artiste. 


\title{
LES DAMNÉS DE LA TERRE \\ Préface à l'édition de 2002
}

\author{
Par Alice Cherki
}

\section{Retour à la table des matières}

Le livre Les Damnés de la terre paraît fin novembre 1961 aux Éditions François Maspero alors que son auteur, Frantz Fanon, atteint d'une leucémie, lutte contre la mort dans la clinique de Bestheda, près de Was-

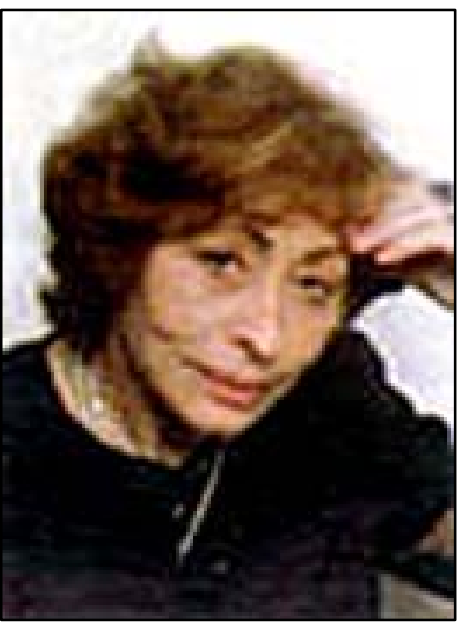
hington, aux États-Unis. Imprimé dans des conditions difficiles de semi-clandestinité pour ne pas être saisi à la sortie du marbre, le livre est interdit dès sa diffusion sous le chef d'inculpation d' " atteinte à la sécurité intérieure de l'État». Cela s'était déjà produit pour le précédent livre de Fanon édité également par Maspero en 1959, L'An $V$ de la révolution algérienne, et pour un certain nombre d'autres ouvrages relatifs à la guerre d'Algérie (comme Le Refus de Maurice Maschino, Le Déserteur de Maurienne ou, antérieurement, La Question de Henri Alleg). Ces interdictions étaient d'usage à l'époque.

Toutefois, le livre circule et la presse lui donne un large écho. Par un acheminement compliqué, via la Tunisie, Fanon en recevra le 3 décembre un exemplaire, ainsi que des coupures de presse, dont un long 
article de Jean Daniel paru dans L'Express du 30 novembre, plutôt élogieux. À la lecture qui lui en est faite, Fanon réplique: «Certes, mais ce n'est pas cela qui me rendra ma moelle. » Fanon meurt quelques jours plus tard, le 8 décembre 1961. Il avait trente-six ans.

En effet, il est né en 1925 à Fort-de-France, en Martinique, dans une famille de la petite bourgeoisie aisée. Enfant d'une fratrie nombreuse, il évolue dans un monde de vieille colonie où il n'est pas encore d'usage de s'interroger sur l'esclavage. Pourtant, très jeune, Fanon s'engage dans les Forces gaullistes, [6] le bataillon V, regroupant les volontaires des Caraïbes. C'est au cours de cet engagement qu'il acquiert sa culture de résistance, mais il y fait également l'expérience du racisme banal, quotidien. Démobilisé, avec la croix de guerre (qui lui avait été décernée par le futur général Salan, dont il avait coutume de dire que c'était la seule chose qu'il avait en commun avec lui), il revient en Martinique en 1945, passe son bac et fréquente Aimé Césaire (pour lequel il a une grande admiration mais dont il ne partage déjà pas les options politiques). Césaire, à l'époque, choisit de considérer la Martinique comme un département français.

Fanon se retrouve très rapidement en France pour poursuivre ses études de médecine, à Lyon. Parallèlement à ces études, il se passionne pour la philosophie, l'anthropologie, le théâtre, et s'engage tôt dans la spécialisation en psychiatrie. Dans le même temps, il n'adhère à aucun parti politique mais participe à toute la mouvance anticolonialiste et contribue à la rédaction d'un petit périodique, Tam Tam, destiné aux étudiants originaires des colonies. Et, surtout, il écrit un premier article dans la revue Esprit en 1952, "Le syndrome nord-africain», dans lequel il s'interroge sur l'ouvrier nord-africain, exilé, souffrant d'être un « homme mort quotidiennement» qui, coupé de ses origines et coupé de ses fins, devient un objet, une chose jetée dans le grand fracas.

À l'hôpital psychiatrique de Saint-Alban, où il restera quinze mois, Fanon fait une rencontre essentielle, celle de François Tosquelles, psychiatre d'origine espagnole et militant antifranquiste. Ce fut pour lui une formation déterminante, et sur le plan de la psychiatrie et sur celui de ses futurs engagements. Il y trouve le point de rencontre où 
I'aliénation est interrogée dans tous ses registres, au lieu de jonction du somatique et du psychique, de la structure et de l'histoire. En 1953, il passe le médicat des hôpitaux psychiatriques et est alors nommé à I' hôpital psychiatrique de Blida, en Algérie. Son premier livre, Peau noire, masques blancs, était déjà paru aux Éditions du Seuil, grâce à Francis Jeanson, en 1952.

En Algérie, il se trouve confronté non seulement à la psychiatrie classique des asiles, mais également à la théorie des [7] psychiatres de l'école d'Alger sur le primitivisme des indigènes. Il découvre, de proche en proche, la réalité coloniale de l'Algérie de l'époque. Il mettra dans un premier temps toute son énergie à transformer les services dont il a la responsabilité en y introduisant la « social-thérapie » pratiquée avec Tosquelles. Il n'aura de cesse de transformer ainsi le rapport des soignants aux aliénés, avec les Européens mais également avec les « indigènes » musulmans, cherchant à restaurer leurs référents culturels, leur langue, l'organisation de leur vie sociale, tout ce qui pouvait faire sens. Cette petite révolution psychiatrique est reconnue aussi bien par le personnel soignant - pour la plupart engagé politiquement - que par des militants de la région. La réputation de Fanon s'étend. Nous sommes déjà en 1955 et la guerre d'Algérie a commencé.

Fanon ne comprend pas l'aveuglement du gouvernement socialiste français devant le désir d'indépendance des Algériens et ses positions anticolonialistes sont de plus en plus connues. Il sera contacté par le mouvement « Amitiés algériennes», association humanitaire destinée à apporter un soutien matériel aux familles des détenus politiques, dirigée en fait par des militants nationalistes en liaison avec les combattants ayant pris le maquis près de Blida. La première demande qui lui est faite est celle de prendre en charge des maquisards souffrant de troubles psychiques.

C'est ainsi, par capillarité entre psychiatrie et engagement politique, que Fanon s'engage dans la lutte des Algériens pour leur indépendance. Fin 1956, il démissionne de son poste de médecin psychiatre, dans une lettre ouverte au résident général Robert Lacoste où il écrit 
qu'il lui est impossible de vouloir coûte que coûte désaliéner des individus, les « remettre à leur place dans un pays où le non-droit, l'inégalité et le meurtre sont érigés en principes législatifs, où l'autochtone, aliéné permanent dans son propre pays, vit dans un état de dépersonnalisation absolu». Fanon est expulsé d'Algérie.

Il passe ensuite trois mois en France, au premier trimestre 1957, séjour au cours duquel il ne trouve pas d'écho à sa conviction [8] que l'indépendance de l'Algérie est inéluctable. Aidé par la fédération de France du FLN, il rejoint Tunis où se met en place l'organisation extérieure du mouvement de libération nationale. La rupture est consommée.

Fanon poursuivra à Tunis une double activité, à la fois psychiatrique et politique. Il deviendra membre de l'équipe du journal du FLN, El Moudjahid. Il assistera de l'intérieur à toutes les contradictions du Front de libération nationale, y compris aux querelles grandissantes entre les représentants politiques et l'armée. Souvent déçu, il restera néanmoins un défenseur de la lutte de libération algérienne et un psychiatre constamment novateur. Il s'intéressera de plus en plus à I'Afrique subsaharienne et sera nommé par le gouvernement provisoire de la République algérienne ambassadeur itinérant en Afrique noire, fin 1959. C'est l'année des indépendances africaines. Fanon sera véritablement un itinérant, se dépensant sans compter du Ghana au Cameroun, de I'Angola au Mali, afin de promouvoir un combat pour une véritable indépendance. Il envisage même la possibilité d' un front qui partirait du Mali pour traverser le Sahara et rejoindre les combattants algériens.

Mais en décembre 1960, au cours d'un séjour à Tunis, Fanon découvre qu'il est atteint d'une leucémie myéloïde. Il lui reste un an à vivre, au cours duquel il écrira Les Damnés de la terre.

Ce livre - dont le titre fut le seul qu'il choisit lui-même et non ses éditeurs - fut rédigé par un homme qui se savait condamné par un mal dont il n'ignorait pas, en tant que médecin, qu'il était alors incurable. 
Dans une véritable course contre la montre et la mort, Fanon veut faire passer un dernier message. À qui ? Aux déshérités, qui ne sont plus essentiellement les prolétaires des pays industrialisés de la fin du $X I X{ }^{e}$ siècle chantant « Debout les damnés de la terre, debout les forçats de la faim». Les damnés de la terre auxquels Fanon s'adresse sont les déshérités des pays pauvres qui veulent réellement la terre et du pain, alors qu'à l'époque la classe ouvrière du monde occidental, souvent raciste et manifestement [9] ignorante des populations d'outre-mer, témoigne d'une relative indifférence au sort des colonies dont elle tire indirectement bénéfice.

Ni traité d'économie, ni essai de sociologie voire de politique, cet ouvrage est un appel et même un cri d'alarme sur l'état et le devenir des pays colonisés. Comme dans toute son œuvre, Fanon y met en tension politique, culture et individu, prenant en compte les effets de la domination économique, politique et culturelle sur le dominé. Son analyse insiste sur les conséquences de l'asservissement non seulement des peuples mais des sujets, et sur les conditions de leur libération, qui est avant tout une libération de l'individu, une « décolonisation de l'être».

Les Damnés de la terre est donc le dernier livre de Frantz Fanon. Il avait déjà écrit, en 1952 à l'âge de vingt-cinq ans, Peau noire, masques blancs et, en 1959, L'An $V$ de la Révolution algérienne qui fut alors I'un des premiers livres édités par François Maspero. Il avait également produit de nombreux articles: «Le syndrome nordafricain », déjà évoqué, des contributions de psychiatrie et notamment «Racisme et culture » au premier congrès des écrivains noirs en 1956, puis «Culture et nation» au deuxième congrès des écrivains noirs à Rome en 1959. Dans tous ces textes, le développement de l'argumentation est fondé non sur le théorique mais sur le vécu, point de départ du développement de sa pensée. Déjà dans Peau noire, masques blancs, la réflexion sur le racisme était rattachée à la domination de certaines cultures décrétée unilatéralement : il ne s'agit pas d'un accident, d'un caprice psychologique, mais d'un système culturel d'oppression à l'œuvre également dans la situation coloniale. Lutter contre le racisme est 
vain si on n'éclaire pas les effets de l'oppression exercée par la culture dominante, oppression qui atteint les communautés, le politique et la culture mais aussi l'être psychique.

Dans Les Damnés de la terre se poursuit cette interrogation sur l'aliénation par un monde dominant qui subvertit et altère aussi bien les collectivités que les sujets dans leur devenir personnel. Le livre reprend, en les radicalisant dans le cadre du [10] combat politique, les données des rapports dominant/dominé et les conditions de libération, alliant au politique et à la culture la libération du sujet. Les deux derniers chapitres sont d'ailleurs consacrés l'un à la culture et son rapport avec la construction de la nation et l'autre aux troubles psychiques traumatiques engendrés de part et d'autre par la guerre d'Algérie.

Fanon écrit à partir de son expérience singulière, depuis I'histoire immédiate, de sa plongée dans cette histoire, expérience qu'il lui est nécessaire d'élaborer et de transmettre. L'écriture même suit ce mouvement: les différents thèmes qui composent les cinq chapitres du livre sont disposés comme des fragments, comme les strophes d'un poème auxquelles se mêlent des temps $d$ 'analyse rigoureuse mais toujours écrite dans une langue qui, comme le disait lui-même le jeune Fanon à propos de son premier livre Peau noire, masques blancs, cherche à produire, au-delà des significations, une compréhension qui n'est pas liée au seul maniement du concept.

On a pu et on peut reprocher à Fanon d'avoir mélangé les genres et les niveaux de discours - analyse politique, culturelle et psychologique -, d'avoir transposé du champ de son expérience de psychiatre confronté à l'aliénation mentale des données qui ne conviendraient pas au champ du politique. On a pu lui reprocher son style, en le qualifiant de lyrique et de prophétique. Mais, paradoxalement, c'est cela qui fait la modernité de Fanon. Psychiatre, son expérience des subjectivités en souffrance le plaçait au contact direct des déshérités.

On lui a reproché aussi d'avoir insisté sur la violence. Or Fanon savait d'expérience les effets de la violence exercée contre l'individu: 
celui-ci n'a d'autre recours pour lui-même que la pétrification dépersonnalisante ou l'envahissement par une terrible violence pulsionnelle qu'il va mettre en acte de façon erratique. Cette violence, au lieu d'être niée, doit être organisée en lutte de libération qui permet le dépassement. Dans «Racisme et culture», Fanon concluait ainsi son intervention: "La culture spasmée et rigide de l'occupant, libérée, s'ouvre enfin à la culture du peuple devenu réellement frère. Les deux [11] cultures peuvent s'affronter, s'enrichir. [...] L'universalité réside dans cette décision de prise en charge du relativisme réciproque de cultures différentes une fois exclu irréversiblement le statut colonial. » Et dans Peau noire, masques blancs, il indiquait également ce dépassement entre le monde noir et le monde blanc: « Tous deux ont à s'écarter des voix inhumaines qui furent celles de leurs ancêtres respectifs afin que naisse une véritable communication. "Cette vision de dépassement se retrouve, même si elle s'est radicalisée entre-temps dans le combat politique, dans Les Damnés de la terre.

La belle préface de Sartre à ce livre, que Fanon avait souhaitée, fut, semble-t-il, davantage lue au cours des années que le corps du texte. Et pourtant, d'une certaine façon, elle détourne les préoccupations et le ton de Fanon. Elle s'adresse essentiellement aux Européens, introduisant une première discordance entre ce texte et celui qu'il présente. Fanon, lui, s'adresse à tous les autres et leur parle effectivement d'un avenir où serait dépassée la «peur de l'autre». Et, surtout, cette préface radicalise l'analyse de Fanon sur la violence. En effet, Sartre justifie la violence alors que Fanon l'analyse, ne la promeut pas comme une fin en soi mais y voit un passage obligé. De ce fait, l'écrit de Sartre prend par instants des accents d'incitation à la criminalité. Des phrases telles que: «Lisez Fanon: vous saurez que, dans le temps de leur impuissance, la folie meurtrière est l'inconscient collectif des colonisés», ou encore : « Abattre un Européen, c'est faire d'une pierre deux coups, supprimer en même temps un oppresseur et un opprimé : restent un homme mort et un homme libre », réduisent la portée des propositions de Fanon, parce qu'elles semblent justifier non plus la violence, mais le meurtre réel individuel. On est dans la cri- 
minalité et non plus dans cette violence inhérente à tout être humain, qui est appel pour advenir comme être dans un possible de soi-même. Fanon, en lisant la préface de Sartre, ne fit aucun commentaire; il resta même, contrairement à son habitude, extrêmement silencieux. Néanmoins, il écrivit à François Maspero qu'il espérait avoir, le moment venu, la possibilité de s'expliquer.

[12]

Les Damnés de la terre, considéré comme un livre phare des années soixante-dix, essentiellement lié au tiers-mondisme et dont les avancées politiques étaient alors privilégiées au détriment de son interrogation insistante sur les fondements de l'aliénation de l'opprimé où qu'il se trouve, tomba ensuite dans l'oubli et, avec lui, l'ensemble de l'œuvre de Fanon considérée comme datée. Ses audaces politiques furent désignées comme obsolètes - puisque liées à une époque de la décolonisation dite révolue - et porteuses d'un espoir déçu par les faits. Fanon n'avait-il pas surestimé la force des masses paysannes dans les luttes de libération? Il se trouve que, dans le contexte politique de la lutte algérienne à l'époque, ce sont bien majoritairement les paysans qui constituaient les combattants. N'oublions pas que Fanon écrit une expérience historique ponctuelle. Et que, pour lui, le dynamisme du paysan peut aussi bien, comme il l'explique dans « Grandeur et faiblesses de la spontanéité » (chapitre 2 des Damnés de la terre), accompagner la réaction que la révolution.

N'avait-il pas sous-estimé la force du religieux? En fait, la lutte de libération algérienne qu'il avait rejointe ne se présentait pas comme une révolution islamique et ralliait différents courants - la plateforme du congrès de la Soummam, en 1956, malgré les contradictions de ses inspirateurs, ne mettait pas en avant une centralité religieuse mais plutôt un recours à la pluralité. L'appel de Fanon aux pays en voie de décolonisation à inventer, à créer un homme nouveau n'a-t-il pas été infirmé par le devenir des pays d'Afrique? L'évolution géopolitique ultérieure ne constitue-t-elle pas un démenti à tous ses espoirs? En fait, cette évolution a plutôt confirmé le bien-fondé de ses mises en 
garde (dans le chapitre «Mésaventures de la conscience nationale ») face à un devenir qu'il redoutait.

Fanon analysait une réalité contingente et son livre ne peut être perçu comme « déphasé » que si on le limite au contexte de son époque au lieu de l'entendre comme un appel à ce qui serait possible. Que ses espoirs ne se soient pas concrétisés rendent-ils erronée la réalité à partir de laquelle il les exprimait? On le sait bien, cette réalité, y compris celle de la violence, ne se dit plus [13] aujourd'hui en termes d'oppression coloniale ou d'avenir du tiers monde, mais en termes d'accroissement des inégalités, d'écart grandissant entre le Nord et le Sud, d'exclusion, de réduction des sujets à des objets.

Quarante ans après la décolonisation et la guerre d'Algérie, dans un monde que l'on a vu s'avancer vers le diktat de la mondialisation économique, cette réalité s'écrit et se profile quotidiennement dans le rapport Sud/Nord: est mise en place la corruption organisée, institutionnalisée par les gouvernements des pays d'Afrique et instaurée par les grandes sociétés pétrolières, pharmaceutiques et autres du monde développé. Dans le même temps, et au nom de la non-ingérence mais surtout d'un impérialisme économique à maintenir, ce même monde s'est montré indifférent à la mise à mal de tout mouvement libérateur d'aspiration démocratique, de toute accession des peuples au gouvernement d'eux-mêmes dont Fanon rêvait et pour lesquels, de psychiatre engagé, il était devenu militant de la cause des peuples opprimés.

Mais cette réalité ne concerne pas seulement les pays dits «en voie de développement ». Elle concerne également l'accroissement des inégalités dans notre monde dit « développé », qui inscrit la nécessité de la précarité et du chômage pour les plus déshérités, quitte à lui donner une place topique et non pas utopique: cette place est celle de l'exclusion. Fanon la réfutait, car il ne voulait pas d'une vie pour chacun qui soit la «mort à bout touchant», une survie au quotidien, qui fait percevoir la vie "non comme épanouissement ou développement d'une fécondité essentielle mais comme lutte permanente contre une mort atmosphérique». Fanon désirait que tout homme soit sujet de son histoire et acteur du politique. 
Du Rwanda à la Bosnie, de l'Afghanistan au Moyen-Orient, sans épargner l'Amérique ni l'Europe, s'étend un monde fracturé, mis à feu et à sang, où les violences succèdent aux violences, où les États s'étonnent et s'indignent de ce qu'ils provoquent, la violence des populations engendrant un cycle infernal et déshumanisant, déstructurant la pensée, la vie et [14] l'avenir des générations du XXI siècle, sur le plan de l'être individuel comme sur le plan collectif.

On reparle aujourd'hui de la guerre d'Algérie: on la nomme enfin alors que pendant trente-cinq ans elle fut appelée "événements». On réactualise et on dénonce la torture. Mais nombre des écrits actuels renvoient dos à dos les atrocités des deux camps alors en conflit, au détriment de l'analyse de la dissymétrie des forces. Ce rapport de forces de deux mondes coupés l'un de l'autre, excluant tout dialogue, que Fanon avait analysé pour son époque, n'est-il pas encore aujourd'hui à l'œuure dans maintes régions du monde? Quand les sociétés et les États développés s'étonnent de l'irruption de la violence au sein même de leurs territoires, l'indignation ne vient-elle pas prendre la place de l'entendement? Entendre ceci : que se joue-t-il quand aucun pacte ne se tisse entre ces deux mondes, quand tout espace de médiation par la parole se referme et que le monde le plus fort se prétend propriétaire du lieu de l'autre, que ce lieu soit territorial, culturel ou psychique? C'est justement la prévision de ce monde qui avait alarmé Fanon et qui l'avait poussé à écrire Les Damnés de la terre.

Il avait également perçu les conséquences traumatiques des guerres, y compris de libération, aux séquelles interminables, conduisant à la répétition de la violence et aux régressions ethniques et identitaires. Ces régressions traversent I'histoire du siècle finissant et ouvrent le nouveau sur une nouvelle et très vieille idée: présenter l'autre comme l'incarnation du mal et soi-même comme celle du bien. Ces figures, Fanon les décrit déjà dans son analyse de la situation coloniale dans Les Damnés de la terre: pour le colonisateur, le colonisé est l'incarnation du mal. Au-delà, il indique les effets dévastateurs, sur le plan subjectif, de cette configuration: celui désigné comme mal, figé 
sous le regard, éprouve d'abord de la honte désubjectivante, puis de la haine. Ce processus est aujourd hui d'une étrange actualité.

Aussi faut-il relire Les Damnés de la terre au-delà de la période historique circonscrite où fut écrit cet ouvrage, et à la lumière de [15] notre modernité. Que nous donne-t-elle à voir? La multiplication des laissés-pour-compte de la croissance, aussi bien au Sud qu'au Nord, mais aussi le renouvellement incessant de l'humiliation et de l'écrasement subjectif de tous ceux que cette même modernité désigne allégrement, face à la globalisation, comme les « sans »: sans patrie, sans territoire, mais aussi sans domicile, sans travail, sans papiers, sans droit à un espace de parole.

Lire ou relire Les Damnés de terre aide à comprendre ce qui se produit quand des êtres humains sont ainsi maintenus dans le registre de la privation: violences, recours aux régressions ethniques ou identitaires. Mais au-delà de ces thèmes insistants, l'actualité de Fanon réside aussi en ceci : de façon anticipatrice, à une époque où se renvoyaient dos à dos, d'un côté, l'analyse matérialiste de l'aliénation et des rapports de force et, de l'autre, une vision existentialiste ou culturaliste du sujet (ou même, sur le plan psychanalytique, une vision d'une aventure subjective coupée du monde environnant), il a tenté de mettre en place une nouvelle construction du savoir introduisant le corps, la langue et l'altérité comme expérience subjective nécessaire dans la construction même de l'avenir du politique. Cette démarche n'est au fond pas si éloignée de celle de l'école de Marcuse ou, plus encore, des préoccupations des psychanalystes politiques de Vienne qui furent laminés par la Seconde Guerre mondiale et leur exil forcé aux Etats-Unis.

Ce n'est donc pas un hasard si Fanon se présente comme d'une grande actualité. De par ses origines et son parcours, il recoupe les événements du siècle dernier dont il fut l'un des acteurs, aux prises avec les situations traumatiques qui ont scandé ce temps.

Il est actuel aussi par sa vie et le mouvement de sa pensée au-delà de ce que l'on nomme la faillite des idéologies, en cette époque de glo- 
balisation économique et d'exclusion du sujet, la phrase, écrite par Fanon jeune, et qui guide toute sa pensée en acte - «Oh mon corps, fais toujours de moi un homme qui interroge! »-, fait résonance chez beaucoup de jeunes de notre temps, quels que soient leur langue et leur lieu de naissance. 
[17]

\section{LES DAMNÉS DE LA TERRE \\ Préface à l'édition de 1961}

Par Jean-Paul Sartre

\section{Retour à la table des matières}

Il n'y a pas si longtemps, la terre comptait deux milliards d'habitants, soit cinq cents millions d'hommes et un milliard cinq cents millions d'indigènes. Les premiers disposaient du Verbe, les autres l'empruntaient. Entre ceux-là et ceux-ci, des roitelets vendus, des féodaux, une fausse bourgeoisie forgée de toutes pièces servaient d'intermédiaires. Aux colonies la vérité se montrait nue; les «métropoles » la préféraient vêtue; il fallait que l'indigène les aimât. Comme des mères, en quelque sorte. L'élite européenne entreprit de fabriquer un indigénat d'élite: on sélectionnait des adolescents, on leur marquait sur le front, au fer rouge, les principes de la culture occidentale, on leur fourrait dans la bouche des bâillons sonores, grands mots pâteux qui collaient aux dents; après un bref séjour en métropole, on les renvoyait chez eux, truqués. Ces mensonges vivants n'avaient plus rien à dire à leurs frères ; ils résonnaient; de Paris, de Londres, d'Amsterdam nous lancions des mots «Parthénon! Fraternité! » et, quelque part en Afrique, en Asie, des lèvres s'ouvraient : « ... thénon !... nité ! » C'était l'âge d'or. 
Il prit fin: les bouches s'ouvrirent seules; les voix jaunes et noires parlaient encore de notre humanisme mais c'était pour nous reprocher notre inhumanité. Nous écoutions sans déplaisir ces courtois exposés d'amertume. D'abord ce fut un émerveillement fier : comment? Ils causent tout seuls? Voyez pourtant ce que nous avons fait d'eux! Nous ne doutions pas qu'ils acceptassent notre idéal puisqu'ils nous accusaient de n'y être pas fidèles; pour le coup, l'Europe crut à sa mission: elle avait hellénisé les Asiatiques, créé cette espèce nouvelle, les nègres gréco-latins. Nous ajoutions, tout à fait entre nous, pratiques: et puis laissons-les gueuler, ça les soulage; chien qui aboie ne mord pas.

[18]

Une autre génération vint, qui déplaça la question. Ses écrivains, ses poètes, avec une incroyable patience, essayèrent de nous expliquer que nos valeurs collaient mal avec la vérité de leur vie, qu'ils ne pouvaient ni tout à fait les rejeter ni les assimiler. En gros, cela voulait dire : vous faites de nous des monstres, votre humanisme nous prétend universels et vos pratiques racistes nous particularisent. Nous les écoutions, très décontractés: les administrateurs coloniaux ne sont pas payés pour lire Hegel, aussi bien le lisent-ils peu, mais ils n'ont pas besoin de ce philosophe pour savoir que les consciences malheureuses s'empêtrent dans leurs contradictions. Efficacité nulle. Donc perpétuons leur malheur, il n'en sortira que du vent. S'il y avait, nous disaient les experts, l'ombre d'une revendication dans leurs gémissements, ce serait celle de l'intégration. Pas question de l'accorder, bien entendu : on eût ruiné le système qui repose, comme vous savez, sur la surexploitation. Mais il suffirait de tenir devant leurs yeux cette carotte : ils galoperaient. Quant à se révolter, nous étions bien tranquilles: quel indigène conscient s'en irait massacrer les beaux fils de l'Europe à seule fin de devenir européen comme eux? Bref, nous encouragions ces mélancolies et ne trouvâmes pas mauvais, une fois, de décerner le prix Goncourt à un nègre : c'était avant 39.

1961. Écoutez: «Ne perdons pas de temps en stériles litanies ou en mimétismes nauséabonds. Quittons cette Europe qui n'en finit pas 
de parler de l'homme tout en le massacrant partout où elle le rencontre, à tous les coins de ses propres rues, à tous les coins du monde. Voici des siècles... qu'au nom d'une prétendue "aventure spirituelle" elle étouffe la quasi-totalité de l'humanité. »Ce ton est neuf. Qui ose le prendre? Un Africain, homme du tiers monde, ancien colonisé. Il ajoute: «L'Europe a acquis une telle vitesse folle, désordonnée... qu'elle va vers des abîmes dont il vaut mieux s'éloigner. » Autrement dit : elle est foutue. Une vérité qui n'est pas bonne à dire mais dont n'est-ce pas, mes chers co-continentaux? - nous sommes tous, entre chair et cuir, convaincus.

[19]

Il faut faire une réserve, pourtant. Quand un Français, par exemple, dit à d'autres Français: « Nous sommes foutus!»-ce qui, à ma connaissance, se produit à peu près tous les jours depuis $1930-$, c'est un discours passionnel, brûlant de rage et d'amour, l'orateur se met dans le bain avec tous ses compatriotes. Et puis il ajoute généralement : « À moins que... » On voit ce que c'est : il n'y a plus une faute à commettre; si ses recommandations ne sont pas suivies à la lettre, alors et seulement alors le pays se désintégrera. Bref, c'est une menace suivie d'un conseil et ces propos choquent d'autant moins qu'ils jaillissent de l'intersubjectivité nationale. Quand Fanon, au contraire, dit de l'Europe qu'elle court à sa perte, loin de pousser un cri d'alarme, il propose un diagnostic. Ce médecin ne prétend ni la condamner sans recours - on a vu des miracles - ni lui donner les moyens de guérir : il constate qu'elle agonise. Du dehors, en se basant sur les symptômes qu'il a pu recueillir. Quant à la soigner, non : il a d'autres soucis en tête: qu'elle crève ou qu'elle survive, il s'en moque. Par cette raison, son livre est scandaleux. Et si vous murmurez, rigolards et gênés: «Qu'est-ce qu'il nous met! », la vraie nature du scandale vous échappe : car Fanon ne vous « met» rien du tout ; son ouvrage - si brûlant pour d'autres - reste pour vous glacé ; on y parle de vous souvent, à vous jamais. Finis les Goncourt noirs et les Nobel jaunes : il ne reviendra plus le temps des lauréats colonisés. Un ex-indigène « de langue française " plie cette langue à des exigences nouvelles, en use et 
s'adresse aux seuls colonisés: "Indigènes de tous les pays sousdéveloppés, unissez-vous! 》Quelle déchéance: pour les pères, nous étions les uniques interlocuteurs; les fils ne nous tiennent même plus pour des interlocuteurs valables: nous sommes les objets du discours. Bien sûr, Fanon mentionne au passage nos crimes fameux, Sétif, Hanoï, Madagascar, mais il ne perd pas sa peine à les condamner : il les utilise. $S$ 'il démonte les tactiques du colonialisme, le jeu complexe des relations qui unissent et qui opposent les colons aux «métropolitains » c'est pour ses frères; son but est de leur apprendre à nous déjouer.

[20]

Bref, le tiers monde se découvre et se parle par cette voix. On sait qu'il n'est pas homogène et qu' on y trouve encore des peuples asservis, d'autres qui ont acquis une fausse indépendance, d'autres qui se battent pour conquérir la souveraineté, d'autres enfin qui ont gagné la liberté plénière mais qui vivent sous la menace constante d'une agression impérialiste. Ces différences sont nées de l'histoire coloniale, cela veut dire de l'oppression. Ici la Métropole s'est contentée de payer quelques féodaux: là, divisant pour régner, elle a fabriqué de toutes pièces une bourgeoisie de colonisés: ailleurs elle a fait coup double: la colonie est à la fois d'exploitation et de peuplement. Ainsi l'Europe a-t-elle multiplié les divisions, les oppositions, forgé des classes et parfois des racismes, tenté par tous les expédients de provoquer et d'accroître la stratification des sociétés colonisées. Fanon ne dissimule rien: pour lutter contre nous, l'ancienne colonie doit lutter contre elle-même. Ou plutôt les deux ne font qu'un. Au feu du combat, toutes les barrières intérieures doivent fondre, l'impuissante bourgeoisie d'affairistes et de compradores, le prolétariat urbain, toujours privilégié, le lumpenproletariat des bidonvilles, tous doivent s'aligner sur les positions des masses rurales, véritable réservoir de l'armée nationale et révolutionnaire; dans ces contrées dont le colonialisme a délibérément stoppé le développement, la paysannerie, quand elle se révolte, apparaît très vite comme la classe radicale : elle connaît l'oppression nue, elle en souffre beaucoup plus que les travailleurs des villes et, pour l'empêcher de mourir de faim, il ne faut rien 
de moins qu'un éclatement de toutes les structures. Qu'elle triomphe, la Révolution nationale sera socialiste: qu'on arrête son élan, que la bourgeoisie colonisée prenne le pouvoir, le nouvel État, en dépit d'une souveraineté formelle, reste aux mains des impérialistes. C'est ce qu'illustre assez bien l'exemple du Katanga. Ainsi l'unité du tiers monde n'est pas faite: c'est une entreprise en cours qui passe par l'union, en chaque pays, après comme avant l'indépendance, de tous les colonisés sous le commandement de la classe paysanne. Voilà ce que Fanon explique à ses frères d'Afrique, d'Asie, [21] d'Amérique latine: nous réaliserons tous ensemble et partout le socialisme révolutionnaire ou nous serons battus un à un par nos anciens tyrans. Il ne dissimule rien : ni les faiblesses, ni les discordes, ni les mystifications. Ici le mouvement prend un mauvais départ; là, après de foudroyants succès, il est en perte de vitesse ; ailleurs il s'est arrêté : si l'on veut qu'il reprenne, il faut que les paysans jettent leur bourgeoisie à la mer. Le lecteur est sévèrement mis en garde contre les aliénations les plus dangereuses: le leader, le culte de la personne, la culture occidentale et, tout aussi bien, le retour du lointain passé de la culture africaine : la vraie culture c'est la Révolution: cela veut dire qu'elle se forge à chaud. Fanon parle à voix haute: nous, les Européens, nous pouvons l'entendre: la preuve en est que vous tenez ce livre entre vos mains: ne craint-il pas que les puissances coloniales tirent profit de sa sincérité?

Non. Il ne craint rien. Nos procédés sont périmés: ils peuvent retarder parfois l'émancipation, ils ne l'arrêteront pas. Et n'imaginons pas que nous pourrons rajuster nos méthodes: le néo-colonialisme, ce rêve paresseux des Métropoles, c'est du vent; les «troisièmes forces » n'existent pas ou bien ce sont les bourgeoisies bidon que le colonialisme a déjà mises au pouvoir. Notre machiavélisme a peu de prises sur ce monde fort éveillé qui a dépisté l'un après l'autre nos mensonges. Le colon n'a qu'un recours : la force, quand il lui en teste; l'indigène n'a qu'un choix: la servitude ou la souveraineté. Qu'est-ce que ça peut lui faire, à Fanon, que vous lisiez ou non son ouvrage, c'est à ses frères qu'il dénonce nos vieilles malices, sûr que nous n'en avons 
pas de rechange. C'est à eux qu'il dit: l'Europe a mis les pattes sur nos continents, il faut les taillader jusqu'à ce qu'elle les retire; le moment nous favorise : rien n'arrive à Bizerte, à Élisabethville, dans le bled algérien que la terre entière n'en soit informée; les blocs prennent des partis contraires, ils se tiennent en respect, profitons de cette paralysie, entrons dans l'histoire et que notre irruption la rende universelle pour la première fois: battons-nous: à défaut d'autres armes, la patience du couteau suffira.

[22]

Européens, ouvrez ce livre, entrez-y. Après quelques pas dans la nuit vous verrez des étrangers réunis autour d'un feu, approchez, écoutez: ils discutent du sort qu'ils réservent à vos comptoirs, aux mercenaires qui les défendent. Ils vous verront peut-être, mais ils continueront de parler entre eux, sans même baisser la voix. Cette indifférence frappe au cœur: les pères, créatures de l'ombre, vos créatures, c'étaient des âmes mortes, vous leur dispensiez la lumière, ils ne s'adressaient qu'à vous, et vous ne preniez pas la peine de répondre à ces zombies. Les fils vous ignorent : un feu les éclaire et les réchauffe, qui n'est pas le vôtre. Vous, à distance respectueuse, vous vous sentirez furtifs, nocturnes, transis: chacun son tour; dans ces ténèbres d'où va surgir une autre aurore, les zombies, c'est vous.

En ce cas, direz-vous, jetons cet ouvrage par la fenêtre. Pourquoi le lire puisqu'il n'est pas écrit pour nous? Pour deux motifs dont le premier est que Fanon vous explique à ses frères et démonte pour eux le mécanisme de nos aliénations : profitez-en pour vous découvrir à vousmêmes dans votre vérité d'objets. Nos victimes nous connaissent par leurs blessures et par leurs fers: c'est ce qui rend leur témoignage irréfutable. Il suffît qu'elles nous montrent ce que nous avons fait d'elles pour que nous connaissions ce que nous avons fait de nous. Estce utile? Oui, puisque l'Europe est en grand danger de crever. Mais, direz-vous encore, nous vivons dans la Métropole et nous réprouvons les excès. Il est vrai : vous n'êtes pas des colons, mais vous ne valez pas mieux. Ce sont vos pionniers, vous les avez envoyés, outre-mer, ils vous ont enrichis; vous les aviez prévenus: s'ils faisaient couler trop 
de sang, vous les désavoueriez du bout des lèvres; de la même manière un État - quel qu'il soit - entretint à l'étranger une tourbe d'agitateurs, de provocateurs et d'espions qu'il désavoue quand on les prend. Vous, si libéraux, si humains, qui poussez l'amour de la culture jusqu'à la préciosité, vous faites semblant d'oublier que vous avez des colonies et qu'on y massacre en votre nom. Fanon révèle à ses camarades - à certains d'entre eux, surtout, qui demeurent un peu trop occidentalisés - la solidarité des «métropolitains » et [23] de leurs agents coloniaux. Ayez le courage de le lire: par cette première raison qu'il vous fera honte et que la honte, comme a dit Marx, est un sentiment révolutionnaire. Vous voyez: moi aussi je ne peux me déprendre de l'illusion subjective. Moi aussi, je vous dis: « Tout est perdu, à moins que... » Européen, je vole le livre d'un ennemi et j'en fais un moyen de guérir l'Europe. Profitez-en.

*

Et voici la seconde raison: si vous écartez les bavardages fascistes de Sorel, vous trouverez que Fanon est le premier depuis Engels à remettre en lumière l'accoucheuse de l'histoire. Et n'allez pas croire qu'un sang trop vif ou que des malheurs d'enfance lui aient donné pour la violence je ne sais quel goût singulier : il se fait l'interprète de la situation, rien de plus. Mais cela suffit pour qu'il constitue, étape par étape, la dialectique que l'hypocrisie libérale vous cache et qui nous a produits tout autant que lui.

Au siècle dernier, la bourgeoisie tient les ouvriers pour des envieux, déréglés par de grossiers appétits, mais elle prend soin d'inclure ces grands brutaux dans notre espèce : à moins d'être hommes et libres, comment pourraient-ils vendre librement leur force de travail. En France, en Angleterre, I'humanisme se prétend universel.

Avec le travail forcé, c'est tout le contraire: pas de contrat; en plus de ça, il faut intimider: donc l'oppression se montre. Nos soldats, outre-mer, repoussant l'universalisme métropolitain, appliquent au genre humain le numerus clausus: puisque nul ne peut sans crime dépouiller son semblable, l'asservir ou le tuer, ils posent en principe que 
le colonisé n'est pas le semblable de l'homme. Notre force de frappe a reçu mission de changer cette abstraite certitude en réalité : ordre est donné de ravaler les habitants du territoire annexé au niveau du singe supérieur pour justifier le colon de les traiter en bêtes de somme. La violence coloniale ne se donne pas seulement le but de tenir en respect ces hommes asservis, elle cherche à les déshumaniser. Rien ne [24] sera ménagé pour liquider leurs traditions, pour substituer nos langues aux leurs, pour détruire leur culture sans leur donner la nôtre: on les abrutira de fatigue. Dénourris, malades, s'ils résistent encore la peur terminera le job: on braque sur le paysan des fusils; viennent des civils qui s'installent sur sa terre et le contraignent par la cravache à la cultiver pour eux. S'il résiste, les soldats tirent, c'est un homme mort ; s'il cède, il se dégrade, ce n'est plus un homme; la honte et la crainte vont fissurer son caractère, désintégrer sa personne. L'affaire est menée tambour battant, par des experts: ce n'est pas d'aujourd'hui que datent les «services psychologiques». Ni le lavage de cerveau. Et pourtant, malgré tant d'efforts, le but n'est atteint nulle part: au Congo, où l'on coupait les mains des nègres, pas plus qu'en Angola où, tout récemment, on trouait les lèvres des mécontents pour les fermer par des cadenas. Et je ne prétends pas qu'il soit impossible de changer un homme en bête: je dis qu'on n'y parvient pas sans l'affaiblir considérablement; les coups ne suffisent jamais, il faut forcer sur la dénutrition. C'est l'ennui, avec la servitude: quand on domestique un membre de notre espèce, on diminue son rendement et, si peu qu'on lui donne, un homme de basse-cour finit par coûter plus qu'il ne rapporte. Par cette raison les colons sont obligés d'arrêter le dressage à la mi-temps: le résultat, ni homme ni bête, c'est l'indigène. Battu, sous-alimenté, malade, apeuré, mais jusqu'à un certain point seulement, il $a$, jaune, noir ou blanc, toujours les mêmes traits de caractère: c'est un paresseux, sournois et voleur, qui vit de rien et ne connaît que la force.

Pauvre colon: voilà sa contradiction mise à nu. Il devrait, comme fait, dit-on, le génie, tuer ceux qu'il pille. Or cela n'est pas possible: ne faut-il pas aussi qu'il les exploite? Faute de pousser le massacre 
jusqu'au génocide, et la servitude jusqu'à l'abêtissement, il perd les pédales, l'opération se renverse, une implacable logique la mènera jusqu'à la décolonisation.

Pas tout de suite. D'abord l'Européen règne: il a déjà perdu mais ne s'en aperçoit pas; il ne sait pas encore que les indigènes sont de faux indigènes : il leur fait du mal, à l'entendre, pour [25] détruire ou pour refouler le mal qu'ils ont en eux; au bout de trois générations, leurs pernicieux instincts ne renaîtront plus. Quels instincts? Ceux qui poussent les esclaves à massacrer le maître? Comment n'y reconnaît-il pas sa propre cruauté retournée contre lui? La sauvagerie de ces paysans opprimés, comment n'y retrouve-t-il pas sa sauvagerie de colon qu'ils ont absorbée par tous les pores et dont ils ne se guérissent pas? La raison est simple: ce personnage impérieux, affolé par sa toute-puissance et par la peur de la perdre, ne se rappelle plus très bien qu'il a été un homme: il se prend pour une cravache ou pour un fusil ; il en est venu à croire que la domestication des « races inférieures» s'obtient par le conditionnement de leurs réflexes. Il néglige la mémoire humaine, les souvenirs ineffaçables; et puis, surtout, il y a ceci qu'il n'a peut-être jamais su : nous ne devenons ce que nous sommes que par la négation intime et radicale de ce qu'on a fait de nous. Trois générations? Dès la seconde, à peine ouvraient-ils les yeux, les fils ont vu battre leurs pères. En termes de psychiatrie, les voilà "traumatisés». Pour la vie. Mais ces agressions sans cesse renouvelées, loin de les porter à se soumettre, les jettent dans une contradiction insupportable dont l'Européen, tôt ou tard, fera les frais. Après cela, qu'on les dresse à leur tour, qu' on leur apprenne la honte, la douleur et la faim: on ne suscitera dans leurs corps qu'une rage volcanique dont la puissance est égale à celle de la pression qui s'exerce sur eux. Ils ne connaissent, disiez-vous, que la force? Bien sûr; d'abord ce ne sera que celle du colon et, bientôt, que la leur, cela veut dire : la même rejaillissant sur nous comme notre reflet vient du fond d'un miroir à notre rencontre. Ne vous y trompez pas : par cette folle rogne, par cette bile et ce fiel, par leur désir permanent de nous tuer, par la contracture permanente de muscles puissants qui ont peur de se dé- 
nouer, ils sont hommes: par le colon, qui les veut hommes de peine, et contre lui. Aveugle encore, abstraite, la haine est leur seul trésor: le Maître la provoque parce qu'il cherche à les abêtir, il échoue à la briser parce que ses intérêts l'arrêtent à mi-chemin; ainsi les faux indigènes sont humains encore, par la puissance et [26] l'impuissance de l'oppresseur qui se transforment, chez eux, en un refus entêté de la condition animale. Pour le reste on a compris; ils sont paresseux, bien sûr : c'est du sabotage. Sournois, voleurs: parbleu; leurs menus larcins marquent le commencement d'une résistance encore inorganisée. Cela ne suffit pas : il en est qui s'affirment en se jetant à mains nues contre les fusils: ce sont leurs héros; et d'autres se font hommes en assassinant des Européens. On les abat: brigands et martyrs, leur supplice exalte les masses terrifiées.

Terrifiées, oui : en ce nouveau moment, l'agression coloniale s'intériorise en Terreur chez les colonisés. Par là je n'entends pas seulement la crainte qu'ils éprouvent devant nos inépuisables moyens de répression mais aussi celle que leur inspire leur propre fureur. Ils sont coincés entre nos armes qui les visent et ces effrayantes pulsions, ces désirs de meurtre qui montent du fond des cours et qu'ils ne reconnaissent pas toujours : car ce n'est pas d'abord leur violence, c'est la nôtre, retournée, qui grandit et les déchire; et le premier mouvement de ces opprimés est d'enfouir profondément cette inavouable colère que leur morale et la nôtre réprouvent et qui n'est pourtant que le dernier réduit de leur humanité. Lisez Fanon: vous saurez que, dans le temps de leur impuissance, la folie meurtrière est l'inconscient collectif des colonisés.

Cette furie contenue, faute d'éclater, tourne en rond et ravage les opprimés eux-mêmes. Pour s'en libérer, ils en viennent à se massacrer entre eux: les tribus se battent les unes contre les autres faute de pouvoir affronter l'ennemi véritable - et vous pouvez compter sur la politique coloniale pour entretenir leurs rivalités; le frère, levant le couteau contre son frère, croit détruire, une fois pour toutes, l'image détestée de leur avilissement commun. Mais ces victimes expiatoires n'apaisent pas leur soif de sang; ils ne s'empêcheront de marcher 
contre les mitrailleuses qu'en se faisant nos complices: cette déshumanisation qu'ils repoussent, ils vont de leur propre chef en accélérer les progrès. Sous les yeux amusés du colon, ils se prémuniront contre eux-mêmes par des barrières surnaturelles, [27] tantôt ranimant de vieux mythes terribles, tantôt se ligotant par des rites méticuleux: ainsi l'obsédé fuit son exigence profonde en s'infligeant des manies qui le requièrent à chaque instant. Ils dansent : ça les occupe : ça dénoue leurs muscles douloureusement contractés et puis la danse mime en secret, souvent à leur insu, le Non qu'ils ne peuvent dire, les meurtres qu'ils n'osent commettre. En certaines régions ils usent de ce dernier recours : la possession. Ce qui était autrefois le fait religieux dans sa simplicité, une certaine communication du fidèle avec le sacré, ils en font une arme contre le désespoir et l'humiliation: les zars, les loas, les Saints de la Sainterie descendent en eux, gouvernent leur violence et la gaspillent en transes jusqu'à l'épuisement. En même temps ces hauts personnages les protègent : cela veut dire que les colonisés se défendent de l'aliénation coloniale en renchérissant sur l'aliénation religieuse. Avec cet unique résultat, au bout du compte, qu'ils cumulent les deux aliénations et que chacune se renforce par l'autre. Ainsi, dans certaines psychoses, las d'être insultés tous les jours, les hallucinés s'avisent un beau matin d'entendre une voix d'ange qui les complimente : les quolibets ne cessent pas pour autant : désormais ils alternent avec la félicitation. C'est une défense et c'est la fin de leur aventure: la personne est dissociée, le malade s'achemine vers la démence. Ajoutez, pour quelques malheureux rigoureusement sélectionnés, cette autre possession dont j'ai parlé plus haut: la culture occidentale. À leur place, direz-vous, j'aimerais encore mieux mes zars que l'Acropole. Bon : vous avez compris. Pas tout à fait cependant car vous n'êtes pas à leur place. Pas encore. Sinon vous sauriez qu'ils ne peuvent pas choisir: ils cumulent. Deux mondes, ça fait deux possessions : on danse toute la nuit, à l'aube on se presse dans les églises pour entendre la messe; de jour en jour la fêlure s'accroît. Notre ennemi trahit ses frères et se fait notre complice; ses frères en font autant. L'indigénat est une névrose introduite et maintenue par le colon chez les colonisés avec leur consentement. 
Réclamer et renier, tout à la fois, la condition humaine: la contradiction est explosive. Aussi bien explose-t-elle, vous le [28] savez comme moi. Et nous vivons au temps de la déflagration : que la montée des naissances accroisse la disette, que les nouveaux venus aient à redouter de vivre un peu plus que de mourir, le torrent de la violence emporte toutes les barrières. En Algérie, en Angola, on massacre à vue les Européens. C'est le moment du boomerang, le troisième temps de la violence: elle revient sur nous, elle nous frappe et, pas plus que les autres fois, nous ne comprenons que c'est le nôtre. Les «libéraux » restent hébétés: ils reconnaissent que nous n'étions pas assez polis avec les indigènes, qu'il eût été plus juste et plus prudent de leur accorder certains droits dans la mesure du possible; ils ne demandaient pas mieux que de les admettre par fournées et sans parrain dans ce club si fermé, notre espèce : et voici que ce déchaînement barbare et fou ne les épargne pas plus que les mauvais colons. La gauche métropolitaine est gênée: elle connaît le véritable sort des indigènes, l'oppression sans merci dont ils font l'objet, elle ne condamne pas leur révolte, sachant que nous avons tout fait pour la provoquer. Mais tout de même, pense-t-elle, il y a des limites: ces guérilleros devraient tenir à couur de se montrer chevaleresques; ce serait le meilleur moyen de prouver qu'ils sont des hommes. Parfois elle les gourmande : « Vous allez trop fort, nous ne vous soutiendrons plus. "Ils s'en foutent: pour ce que vaut le soutien qu'elle leur accorde, elle peut tout aussi bien se le mettre au cul. Dès que leur guerre a commencé, ils ont aperçu cette vérité rigoureuse: nous nous valons tous tant que nous sommes, nous avons tous profité d'eux, ils n'ont rien à prouver, ils ne feront de traitement de faveur à personne. Un seul devoir, un seul objectif : chasser le colonialisme par tous les moyens. Et les plus avisés d'entre nous seraient, à la rigueur, prêts à l'admettre mais ils ne peuvent s'empêcher de voir dans cette épreuve de force le moyen tout inhumain que des sous-hommes ont pris pour se faire octroyer une charte d'humanité : qu'on l'accorde au plus vite et qu'ils tâchent alors, par des entreprises pacifiques, de la mériter. Nos belles âmes sont racistes. 
Elles auront profit à lire Fanon; cette violence irrépressible, il le montre parfaitement, n'est pas une absurde tempête ni la [29] résurrection d'instincts sauvages ni même un effet du ressentiment : c'est I'homme lui-même se recomposant. Cette vérité, nous l'avons sue, je crois, et nous l'avons oubliée : les marques de la violence, nulle douceur ne les effacera: c'est la violence qui peut seule les détruire. Et le colonisé se guérit de la névrose coloniale en chassant le colon par les armes. Quand sa rage éclate, il retrouve sa transparence perdue, il se connaît dans la mesure même où il se fait ; de loin nous tenons sa guerre comme le triomphe de la barbarie: mais elle procède par elle-même à l'émancipation progressive du combattant, elle liquide en lui et hors de lui, progressivement, les ténèbres coloniales. Dès qu'elle commence, elle est sans merci. Il faut rester terrifié ou devenir terrible; cela veut dire: s'abandonner aux dissociations d'une vie truquée ou conquérir l'unité natale. Quand les paysans touchent des fusils, les vieux mythes pâlissent, les interdits sont un à un renversés: l'arme d'un combattant, c'est son humanité. Car, en le premier temps de la révolte, il faut tuer: abattre un Européen c'est faire d'une pierre deux coups, supprimer en même temps un oppresseur et un opprimé : restent un homme mort et un homme libre; le survivant, pour la première fois, sent un sol national sous la plante de ses pieds. Dans cet instant la Nation ne s'éloigne pas de lui : on la trouve où il va, où il est - jamais plus loin, elle se confond avec sa liberté. Mais, après la première surprise, l'armée coloniale réagit : il faut s' unir ou se faire massacrer. Les discordes tribales s'atténuent, tendent à disparaître: d'abord parce qu'elles mettent en danger la Révolution, et plus profondément parce qu'elles n'avaient d'autre office que de dériver la violence vers de faux ennemis. Quand elles demeurent - comme au Congo -, c'est qu'elles sont entretenues par les agents du colonialisme. La Nation se met en marche: pour chaque frère elle est partout où d'autres frères combattent. Leur amour fraternel est l'envers de la haine qu'ils vous portent : frères en ceci que chacun d'eux a tué, peut, d'un instant à l'autre, avoir tué. Fanon montre à ses lecteurs les limites de la «spontanéité », la nécessité et les dangers de "l'organisation». Mais, quelle que soit l'immensité de la tâche, à [30] chaque dé- 
veloppement de l'entreprise la conscience révolutionnaire s'approfondit. Les derniers complexes s'envolent : qu'on vienne un peu nous parler du « complexe de dépendance » chez le soldat de l'ALN. Libéré de ses œillères, le paysan prend connaissance de ses besoins: ils le tuaient mais il tentait de les ignorer; il les découvre comme des exigences infinies. En cette violence populaire - pour tenir cinq ans, huit ans comme ont fait les Algériens, les nécessités militaires, sociales et politiques ne se peuvent distinguer. La guerre - ne fût-ce qu'en posant la question du commandement et des responsabilités - institue de nouvelles structures qui seront les premières institutions de la paix. Voici donc I'homme instauré jusque dans des traditions nouvelles, filles futures d'un horrible présent, le voici légitimé par un droit qui va naître, qui naît chaque jour au feu : avec le dernier colon tué, rembarqué ou assimilé, l'espèce minoritaire disparaît, cédant la place à la fraternité socialiste. Et ce n'est pas encore assez: ce combattant brûle les étapes; vous pensez bien qu'il ne risque pas sa peau pour se retrouver au niveau du vieil homme «métropolitain». Voyez sa patience : peut-être rêve-t-il quelquefois d'un nouveau Dien-Bien-Phu; mais croyez qu'il n'y compte pas vraiment : c'est un gueux luttant, dans sa misère, contre des riches puissamment armés. En attendant les victoires décisives et, souvent, sans rien attendre, il travaille ses adversaires à l'écœurement. Cela n'ira pas sans d'effroyables pertes: I'armée coloniale devient féroce: quadrillages, ratissages, regroupements, expéditions punitives ; on massacre les femmes et les enfants. Il le sait : cet homme neuf commence sa vie d'homme par la fin; il se tient pour un mort en puissance. Il sera tué : ce n'est pas seulement qu'il en accepte le risque, c'est qu'il en a la certitude; ce mort en puissance a perdu sa femme, ses fils: il a vu tant d'agonies qu'il veut vaincre plutôt que survivre: d'autres profiteront de la victoire, pas lui : il est trop las. Mais cette fatigue du cœur est à l'origine d'un incroyable courage. Nous trouvons notre humanité en deçà de la mort et du désespoir, il la trouve au-delà des supplices et de la mort. Nous avons été les semeurs de vent ; la tempête, c'est lui. Fils de la violence, [31] il puise en elle à chaque instant son humanité : nous étions hommes à ses dépens, il se fait homme aux nôtres. Un autre homme: de meilleure qualité. 
Ici Fanon s'arrête. Il a montré la route: porte-parole des combattants, il a réclamé I'union, l'unité du continent africain contre toutes les discordes et tous les particularismes. Son but est atteint. S'il voulait décrire intégralement le fait historique de la décolonisation, il lui faudrait parler de nous: ce qui n'est certes pas son propos. Mais, quand nous avons fermé le livre, il se poursuit en nous, malgré son auteur: car nous éprouvons la force des peuples en révolution et nous y répondons par la force. Il y a donc un nouveau moment de la violence et c'est à nous, cette fois, qu'il faut revenir car elle est en train de nous changer dans la mesure où le faux indigène se change à travers elle. $\grave{A}$ chacun de mener ses réflexions comme il veut. Pourvu toutefois qu'il réfléchisse: dans l'Europe d'aujourd'hui, tout étourdie par les coups qu' on lui porte, en France, en Belgique, en Angleterre, le moindre divertissement de la pensée est une complicité criminelle avec le colonialisme. Ce livre n'avait nul besoin d'une préface. D'autant moins qu'il ne $s$ 'adresse pas à nous. J'en ai fait une, cependant, pour mener jusqu'au bout la dialectique: nous aussi, gens de l'Europe, on nous décolonise: cela veut dire qu'on extirpe par une opération sanglante le colon qui est en chacun de nous. Regardons-nous, si nous en avons le courage, et voyons ce qu'il advient de nous.

Il faut affronter d'abord ce spectacle inattendu : le strip-tease de notre humanisme. Le voici tout nu, pas beau : ce n'était qu'une idéologie menteuse, l'exquise justification du pillage: ses tendresses et sa préciosité cautionnaient nos agressions. Ils ont bonne mine, les nonviolents : ni victimes ni bourreaux! Allons! Si vous n'êtes pas victimes, quand le gouvernement que vous avez plébiscité, quand l'année où vos jeunes frères ont servi, sans hésitation ni remords, ont entrepris un "génocide», vous êtes indubitablement des bourreaux. Et si vous choisissez [32] d'être victimes, de risquer un jour ou deux de prison, vous choisissez simplement de tirer votre épingle du jeu. Vous ne l'en tirerez pas : il faut qu'elle y reste jusqu'au bout. Comprenez enfin ce$\mathrm{ci}$ : si la violence a commencé ce soir, si l'exploitation ni l'oppression n'ont jamais existé sur terre, peut-être la non-violence affichée peut 
apaiser la querelle. Mais si le régime tout entier et jusqu'à vos nonviolentes pensées sont conditionnés par une oppression millénaire, votre passivité ne sert qu'à vous ranger du côté des oppresseurs.

Vous savez bien que nous sommes des exploiteurs. Vous savez bien que nous avons pris l'or et les métaux puis le pétrole des « continents neufs » et que nous les avons ramenés dans les vieilles métropoles. Non sans d'excellents résultats: des palais, des cathédrales, des capitales industrielles; et puis quand la crise menaçait, les marchés coloniaux étaient là pour l'amortir ou la détourner. L'Europe, gavée de richesses, accorda de jure l'humanité à tous ses habitants: un homme, chez nous, ça veut dire un complice puisque nous avons tous profité de l'exploitation coloniale. Ce continent gras et blême finit par donner dans ce que Fanon nomme justement le «narcissisme ». Cocteau s'agaçait de Paris, «cette ville qui parle tout le temps d'elle-même». Et l'Europe, que fait-elle d'autre? Et ce monstre sureuropéen, I'Amérique du Nord? Quel bavardage: liberté, égalité, fraternité, amour, honneur, patrie, que sais-je? Cela ne nous empêchait pas de tenir en même temps des discours racistes, sale nègre, sale juif, sale raton. De bons esprits, libéraux et tendres - des néo-colonialistes, en somme - se prétendaient choqués par cette inconséquence ; erreur ou mauvaise foi : rien de plus conséquent, chez nous, qu' un humanisme raciste puisque l'Européen n'a pu se faire homme qu'en fabriquant des esclaves et des monstres. Tant qu'il y eut un indigénat, cette imposture ne fut pas démasquée: on trouvait dans le genre humain une abstraite postulation d'universalité qui servait à couvrir des pratiques plus réalistes: il y avait, de l'autre côté des mers, une race de sous-hommes qui, grâce à nous, dans mille ans peut-être, accéderait à notre état. Bref on confondait le [33] genre avec l'élite. Aujourd'hui l'indigène révèle sa vérité : du coup, notre club si fermé révèle sa faiblesse: ce n'était ni plus ni moins qu'une minorité. Il y a pis: puisque les autres se font hommes contre nous, il apparaît que nous sommes les ennemis du genre humain: l'élite révèle sa vraie nature: un gang. Nos chères valeurs perdent leurs ailes; à les regarder de près, on n'en trouvera pas une qui ne soit tachée de sang. S'il vous faut un exemple, rappelez-vous 
ces grands mots: que c'est généreux, la France. Généreux, nous? Et Sétif? Et ces huit années de guerre féroce qui ont coûté la vie à plus d'un million d'Algériens? Et la gégène. Mais comprenez bien qu'on ne nous reproche pas d'avoir trahi je ne sais quelle mission : pour la bonne raison que nous n'en avions aucune. C'est la générosité même qui est en cause ; ce beau mot chantant n'a qu'un sens : statut octroyé. Pour les hommes d'en face, neufs et délivrés, personne n'a le pouvoir ni le privilège de rien donner à personne. Chacun a tous les droits. Sur tous: et notre espèce, lorsqu'un jour elle se sera faite, ne se définira pas comme la somme des habitants du globe mais comme l'unité infinie de leurs réciprocités. Je m'arrête; vous finirez le travail sans peine; il suffit de regarder en face, pour la première et pour la dernière fois, nos aristocratiques vertus : elles crèvent: comment survivraient-elles à l'aristocratie de sous-hommes qui les a engendrées. Il y a quelques années, un commentateur bourgeois - et colonialiste - pour défendre I'Occident n'a trouvé que ceci : « Nous ne sommes pas des anges. Mais nous, du moins, nous avons des remords. » Quel aveu! Autrefois notre continent avait d'autres flotteurs: le Parthénon, Chartres, les Droits de l'homme, la svastika. On sait à présent ce qu'ils valent: et l'on ne prétend plus nous sauver du naufrage que par le sentiment très chrétien de notre culpabilité. C'est la fin, comme vous voyez: I'Europe fait eau de toute part. Que s'est-il donc passé ? Ceci, tout simplement, que nous étions les sujets de l'histoire et que nous en sommes à présent les objets. Le rapport des forces s'est renversé, la décolonisation est en cours: tout ce que nos mercenaires peuvent tenter c'est d'en retarder l'achèvement.

[34]

Encore faut-il que les vieilles «métropoles»y mettent le paquet, qu'elles engagent dans une bataille d'avance perdue toutes leurs forces. Cette vieille brutalité coloniale qui a fait la gloire douteuse des Bugeaud, nous la retrouvons, à la fin de l'aventure, décuplée, insuffisante. On envoie le contingent en Algérie, il s'y maintient depuis sept ans sans résultat. La violence a changé de sens; victorieux nous l'exercions sans qu'elle parût nous altérer: elle décomposait les au- 
tres et nous, les hommes, notre humanisme restait intact; unis par le profit les métropolitains baptisaient fraternité, amour, la communauté de leurs crimes: aujourd'hui la même, partout bloquée, revient sur nous à travers nos soldats, s'intériorise et nous possède. L'involution commence : le colonisé se recompose et nous, ultras et libéraux, colons et «métropolitains», nous nous décomposons. Déjà la rage et la peur sont nues: elles se montrent à découvert dans les « ratonnades» d'Alger. Où sont les sauvages, à présent? Où est la barbarie? Rien ne manque, pas même le tam-tam: les klaxons rythment «Algérie française » pendant que les Européens font brûler vifs des Musulmans. Il n'y a pas si longtemps, Fanon le rappelle, des psychiatres en congrès s'affligeaient de la criminalité indigène: ces gens-là s'entre-tuent, disaient-ils, cela n'est pas normal; le cortex de l'Algérien doit être sous-développé. En Afrique centrale d'autres ont établi que "I'Africain utilise très peu ses lobes frontaux». Ces savants auraient intérêt aujourd'hui à poursuivre leur enquête en Europe et particulièrement chez les Français. Car nous aussi, depuis quelques années, nous devons être atteints de paresse frontale: les patriotes assassinent un peu leurs compatriotes; en cas d'absence, ils font sauter leur concierge et leur maison. Ce n'est qu'un début: la guerre civile est prévue pour l'automne ou pour le prochain printemps. Nos lobes pourtant semblent en parfait état : ne serait-ce pas plutôt que, faute de pouvoir écraser l'indigène, la violence revient sur soi, s'accumule au fond de nous et cherche une issue? L'union du peuple algérien produit la désunion du peuple français: sur tout le territoire de l'ex-métropole, les tribus dansent et se préparent [35] au combat. La terreur a quitté I'Afrique pour s'installer ici : car il y a des furieux tout bonnement, qui veulent nous faire payer de notre sang la honte d'avoir été battus par l'indigène et puis il y a les autres, tous les autres, aussi coupables - après Bizerte, après les lynchages de septembre, qui donc est descendu dans la rue pour dire: assez? - mais plus rassis : les libéraux, les durs de durs de la gauche molle. En eux aussi la fièvre monte. Et la hargne. Mais quelle frousse! Ils se masquent leur rage par des mythes, par des rites compliqués: pour retarder le règlement de comptes final et I'heure de la vérité, ils ont mis à notre tête un Grand Sorcier dont 
l'office est de nous maintenir à tout prix dans l'obscurité. Rien n'y fait : proclamée par les uns, refoulée par les autres, la violence tourne en rond: un jour elle explose à Metz, le lendemain à Bordeaux; elle a passé par ici, elle passera par là, c'est le jeu du furet. À notre tour, pas à pas, nous faisons le chemin qui mène à l'indigénat. Mais pour devenir indigènes tout à fait, il faudrait que notre sol fût occupé par les anciens colonisés et que nous crevions de faim. Ce ne sera pas: non, c'est le colonialisme déchu qui nous possède, c'est lui qui nous chevauchera bientôt, gâteux et superbe: le voilà, notre zar, notre loa. Et vous vous persuaderez en lisant le dernier chapitre de Fanon, qu'il vaut mieux être un indigène au pire moment de la misère qu'un ci-devant colon. Il n'est pas bon qu'un fonctionnaire de la police soit obligé de torturer dix heures par jour : à ce train-là, ses nerfs vont craquer à moins qu'on n'interdise aux bourreaux, dans leur propre intérêt, de faire des heures supplémentaires. Quand on veut protéger par la rigueur des lois le moral de la Nation et de l'Armée, il n'est pas bon que celle-ci démoralise systématiquement celle-là. Ni qu'un pays de tradition républicaine confie, par centaines de milliers, ses jeunes gens à des officiers putschistes. Il n'est pas bon, mes compatriotes, vous qui connaissez tous les crimes commis en notre nom, il n'est vraiment pas bon que vous n'en souffliez mot à personne, pas même à votre âme par crainte d'avoir à vous juger. Au début vous ignoriez, je veux le croire, ensuite vous avez douté, à présent vous savez mais vous vous [36] taisez toujours. Huit ans de silence, ça dégrade. Et vainement : aujourd'hui, l'aveuglant soleil de la torture est au zénith, il éclaire tout le pays: sous cette lumière, il n'y a plus un rire qui sonne juste, plus un visage qui ne se farde pour masquer la colère ou la peur, plus un acte qui ne trahisse nos dégoûts et nos complicités. Il suffit aujourd'hui que deux Français se rencontrent pour qu'il y ait un cadavre entre eux. Et quand je dis: un... La France, autrefois, c'était un nom de pays: prenons garde que ce ne soit, en 1961, le nom d'une névrose.

Guérirons-nous? Oui. La violence, comme la lance d'Achille, peut cicatriser les blessures qu'elle a faites. Aujourd' hui, nous sommes enchaînés, humiliés, malades de peur : au plus bas. Heureusement cela ne 
suffit pas encore à l'aristocratie colonialiste: elle ne peut accomplir sa mission retardatrice en Algérie qu'elle n'ait achevé d'abord de coloniser les Français. Nous reculons chaque jour devant la bagarre mais soyez sûrs que nous ne l'éviterons pas: ils en ont besoin, les tueurs: ils vont nous voler dans les plumes et taper dans le tas. Ainsi finira le temps des sorciers et des fétiches: il faudra vous battre ou pourrir dans les camps. C'est le dernier moment de la dialectique: vous condamnez cette guerre mais n'osez pas encore vous déclarer solidaires des combattants algériens: n'ayez crainte, comptez sur les colons et sur les mercenaires: ils vous feront sauter le pas. Peut-être, alors, le dos au mur, débriderez-vous enfin cette violence nouvelle que suscitent en vous de vieux forfaits recuits. Mais ceci, comme on dit, est une autre histoire. Celle de I'homme. Le temps s'approche, j'en suis sûr, où nous nous joindrons à ceux qui la font.

Jean-Paul SARTRE

septembre 1961 
[37]

\section{LES DAMNÉS DE LA TERRE}

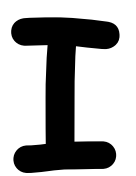

Retour à la table des matières 


\title{
LES DAMNÉS DE LA TERRE
}

\author{
I \\ De la violence
}

\section{Retour à la table des matières}

Libération nationale, renaissance nationale, restitution de la nation au peuple, Commonwealth, quelles que soient les rubriques utilisées ou les formules nouvelles introduites, la décolonisation est toujours un phénomène violent. À quelque niveau qu' on l'étudie: rencontres interindividuelles, appellations nouvelles des clubs sportifs, composition humaine des cocktails-parties, de la police, de conseils d'administration des banques nationales ou privées, la décolonisation est très simplement le remplacement d'une «espèce» d'hommes par une autre « espèce » d'hommes. Sans transition, il y a substitution totale, complète, absolue. Certes, on pourrait également montrer le surgissement d'une nouvelle nation, l'installation d'un État nouveau, ses relations diplomatiques, son orientation politique, économique. Mais nous avons précisément choisi de parler de cette sorte de table rase qui définit au départ toute décolonisation. Son importance inhabituelle est qu'elle constitue, dès le premier jour, la revendication minimum du colonisé. À vrai dire, la preuve du succès réside dans un panorama social changé de fond en comble. L'importance extraordinaire de ce changement est 
qu'il est voulu, réclamé, exigé. La nécessité de ce changement existe à l'état brut, impétueux et contraignant, dans la conscience et dans la vie des hommes et des femmes colonisés. Mais l'éventualité de ce changement est également vécue sous la forme d'un avenir terrifiant dans la conscience d'une autre «espèce» d'hommes et de femmes: les colons.

La décolonisation, qui se propose de changer l'ordre du monde, est, on le voit, un programme de désordre absolu. Mais [40] elle ne peut être le résultat d'une opération magique, d'une secousse naturelle ou d'une entente à l'amiable. La décolonisation, on le sait, est un processus historique : c'est-à-dire qu'elle ne peut être comprise, qu'elle ne trouve son intelligibilité, ne devient translucide à elle-même que dans l'exacte mesure où l'on discerne le mouvement historicisant qui lui donne forme et contenu. La décolonisation est la rencontre de deux forces congénitalement antagonistes qui tirent précisément leur originalité de cette sorte de substantification que sécrète et qu'alimente la situation coloniale. Leur première confrontation s'est déroulée sous le signe de la violence et leur cohabitation - plus précisément l'exploitation du colonisé par le colon - s'est poursuivie à grand renfort de baiionnettes et de canons. Le colon et le colonisé sont de vieilles connaissances. Et, de fait, le colon a raison quand il dit « les » connaître. C'est le colon qui a fait et qui continue à faire le colonisé. Le colon tire sa vérité, c'est-à-dire ses biens, du système colonial.

La décolonisation ne passe jamais inaperçue car elle porte sur l'être, elle modifie fondamentalement l'être, elle transforme des spectateurs écrasés d'inessentialité en acteurs privilégiés, saisis de façon quasi grandiose par le faisceau de l'Histoire. Elle introduit dans l'être un rythme propre, apporté par les nouveaux hommes, un nouveau langage, une nouvelle humanité. La décolonisation est véritablement création d'hommes nouveaux. Mais cette création ne reçoit sa légiti- 
mité d'aucune puissance surnaturelle : la «chose » colonisée devient homme dans le processus même par lequel elle se libère.

Dans décolonisation, il y a donc exigence d'une remise en question intégrale de la situation coloniale. Sa définition peut, si on veut la décrire avec précision, tenir dans la phrase bien connue: «Les derniers seront les premiers. " La décolonisation est la vérification de cette phrase. C'est pourquoi, sur le plan de la description, toute décolonisation est une réussite.

Présentée dans sa nudité, la décolonisation laisse deviner à travers tous ses pores, des boulets rouges, des couteaux sanglants. [41] Car si les derniers doivent être les premiers, ce ne peut être qu'à la suite d'un affrontement décisif et meurtrier des deux protagonistes. Cette volonté affirmée de faire remonter les derniers en tête de file, de les faire grimper (à une cadence trop rapide, disent certains) les fameux échelons qui définissent une société organisée, ne peut triompher que si on jette dans la balance tous les moyens, y compris, bien sûr, la violence.

On ne désorganise pas une société, aussi primitive soit-elle, avec un tel programme si l'on n'est pas décidé dès le début, c'est-à-dire dès la formulation même de ce programme, à briser tous les obstacles qu'on rencontrera sur sa route. Le colonisé qui décide de réaliser ce programme, de s'en faire le moteur, est préparé de tout temps à la violence. Dès sa naissance il est clair pour lui que ce monde rétréci, semé d'interdictions, ne peut être remis en question que par la violence absolue.

Le monde colonial est un monde compartimenté. Sans doute est-il superflu, sur le plan de la description, de rappeler l'existence de villes indigènes et de villes européennes, d'écoles pour indigènes et d'écoles pour Européens, comme il est superflu de rappeler l'apartheid en Afrique du Sud. Pourtant, si nous pénétrons dans l'intimité de cette compartimentation, nous aurons au moins le bénéfice de mettre en éviden- 
ce quelques-unes des lignes de force qu'elle comporte. Cette approche du monde colonial, de son arrangement, de sa disposition géographique va nous permettre de délimiter les arêtes à partir desquelles se réorganisera la société décolonisée.

Le monde colonisé est un monde coupé en deux. La ligne de partage, la frontière en est indiquée par les casernes et les postes de police. Aux colonies, l'interlocuteur valable et institutionnel du colonisé, le porte-parole du colon et du régime d'oppression est le gendarme ou le soldat. Dans les sociétés de type capitaliste, l'enseignement, religieux ou laique, la formation de réflexes moraux transmissibles de père en fils, I'honnêteté exemplaire d'ouvriers décorés après cinquante années de bons et loyaux services, l'amour encouragé de l'harmonie et de la [42] sagesse, ces formes esthétiques du respect de l'ordre établi, créent autour de l'exploité une atmosphère de soumission et d'inhibition qui allège considérablement la tâche des forces de l'ordre. Dans les pays capitalistes, entre l'exploité et le pouvoir s'interposent une multitude de professeurs de morale, de conseillers, de « désorientateurs». Dans les régions coloniales, par contre, le gendarme et le soldat, par leur présence immédiate, leurs interventions directes et fréquentes, maintiennent le contact avec le colonisé et lui conseillent, à coups de crosse ou de napalm, de ne pas bouger. On le voit, l'intermédiaire du pouvoir utilise un langage de pure violence. L'intermédiaire n'allège pas l'oppression, ne voile pas la domination. Il les expose, les manifeste avec la bonne conscience des forces de l'ordre. L'intermédiaire porte la violence dans les maisons et dans les cerveaux du colonisé.

La zone habitée par les colonisés n'est pas complémentaire de la zone habitée par les colons. Ces deux zones s'opposent, mais non au service d'une unité supérieure. Régies par une logique purement aristotélicienne, elles obéissent au principe d'exclusion réciproque : il n'y a pas de conciliation possible, l'un des termes est de trop. La ville du colon est une ville en dur, toute de pierre et de fer. C'est une ville illuminée, asphaltée, où les poubelles regorgent toujours de restes in- 
connus, jamais vus, même pas rêvés. Les pieds du colon ne sont jamais aperçus, sauf peut-être dans la mer, mais on n'est jamais assez proche d'eux. Des pieds protégés par des chaussures solides alors que les rues de leur ville sont nettes, lisses, sans trous, sans cailloux. La ville du colon est une ville repue, paresseuse, son ventre est plein de bonnes choses à l'état permanent. La ville du colon est une ville de blancs, d'étrangers.

La ville du colonisé, ou du moins la ville indigène, le village nègre, la médina, la réserve est un lieu mal famé, peuplé d'hommes mal famés. On y naît n'importe où, n'importe comment. On y meurt n'importe où, de n'importe quoi. C'est un monde sans intervalles, les hommes y sont les uns sur les autres, [43] les cases les unes sur les autres. La ville du colonisé est une ville affamée, affamée de pain, de viande, de chaussures, de charbon, de lumière. La ville du colonisé est une ville accroupie, une ville à genoux, une ville vautrée. C'est une ville de nègres, une ville de bicots. Le regard que le colonisé jette sur la ville du colon est un regard de luxure, un regard d'envie. Rêves de possession. Tous les modes de possession : s'asseoir à la table du colon, coucher dans le lit du colon, avec sa femme si possible. Le colonisé est un envieux. Le colon ne l'ignore pas qui, surprenant son regard à la dérive, constate amèrement mais toujours sur le qui-vive: "Ils veulent prendre notre place. » C'est vrai, il n'y a pas un colonisé qui ne rêve au moins une fois par jour de s'installer à la place du colon.

Ce monde compartimenté, ce monde coupé en deux est habité par des espèces différentes. L'originalité du contexte colonial, c'est que les réalités économiques, les inégalités, l'énorme différence des modes de vie ne parviennent jamais à masquer les réalités humaines. Quand on aperçoit dans son immédiateté le contexte colonial, il est patent que ce qui morcelle le monde c'est d'abord le fait d'appartenir ou non à telle espèce, à telle race. Aux colonies, l'infrastructure économique est également une superstructure. La cause est conséquence : on est riche parce que blanc, on est blanc parce que riche. C'est pourquoi les analyses marxistes doivent être toujours légèrement distendues chaque fois qu'on aborde le problème colonial. Il n'y a pas jus- 
qu'au concept de société précapitaliste, bien étudié par Marx, qui ne demanderait ici à être repensé. Le serf est d'une essence autre que le chevalier, mais une référence au droit divin est nécessaire pour légitimer cette différence statutaire. Aux colonies, l'étranger venu d'ailleurs s'est imposé à l'aide de ses canons et de ses machines. En dépit de la domestication réussie, malgré l'appropriation le colon reste toujours un étranger. Ce ne sont ni les usines, ni les propriétés, ni le compte en banque qui caractérisent d'abord la «classe dirigeante». L'espèce dirigeante est d'abord celle qui vient d'ailleurs, celle qui ne ressemble pas aux autochtones, « les autres».

[44]

La violence qui a présidé à l'arrangement du monde colonial, qui a rythmé inlassablement la destruction des formes sociales indigènes, démoli sans restrictions les systèmes de références de l'économie, les modes d'apparence, d'habillement, sera revendiquée et assumée par le colonisé au moment où, décidant d'être l'histoire en actes, la masse colonisée s'engouffrera dans les villes interdites. Faire sauter le monde colonial est désormais une image d'action très claire, très compréhensible et pouvant être reprise par chacun des individus constituant le peuple colonisé. Disloquer le monde colonial ne signifie pas qu'après l'abolition des frontières on aménagera des voies de passage entre les deux zones. Détruire le monde colonial c'est ni plus ni moins abolir une zone, l'enfouir au plus profond du sol ou l'expulser du territoire.

La mise en question du monde colonial par le colonisé n'est pas une confrontation rationnelle des points de vue. Elle n'est pas un discours sur l'universel, mais l'affirmation échevelée d'une originalité posée comme absolue. Le monde colonial est un monde manichéiste. Il ne suffit pas au colon de limiter physiquement, c'est-à-dire à l'aide de sa police et de sa gendarmerie, l'espace du colonisé. Comme pour illustrer le caractère totalitaire de l'exploitation coloniale, le colon fait du co- 
lonisé une sorte de quintessence du mal ${ }^{1}$. La société colonisée n'est pas seulement décrite comme une société sans valeurs. Il ne suffit pas au colon d'affirmer que les valeurs ont déserté, ou mieux $n$ 'ont jamais habité, le monde colonisé. L'indigène est déclaré imperméable à l'éthique, absence de valeurs, mais aussi négation des valeurs. Il est, osons l'avouer, l'ennemi des valeurs. En ce sens, il est le mal absolu. Élément corrosif, détruisant tout ce qui l'approche, élément déformant, défigurant tout ce qui a trait à l'esthétique ou à la morale, dépositaire de forces maléfiques, instrument inconscient et irrécupérable de forces aveugles. Et [45] M. Meyer pouvait dire sérieusement à l'Assemblée nationale française qu'il ne fallait pas prostituer la République en y faisant pénétrer le peuple algérien. Les valeurs, en effet, sont irréversiblement empoisonnées et infectées dès lors qu'on les met en contact avec le peuple colonisé. Les coutumes du colonisé, ses traditions, ses mythes, surtout ses mythes, sont la marque même de cette indigence, de cette dépravation constitutionnelle. C'est pourquoi il faut mettre sur le même plan le DDT qui détruit les parasites, vecteurs de maladie, et la religion chrétienne qui combat dans l'œuf les hérésies, les instincts, le mal. Le recul de la fièvre jaune et les progrès de l'évangélisation font partie du même bilan. Mais les communiqués triomphants des missions renseignent en réalité sur l'importance des ferments d'aliénation introduits au sein du peuple colonisé. Je parle de la religion chrétienne, et personne n'a le droit de s'en étonner. L'Église aux colonies est une Église de Blancs, une église d'étrangers. Elle n'appelle pas I'homme colonisé dans la voie de Dieu mais bien dans la voie du Blanc, dans la voie du maître, dans la voie de l'oppresseur. Et comme on le sait, dans cette histoire il y a beaucoup d'appelés et peu d'élus.

Parfois ce manichéisme va jusqu'au bout de sa logique et déshumanise le colonisé. À proprement parler, il l'animalise. Et, de fait, le langage du colon, quand il parle du colonisé, est un langage zoologique. On fait allusion aux mouvements de reptation du jaune, aux émanations de

1 Nous avons montré dans Peau noire, Masques blancs (éditions du Seuil) le mécanisme de ce monde manichéiste. 
la ville indigène, aux hordes, à la puanteur, au pullulement, au grouillement, aux gesticulations. Le colon, quand il veut bien décrire et trouver le mot juste, se réfère constamment au bestiaire. L'Européen bute rarement sur les termes «imagés ». Mais le colonisé, qui saisit le projet du colon, le procès précis qu' on lui intente, sait immédiatement à quoi l'on pense. Cette démographie galopante, ces masses hystériques, ces visages d'où toute humanité a fui, ces corps obèses qui ne ressemblent plus à rien, cette cohorte sans tête ni queue, ces enfants qui ont l'air de n'appartenir à personne, cette paresse étalée sous le soleil, ce rythme végétal, tout cela fait partie du vocabulaire colonial. Le général de Gaulle parle des [46] « multitudes jaunes » et M. Mauriac des masses noires, brunes et jaunes qui bientôt vont déferler. Le colonisé sait tout cela et rit un bon coup chaque fois qu'il se découvre animal dans les paroles de l'autre. Car il sait qu'il n'est pas un animal. Et précisément, dans le même temps qu'il découvre son humanité, il commence à fourbir ses armes pour la faire triompher.

Dès que le colonisé commence à peser sur ses amarres, à inquiéter le colon, on lui délègue de bonnes âmes qui, dans les «Congrès de culture », lui exposent la spécificité, les richesses des valeurs occidentales. Mais chaque fois qu'il est question de valeurs occidentales il se produit, chez le colonisé, une sorte de raidissement, de tétanie musculaire. Dans la période de décolonisation, il est fait appel à la raison des colonisés. On leur propose des valeurs sûres, on leur explique abondamment que la décolonisation ne doit pas signifier régression, qu'il faut s'appuyer sur des valeurs expérimentées, solides, cotées. Or il se trouve que lorsqu'un colonisé entend un discours sur la culture occidentale, il sort sa machette ou du moins il s'assure qu'elle est à portée de sa main. La violence avec laquelle s'est affirmée la suprématie des valeurs blanches, l'agressivité qui a imprégné la confrontation victorieuse de ces valeurs avec les modes de vie ou de pensée des colonisés font que, par un juste retour des choses, le colonisé ricane quand on évoque devant lui ces valeurs. Dans le contexte colonial, le colon ne s'arrête dans son travail d'éreintement du colonisé que lors- 
que ce dernier a reconnu à haute et intelligible voix la suprématie des valeurs blanches. Dans la période de décolonisation, la masse colonisée se moque de ces mêmes valeurs, les insulte, les vomit à pleine gorge.

Ce phénomène est d'ordinaire masqué parce que, pendant la période de décolonisation, certains intellectuels colonisés ont établi un dialogue avec la bourgeoisie du pays colonialiste. Pendant cette période, la population autochtone est perçue comme masse indistincte. Les quelques individualités indigènes [47] que les bourgeois colonialistes ont eu l'occasion de connaître çà et là ne pèsent pas suffisamment sur cette perception immédiate pour donner naissance à des nuances. Par contre, pendant la période de libération, la bourgeoisie colonialiste cherche avec fièvre des contacts avec les "élites». C'est avec ces élites qu'est entrepris le fameux dialogue sur les valeurs. La bourgeoisie colonialiste, quand elle enregistre l'impossibilité pour elle de maintenir sa domination sur les pays coloniaux, décide de mener un combat d'arrière-garde sur le terrain de la culture, des valeurs, des techniques, etc. Or, ce qu'il ne faut jamais perdre de vue, c'est que l'immense majorité des peuples colonisés est imperméable à ces problèmes. Pour le peuple colonisé la valeur la plus essentielle, parce que la plus concrète, c'est d'abord la terre : la terre qui doit assurer le pain et, bien sûr, la dignité. Mais cette dignité n'a rien à voir avec la dignité de la «personne humaine ». Cette personne humaine idéale, il n'en a jamais entendu parler. Ce que le colonisé a vu sur son sol, c'est qu' on pouvait impunément l'arrêter, le frapper, l'affamer; et aucun professeur de morale jamais, aucun curé jamais n'est venu recevoir les coups à sa place ni partager son pain avec lui. Pour le colonisé, être moraliste c'est, très concrètement, faire taire la morgue du colon, briser sa violence étalée, en un mot l'expulser carrément du panorama. Le fameux principe qui veut que tous les hommes soient égaux trouvera son illustration aux colonies dès lors que le colonisé posera qu'il est l'égal du colon. Un pas de plus, il voudra se battre pour être plus que le colon. En fait, il a déjà décidé de remplacer le colon, de prendre sa place. Comme on le voit, c'est tout un univers matériel et moral qui s'écroule. 
L'intellectuel qui a, pour sa part, suivi le colonialiste sur le plan de l'universel abstrait va se battre pour que colon et colonisé puissent vivre en paix dans un monde nouveau. Mais ce qu'il ne voit pas, parce que précisément le colonialisme s'est infiltré en lui avec tous ses modes de pensée, c'est que le colon, dès lors que le contexte colonial disparaît, n'a plus d'intérêt à rester, à coexister. Ce n'est pas un hasard si, avant même toute négociation entre le gouvernement algérien et le gouvernement [48] français, la minorité européenne dite « libérale » a déjà fait connaître sa position: elle réclame, ni plus ni moins, la double citoyenneté. C'est qu'en se cantonnant sur le plan abstrait on veut condamner le colon à effectuer un saut très concret dans l'inconnu. Disons-le, le colon sait parfaitement qu'aucune phraséologie ne se substitue au réel.

Le colonisé, donc, découvre que sa vie, sa respiration, les battements de son cœur sont les mêmes que ceux du colon. Il découvre qu'une peau de colon ne vaut pas plus qu'une peau d'indigène. C'est dire que cette découverte introduit une secousse essentielle dans le monde. Toute l'assurance nouvelle et révolutionnaire du colonisé en découle. Si, en effet, ma vie a le même poids que celle du colon, son regard ne me foudroie plus, ne m'immobilise plus, sa voix ne me pétrifie plus. Je ne me trouble plus en sa présence. Pratiquement, je l'emmerde. Non seulement sa présence ne me gêne plus, mais déjà je suis en train de lui préparer de telles embuscades qu'il n'aura bientôt d'autre issue que la fuite.

Le contexte colonial, avons-nous dit, se caractérise par la dichotomie qu'il inflige au monde. La décolonisation unifie ce monde en lui enlevant par une décision radicale son hétérogénéité, en l'unifiant sur la base de la nation, quelquefois de la race. On connaît ce mot féroce des patriotes sénégalais évoquant les mancuvres de leur président Senghor: «Nous avons demandé l'africanisation des cadres, et voici que Senghor africanise les Européens. » Ce qui veut dire que le colonisé a la possibilité de percevoir dans une immédiateté absolue si la décoloni- 
sation a lieu ou non: le minimum exigé étant que les derniers deviennent les premiers.

Mais l'intellectuel colonisé apporte des variantes à cette pétition et, de fait, les motivations ne semblent pas lui manquer: cadres administratifs, cadres techniques, spécialistes. Or le colonisé interprète ces passe-droits comme autant de manœuvres de sabotage et il n'est pas rare d'entendre, çà et là, un colonisé déclarer: «Ce n'était pas la peine, alors, d'être indépendants... »

[49]

Dans les régions colonisées où une véritable lutte de libération a été menée, où le sang du peuple a coulé et où la durée de la phase armée a favorisé le reflux des intellectuels sur des bases populaires, on assiste à une véritable éradication de la superstructure puisée par ces intellectuels dans les milieux bourgeois colonialistes. Dans son monologue narcissiste, la bourgeoisie colonialiste, par l'intermédiaire de ses universitaires, avait profondément ancré en effet dans l'esprit du colonisé que les essences demeurent éternelles en dépit de toutes les erreurs imputables aux hommes. Les essences occidentales s'entend. Le colonisé acceptait le bien-fondé de ces idées et l'on pouvait découvrir, dans un repli de son cerveau, une sentinelle vigilante chargée de défendre le socle gréco-latin. Or il se trouve que, pendant la lutte de libération, au moment où le colonisé reprend contact avec son peuple, cette sentinelle factice est pulvérisée. Toutes les valeurs méditerranéennes, triomphe de la personne humaine, de la clarté et du Beau, deviennent des bibelots sans vie et sans couleur. Tous ces discours apparaissent comme des assemblages de mots morts. Ces valeurs qui semblaient ennoblir l'âme se révèlent inutilisables parce qu'elles ne concernent pas le combat concret dans lequel le peuple s'est engagé.

Et d'abord l'individualisme. L'intellectuel colonisé avait appris de ses maîtres que l'individu doit s'affirmer. La bourgeoisie colonialiste avait enfoncé à coups de pilon dans l'esprit du colonisé l'idée d'une société d'individus où chacun s'enferme dans sa subjectivité, où la richesse est celle de la pensée. Or le colonisé qui aura la chance de 
s'enfouir dans le peuple pendant la lutte de libération va découvrir la fausseté de cette théorie. Les formes d'organisation de la lutte vont déjà lui proposer un vocabulaire inhabituel. Le frère, la sœur, le camarade sont des mots proscrits par la bourgeoisie colonialiste parce que pour elle mon frère c'est mon portefeuille, mon camarade c'est ma combine. L'intellectuel colonisé assiste, dans une sorte d'autodafé, à la destruction de toutes ses idoles: l'égoïsme, la récrimination orgueilleuse, l'imbécillité infantile de celui qui veut toujours avoir le dernier mot. Cet intellectuel colonisé, atomisé [50] par la culture colonialiste, découvrira également la consistance des assemblées de villages, la densité des commissions du peuple, l'extraordinaire fécondité des réunions de quartier et de cellule. L'affaire de chacun ne cesse plus désormais d'être l'affaire de tous parce que, concrètement, on sera tous découverts par les légionnaires, donc massacrés, ou on sera tous sauvés. Le «démerdage», cette forme athée du salut, est, dans ce contexte, prohibé.

On parle beaucoup, depuis quelque temps, de l'autocritique: mais sait-on que c'est d'abord une institution africaine? Que ce soit dans les djemaas d'Afrique du Nord ou dans les réunions d'Afrique occidentale, la tradition veut que les conflits qui éclatent dans un village soient débattus en public. Autocritique en commun bien sûr, avec cependant une note d'humour parce que tout le monde est détendu, parce que nous voulons tous en dernier ressort les mêmes choses. Le calcul, les silences insolites, les arrière-pensées, l'esprit souterrain, le secret, tout cela l'intellectuel l'abandonne au fur et à mesure de sa plongée dans le peuple. Et il est vrai qu' on peut dire alors que la communauté triomphe déjà à ce niveau, qu'elle sécrète sa propre lumière, sa propre raison.

Mais il arrive que la décolonisation ait lieu dans des régions qui n'ont pas été suffisamment secouées par la lutte de libération et l'on retrouve ces mêmes intellectuels débrouillards, malins, astucieux. On retrouve chez eux, intactes, les conduites et les formes de pensée ramassées au cours de leur fréquentation de la bourgeoisie colonialiste. Enfants gâtés hier du colonialisme, aujourd'hui de l'autorité natio- 
nale, ils organisent le pillage des quelques ressources nationales. Impitoyables, ils se hissent par les combines ou les vols légaux: importexport, sociétés anonymes, jeux de bourse, passe-droits, sur cette misère aujourd' hui nationale. Ils demandent avec insistance la nationalisation des affaires commerciales, c'est-à-dire la réservation des marchés et des bonnes occasions aux seuls nationaux. Doctrinalement, ils proclament la nécessité impérieuse de nationaliser le vol de la nation. Dans cette aridité de la période nationale, [51] dans la phase dite d'austérité, le succès de leurs rapines provoque rapidement la colère et la violence du peuple. Ce peuple misérable et indépendant, dans le contexte africain et international actuel, accède à la conscience sociale à une cadence accélérée. Cela, les petites individualités ne tarderont pas à le comprendre.

Pour assimiler la culture de l'oppresseur et s'y aventurer, le colonisé a dû fournir des gages. Entre autres, il a dû faire siennes les formes de pensée de la bourgeoisie coloniale. Cela, on le constate dans l'inaptitude de l'intellectuel colonisé à dialoguer. Car il ne sait pas se faire inessentiel en face de l'objet ou de l'idée. Par contre, quand il milite au sein du peuple il va d'émerveillement en émerveillement. Il est littéralement désarmé par la bonne foi et par l'honnêteté du peuple. Le risque permanent qui le guette est alors de faire du populisme. Il se transforme en une sorte de béni-oui-oui qui opine à chaque phrase du peuple, transformé par lui en sentence. Or le fellah, le chômeur, l'affamé, ne prétend pas à la vérité. Il ne dit pas qu'il est la vérité, car il l'est dans son être même.

L'intellectuel se comporte objectivement, dans cette période, comme un vulgaire opportuniste. Ses manœuvres, en fait, n'ont pas cessé. Il n'est pas question pour le peuple, jamais, de le repousser ou de l'acculer. Ce que le peuple demande, c'est qu' on mette tout en commun. L'insertion de l'intellectuel colonisé dans la marée populaire va se trouver différée par l'existence chez lui d'un curieux culte du détail. Ce n'est pas que le peuple soit rebelle à l'analyse. Il aime qu'on lui explique, il aime comprendre les articulations d'un raisonnement, il aime 
voir où il va. Mais l'intellectuel colonisé, au début de sa cohabitation avec le peuple, privilégie le détail et en arrive à oublier la défaite du colonialisme, l'objet même de la lutte. Emporté dans le mouvement multiforme de la lutte, il a tendance à se fixer sur des tâches locales, poursuivies avec ardeur mais presque toujours trop solennisées. Il ne voit pas tout le temps le tout. Il introduit la notion de disciplines, de spécialités, de [52] domaines, dans cette terrible machine à mélanger et à concasser qu'est une révolution populaire. Engagé sur des points précis du front, il lui arrive de perdre de vue l'unité du mouvement et, en cas d'échec local, de se laisser aller au doute, voire au désespoir. Le peuple, par contre, adopte dès le départ des positions globales. La terre et le pain: que faire pour avoir la terre et le pain? Et cet aspect buté, apparemment limité, rétréci, du peuple, est en définitive le modèle opératoire le plus enrichissant et le plus efficace.

Le problème de la vérité doit également retenir notre attention. Au sein du peuple, de tout temps, la vérité n'est due qu'aux nationaux. Aucune vérité absolue, aucun discours sur la transparence de l'âme ne peut effriter cette position. Au mensonge de la situation coloniale, le colonisé répond par un mensonge égal. La conduite est ouverte avec les nationaux, crispée et illisible avec les colons. Le vrai, c'est ce qui précipite la dislocation du régime colonial, c'est ce qui favorise l'émergence de la nation. Le vrai, c'est ce qui protège les indigènes et perd les étrangers. Dans le contexte colonial il n'y a pas de conduite de vérité. Et le bien est tout simplement ce qui leur fait du mal.

On voit donc que le manichéisme premier qui régissait la société coloniale est conservé intact dans la période de décolonisation. C'est que le colon ne cesse jamais d'être l'ennemi, l'antagoniste, très précisément I'homme à abattre. L'oppresseur, dans sa zone, fait exister le mouvement, mouvement de domination, d'exploitation, de pillage. Dans l'autre zone, la chose colonisée lovée, pillée, alimente comme elle peut ce mouvement, qui va sans transition de la berge du territoire aux pa- 
lais et aux docks de la «métropole». Dans cette zone figée, la surface est étale, le palmier se balance devant les nuages, les vagues de la mer ricochent sur les galets, les matières premières vont et viennent, légitimant la présence du colon, tandis qu'accroupi, plus mort que vif, le colonisé s'éternise dans un rêve toujours le même. Le colon fait I'histoire. Sa vie est une épopée, une odyssée. Il est le [53] commencement absolu: "Cette terre, c'est nous qui l'avons faite. » Il est la cause continuée: "Si nous partons, tout est perdu, cette terre retournera au Moyen Âge. » En face de lui, des êtres engourdis, travaillés de l'intérieur par les fièvres et les « coutumes ancestrales», constituent un cadre quasi minéral au dynamisme novateur du mercantilisme colonial.

Le colon fait I'histoire et sait qu'il la fait. Et parce qu'il se réfère constamment à l'histoire de sa métropole, il indique en clair qu'il est ici le prolongement de cette métropole. L'histoire qu'il écrit n'est donc pas I'histoire du pays qu'il dépouille mais l'histoire de sa nation en ce qu'elle écume, viole et affame. L'immobilité à laquelle est condamné le colonisé ne peut être remise en question que si le colonisé décide de mettre un terme à l'histoire de la colonisation, à l'histoire du pillage, pour faire exister I'histoire de la nation, I'histoire de la décolonisation.

Monde compartimenté, manichéiste, immobile, monde de statues : la statue du général qui a fait la conquête, la statue de l'ingénieur qui a construit le pont. Monde sûr de lui, écrasant de ses pierres les échines écorchées par le fouet. Voilà le monde colonial. L'indigène est un être parqué, l'apartheid n'est qu'une modalité de la compartimentation du monde colonial. La première chose que l'indigène apprend, c'est à rester à sa place, à ne pas dépasser les limites. C'est pourquoi les rêves de l'indigène sont des rêves musculaires, des rêves d'action, des rêves agressifs. Je rêve que je saute, que je nage, que je cours, que je grimpe. Je rêve que j'éclate de rire, que je franchis le fleuve d'une enjam- 
bée, que je suis poursuivi par des meutes de voitures qui ne me rattrapent jamais. Pendant la colonisation, le colonisé n'arrête pas de se libérer entre neuf heures du soir et six heures du matin.

Cette agressivité sédimentée dans ses muscles, le colonisé va la manifester d'abord contre les siens. C'est la période où les nègres se bouffent entre eux et où les policiers, les juges d'instruction ne savent plus où donner de la tête devant l'étonnante [54] criminalité nord-africaine. Nous verrons plus loin ce qu'il faut penser de ce phénomène ${ }^{2}$. Face à l'arrangement colonial le colonisé se trouve dans un état de tension permanente. Le monde du colon est un monde hostile, qui rejette, mais dans le même temps c'est un monde qui fait envie. Nous avons vu que le colonisé rêve toujours de s'installer à la place du colon. Non pas de devenir un colon, mais de se substituer au colon. Ce monde hostile, pesant, agressif, parce que repoussant de toutes ses aspérités la masse colonisée, représente non pas l'enfer duquel on voudrait s'éloigner le plus rapidement possible mais un paradis à portée de main que protègent de terribles molosses.

Le colonisé est toujours sur le qui-vive car, déchiffrant difficilement les multiples signes du monde colonial, il ne sait jamais s'il a franchi ou non la limite. Face au monde arrangé par le colonialiste, le colonisé est toujours présumé coupable. La culpabilité du colonisé n'est pas une culpabilité assumée, c'est plutôt une sorte de malédiction, d'épée de Damoclès. Or, au plus profond de lui-même le colonisé ne reconnaît aucune instance. Il est dominé, mais non domestiqué. Il est infériorisé, mais non convaincu de son infériorité. Il attend patiemment que le colon relâche sa vigilance pour lui sauter dessus. Dans ses muscles, le colonisé est toujours en attente. On ne peut pas dire qu'il soit inquiet, qu'il soit terrorisé. En fait, il est toujours prêt à abandonner son rôle de gibier pour prendre celui de chasseur. Le colonisé est un persécuté qui rêve en permanence de devenir persécuteur. Les symboles sociaux - gendarmes, clairons sonnant dans les casernes,

2 Guerres coloniales et troubles mentaux, chapitre 5. 
défilés militaires et le drapeau là-haut - servent à la fois d'inhibiteurs et d'excitants. Ils ne signifient point : «Ne bouge pas», mais : «Prépare bien ton coup». Et, de fait, si le colonisé avait tendance à s'endormir, à oublier, la morgue du colon et son souci d'expérimenter la solidité du système colonial lui rappelleraient à maintes reprises que la grande [55] confrontation ne pourra être indéfiniment reportée. Cette impulsion à prendre la place du colon entretient un tonus musculaire de tous les instants. On sait, en effet, que dans des conditions émotionnelles données la présence de l'obstacle accentue la tendance au mouvement.

Les rapports colon-colonisé sont des rapports de masse. Au nombre, le colon oppose sa force. Le colon est un exhibitionniste. Son souci de sécurité l'amène à rappeler à haute voix au colonisé que "Le maître, ici, c'est moi ». Le colon entretient chez le colonisé une colère qu'il stoppe à la sortie. Le colonisé est pris dans les mailles serrées du colonialisme. Mais nous avons vu qu'à l'intérieur le colon n'obtient qu'une pseudo-pétrification. La tension musculaire du colonisé se libère périodiquement dans des explosions sanguinaires: luttes tribales, luttes de çofs, luttes entre individus.

Au niveau des individus, on assiste à une véritable négation du bon sens. Alors que le colon ou le policier peuvent, à longueur de journée, frapper le colonisé, l'insulter, le faire mettre à genoux, on verra le colonisé sortir son couteau au moindre regard hostile ou agressif d'un autre colonisé. Car la dernière ressource du colonisé est de défendre sa personnalité face à son congénère. Les luttes tribales ne font que perpétuer de vieilles rancunes enfoncées dans les mémoires. En se lançant à muscles perdus dans ses vengeances, le colonisé tente de se persuader que le colonialisme n'existe pas, que tout se passe comme avant, que I'histoire continue. Nous saisissons là en pleine clarté, au niveau des collectivités, ces fameuses conduites d'évitement, comme si la plongée dans ce sang fraternel permettait de ne pas voir l'obstacle, de renvoyer à plus tard l'option pourtant inévitable, celle qui débouche sur la lutte armée contre le colonialisme. Autodestruction col- 
lective très concrète dans les luttes tribales, telle est donc l'une des voies par où se libère la tension musculaire du colonisé. Tous ces comportements sont des réflexes de mort en face du danger, des conduites-suicides qui permettent au colon, dont la vie et la domination se trouvent [56] consolidées d'autant, de vérifier par la même occasion que ces hommes ne sont pas raisonnables. Le colonisé réussit également, par l'intermédiaire de la religion, à ne pas tenir compte du colon. Par le fatalisme, toute initiative est enlevée à l'oppresseur, la cause des maux, de la misère, du destin revenant à Dieu. L'individu accepte ainsi la dissolution décidée par Dieu, s'aplatit devant le colon et devant le sort et, par une sorte de rééquilibration intérieure, accède à une sérénité de pierre.

Entre-temps, cependant, la vie continue, et c'est à travers les mythes terrifiants, si prolifiques dans les sociétés sous-développées, que le colonisé va puiser des inhibitions à son agressivité : génies malfaisants qui interviennent chaque fois que l'on bouge de travers, hommesléopards, hommes-serpents, chiens à six pattes, zombies, toute une gamme inépuisable d'animalcules ou de géants dispose autour du colonisé un monde de prohibitions, de barrages, d'inhibitions beaucoup plus terrifiant que le monde colonialiste. Cette superstructure magique qui imprègne la société indigène remplit, dans le dynamisme de l'économie libidinale, des fonctions précises. L'une des caractéristiques, en effet, des sociétés sous-développées c'est que la libido est d'abord une affaire de groupe, de famille. On connaît ce trait, bien décrit par les ethnologues, de sociétés où l'homme qui rêve qu'il a des relations sexuelles avec une autre femme que la sienne doit avouer publiquement ce rêve et payer l'impôt en nature ou en journées de travail au mari ou à la famille lésée. Ce qui prouve, en passant, que les sociétés dites antéhistoriques attachent une grande importance à l'inconscient.

L'atmosphère de mythe et de magie, en me faisant peur, se comporte comme une réalité indubitable. En me terrifiant, elle m'intègre dans les traditions, dans I'histoire de ma contrée ou de ma tribu, mais dans le même temps elle me rassure, elle me délivre un statut, un bulletin d'état civil. Le plan du secret, dans les pays sous-développés, est un 
plan collectif relevant exclusivement de la magie. En me circonvenant dans ce lacis inextricable où les actes se répètent avec une permanence cristalline, c'est la pérennité d'un monde mien, d'un monde nôtre qui se [57] trouve ainsi affirmée. Les zombies, croyez-moi, sont plus terrifiants que les colons. Et le problème, dès lors, n'est plus de se mettre en règle avec le monde bardé de fer du colonialisme mais de réfléchir à trois fois avant d'uriner, de cracher ou de sortir dans la nuit.

Les forces surnaturelles, magiques, se révèlent être des forces étonnamment moïques. Les forces du colon sont infiniment rapetissées, frappées d'extranéité. On n'a plus vraiment à lutter contre elles puisque aussi bien ce qui compte c'est l'effrayante adversité des structures mythiques. Tout se résout, on le voit, en affrontement permanent sur le plan phantasmatique.

Toutefois, dans la lutte de libération, ce peuple autrefois réparti en cercles irréels, ce peuple en proie à un effroi indicible mais heureux de se perdre dans une tourmente onirique, se disloque, se réorganise et enfante dans le sang et les larmes des confrontations très réelles et très immédiates. Donner à manger aux moudjahidines, poster des sentinelles, venir en aide aux familles privées du nécessaire, se substituer au mari abattu ou emprisonné : telles sont les tâches concrètes auxquelles le peuple est convié dans la lutte de libération.

Dans le monde colonial, l'affectivité du colonisé est maintenue à fleur de peau comme une plaie vive qui fuit l'agent caustique. Et le psychisme se rétracte, s'oblitère, se décharge dans des démonstrations musculaires qui ont fait dire à des hommes très savants que le colonisé est un hystérique. Cette affectivité en érection, épiée par des gardiens invisibles mais qui communiquent sans transition avec le noyau de la personnalité, va se complaire avec érotisme dans les dissolutions motrices de la crise.

Sur un autre versant, nous verrons l'affectivité du colonisé s'épuiser en danses plus ou moins extatiques. C'est pourquoi une étude du 
monde colonial doit obligatoirement s'attacher à la compréhension du phénomène de la danse et de la possession. La relaxation du colonisé, c'est précisément cette orgie musculaire au cours de laquelle l'agressivité la plus aiguë, la violence [58] la plus immédiate se trouvent canalisées, transformées, escamotées. Le cercle de la danse est un cercle permissif. Il protège et autorise. À heures fixes, à dates fixes, hommes et femmes se retrouvent en un lieu donné et, sous l'œil grave de la tribu, se lancent dans une pantomime d'allure désordonnée mais en réalité très systématisée où, par des voies multiples, dénégations de la tête, courbure de la colonne, rejet en arrière de tout le corps, se déchiffre à livre ouvert l'effort grandiose d'une collectivité pour s'exorciser, s'affranchir, se dire. Tout est permis... dans le cercle. Le monticule où l'on s'est hissé comme pour être plus près de la lune, la berge où l'on s'est glissé comme pour manifester l'équivalence de la danse et de l'ablution, du lavage, de la purification sont des lieux sacrés. Tout est permis car, en réalité, l'on ne se réunit que pour laisser la libido accumulée, l'agressivité empêchée, sourdre volcaniquement. Mises à mort symboliques, chevauchées figuratives, meurtres multiples imaginaires, il faut que tout cela sorte. Les mauvaises humeurs s'écoulent, bruyantes telles des coulées de lave.

Un pas de plus et nous tombons en pleine possession. Au vrai, ce sont des séances de possession-dépossession qui sont organisées: vampirisme, possession par les djinns, par les zombies, par Legba, le Dieu illustre du vaudou. Ces effritements de la personnalité, ces dédoublements, ces dissolutions remplissent une fonction économique primordiale dans la stabilité du monde colonisé. À l'aller, les hommes et les femmes étaient impatients, piétinants, "sur les nerfs». Au retour, c'est le calme qui revient au village, la paix, l'immobilité.

On assistera au cours de la lutte de libération à une désaffection singulière pour ces pratiques. Le dos au mur, le couteau sur la gorge ou, pour être plus précis, l'électrode sur les parties génitales, le colonisé va être sommé de ne plus se raconter d'histoires.

Après des années d'irréalisme, après s'être vautré dans les phantasmes les plus étonnants, le colonisé, sa mitraillette au poing, affron- 
te enfin les seules forces qui lui contestaient son être: celles du colonialisme. Et le jeune colonisé qui grandit dans une atmosphère de fer et de feu peut bien se moquer - il ne [59] s'en prive pas - des ancêtres zombies, des chevaux à deux têtes, des morts qui se réveillent, des djinns qui profitent d'un bâillement pour s'engouffrer dans le corps. Le colonisé découvre le réel et le transforme dans le mouvement de sa praxis, dans l'exercice de la violence, dans son projet de libération.

Nous avons vu que cette violence, pendant toute la durée de la période coloniale, quoique à fleur de peau, tourne à vide. Nous l'avons vue canalisée par les décharges émotionnelles de la danse ou de la possession. Nous l'avons vue s'épuiser en luttes fratricides. Le problème se pose maintenant de saisir cette violence en train de se réorienter. Alors qu'elle se complaisait dans les mythes et qu'elle s'ingéniait à découvrir des occasions de suicide collectif, voici que des conditions nouvelles vont lui permettre de changer d'orientation.

Sur le plan de la tactique politique et de l'Histoire, un problème théorique d'une importance capitale est posé à l'époque contemporaine par la libération des colonies; quand peut-on dire que la situation est mûre pour un mouvement de libération nationale? Quelle doit en être l'avant-garde? Parce que les décolonisations ont revêtu des formes multiples, la raison hésite et s'interdit de dire ce qui est une vraie décolonisation et ce qui est une fausse décolonisation. Nous verrons, que, pour I'homme engagé, il y a urgence à décider des moyens, de la tactique, c'est-à-dire de la conduite et de l'organisation. Hors cela, il n'y a plus que volontarisme aveugle avec les aléas terriblement réactionnaires qu'il comporte.

Quelles sont les forces qui, dans la période coloniale, proposent à la violence du colonisé de nouvelles voies, de nouveaux pôles d'investissement? Ce sont d'abord les partis politiques et les élites intellectuelles ou commerciales. Or ce qui caractérise certaines formations 
politiques, c'est le fait qu'elles proclament des principes mais s'abstiennent de lancer des mots d'ordre. Toute l'activité de ces partis politiques nationalistes dans la [60] période coloniale est une activité de type électoraliste, c'est une suite de dissertations philosophicopolitiques sur le thème du droit des peuples à disposer d'eux-mêmes, du droit des hommes à la dignité et au pain, l'affirmation ininterrompue du principe "un homme-une voix». Les partis politiques nationalistes n'insistent jamais sur la nécessité de l'épreuve de force, parce que leur objectif n'est pas précisément le renversement radical du système. Pacifistes, légalistes, en fait partisans de l'ordre... nouveau, ces formations politiques posent crûment à la bourgeoisie colonialiste la question qui leur est essentielle: «Donnez-nous plus de pouvoir. » Sur le problème spécifique de la violence, les élites sont ambiguës. Elles sont violentes dans les paroles et réformistes dans les attitudes. Quand les cadres politiques nationalistes bourgeois disent une chose, ils signifient sans ambages qu'ils ne la pensent pas réellement.

Il faut interpréter cette caractéristique des partis politiques nationalistes à la fois par la qualité de leurs cadres et par celle de leur clientèle. La clientèle des partis nationalistes est une clientèle urbaine. Ces ouvriers, ces instituteurs, ces petits artisans et commerçants qui ont commencé - au rabais s'entend - à profiter de la situation coloniale ont des intérêts particuliers. Ce que cette clientèle réclame, c'est l'amélioration de son sort, l'augmentation de ses salaires. Le dialogue n'est jamais rompu entre ces partis politiques et le colonialisme. On discute d'aménagements, de représentation électorale, de liberté de la presse, de liberté d'association. On discute réformes. Aussi ne faut-il pas s'étonner de voir un grand nombre d'indigènes militer dans les succursales des formations politiques de la métropole. Ces indigènes se battent sur un mot d'ordre abstrait : «Le pouvoir au prolétariat », oubliant que, dans leur région, c'est d'abord sur des mots d'ordre nationalistes qu'il faut mener le combat. L'intellectuel colonisé a investi son agressivité dans sa volonté à peine voilée de s'assimiler au monde colonial. Il a mis son agressivité au service de ses intérêts pro- 
pres, de ses intérêts d'individu. Ainsi prend facilement naissance une sorte de classe [61] d'esclaves libérés individuellement, d'esclaves affranchis. Ce que l'intellectuel réclame, c'est la possibilité de multiplier les affranchis, la possibilité d'organiser une authentique classe d'affranchis. Les masses, par contre, n'entendent pas voir augmenter les chances de succès des individus. Ce qu'elles exigent, ce n'est pas le statut du colon, mais la place du colon. Les colonisés, dans leur immense majorité, veulent la ferme du colon. Il ne s'agit pas pour eux d'entrer en compétition avec le colon. Ils veulent sa place.

La paysannerie est laissée systématiquement de côté par la propagande de la plupart des partis nationalistes. Or il est clair que, dans les pays coloniaux, seule la paysannerie est révolutionnaire. Elle n'a rien à perdre et tout à gagner. Le paysan, le déclassé, l'affamé est l'exploité qui découvre le plus vite que la violence, seule, paie. Pour lui, il n'y a pas de compromis, pas de possibilité d'arrangement. La colonisation ou la décolonisation, c'est simplement un rapport de forces. L'exploité s'aperçoit que sa libération suppose tous les moyens et d'abord la force. Lorsqu'en 1956, après la capitulation de M. Guy Mollet devant les colons d'Algérie, le Front de libération nationale, dans un tract célèbre, constatait que le colonialisme ne lâche que le couteau sur la gorge, aucun Algérien vraiment n'a trouvé ces termes trop violents. Le tract ne faisait qu'exprimer ce que tous les Algériens ressentaient au plus profond d'eux-mêmes: le colonialisme n'est pas une machine à penser, n'est pas un corps doué de raison. Il est la violence à l'état de nature et ne peut s'incliner que devant une plus grande violence.

Au moment de l'explication décisive, la bourgeoisie colonialiste, qui était jusque-là restée coite, entre en action. Elle introduit cette nouvelle notion qui est à proprement parler une création de la situation coloniale: la non-violence. Dans sa forme brute cette non-violence signifie aux élites intellectuelles et économiques colonisées que la bour- 
geoisie colonialiste a les mêmes intérêts qu'elles et qu'il devient donc indispensable, [62] urgent, de parvenir à un accord pour le salut commun. La non-violence est une tentative de régler le problème colonial, autour d'un tapis vert avant tout geste irréversible, toute effusion de sang, tout acte regrettable. Mais si les masses, sans attendre que les chaises soient disposées autour du tapis vert, n'écoutent que leur propre voix et commencent les incendies et les attentats, on voit alors les «élites » et les dirigeants des partis bourgeois nationalistes se précipiter vers les colonialistes et leur dire: «C'est très grave ! On ne sait pas comment tout cela va finir, il faut trouver une solution, il faut trouver un compromis. »

Cette notion de compromis est très importante dans le phénomène de la décolonisation, car elle est loin d'être simple. Le compromis, en effet, concerne à la fois le système colonial et la jeune bourgeoisie nationale. Les tenants du système colonial découvrent que les masses risquent de tout détruire. Le sabotage des ponts, la destruction des fermes, les répressions, la guerre frappent durement l'économie. Compromis également pour la bourgeoisie nationale qui, ne discernant pas très bien les conséquences possibles de ce typhon, craint en réalité d'être balayée par cette formidable bourrasque et ne cesse de dire aux colons: "Nous sommes encore capables d'arrêter le carnage, les masses ont encore confiance en nous, faites vite si vous ne voulez pas tout compromettre. » Un degré de plus, et le dirigeant du parti nationaliste prend ses distances vis-à-vis de cette violence. Il affirme hautement qu'il n'a rien à faire avec ces Mau-Mau, avec ces terroristes, avec ces égorgeurs. Dans le meilleur des cas, il se cantonne dans un no man's land entre les terroristes et les colons et se présente volontiers comme « interlocuteur»: ce qui signifie que, les colons ne pouvant discuter avec les Mau-Mau, il veut bien, lui, entamer des négociations. C'est ainsi que l'arrière-garde de la lutte nationale, cette partie du peuple qui n'a jamais cessé d'être de l'autre côté de la lutte, se trouve placée par une sorte de gymnastique à l'avant-garde des négocia- 
tions et du compromis - parce que précisément elle s'est bien gardée de jamais rompre le contact avec le colonialisme.

Avant la négociation, la majorité des partis nationalistes se contentent dans le meilleur des cas d'expliquer, d'excuser cette "sauvagerie ». Ils ne revendiquent pas la lutte populaire et il n'est pas rare qu'ils se laissent aller, dans des cercles fermés, à condamner tels actes spectaculaires déclarés odieux par la presse et l'opinion de la métropole. Le souci de voir les choses objectivement constitue l'excuse légitime de cette politique d'immobilisme. Mais cette attitude classique de l'intellectuel colonisé et des dirigeants des parties nationalistes n'est pas, en réalité, objective. En fait, ils ne sont pas sûrs que cette violence impatiente des masses soit le moyen le plus efficace de défendre leurs propres intérêts. Il y a aussi qu'ils sont convaincus de l'inefficacité des méthodes violentes. Pour eux, aucun doute n'est permis, toute tentative de briser l'oppression coloniale par la force est une conduite de désespoir, une conduite-suicide. C'est que, dans leur cerveau, les tanks des colons et les avions de chasse occupent une place énorme. Quand on leur dit : il faut agir, ils voient des bombes se déverser sur leur tête, des blindés s'avancer le long des chemins, la mitraille, la police... et ils restent assis. Ils partent perdants. Leur incapacité à triompher par la violence n'a pas besoin d'être démontrée, ils l'assument dans leur vie quotidienne et dans leurs manœuvres. Ils en sont restés à la position puérile qu'Engels adoptait dans sa célèbre polémique avec cette montagne de puérilité qu'était $M$. Dühring: «De même que Robinson a pu se procurer une épée, nous pouvons tout aussi bien admettre que Vendredi apparaît un beau matin avec un revolver chargé à la main, et alors tout le rapport de "violence" se renverse: Vendredi commande et Robinson est forcé de trimer... Donc, le revolver triomphe de l'épée et même l'amateur d'axiomes le plus puéril concevra sans doute que la violence n'est pas un simple acte de volonté, mais exige pour sa mise en œuvre des conditions préalables très réelles, notamment des instruments, dont le plus parfait l'emporte sur le moins parfait: qu'en outre ces instruments doivent être produits, 
ce qui signifie aussi que le producteur d'instruments de violence plus parfaits, grossièrement parlant des armes, l'emporte sur le producteur [64] des moins parfaits et qu'en un mot la victoire de la violence repose sur la production d'armes, et celle-ci à son tour sur la production en général, donc... sur la "puissance économique", sur l'État économique, sur les moyens matériels qui sont à la disposition de la violen$\mathrm{ce}^{3}$. » De fait, les dirigeants réformistes ne disent pas autre chose: «Avec quoi voulez-vous vous battre contre les colons. Avec vos couteaux? Avec vos fusils de chasse?»

Il est vrai que les instruments sont importants dans le domaine de la violence, puisque tout repose en définitive sur la répartition de ces instruments. Mais il se trouve que, dans ce domaine, la libération des territoires coloniaux apporte un éclairage nouveau. On a vu par exemple que pendant la campagne d'Espagne, cette authentique guerre coloniale, Napoléon, malgré des effectifs qui ont atteint, pendant les offensives du printemps 1810, le chiffre énorme de 400000 hommes, fut contraint de reculer. Pourtant l'armée française faisait trembler toute l'Europe par ses instruments de guerre, par la valeur de ses soldats, par le génie militaire de ses capitaines. Face aux moyens énormes des troupes napoléoniennes, les Espagnols qu'animait une foi nationale inébranlable, découvrirent cette fameuse guérilla que, vingt-cinq ans plus tôt les miliciens américains avaient expérimentée contre les troupes anglaises. Mais la guérilla du colonisé ne serait rien comme instrument de violence opposé à d'autres instruments de violence, si elle n'était pas un élément nouveau dans le processus global de la compétition entre trusts et monopoles.

Au début de la colonisation, une colonne pouvait occuper des territoires immenses : le Congo, le Nigéria, la Côte-d'Ivoire, etc. Mais au-

3 Friedrich Engels, Anti-Dühring, 2 e partie, chapitre III, « Théorie de la violence», Éditions Sociales, p. 199. [Livre disponible dans Les Classiques des sciences sociales. JMT.] 
jourd' hui la lutte nationale du colonisé s'insère dans une situation absolument nouvelle. Le capitalisme, dans sa période d'essor, voyait dans les colonies une source de matières [65] premières qui, manufacturées, pouvaient être déversées sur le marché européen. Après une phase d'accumulation du capital, il en arrive aujourd'hui à modifier sa conception de la rentabilité d'une affaire. Les colonies sont devenues un marché. La population coloniale est une clientèle qui achète. Dès lors, si la garnison doit être éternellement renforcée, si le commerce se ralentit, c'est-à-dire si les produits manufacturés et industrialisés ne peuvent plus être exportés, c'est la preuve que la solution militaire doit être écartée. Une domination aveugle de type esclavagiste n'est économiquement pas rentable pour la métropole. La fraction monopoliste de la bourgeoisie métropolitaine ne soutient pas un gouvernement dont la politique est uniquement celle de l'épée. Ce que les industriels et les financiers de la métropole attendent de leur gouvernement, ce n'est pas qu'il décime les peuplades mais qu'il sauvegarde, à l'aide de conventions économiques, leurs « intérêts légitimes».

Il existe donc une complicité objective du capitalisme avec les forces violentes qui éclatent dans le territoire colonial. De plus, le colonisé n'est pas seul face à l'oppresseur. Il y a, bien sûr, l'aide politique et diplomatique des pays et des peuples progressistes. Mais il y a surtout la compétition, la guerre impitoyable que se livrent les groupes financiers. Une conférence de Berlin avait pu répartir l'Afrique déchiquetée entre trois ou quatre pavillons. Actuellement, ce qui est important, ce n'est pas que telle région africaine soit terre de souveraineté française ou belge: ce qui importe, c'est que les zones économiques soient protégées. Le pilonnage d'artillerie, la politique de la terre brûlée ont fait place à la sujétion économique. Aujourd'hui on ne mène plus de guerre de répression contre tel sultan rebelle. On est plus élégant, moins sanguinaire, et on décide la liquidation pacifique du régime castriste. On essaie d'étrangler la Guinée, on supprime Mossadegh. Le dirigeant national qui a peur de la violence a donc tort s'il s'imagine que le colonialisme va « tous nous massacrer ». Les militaires, bien sûr, 
continuent à jouer avec les poupées datant de la conquête, mais les milieux financiers ont vite fait de les ramener à la réalité.

[66]

C'est pourquoi il est demandé aux partis politiques nationalistes raisonnables d'exposer le plus clairement possible leurs revendications et de chercher avec le partenaire colonialiste, dans le calme et l'absence de passion, une solution qui respecte les intérêts des deux parties. On voit que ce réformisme nationaliste, qui se présente souvent comme une caricature du syndicalisme, s'il décide d'agir le fera à travers des voies hautement pacifiques: débrayages dans les quelques industries implantées dans les villes, manifestations de masse pour acclamer le leader, boycott des autobus ou des denrées importées. Toutes ces actions servent à la fois à faire pression sur le colonialisme et à permettre au peuple de se dépenser. Cette pratique de l'hibernothérapie, cette cure de sommeil du peuple peut quelquefois réussir. Alors, de la discussion autour du tapis vert, surgit la promotion politique qui permet à M. M'ba, président de la République du Gabon, de dire très solennellement à son arrivée en visite officielle à Paris : "Le Gabon est indépendant, mais entre le Gabon et la France rien n'est changé, tout continue comme avant." De fait, le seul changement c'est que $M$. M'ba est président de la République gabonaise et qu'il est reçu par le président de la République française.

La bourgeoisie colonialiste est aidée dans son travail de tranquillisation des colonisés par l'inévitable religion. Tous les saints qui ont tendu la deuxième joue, qui ont pardonné les offenses, qui ont reçu sans tressaillir les crachats et les insultes sont expliqués, donnés en exemple. Les élites des pays colonisés, ces esclaves affranchis, quand ils sont à la tête du mouvement, finissent inéluctablement par produire un ersatz de combat. Ils utilisent /'esclavage de leurs frères pour faire honte aux esclavagistes ou pour fournir un contenu idéologique d'humanitarisme falot aux groupes financiers concurrents de leurs oppresseurs. Jamais, en vérité, ils ne font appel réellement aux escla- 
ves, jamais ils ne les mobilisent concrètement. Bien au contraire, au moment de la vérité, c'est-à-dire, pour eux, du mensonge, ils brandissent la menace d'une mobilisation des [67] masses comme l'arme décisive qui provoquerait comme par enchantement la « fin du régime colonial ». Il se trouve évidemment au sein de ces partis politiques, parmi les cadres, des révolutionnaires qui tournent délibérément le dos à la farce de l'indépendance nationale. Mais rapidement leurs interventions, leurs initiatives, leurs mouvements de colère indisposent la machine du parti. Progressivement, ces éléments sont isolés, puis carrément écartés. Dans le même temps, comme s'il y avait concomitance dialectique, la police colonialiste va leur tomber dessus. Sans sécurité dans les villes, évités par les militants, rejetés par les autorités du parti, ces indésirables au regard incendiaire vont échouer dans les campagnes. C'est alors qu'ils s'aperçoivent avec une sorte de vertige que les masses paysannes comprennent à demi-mot leurs propos et, sans transition, leur posent la question dont ils n'ont pas préparé la réponse : «C'est pour quand? ?

Cette rencontre des révolutionnaires venus des villes et des campagnards retiendra plus loin notre attention. Il convient maintenant de revenir aux partis politiques, pour montrer le caractère tout de même progressiste de leur action. Dans leurs discours, les dirigeants politiques «nomment» la nation. Les revendications du colonisé reçoivent ainsi une forme. Il n'y a pas de contenu, il n'y a pas de programme politique et social. Il y a une forme vague mais néanmoins nationale, un cadre, ce que nous appelons l'exigence minimum. Les hommes politiques qui prennent la parole, qui écrivent dans les journaux nationalistes, font rêver le peuple. Ils évitent la subversion mais, en fait, introduisent de terribles ferments de subversion dans la conscience des auditeurs ou des lecteurs. Souvent on utilise la langue nationale ou tribale. C'est, là encore, entretenir le rêve, permettre à l'imagination de gambader hors de l'ordre colonial. Quelquefois encore ces hommes politiques disent: "Nous les Nègres, nous les Arabes», et cette appellation lourde d'ambivalence pendant la période coloniale reçoit une sorte 
de sacralisation. Les hommes politiques nationalistes jouent avec le feu. Car, comme [68] le confiait récemment un dirigeant africain à un groupe de jeunes intellectuels: "Réfléchissez avant de parler aux masses, elles s'enflamment vite. »Il y a donc une ruse de l'histoire, qui joue terriblement aux colonies.

Quand le dirigeant politique convie le peuple à un meeting, on peut dire qu'il y a du sang dans l'air. Pourtant le dirigeant, très souvent, se préoccupe surtout de «montrer» ses forces... pour n'avoir pas à les utiliser. Mais l'agitation ainsi entretenue - aller, venir, entendre des discours, voir le peuple réuni, les policiers autour, les démonstrations militaires, les arrestations, les déportations de leaders -, tout ce remue-ménage donne au peuple l'impression que le moment est venu, pour lui, de faire quelque chose. Dans ces moments d'instabilité, les partis politiques multiplient les appels au calme vers la gauche, tandis que, sur leur droite, ils scrutent l'horizon, tâchant de déchiffrer les intentions libérales du colonialisme.

Le peuple utilise également pour se maintenir en forme, pour entretenir sa capacité révolutionnaire, certains épisodes de la vie de la collectivité. Le bandit, par exemple, qui tient la campagne pendant des journées face aux gendarmes lancés à ses trousses, celui qui, dans un combat singulier, succombe après avoir abattu quatre ou cinq policiers, celui qui se suicide pour ne pas «donner» ses complices constituent pour le peuple des phares, des schèmes d'actions, des « héros». Et il ne sert à rien, évidemment, de dire que tel héros est un voleur, une crapule ou un dépravé. Si l'acte pour lequel cet homme est poursuivi par les autorités colonialistes est un acte exclusivement dirigé contre une personne ou un bien colonial, alors la démarcation est nette, flagrante. Le processus d'identification est automatique.

Il faut signaler également le rôle que joue, dans ce phénomène de maturation, l'histoire de la résistance nationale à la conquête. Les grandes figures du peuple colonisé sont toujours celles qui ont dirigé la résistance nationale à l'invasion. Béhanzin, Soundiata, Samory, Abdel 
Kader revivent avec une particulière intensité dans la période qui précède l'action. C'est [69] la preuve que le peuple s'apprête à se remettre en marche, à interrompre le temps mort introduit par le colonialisme, à faire l'Histoire.

Le surgissement de la nation nouvelle, la démolition des structures coloniales sont le résultat soit d'une lutte violente du peuple indépendant, soit de l'action, contraignante pour le régime colonial, de la violence périphérique assumée par d'autres peuples colonisés.

Le peuple colonisé n'est pas seul. En dépit des efforts du colonialisme, ses frontières demeurent perméables aux nouvelles, aux échos. Il découvre que la violence est atmosphérique, qu'elle éclate çà et là, et çà et là emporte le régime colonial. Cette violence qui réussit a un rôle non seulement informateur mais opératoire pour le colonisé. La grande victoire du peuple vietnamien à Dien-Bien-Phu n'est plus, à strictement parler, une victoire vietnamienne. À partir de juillet 1954 , le problème que se sont posé les peuples coloniaux a été le suivant: «Que faut-il faire pour réaliser un Dien-Bien-Phu ? Comment s'y prendre? » De la possibilité de ce Dien-Bien-Phu, aucun colonisé ne pouvait plus douter. Ce qui faisait le problème, c'était l'aménagement des forces, leur organisation, leur date d'entrée en action. Cette violence ambiante ne modifie pas les seuls colonisés mais également les colonialistes qui prennent conscience de Dien-Bien-Phu multiples. C'est pourquoi une véritable panique ordonnée va s'emparer des gouvernements colonialistes. Leur propos est de prendre les devants, de tourner à droite le mouvement de libération, de désarmer le peuple : vite, décolonisons. Décolonisons le Congo avant qu'il ne se transforme en Algérie. Votons la loi-cadre pour l'Afrique, créons la Communauté, rénovons cette Communauté mais, je vous en conjure, décolonisons, décolonisons... On décolonise à une telle allure qu'on impose l'indépendance à HouphouëtBoigny. À la stratégie du Dien-Bien-Phu, définie par le colonisé, le colonialiste répond par la stratégie de l'encadrement... dans le respect de la souveraineté des États. 
[70]

Mais revenons à cette violence atmosphérique, à cette violence à fleur de peau. Nous avons vu dans le développement de sa maturation que beaucoup de courroies la prennent en charge et la mènent à la sortie. En dépit des métamorphoses que le régime colonial lui impose dans les luttes tribales ou régionalistes, la violence s'achemine, le colonisé identifie son ennemi, met un nom sur tous ses malheurs et jette dans cette nouvelle voie toute la force exacerbée de sa haine et de sa colère. Mais comment passons-nous de l'atmosphère de violence à la violence en action? Qu'est-ce qui fait exploser la marmite? Il y a le fait, d'abord, que ce développement ne laisse pas inentamée la béatitude du colon. Le colon qui « connaît » les indigènes s'aperçoit à plusieurs indices que quelque chose est en train de changer. Les bons indigènes se font rares, les silences s'étendent à l'approche de l'oppresseur. Quelquefois les regards se font durs, les attitudes et les propos carrément agressifs. Les partis nationalistes s'agitent, multiplient les meetings et, dans le même temps, les forces de police sont augmentées, des renforts de troupe arrivent. Les colons, les agriculteurs surtout, isolés dans leurs fermes, sont les premiers à s'alarmer. Ils réclament des mesures énergiques.

Les autorités prennent en effet des mesures spectaculaires, arrêtent un ou deux leaders, organisent des défilés militaires, des manœuvres, des vols aériens. Les démonstrations, les exercices belliqueux, cette odeur de poudre qui, maintenant, charge l'atmosphère, ne font pas reculer le peuple. Ces baïonnettes et ces canonnades renforcent son agressivité. Une atmosphère de drame s'installe, où chacun veut prouver qu'il est prêt à tout. C'est dans ces circonstances que le coup part tout seul car les nerfs sont fragilisés, la peur s'est installée, on est sensible de la gâchette. Un incident banal et le mitraillage commence : c'est Sétif en Algérie, ce sont les Carrières centrales au Maroc, c'est Moramanga à Madagascar. 
Les répressions, loin de briser l'élan, scandent les progrès de la conscience nationale. Aux colonies, les hécatombes, à partir [71] d'un certain stade de développement embryonnaire de la conscience, renforcent cette conscience, car elles indiquent qu'entre oppresseurs et opprimés tout se résout par la force. Il faut signaler ici que les partis politiques n'ont pas lancé le mot d'ordre de l'insurrection armée, n'ont pas préparé cette insurrection. Toutes ces répressions, tous ces actes suscités par la peur ne sont pas voulus par les dirigeants. Les événements les prennent de court. C'est alors que le colonialisme peut décider d'arrêter les leaders nationalistes. Mais aujourd'hui les gouvernements des pays colonialistes savent parfaitement qu'il est très dangereux de priver les masses de leur leader. Car alors le peuple, n'étant plus bridé, se jette dans la jacquerie, les mutineries et les «meurtres bestiaux». Les masses donnent libre cours à leurs « instincts sanguinaires » et imposent au colonialisme la libération des leaders, auxquels reviendra la tâche difficile de ramener le calme. Le peuple colonisé, qui avait spontanément investi sa violence dans la tâche colossale de destruction du système colonial, va se retrouver en peu de temps avec le mot d'ordre inerte, infécond: "Libérez $X$ ou $Y{ }^{4}$. " Alors le colonialisme libérera ces hommes et discutera avec eux. L'heure des bals populaires a commencé.

Dans un autre cas, l'appareil des partis politiques peut rester intact. Mais à la suite de la répression colonialiste et de la réaction spontanée du peuple les partis se trouvent débordés par leurs militants. La violence des masses s'oppose vigoureusement aux forces militaires de l'occupant, la situation se détériore et pourrit. Les dirigeants en liberté restent alors sur la touche. Devenus soudain inutiles avec leur bureaucratie et leur programme raisonnable, on les voit, loin des événements, tenter la suprême imposture de «parler au nom de la nation muselée ». En règle générale, le colonialisme se jette avec avidité sur cette aubaine, transforme ces inutiles en interlocuteurs et, en

4 Il peut arriver que le leader soit l'expression authentique des masses colonisées. Dans ce cas, le colonialisme va profiter de sa détention pour essayer de lancer de nouveaux leaders. 
quatre [72] secondes, leur donne l'indépendance, à charge pour eux de ramener l'ordre.

On voit donc que tout le monde est conscient de cette violence et que la question n'est pas toujours d'y répondre par une plus grande violence mais plutôt de voir comment désamorcer la crise.

Qu'est-ce donc en réalité que cette violence? Nous l'avons vu, c'est l'intuition qu'ont les masses colonisées que leur libération doit se faire, et ne peut se faire que par la force. Par quelle aberration de l'esprit ces hommes sans technique, affamés et affaiblis, non rompus aux méthodes d'organisation, en arrivent-ils, face à la puissance économique et militaire de l'occupant, à croire que seule la violence pourra les libérer? Comment peuvent-ils espérer triompher?

Car la violence, et c'est là le scandale, peut constituer, en tant que méthode, le mot d'ordre d'un parti politique. Des cadres peuvent appeler le peuple à la lutte armée. Il faut réfléchir à cette problématique de la violence. Que le militarisme allemand décide de régler ses problèmes de frontières par la force ne nous surprend point, mais que le peuple angolais, par exemple, décide de prendre les armes, que le peuple algérien rejette toute méthode qui ne soit pas violente, prouve que quelque chose s'est passé ou est en train de se passer. Les hommes colonisés, ces esclaves des temps modernes, sont impatients. Ils savent que seule cette folie peut les soustraire à l'oppression coloniale. Un nouveau type de rapports s'est établi dans le monde. Les peuples sous-développés font craquer leur chaîne et l'extraordinaire, c'est qu'ils réussissent. On peut prétendre qu'à l'heure du spoutnik il est ridicule de mourir de faim, mais pour les masses colonisées l'explication est moins lunaire. La vérité, c'est qu'aucun pays colonialiste n'est aujourd'hui capable d'adopter la seule forme de lutte qui aurait une chance de réussir: l'implantation prolongée de forces d'occupation importantes.

Sur le plan intérieur, les pays colonialistes se trouvent confrontés à des contradictions, à des revendications ouvrières qui exigent l'emploi de leurs forces policières. De plus, dans la [73] conjoncture interna- 
tionale actuelle, ces pays ont besoin de leurs troupes pour protéger leur régime. Enfin l'on connaît le mythe des mouvements de libération dirigés de Moscou. Dans l'argumentation paniquarde du régime, cela signifie: «Si cela continue, les communistes risquent de profiter de ces troubles pour s'infiltrer dans ces régions. »

Dans l'impatience du colonisé, le fait qu'il brandisse à bout de bras la menace de la violence prouve qu'il est conscient du caractère exceptionnel de la situation contemporaine et qu'il entend en profiter. Mais aussi sur le plan de l'expérience immédiate, le colonisé, qui a l'occasion de voir le monde moderne pénétrer jusque dans les coins les plus reculés de la brousse, prend une conscience très aiguë de ce qu'il ne possède pas. Les masses, par une sorte de raisonnement... infantile, se convainquent que toutes ces choses leur ont été volées. C'est pourquoi dans certains pays sous-développés les masses vont très vite et comprennent, deux ou trois ans après l'indépendance, qu'elles ont été frustrées, que « ça ne valait pas la peine » de se battre si ça ne devait pas vraiment changer. En 1789, après la Révolution bourgeoise, les plus petits paysans français ont profité substantiellement de ce bouleversement. Mais il est banal de constater et de dire que dans la majorité des cas, pour $95 \%$ de la population des pays sous-développés, l'indépendance n'apporte pas de changement immédiat. L'observateur averti se rend compte de l'existence d'une sorte de mécontentement larvé, comme ces braises qui, après l'extinction d'un incendie, menacent toujours de s'enflammer.

On dit alors que les colonisés veulent aller trop vite. Or, ne l'oublions jamais, il n'y a pas bien longtemps on affirmait leur lenteur, leur paresse, leur fatalisme. On aperçoit déjà que la violence dans les voies bien précises au moment de la lutte de libération ne s'éteint pas magiquement après la cérémonie des couleurs nationales. Elle s'éteint d'autant moins que la construction nationale continue à s'inscrire dans le cadre de la compétition décisive du capitalisme et du socialisme. 
Cette compétition donne une dimension quasi universelle aux revendications les plus localisées. Chaque meeting, chaque acte de répression retentit dans l'arène internationale. Les meurtres de Sharpeville ont secoué l'opinion pendant des mois. Dans les journaux, sur les antennes, dans les conversations privées, Sharpeville est devenu un symbole. C'est à travers Sharpeville que des hommes et des femmes ont abordé le problème de l'apartheid en Afrique du Sud. Et l'on ne peut prétendre que seule la démagogie explique le soudain intérêt des Grands pour les petites affaires des régions sous-développées. Chaque jacquerie, chaque sédition dans le tiers monde s'insère dans le cadre de la guerre froide. Deux hommes sont matraqués à Salisbury, et voici que l'ensemble d'un bloc se met en branle, parle de ces deux hommes et, à l'occasion de ce matraquage, soulève le problème particulier de la Rhodésie - le reliant à l'ensemble de l'Afrique et à la totalité des hommes colonisés. Mais l'autre bloc, également, mesure, à l'ampleur de la campagne menée, les faiblesses locales de son système. Les peuples colonisés se rendent compte qu'aucun clan ne se désintéresse des incidents locaux. Ils cessent de se limiter à leurs horizons régionaux, saisis qu'ils sont dans cette atmosphère de secousse universelle.

Lorsque, tous les trois mois, on apprend que la $6^{e}$ ou la $7^{e}$ flotte fait mouvement vers telle côte, lorsque Khrouchtchev menace de sauver Castro à coups de fusées, lorsque Kennedy, à propos du Laos, décide d'envisager les solutions extrêmes, le colonisé ou le nouvel indépendant a l'impression que, bon gré, mal gré, il est entraîné dans une sorte de marche effrénée. En fait, il marche déjà. Prenons, par exemple, le cas des gouvernements de pays récemment libérés. Les hommes au pouvoir passent les deux tiers de leur temps à surveiller les alentours, à prévenir le danger qui les menace, et l'autre tiers à travailler pour le pays. En même temps, ils se cherchent des appuis. Obéissant à la même dialectique, les oppositions nationales se détournent avec mépris des voies parlementaires. Elles cherchent [75] des alliés qui acceptent de les soutenir dans leur entreprise brutale de sédition. L'atmosphère de violence, après avoir imprégné la phase coloniale, continue de dominer 
la vie nationale. Car, nous l'avons dit, le tiers monde n'est pas exclu. Bien au contraire, il est au centre de la tourmente. C'est pourquoi, dans leurs discours, les hommes d'État des pays sous-développés maintiennent indéfiniment le ton d'agressivité et d'exaspération qui aurait dû normalement disparaître. L'on comprend également l'impolitesse si souvent signalée des nouveaux dirigeants. Mais ce que l'on voit moins, c'est l'extrême courtoisie de ces mêmes dirigeants dans leurs contacts avec les frères ou les camarades. L'impolitesse est d'abord une conduite avec les autres, avec les anciens colonialistes qui viennent voir et enquêter. L'ex-colonisé a trop souvent l'impression que la conclusion de ces enquêtes est déjà rédigée. Le déplacement du journaliste est une justification. Les photographies qui illustrent l'article apportent la preuve qu'on sait de quoi l'on parle, qu'on y est allé. L'enquête se propose de vérifier l'évidence: tout va mal là-bas depuis que nous n'y sommes plus. Les journalistes se plaignent souvent d'être mal reçus, de ne pas travailler dans de bonnes conditions, de trouver un mur d'indifférence ou d'hostilité. Tout cela est normal. Les dirigeants nationalistes savent que l'opinion internationale est forgée uniquement par la presse occidentale. Or, quand un journaliste occidental nous interroge, c'est rarement pour nous rendre service. Dans la guerre d'Algérie, par exemple, les reporters français les plus libéraux n'ont pas cessé d'utiliser des épithètes ambiguës pour caractériser notre lutte. Quand on leur en fait le reproche, ils répondent en toute bonne foi qu'ils sont objectifs. Pour le colonisé, l'objectivité est toujours dirigée contre lui. On comprend également ce nouveau ton qui a submergé la diplomatie internationale à l'Assemblée générale des Nations unies en septembre 1960. Les représentants des pays coloniaux étaient agressifs, violents, outranciers, mais les peuples coloniaux n'ont pas trouvé qu'ils exagéraient. Le radicalisme des porte-parole africains a provoqué le mûrissement de l'abcès et a permis de mieux voir le [76] caractère inadmissible des veto, du dialogue des Grands, et surtout le rôle infime réservé au tiers monde.

La diplomatie telle qu'elle a été inaugurée par les peuples nouvellement indépendants n'est plus en nuances, en sous-entendus, en passes 
magnétiques. C'est que ces porte-parole sont chargés par leurs peuples de défendre à la fois l'unité de la nation, le progrès des masses vers le bien-être et le droit des peuples à la liberté et au pain.

C'est donc une diplomatie en mouvement, en furie, qui contraste étrangement avec le monde immobile, pétrifié, de la colonisation. Et quand $M$. Khrouchtchev brandit son soulier à l'ONU et en martèle la table, aucun colonisé, aucun représentant des pays sous-développés ne rit. Car ce que M. Khrouchtchev montre aux pays colonisés qui le regardent c'est que lui, le moujik, qui par ailleurs possède des fusées, traite ces misérables capitalistes comme ils le méritent. De même, Castro siégeant en tenue militaire à l'ONU ne scandalise pas les pays sous-développés. Ce que montre Castro, c'est la conscience qu'il a de l'existence du régime continué de la violence. L'étonnant, c'est qu'il ne soit pas entré à l'ONU avec sa mitraillette: mais peut-être s'y seraiton opposé ? Les jacqueries, les actes désespérés, les groupes armés de coutelas ou de haches trouvent leur nationalité dans la lutte implacable qui dresse l'un contre l'autre le capitalisme et le socialisme.

En 1945, les 45000 morts de Sétifs pouvaient passer inaperçus: en 1947, les 90000 morts de Madagascar pouvaient faire l'objet d'un simple entrefilet dans les journaux; en 1952, les 200000 victimes de la répression au Kenya pouvaient rencontrer une indifférence relative. C'est que les contradictions internationales n'étaient pas suffisamment tranchées. Déjà la guerre de Corée et la guerre d'Indochine avaient inauguré une nouvelle phase. Mais c'est surtout Budapest et Suez qui constituent les moments décisifs de cette confrontation.

Forts du soutien inconditionnel des pays socialistes, les colonisés se lancent avec les armes qu'ils ont contre la citadelle [77] inexpugnable du colonialisme. Si cette citadelle est invulnérable aux couteaux et aux poings nus, elle ne l'est plus quand on décide de tenir compte du contexte de la guerre froide.

Dans cette conjoncture nouvelle, les Américains prennent très au sérieux leur rôle de patron du capitalisme international. Dans un pre- 
mier temps, ils conseillent aux pays européens de décoloniser à l'amiable. Dans un deuxième temps, ils n'hésitent pas à proclamer d'abord le respect puis le soutien du principe : l'Afrique aux Africains. Les États-Unis ne craignent pas aujourd'hui de dire officiellement qu'ils sont les défenseurs du droit des peuples à disposer d'eux-mêmes. Le dernier voyage de M. Mennen-Williams n'est que l'illustration de la conscience qu'ont les Américains que le tiers monde ne doit pas être sacrifié. On comprend dès lors pourquoi la violence du colonisé n'est désespérée que si on la compare in abstracto à la machine militaire des oppresseurs. Par contre, si on la situe dans la dynamique internationale, on s'aperçoit qu'elle constitue une terrible menace pour l'oppresseur. La persistance des jacqueries et de l'agitation Mau-Mau déséquilibre la vie économique de la colonie mais ne met pas en danger la métropole. Ce qui est plus important aux yeux de l'impérialisme, c'est la possibilité pour la propagande socialiste de s'infiltrer dans les masses, de les contaminer. C'est déjà un grave danger dans la période froide du conflit; mais que deviendrait, en cas de guerre chaude, cette colonie, pourrie par les guérillas meurtrières?

Le capitalisme se rend compte alors que sa stratégie militaire a tout à perdre au développement des guerres nationales. Aussi, dans le cadre de la coexistence pacifique, toutes les colonies sont-elles appelées à disparaître et, à l'extrême, le neutralisme à être respecté par le capitalisme. Ce qu'il faut éviter avant tout, c'est l'insécurité stratégique, l'ouverture des masses sur une doctrine ennemie, la haine radicale de dizaines de millions d'hommes. Les peuples colonisées sont parfaitement conscients de ces impératifs qui dominent la vie politique internationale. [78] C'est pourquoi même ceux qui tonnent contre la violence décident et agissent toujours en fonction de cette violence planétaire. Aujourd'hui la coexistence pacifique entre les deux blocs entretient et provoque la violence dans les pays coloniaux. Demain, peutêtre verrons-nous se déplacer ce domaine de la violence après la libération intégrale des territoires coloniaux. Peut-être verrons-nous se poser la question des minorités. Déjà certaines d'entre elles n'hésitent 
pas à prôner des méthodes violentes pour résoudre leurs problèmes et ce n'est pas par hasard si, nous dit-on, des extrémistes nègres aux États-Unis forment des milices et s'arment en conséquence. Ce n'est pas par hasard non plus si, dans le monde dit libre, il y a des comités de défense des minorités juives en URSS et si le général de Gaulle, dans l'un de ses discours, a versé quelques larmes sur les millions de musulmans opprimés par la dictature communiste. Le capitalisme et l'impérialisme sont convaincus que la lutte contre le racisme et les mouvements de libération nationale sont purement et simplement des troubles télécommandés, fomentés de «l'extérieur». Aussi décidentils d'utiliser cette tactique efficace: Radio-Europe libre, comité de soutien des minorités dominées... Ils font de l'anticolonialisme, comme les colonels français en Algérie faisaient de la guerre subversive avec les SAS ou les services psychologiques. Ils «utilisaient le peuple contre le peuple ». On sait ce que cela donne.

Cette atmosphère de violence, de menace, ces fusées brandies n'effraient pas et ne désorientent pas les colonisés. Nous avons vu que toute leur histoire récente les dispose à « comprendre » cette situation. Entre la violence coloniale et la violence pacifique dans laquelle baigne le monde contemporain il y a une sorte de correspondance complice, une homogénéité. Les colonisés sont adaptés à cette atmosphère. Ils sont, pour une fois, de leur temps. On s'étonne quelquefois que les colonisés, plutôt que d'offrir une robe à leur femme, achètent un poste à transistors. On ne devrait pas. Les colonisés sont persuadés que leur destin se joue maintenant. Ils vivent dans une atmosphère [79] de fin du monde et ils estiment que rien ne doit leur échapper. C'est pourquoi ils comprennent très bien Phouma et Phoumi, Lumumba et Tschombé, Ahidjo et Moumié, Kenyatta et ceux qu'on jette périodiquement en avant pour le remplacer. Ils comprennent très bien tous ces hommes car ils démasquent les forces qui sont derrière eux. Le colonisé, l'homme sous-développé sont aujourd'hui des animaux politiques au sens le plus planétaire du terme. 
L'indépendance a certes apporté aux hommes colonisés la réparation morale et consacré leur dignité. Mais ils n'ont pas encore eu le temps d'élaborer une société, de construire et d'affirmer des valeurs. Le foyer incandescent où le citoyen et l'homme se développent et s'enrichissent dans des domaines de plus en plus larges n'existe pas encore. Placés dans une sorte d'indétermination, ces hommes se persuadent assez facilement que tout va se décider ailleurs, pour tout le monde, en même temps. Quant aux dirigeants, face à cette conjoncture, ils hésitent et choisissent le neutralisme.

Il y aurait beaucoup à dire sur le neutralisme. Certains l'assimilent à une sorte de mercantilisme infect qui consisterait à prendre à droite et à gauche. Or, le neutralisme, cette création de la guerre froide s'il permet aux pays sous-développés de recevoir l'aide économique des deux parties, ne permet pas, en fait, à chacune de ces deux parties de venir en aide comme il le faudrait aux régions sous-développées. Ces sommes littéralement astronomiques qui sont investies dans les recherches militaires, ces ingénieurs transformés en techniciens de la guerre nucléaire pourraient, en quinze ans, augmenter le niveau de vie des pays sous-développés de $60 \%$. On voit donc que l'intérêt bien compris des pays sous-développés ne réside ni dans la prolongation ni dans l'accentuation de cette guerre froide. Mais il se trouve qu'on ne leur demande pas leur avis. Alors, quand ils en ont la possibilité, ils se désengagent. Mais le peuvent-ils réellement? Voici, par exemple, que la France expérimente en Afrique ses [80] bombes atomiques. Si l'on excepte les motions, les meetings et les ruptures diplomatiques fracassantes, on ne peut pas dire que les peuples africains aient pesé, dans ce secteur précis, sur l'attitude de la France.

Le neutralisme produit chez le citoyen du tiers monde une attitude d'esprit qui se traduit dans la vie courante par une intrépidité et une fierté hiératique qui ressemblent étrangement au défi. Ce refus affirmé du compromis, cette volonté toute dure de ne pas s'attacher 
rappellent le comportement de ces adolescents fiers et dépouillés, toujours prêts à se sacrifier pour un mot. Tout cela désarçonne les observateurs occidentaux. Car il y a à proprement parler un scandale entre ce que ces hommes prétendent être et ce qu'ils ont derrière eux. Ce pays sans tramways, sans troupes, sans argent ne justifie pas la bravade qu'ils étalent au grand jour. C'est, à n'en pas douter, de l'imposture. Le tiers monde donne souvent l'impression qu'il jubile dans le drame et qu'il lui faut sa dose hebdomadaire de crises. Ces leaders de pays vides, qui parlent fort, irritent. On a envie de les faire taire. Or, on les courtise. On leur offre des fleurs. On les invite. Disons-le, on se les arrache. Cela, c'est du neutralisme. Illettrés à $98 \%$, il existe cependant à leur propos une littérature colossale. Ils voyagent énormément. Les dirigeants des pays sous-développés, les étudiants des pays sous-développés sont des clients dorés pour les compagnies aériennes. Les responsables africains et asiens ont la possibilité, dans le même mois, de suivre un enseignement sur la planification socialiste à Moscou et sur les bienfaits de l'économie libérale à Londres ou à Columbia University. Les syndicalistes africains, pour leur part, progressent à une cadence accélérée. À peine leur confie-t-on des postes dans les organismes de direction qu'ils décident de se constituer en centrales autonomes. Ils n'ont pas ces cinquante ans de pratique syndicale passés dans le cadre d'un pays industrialisé, mais ils savent déjà que le syndicalisme apolitique est un non-sens. Ils n'ont pas affronté la machine bourgeoise, ils n'ont pas [81] développé leur conscience dans la lutte des classes, mais peut-être n'est-ce pas nécessaire. Peut-être. Nous verrons que cette volonté totalisante, qui se caricature souvent en globalisme, est l'une des caractéristiques les plus fondamentales des pays sous-développés.

Mais revenons au combat singulier du colonisé et du colon. Il s'agit, on le voit, de la lutte armée franche. Les exemples historiques sont: I'Indochine, l'Indonésie, et, bien sûr, l'Afrique du Nord. Mais ce qu'il ne faut pas perdre de vue, c'est qu'elle aurait pu éclater n'importe où, en Guinée comme en Somalie, et encore aujourd'hui elle peut éclater partout où le colonialisme entend encore durer, en Angola par exemple. 
L'existence de la lutte armée indique que le peuple décide de ne faire confiance qu'aux moyens violents. Lui à qui on n'a jamais cessé de dire qu'il ne comprenait que le langage de la force, décide de s'exprimer par la force. En fait, depuis toujours, le colon lui a signifié le chemin qui devait être le sien, s'il voulait se libérer. L'argument que choisit le colonisé lui a été indiqué par le colon et, par un ironique retour des choses, c'est le colonisé qui, maintenant, affirme que le colonialiste ne comprend que la force. Le régime colonial tire sa légitimité de la force et à aucun moment n'essaie de ruser avec cette nature des choses. Chaque statue, celle de Faidherbe ou de Lyautey, de Bugeaud ou du sergent Blandan, tous ces conquistadors juchés sur le sol colonial n'arrêtent pas de signifier une seule et même chose: "Nous sommes ici par la force des baïonnettes... » On complète aisément. Pendant la phase insurrectionnelle, chaque colon raisonne à partir d'une arithmétique précise. Cette logique n'étonne pas les autres colons mais il est important de dire qu'elle n'étonne pas non plus les colonisés. Et d'abord, l'affirmation de principe: «C'est eux ou nous 》 ne constitue pas un paradoxe, puisque le colonialisme, avons-nous vu, est justement l'organisation d'un monde manichéiste, d'un monde compartimenté. E† quand, préconisant des moyens précis, le colon demande à chaque représentant de la minorité qui opprime de descendre 30 ou 100 ou 200 indigènes, il s'aperçoit que personne n'est indigné et qu'à [82] l'extrême tout le problème et de savoir si on peut faire ça d'un seul coup ou par étapes 5 .

Ce raisonnement qui prévoit très arithmétiquement la disparition du peuple colonisé ne bouleverse pas le colonisé d'indignation morale. Il a toujours su que ses rencontres avec le colon se dérouleraient dans un

5 Il est évident que ce nettoyage par le vide détruit la chose qu' on voulait sauver. $C$ 'est bien ce que signale Sartre quand il dit : «En somme par le fait même de les répéter (il s'agit des idées racistes) on révèle que l'union simultanée de tous contre les indigènes est irréalisable, qu'elle n'est que récurrence tournante et que d'ailleurs cette union ne pourrait se faire comme groupement actif que pour massacrer les colonisés, tentation perpétuelle et absurde du colon, qui revient, si elle était d'ailleurs réalisable, à supprimer d'un coup la colonisation. » Critique de la raison dialectique, p. 346. 
champ clos. Aussi le colonisé ne perd-il pas son temps en lamentations et ne cherche-t-il presque jamais à ce qu'on lui rende justice dans le cadre colonial. En fait, si l'argumentation du colon trouve le colonisé inébranlable, c'est que ce dernier a pratiquement posé le problème de sa libération en des termes identiques: "Constituons-nous en groupes de deux cents ou de cinq cents et que chaque groupe s'occupe d'un colon. » C'est dans cette disposition d'esprit réciproque que chacun des protagonistes commence la lutte.

Pour le colonisé, cette violence représente la praxis absolue. Aussi le militant est-il celui qui travaille. Les questions posées au militant par l'organisation portent la marque de cette vision des choses: «Où astu travaillé ? Avec qui ? Qu'as-tu fait? » Le groupe exige que chaque individu réalise un acte irréversible. En Algérie, par exemple, où la presque totalité des hommes qui ont appelé le peuple à la lutte nationale étaient condamnés à mort ou recherchés Par la police française, la confiance était proportionnelle au caractère désespéré de chaque cas. Un nouveau militant était sûr quand il ne pouvait plus rentrer dans le système colonial. Ce mécanisme aurait, paraît-il, existé au Kenya chez les Mau-Mau qui exigeaient que chaque membre du [83] groupe frappât la victime. Chacun était donc personnellement responsable de la mort de cette victime. Travailler, c'est travailler à la mort du colon. La violence assumée permet à la fois aux égarés et aux proscrits du groupe de revenir, de retrouver leur place, de réintégrer. La violence est ainsi comprise comme la médiation royale. L'homme colonisé se libère dans et par la violence. Cette praxis illumine l'agent parce qu'elle lui indique les moyens et la fin. La poésie de Césaire prend dans la perspective précise de la violence une signification prophétique. Il est bon de rappeler l'une des pages les plus décisives de sa tragédie où le Rebelle (tiens!) s'explique : 


\section{LE REBELLE (dur)}

Mon nom : offensé : mon prénom : humilié : mon état : révolté : mon âge : l'âge de la pierre.

\section{LA MÈRE}

Ma race : la race humaine. Ma religion : la fraternité...

\section{LE REBELLE}

Ma race; la race tombée. Ma religion... mais ce n'est pas vous qui la préparerez avec votre désarmement... c'est moi avec ma révolte et mes pauvres poings serrés et ma tête hirsute.

(Très calme.)

Je me souviens d'un jour de novembre; il n'avait pas six mois et le maître est entré dans la case fuligineuse comme une lune rousse, et il tâtait ses petits membres musclés, c'était un très bon maître, il promenait d'une caresse ses doigts gros sur son petit visage plein de fossettes. Ses yeux bleus riaient et sa bouche le taquinait de choses sucrées : ce sera une bonne pièce, dit-il en me regardant, et il disait d'autres choses aimables, le maître, qu'il fallait s'y prendre très tôt, que ce n'était pas trop de vingt ans pour faire un bon chrétien et un bon esclave, bon sujet et bien dévoué, un bon garde-chiourme de commandeur, [84] oeil vif et le bras ferme. Et cet homme spéculait sur le berceau de mon fils un berceau de garde-chiourme. Nous rampâmes coutelas au poing...

\section{LA MÈRE}

Hélas tu mourras. 


\title{
LE REBELLE
}

Tué... je l'ai tué de mes propres mains... Oui : de mort féconde et plantureuse... c'était la nuit. Nous rampâmes parmi les cannes à sucre. Les coutelas riaient aux étoiles, mais on se moquait des étoiles. Les cannes à sucre nous balafraient le visage de ruisseaux de lames vertes.

\section{LA MÈRE}

J'avais rêvé d'un fils pour fermer les yeux de sa mère.

\section{LE REBELLE}

J'ai choisi d'ouvrir sur un autre soleil les yeux de mon fils.

\author{
LA MÈRE \\ ...O mon fils... de mort mauvaise et pernicieuse.
}

\section{LE REBELLE}

Mère, de mort vivace et somptueuse.

\section{LA MĖRE}

pour avoir trop hai

\section{LE REBELLE}

pour avoir trop aimé. 


\section{LA MĖRE}

Épargne-moi, j'étouffe de tes liens. Je saigne de tes blessures.

\section{LE REBELLE}

Et le monde ne m'épargne pas... Il n'y a pas dans le monde un pauvre type lynché, un pauvre homme torturé, en qui je ne sois assassiné et humilié.

[85]

\section{LA MÈRE}

Dieu du ciel, délivre-le.

\section{LE REBELLE}

Mon cœur tu ne me délivreras pas de mes souvenirs... C'était un soir de novembre...

Et subitement des clameurs éclairèrent le silence,

Nous avions bondi, nous, les esclaves; nous, le fumier: nous, les bêtes au sabot de patience.

Nous courions comme des forcenés : les coups de feu éclatèrent... Nous frappions. La sueur et le sang nous faisaient une fraîcheur. Nous frappions parmi les cris et les cris devinrent plus stridents et une grande clameur s'éleva vers l'est, c'étaient les communs qui brûlaient et la flamme flaqua douce sur nos joues.

Alors ce fut l'assaut donné à la maison du maître.

On tirait des fenêtres.

Nous forçâmes les portes. 
La chambre du maître était grande ouverte. La chambre du maître était brillamment éclairée, et le maître était là très calme... et les nôtres s'arrêtèrent... c'était le maître... J'entrai. C'est toi, me dit-il, très calme... C'était moi, c'était bien moi, lui disais-je, le bon esclave, le fidèle esclave, l'esclave esclave, et soudain ses yeux furent deux ravets apeurés les jours de pluie... je frappai, le sang gicla: c'est le seul baptême dont je me souvienne aujourd'hui 6 .

On comprend que dans cette atmosphère la quotidienneté devienne tout simplement impossible. On ne peut plus être fellah, souteneur ou alcoolique comme avant. La violence du régime colonial et la contreviolence du colonisé s'équilibrent et se répondent dans une homogénéité réciproque extraordinaire. Ce règne de la violence sera d'autant plus terrible que le peuplement métropolitain sera important. Le développement de la [86] violence au sein du peuple colonisé sera proportionnel à la violence exercée par le régime colonial contesté. Les gouvernements métropolitains sont dans la première phase de cette période insurrectionnelle, esclaves des colons. Ces colons menacent à la fois les colonisés et leurs gouvernements. Ils utiliseront contre les uns et les autres les mêmes méthodes. L'assassinat du maire d'Évian, dans son mécanisme et ses motivations, s'identifie à l'assassinat d'Ali Boumendjel. Pour les colons, l'alternative n'est pas entre une Algérie algérienne et une Algérie française mais entre une Algérie indépendante et une Algérie coloniale. Tout le reste est littérature ou tentative de trahison. La logique du colon est implacable et l'on n'est désarçonné par la contre-logique déchiffrée dans la conduite du colonisé que dans la mesure où l'on n'a pas préalablement mis au jour les mécanismes de pensée du colon. Dès lors que le colonisé choisit la contre-violence, les représailles policières appellent mécaniquement les représailles des forces nationales. Il n'y a pas cependant équivalence des résultats, car

6 Aimé Césaire, Les Armes miraculeuses (Et les chiens se taisaient), Gallimard, p. 133 à 137. 
les mitraillages par avion ou les canonnades de la flotte dépassent en horreur et en importance les réponses du colonisé. Ce va-et-vient de la terreur démystifie définitivement les plus aliénés des colonisés. Ils constatent en effet sur le terrain que tous les discours sur l'égalité de la personne humaine entassés les uns sur les autres ne masquent pas cette banalité qui veut que les sept Français tués ou blessés au col de Sakamody soulèvent l'indignation des consciences civilisées tandis que « comptent pour du beurre » la mise à sac des douars Guergour, de la dechra Djerah, le massacre des populations qui avaient précisément motivé l'embuscade. Terreur, contre-terreur, violence, contreviolence... Voilà ce qu'enregistrent dans l'amertume les observateurs quand ils décrivent le cercle de la haine, si manifeste et si tenace en Algérie.

Dans les luttes armées, il y a ce qu'on pourrait appeler le point de non-retour. C'est presque toujours la répression énorme englobant tous les secteurs du peuple colonisé qui le réalise. Ce point fut atteint en Algérie en 1955 avec les 12000 victimes de [87] Philippeville et en 1956 avec l'installation par Lacoste des milices urbaines et rurales 7 .

7 Il faut revenir à cette période pour mesurer l'importance de cette décision du pouvoir français en Algérie. Ainsi, dans le $n^{\circ} 4$ du 28.3.1957 de Résistance algérienne, on peut lire: «Répondant au vœu de l'Assemblée générale des Nations unies, le Gouvernement français vient de décider en Algérie la création de milices urbaines. Assez de sang versé, avait dit l'ONU, Lacoste répond: Constituons des milices. Cessez-le-feu, conseillait l'ONU, Lacoste vocifère: Armons les civils. Les deux parties en présence sont invitées à entrer en contact pour s'entendre sur une solution démocratique et pacifique recommandait l'ONU, Lacoste décrète que dorénavant tout Européen sera armé et devra tirer sur quiconque lui paraîtra suspect. La répression sauvage, inique, confinant au génocide devra avant toutes choses être combattue par les autorités, estimait-on alors. Lacoste répond: Systématisons la répression, organisons la chasse aux Algériens. Et symboliquement il remet les pouvoirs civils aux militaires, les pouvoirs militaires aux civils. Le cercle est fermé. Au milieu l'Algérien, désarmé, affamé, traqué, bousculé, frappé, lynché, bientôt abattu parce que suspect. Aujourd'hui, en Algérie, il n'y a pas un Français qui ne soit autorisé, invité à faire usage de son arme. Pas un Français, en Algérie, un mois après l'appel au calme de l'ONU, qui n'ait la permission, l'obligation de découvrir, de susciter, de poursuivre des suspects.

«Un mois après le vote de la motion finale de l'Assemblée générale des $\mathrm{Na}$ tions unies, pas un Européen, en Algérie, à être étranger à la plus épouvantable 
entreprise d'extermination des temps modernes. Solution démocratique? D'accord concède Lacoste, commençons par supprimer les Algériens. Pour cela, armons les civils et laissons faire. La presse parisienne dans son ensemble, a accueilli avec réserve la création de ces groupes armés. Milice fascistes, a-t-on dit. Oui. Mais, à l'échelle de l'individu et du droit des gens, qu'est le fascisme sinon le colonialisme au sein de pays traditionnellement colonialistes? Assassinats systématiquement légalisés, recommandés, a-t-on avancé. Mais la chair algérienne ne porte-t-elle pas depuis cent trente ans des blessures de plus en plus ouvertes, de plus en plus nombreuses, de plus en plus radicales? Attention, conseille $M$. Kenne-Vignes, parlementaire MRP, ne risque-t-on pas, en créant ces milices, de voir se creuser bientôt un abîme entre les deux communautés d'Algérie? Oui. Mais le statut colonial n'est-ce pas l'asservissement organisé de tout un peuple? La Révolution algérienne est précisément la contestation affirmée de cet asservissement et de cet abîme. La Révolution algérienne s'adresse à la nation occupante et lui dit: "Enlevez vos crocs de la chair algérienne meurtrie et blessée ! Donnez voix au peuple algérien!»

«La création de ces milices, dit-on, permettra d'alléger les tâches de l'Armée. Elle libérera des unités dont la mission sera de protéger les frontières tunisienne et marocaine. Une armée forte de six cent mille hommes. La quasitotalité de la Marine et de l'Aviation. Une police énorme, expéditive, au palmarès ahurissant, ayant absorbé les ex-tortionnaires des peuples tunisien et marocain. Des unités territoriales fortes de cent mille hommes. Il faut alléger l'Armée. Créons des milices urbaines. Tant il est vrai que la frénésie hystérique et criminelle de Lacoste en impose, même aux Français clairvoyants. La vérité est que la création de ces milices porte dans sa justification sa propre contradiction. Les tâches de l'Armée française sont infinies. Dès lors qu'on lui fixe comme objectif la remise du bâillon à la bouche algérienne se ferme toujours la porte sur l'avenir. Surtout, on s'interdit d'analyser, de comprendre, de mesurer la profondeur et la densité de la Révolution algérienne: chefs d'arrondissements, chefs dîlots, chefs de rues, chefs de buildings, chefs d'étages... Au quadrillage en surface s'ajoute aujourd' hui le quadrillage en hauteur.

«En 48 heures deux mille candidatures sont enregistrées. Les Européens d'Algérie ont immédiatement répondu à l'appel au meurtre de Lacoste. Désormais, chaque Européen devra recenser dans son secteur les Algériens survivants. Renseignements, « réponse rapide » au terrorisme, détection de suspects, liquidation de « fuyards», renforcement des services de police. Certainement, il faut alléger les tâches de l'Armée. Au ratissage en surface s'ajoute aujourd'hui le ratissage en hauteur. Au meurtre artisanal s'ajoute aujourd'hui le meurtre planifié. Arrêtez l'écoulement de sang, avait conseillé l'ONU. Le meilleur moyen d'y parvenir, réplique Lacoste, est qu'il n'y ait plus de sang à verser. Le peuple algérien après avoir été livré aux hordes de Massu est confié aux bons soins des milices urbaines. En décidant la création de ces milices, Lacoste signifie nettement qu'il ne laissera pas toucher à SA guerre. Il prouve qu'il existe un infini dans le pour- 
Alors il devint clair pour tout le [88] monde et même pour les colons que "ça ne pouvait plus recommencer» comme avant. Toutefois, le peuple colonisé ne tient pas de comptabilité. Il enregistre les vides énormes faits dans ses rangs comme une sorte de mal nécessaire. Puisque aussi bien il a décidé de répondre par la violence, il en admet toutes les conséquences. Seulement il exige qu'on ne lui demande pas non plus de tenir de comptabilité pour les autres. À la formule [89] "Tous les indigènes sont pareils», le colonisé répond: «Tous les colons sont pareils ${ }^{8}$. " Le colonisé, quand on le torture, qu'on lui tue sa femme ou qu'on la viole, ne va se plaindre à personne. Le gouvernement qui opprime pourra bien nommer chaque jour des commissions d'enquête et d'information. Aux yeux du colonisé, ces commissions n'existent pas. Et, de fait, bientôt sept ans de crimes en Algérie et pas un Français qui ait été traduit devant une cour de justice française pour le meurtre d'un Algérien. En Indochine, à Madagascar, aux colonies, l'indigène a toujours su qu'il n'y avait rien à attendre de l'autre bord. Le travail du colon est de rendre impossibles jusqu'aux rêves de liberté du colonisé. Le travail du colonisé est d'imaginer toutes les combinaisons éventuelles pour anéantir le colon. Sur le plan du raisonnement, le manichéisme du colon produit un manichéisme du colonisé. À

rissement. Certes, le voici maintenant prisonnier, mais quelle jouissance de perdre tout le monde avec soi.

"Le peuple algérien après chacune de ces décisions augmente la contracture de ses muscles et l'intensité de sa lutte. Le peuple algérien, après chacun de ces assassinats sollicités et organisés, structure davantage sa prise de conscience et solidifie sa résistance. Oui. Les tâches de l'Armée française sont infinies. Car l'unité du peuple algérien est, ô combien, infinie !! »

8 C'est pourquoi, au début des hostilités, il n'y a pas de prisonniers. C'est seulement par la politisation des cadres que les dirigeants arrivent à faire admettre aux masses : 1) que les gens qui viennent de la métropole ne sont pas toujours volontaires et quelquefois même sont écœurés par cette guerre ; 2) que l'intérêt actuel de la lutte veut que le mouvement manifeste dans son action le respect de certaines conventions internationales: 3) qu'une armée qui fait des prisonniers est une armée, et cesse d'être considérée comme un groupe d'écumeurs de routes ; 4) qu'en tout état de cause la possession des prisonniers constitue un moyen de pression non négligeable pour protéger nos militants détenus par l'ennemi. 
la théorie de «l'indigène mal absolu » répond la théorie du « colon mal absolu. »

L'apparition du colon a signifié syncrétiquement mort de la société autochtone, léthargie culturelle, pétrification des individus. Pour le colonisé, la vie ne peut surgir que du cadavre en décomposition du colon. Telle est donc cette correspondance terme à terme des deux raisonnements.

Mais il se trouve que pour le peuple colonisé cette violence, parce qu'elle constitue son seul travail, revêt des caractères positifs, [90] formateurs. Cette praxis violente est totalisante, puisque chacun se fait maillon violent de la grande chaîne, du grand organisme violent surgi comme réaction à la violence première du colonialiste. Les groupes se reconnaissent entre eux et la nation future est déjà indivise. La lutte armée mobilise le peuple, c'est-à-dire qu'elle le jette dans une seule direction, à sens unique.

La mobilisation des masses, quand elle se réalise à l'occasion de la guerre de libération, introduit dans chaque conscience la notion de cause commune, de destin national, d'histoire collective. Aussi la deuxième phase, celle de la construction de la nation, se trouve-t-elle facilitée par l'existence de ce mortier travaillé dans le sang et la colère. On comprend mieux alors l'originalité du vocabulaire utilisé dans les pays sous-développés. Pendant la période coloniale, on conviait le peuple à lutter contre l'oppression. Après la libération nationale, on le convie à lutter contre la misère, l'analphabétisme, le sousdéveloppement. La lutte, affirme-t-on, continue. Le peuple vérifie que la vie est un combat interminable.

La violence du colonisé, avons-nous dit, unifie le peuple. De par sa structure en effet, le colonialisme est séparatiste et régionaliste. Le colonialisme ne se contente pas de constater l'existence de tribus, il les renforce, les différencie. Le système colonial alimente les chefferies et réactive les vieilles confréries maraboutiques. La violence dans sa pratique est totalisante, nationale. De ce fait, elle comporte dans son intimité la liquidation du régionalisme et du tribalisme. Aussi les 
partis nationalistes se montrent-ils particulièrement impitoyables avec les caïds et les chefs coutumiers. La liquidation des caïds et des chefs est un préalable à l'unification du peuple.

Au niveau des individus, la violence désintoxique. Elle débarrasse le colonisé de son complexe d'infériorité, de ses attitudes contemplatives ou désespérées. Elle le rend intrépide, le réhabilite à ses propres yeux. Même si la lutte armée a été symbolique et même s'il est démobilisé par une décolonisation rapide, le peuple a le temps de se convaincre que la libération a été l'affaire de tous et de chacun, que le leader n'a pas de mérite [91] spécial. La violence hisse le peuple à la hauteur du leader. D'où cette espèce de réticence agressive à l'égard de la machine protocolaire que de jeunes gouvernements se dépêchent de mettre en place. Quand elles ont participé, dans la violence, à la libération nationale, les masses ne permettent à personne de se présenter en « libérateur ». Elles se montrent jalouses du résultat de leur action et se gardent de remettre à un dieu vivant leur avenir, leur destin, le sort de la patrie. Totalement irresponsables hier, elles entendent aujourd'hui tout comprendre et décider de tout. Illuminée par la violence, la conscience du peuple se rebelle contre toute pacification. Les démagogues, les opportunités, les magiciens ont désormais la tâche difficile. La praxis qui les a jetées dans un corps à corps désespéré confère aux masses un goût vorace du concret. L'entreprise de mystification devient, à long terme, pratiquement impossible. 
[93]

\author{
LES DAMNÉS DE LA TERRE \\ I \\ De la violence \\ dans le contexte international
}

\title{
Retour à la table des matières
}

Nous avons maintes fois signalé dans les pages qui précèdent que dans les régions sous-développées le responsable politique est toujours en train d'appeler son peuple au combat. Combat contre le colonialisme, combat contre la misère et le sous-développement, combat contre les traditions stérilisantes. Le vocabulaire qu'il utilise dans ses appels est un vocabulaire de chef d'État-Major: «mobilisation des masses», «front de l'agriculture », «front de l'analphabétisme», « défaites subies», «victoires remportées». La jeune nation indépendante évolue pendant les premières années dans une atmosphère de champ de bataille. C'est que le dirigeant politique d'un pays sous-développé mesure avec effroi le chemin immense que doit franchir son pays. Il en appelle au peuple et lui dit : « Ceignons-nous les reins et travaillons. » Le pays, tenacement saisi par une sorte de folie créatrice, se jette dans un effort gigantesque et disproportionné. Le programme est non seulement de s'en sortir, mais de rattraper les autres nations avec les 
moyens du bord. Si les peuples européens, pense-t-on, sont parvenus à ce stade de développement, c'est à la suite de leurs efforts. Prouvons donc au monde et à nous-mêmes que nous sommes capables des mêmes réalisations. Cette façon de poser le problème de l'évolution des pays sous-développés ne nous paraît ni juste ni raisonnable.

Les États européens ont fait leur unité nationale à un moment où les bourgeoisies nationales avaient concentré dans leurs mains la plupart des richesses. Commerçants et artisans, clercs et banquiers monopolisaient dans le cadre national les [94] finances, le commerce et les sciences. La bourgeoisie représentait la classe la plus dynamique, la plus prospère. Son accession au pouvoir lui permettait de se lancer dans des opérations décisives: industrialisation, développement des communications et bientôt recherches de débouchés « outre-mer ».

En Europe, si l'on excepte quelques nuances (I'Angleterre par exemple avait pris une certaine avance) les différents États au moment où se réalisait leur unité nationale connaissaient une situation économique à peu près uniforme. Aucune nation vraiment, par les caractères de son développement et de son évolution, n'insultait les autres.

Aujourd'hui, l'indépendance nationale, la formation nationale dans les régions sous-développées revêtent des aspects totalement nouveaux. Dans ces régions, quelques réalisations spectaculaires exceptées, les différents pays présentent la même absence d'infrastructure. Les masses luttent contre la même misère, se débattent avec les mêmes gestes et dessinent avec leurs estomacs rapetissés ce que l'on a pu appeler la géographie de la faim. Monde sousdéveloppé, monde de misère et inhumain. Mais aussi monde sans médecins, sans ingénieurs, sans administrateurs. Face à ce monde, les nations européennes se vautrent dans l'opulence la plus ostentatoire. Cette opulence européenne est littéralement scandaleuse car elle a été bâtie sur le dos des esclaves, elle s'est nourrie du sang des esclaves, elle vient en droite ligne du sol et du sous-sol de ce monde sous- 
développé. Le bien-être et le progrès de l'Europe ont été bâtis avec la sueur et les cadavres des nègres, des Arabes, des Indiens et des Jaunes. Cela, nous décidons de ne plus l'oublier. Lorsqu'un pays colonialiste, gêné par les revendications à l'indépendance d'une colonie, proclame à l'intention des dirigeants nationalistes: «Si vous voulez l'indépendance, prenez-la et retournez au Moyen Âge», le peuple nouvellement indépendant a tendance à acquiescer et à relever le défi. Et I'on voit effectivement le colonialisme retirer ses capitaux et ses techniciens et mettre en place autour du jeune État un dispositif de pression [95] économique ${ }^{9}$. L'apothéose de l'indépendance se transforme en malédiction de l'indépendance. La puissance coloniale par des moyens énormes de coercition condamne à la régression la jeune nation. En clair, la puissance coloniale dit: «Puisque vous voulez l'indépendance, prenez-la et crevez. » Les dirigeants nationalistes n'ont alors d'autre ressource que de se tourner vers leur peuple et de

9 Dans le contexte international actuel, le capitalisme n'exerce pas le blocus économique contre les seules colonies africaines ou asiatiques. Les États-Unis, avec l'opération anticastriste, inaugurent dans l'hémisphère américain un nouveau chapitre de l'histoire de la libération laborieuse de l'homme. L'Amérique latine formée de pays indépendants siégeant à l'ONU et battant monnaie devrait constituer une leçon pour l'Afrique. Ces anciennes colonies depuis leur libération subissent dans la terreur et le dénuement la loi d'airain du capitalisme occidental.

La libération de l'Afrique, le développement de la conscience des hommes ont permis aux peuples latino-américains de briser avec la vieille danse des dictatures où les régimes se succédaient en se ressemblant. Castro prend le pouvoir à Cuba et le donne au peuple. Cette hérésie est ressentie comme fléau national chez les yankees et les États-Unis organisent des brigades contrerévolutionnaires, fabriquent un gouvernement provisoire, incendient les récoltes de canne, décident enfin d'étrangler impitoyablement le peuple cubain. Mais ce sera difficile. Le peuple cubain souffrira mais il vaincra. Le président brésilien Janos Quadros, dans une déclaration d'importance historique, vient d'affirmer que son pays défendra par tous les moyens la Révolution cubaine. Les États-Unis eux aussi vont peut-être reculer devant la volonté des peuples. Ce jour-là, nous pavoiserons, car ce sera un jour décisif pour les hommes et pour les femmes du monde entier. Le dollar qui, somme toute, n'est garanti que par les esclaves répartis sur le globe, dans les puits de pétrole du Moyen-Orient, les mines du Pérou ou du Congo, les plantations de l'United Fruit ou de Firestone, cessera alors de dominer de toute sa puissance ces esclaves qui l'ont créé et qui continuent tête vide et ventre vide à le nourrir de leur substance. 
lui demander un effort grandiose. De ces hommes affamés on exige un régime d'austérité, à ces muscles atrophiés on demande un travail disproportionné. Un régime autarcique est institué et chaque État, avec les moyens misérables dont il dispose, tâche de répondre à la grande faim nationale, à la grande misère nationale. On assiste à la mobilisation d'un peuple qui dès lors s'éreinte et s'épuise face à l'Europe repue et méprisante.

[96]

D'autres pays du tiers monde refusent cette épreuve et acceptent d'en passer par les conditions de l'ancienne puissance tutélaire. Utilisant leur position stratégique, position qui les privilégie dans la lutte des blocs, ces pays concluent des accords, s'engagent. L'ancien pays dominé se transforme en pays économiquement dépendant. L'expuissance coloniale qui a maintenu intacts, et quelquefois renforcé, des circuits commerciaux de type colonialiste accepte par petites injections d'alimenter le budget de la nation indépendante. On voit donc que l'accession à l'indépendance des pays coloniaux place le monde devant un problème capital : la libération nationale des pays colonisés dévoile et rend plus insupportable leur état réel. La confrontation fondamentale qui semblait être celle du colonialisme et de l'anticolonialisme, voire du capitalisme et du socialisme, perd déjà de son importance. Ce qui compte aujourd'hui, le problème qui barre l'horizon c'est la nécessité d'une redistribution des richesses. L'humanité, sous peine d'en être ébranlée, devra répondre à cette question.

On a pu penser généralement que l'heure était venue pour le monde, et singulièrement pour le tiers monde, de choisir entre le système capitaliste et le système socialiste. Les pays sous-développés, qui ont utilisé la compétition féroce qui existe entre les deux systèmes pour assurer le triomphe de leur lutte de libération nationale, doivent cependant refuser de s'installer dans cette compétition. Le tiers monde ne doit pas se contenter de se définir par rapport à des valeurs qui l'ont précédé. Les pays sous-développés au contraire doivent 
s'efforcer de mettre au jour des valeurs qui leur soient propres, des méthodes, un style qui leur soient spécifiques. Le problème concret devant lequel nous nous trouvons n'est pas celui du choix coûte que coûte entre le socialisme et le capitalisme tels qu'ils ont été définis par des hommes de continents et d'époques différents. Nous savons, certes, que le régime capitaliste ne peut pas en tant que mode de vie nous permettre de réaliser notre tâche nationale et universelle. L'exploitation capitaliste, les trusts et les monopoles sont [97] les ennemis des pays sous-développés. Par contre le choix d'un régime socialiste, d'un régime tout entier tourné vers l'ensemble du peuple, basé sur le principe que l'homme est le bien le plus précieux, nous permettra d'aller plus vite, plus harmonieusement, rendant de ce fait impossible cette caricature de société où quelques-uns détiennent l'ensemble des pouvoirs économiques et politiques au mépris de la totalité nationale.

Mais pour que ce régime puisse valablement fonctionner, que nous puissions à tout instant respecter les principes dont nous nous inspirons, il nous faut autre chose que l'investissement humain. Certains pays sous-développés déploient dans cette direction un effort colossal. Hommes et femmes, jeunes et vieux, dans l'enthousiasme, s'engagent dans un véritable travail forcé et se proclament esclaves de la nation. Le don de soi, le mépris de toute préoccupation qui ne soit pas collective font exister une morale nationale qui réconforte l'homme, lui redonne confiance dans le destin du monde et désarme les observateurs les plus réticents. Nous croyons cependant qu'un tel effort ne pourra se poursuivre longtemps à ce rythme infernal. Ces jeunes pays ont accepté de relever le défi après le retrait inconditionnel de l'ex-pays colonial. Le pays se retrouve entre les mains de la nouvelle équipe mais en réalité il faut tout reprendre, tout repenser. Le système colonial en effet s'intéressait à certaines richesses, à certaines ressources, précisément celles qui alimentaient ses industries. Aucun bilan sérieux n'avait été fait jusqu'à présent du sol ou du sous-sol. Aussi la jeune nation indépendante se voit-elle obligée de continuer les circuits économiques mis en place par le régime colonial. Elle peut, bien sûr, exporter vers d'autres pays, vers d'autres zones monétaires mais 
la base de ses exportations n'est pas fondamentalement modifiée. Le régime colonial a cristallisé des circuits et on est contraint sous peine de catastrophe de les maintenir. Il faudrait peut-être tout recommencer, changer la nature des exportations et non pas seulement leur destination, réinterroger le sol, le sous-sol, les rivières et pourquoi pas le soleil. Or, pour ce faire il faut autre chose que l'investissement [98] humain. Il faut des capitaux, des techniciens, des ingénieurs, des mécaniciens, etc. Disons-le, nous croyons que l'effort colossal auquel sont conviés les peuples sous-développés par leurs dirigeants ne donnera pas les résultats escomptés. Si les conditions de travail ne sont pas modifiées il faudra des siècles pour humaniser ce monde rendu animal par les forces impérialistes 10.

La vérité c'est que nous ne devons pas accepter ces conditions. Nous devons carrément refuser la situation à laquelle veulent nous condamner les pays occidentaux. Le colonialisme et l'impérialisme ne sont pas quittes avec nous quand ils ont retiré de nos territoires leurs drapeaux et leurs forces de police. Pendant des siècles les capitalistes se sont comportés dans le monde sous-développé comme de véritables criminels de guerre. Les déportations, les massacres, le travail forcé, l'esclavagisme ont été les principaux moyens utilisés par le capitalisme pour augmenter ses réserves d'or et de diamants, ses richesses et pour établir sa puissance. Il y a peu de temps, le nazisme a transformé la totalité de l'Europe en véritable colonie. Les gouvernements des différentes nations européennes ont exigé des réparations et demandé la restitution en argent et en nature des richesses qui leur avaient été volées: œuvres culturelles, tableaux, sculptures, vitraux ont été rendus à leurs propriétaires. Dans la bouche des Européens au lendemain de 1945 une seule phrase: «L'Allemagne paiera.» De son côté M. Adenauer, au moment où s'ouvrait le procès Eichamnn, $a$, au nom du

10 Certains pays favorisés par un peuplement européen important accèdent à l'indépendance avec des murs et des avenues et ont tendance à oublier l'arrièrepays misérable et affamé. Ironie du sort, par une sorte de silence complice, ils font comme si leurs villes étaient contemporaines de l'indépendance. 
peuple allemand, encore une fois demandé pardon au peuple juif. $M$. Adenauer a renouvelé l'engagement de son pays à continuer de payer à l'État d'Israël les [99] sommes énormes qui doivent servir de compensation aux crimes nazis ${ }^{11}$.

Pareillement nous disons que les États impérialistes commettraient une grave erreur et une injustice inqualifiable s'ils se contentaient de retirer de notre sol les cohortes militaires, les services administratifs et d'intendance dont c'était la fonction de découvrir des richesses, de les extraire et de les expédier vers les métropoles. La réparation morale de l'indépendance nationale ne nous aveugle pas, ne nous nourrit pas. La richesse des pays impérialistes est aussi notre richesse. Sur le plan de l'universel, cette affirmation, on s'en doute, ne veut absolument pas signifier que nous nous sentons concernés par les créations de la technique ou des arts occidentaux. Très concrètement l'Europe s'est enflée de façon démesurée de l'or et des matières premières des pays coloniaux: Amérique latine, Chine, Afrique. De tous ces continents, en face desquels l'Europe aujourd'hui dresse sa tour opulente, partent depuis des siècles en direction de cette même Europe les diamants et le pétrole, la soie et le coton, les bois et les produits exoti-

11 Et il est vrai que l'Allemagne n'a pas intégralement réparé les crimes de guerre. Les indemnités imposées à la nation vaincue n'ont pas été réclamées en totalité car les nations lésées ont inclus l'Allemagne dans leur système défensif, anticommuniste. C'est cette préoccupation permanente qui anime les pays colonialistes quand ils essaient d'obtenir de leurs anciennes colonies, à défaut de l'inclusion dans le système occidental, des bases militaires et des enclaves. Ils ont décidé d'un commun accord d'oublier leurs revendications au nom de la stratégie de I'OTAN, au nom du monde libre. Et l'on a vu l'Allemagne recevoir par vagues successives des dollars et des machines. Une Allemagne redressée, forte et puissante était une nécessité pour le camp occidental. L'intérêt bien compris de l'Europe dite libre voulait une Allemagne prospère, reconstruite et capable de servir de premier rempart aux éventuelles hordes rouges. L'Allemagne a merveilleusement utilisé la crise européenne. Aussi les États-Unis et les autres États européens éprouvent-ils une légitime amertume devant cette Allemagne, hier à genou, qui leur livre aujourd'hui sur le marché économique une concurrence implacable. 
ques. L'Europe est littéralement la création du tiers monde. Les richesses qui l'étouffent [100] sont celles qui ont été volées aux peuples sous-développés. Les ports de la Hollande, Liverpool, les docks de Bordeaux et de Liverpool spécialisés dans la traite des nègres doivent leur renommée aux millions d'esclaves déportés. Et quand nous entendons un chef d'État européen déclarer la main sur le cœur qu'il lui faut venir en aide aux malheureux peuples sous-développés, nous ne tremblons pas de reconnaissance. Bien au contraire nous nous disons « c'est une juste réparation qui va nous être faite ». Aussi n'accepterons-nous pas que l'aide aux pays sous-développés soit un programme de « sœurs de charité ». Cette aide doit être la consécration d'une double prise de conscience, prise de conscience par les colonisés que cela leur est dît et par les puissances capitalistes qu'effectivement elles doivent payer ${ }^{12}$. Que si, par inintelligence - ne parlons pas d'ingratitude -, les pays capitalistes refusaient de payer, alors la dialectique implacable de leur propre système se chargerait de les asphyxier. Les jeunes nations, c'est un fait, attirent peu les capitaux privés. De multiples raisons légitiment et expliquent cette réserve des monopoles. Dès que les capitalistes savent, et ils sont évidemment les premiers à le savoir, que leur gouvernement s'apprête à décoloniser, ils se dépêchent de retirer de la colonie la totalité de leurs capitaux. La fuite spectaculaire des capitaux est l'un des phénomènes les plus constants de la décolonisation.

Les compagnies privées, pour investir dans les pays indépendants, exigent des conditions qui se révèlent à l'expérience [101] inacceptables ou irréalisables. Fidèles au principe de rentabilité immédiate qui

12 «Distinguer radicalement l'édification du socialisme en Europe des "rapports avec le tiers monde" (comme si nous n'avions avec celui-ci que des relations d'extériorité), c'est, consciemment ou non, donner le pas à l'aménagement de I'héritage colonial sur la libération des pays sous-développés, c'est vouloir construire un socialisme de luxe sur les fruits de la rapine impériale - comme, à l'intérieur d'un gang, on se répartirait plus ou moins équitablement le butin, quitte à en distribuer un peu aux pauvres sous forme de bonnes œuvres, en oubliant que c'est à eux qu'on l'a volé ». Marcel Péju, "Mourir pour de Gaulle?», Les Temps modernes, $n^{\circ}$ 175-176, octobre-novembre 1960. 
est le leur dès qu'ils vont "outre-mer», les capitalistes se montrent réticents à l'égard de tout investissement à long terme. Ils sont rebelles et souvent ouvertement hostiles aux prétendus programmes de planification des jeunes équipes au pouvoir. À la rigueur ils accepteraient volontiers de prêter de l'argent aux jeunes États mais à la condition que cet argent serve à acheter des produits manufacturés, des machines, donc à faire tourner les usines de la métropole.

En fait, la méfiance des groupes financiers occidentaux s'explique par leur souci de ne prendre aucun risque. Aussi exigent-ils une stabilité politique et un climat social serein qu'il est impossible d'obtenir si l'on tient compte de la situation lamentable de la population globale au lendemain de l'indépendance. Alors, à la recherche de cette garantie que ne peut assurer l'ancienne colonie, ils exigent le maintien de certaines garnisons ou l'entrée du jeune État dans des pactes économiques ou militaires. Les compagnies privées font pression sur leur propre gouvernement pour qu'au moins les bases militaires soient installées dans ces pays avec pour mission d'assurer la protection de leurs intérêts. En dernier ressort ces compagnies demandent à leur gouvernement de garantir les investissements qu'elles décident de faire dans telle ou telle région sous-développée.

Il se trouve que peu de pays réalisent les conditions exigées par les trusts et les monopoles. Aussi les capitaux, privés de débouchés sûrs, restent-ils bloqués en Europe et s'immobilisent. Ils s'immobilisent d'autant plus que les capitalistes se refusent à investir sur leur propre territoire. La rentabilité dans ce cas est en effet dérisoire et le contrôle fiscal désespère les plus audacieux.

La situation est à long terme catastrophique. Les capitaux ne circulent plus ou voient leur circulation considérablement diminuée. Les banques suisses refusent les capitaux, l'Europe étouffe. Malgré les sommes énormes englouties dans les dépenses militaires le capitalisme international est aux abois.

Mais un autre danger le menace. Dans la mesure en effet où le tiers monde est abandonné et condamné à la régression, en [102] tout cas à 
la stagnation, par l'égoïsme et l'immoralité des nations occidentales, les peuples sous-développés décideront d'évoluer en autarcie collective. Les industries occidentales seront rapidement privées de leurs débouchés outre-mer. Les machines s'amoncelleront dans les entrepôts et, sur le marché européen, se déroulera une lutte inexorable entre les groupes financiers et les trusts. Fermeture d'usines, débauchage et chômage amèneront le prolétariat européen à déclencher une lutte ouverte contre le régime capitaliste. Les monopoles s'apercevront alors que leur intérêt bien compris est d'aider et d'aider massivement et sans trop de conditions les pays sous-développés. On voit donc que les jeunes nations du tiers monde ont tort de faire risette aux pays capitalistes. Nous sommes puissants de notre bon droit et de la justesse de nos positions. Nous devons au contraire dire et expliquer aux pays capitalistes que le problème fondamental de l'époque contemporaine n'est pas la guerre entre le régime socialiste et eux. Il faut mettre fin à cette guerre froide qui ne mène nulle part, arrêter la préparation de la nucléarisation du monde, investir généreusement et aider techniquement les régions sous-développées. Le sort du monde dépend de la réponse qui sera donnée à cette question.

Et que les régimes capitalistes n'essaient pas d'intéresser les régimes socialistes au « sort de l'Europe » face aux multitudes colorées et affamées. L'exploit du commandant Gagarine, n'en déplaise au général de Gaulle, n'est pas une réussite qui fait « honneur à l'Europe ». Depuis quelque temps les chefs d'État des régimes capitalistes, les hommes de culture ont à l'égard de l'Union soviétique une attitude ambivalente. Après avoir coalisé toutes leurs forces pour anéantir le régime socialiste, ils comprennent maintenant qu'il faut compter avec lui. Alors ils se font aimables, multiplient les manœuvres de séduction et rappellent constamment au peuple soviétique qu'il « appartient à l'Europe ».

En agitant le tiers monde comme une marée qui menacerait d'engloutir toute l'Europe, on n'arrivera pas à diviser les forces [103] progressistes qui entendent conduire l'humanité vers le bonheur. Le tiers monde n'entend pas organiser une immense croisade de la faim 
contre toute l'Europe. Ce qu'il attend de ceux qui l'ont maintenu en esclavage pendant des siècles, c'est qu'ils l'aident à réhabiliter l'homme, à faire triompher l'homme partout, une fois pour toutes.

Mais il est clair que nous ne poussons pas la naïveté jusqu'à croire que cela se fera avec la coopération et la bonne volonté des gouvernements européens. Ce travail colossal qui consiste à réintroduire l'homme dans le monde, l'homme total, se fera avec l'aide décisive des masses européennes qui, il faut qu'elles le reconnaissent, se sont souvent ralliées sur les problèmes coloniaux aux positions de nos maîtres communs. Pour cela, il faudrait d'abord que les masses européennes décident de se réveiller, secouent leurs cerveaux et cessent de jouer au jeu irresponsable de la Belle au bois dormant. 
[105]

\section{LES DAMNÉS DE LA TERRE}

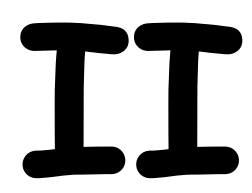

\section{GRANDEUR ET FAIBLESSES DE LA SPONTANÉITÉ}


[107]

Les réflexions sur la violence nous ont amené à prendre conscience de l'existence fréquente d'un décalage, d'une différence de rythme entre les cadres du parti nationaliste et les masses. Dans toute organisation politique ou syndicale il existe classiquement un fossé entre les masses qui exigent l'amélioration immédiate et totale de leur situation et les cadres qui, mesurant les difficultés susceptibles d'être créées par le patronat, limitent et restreignent leurs revendications. C'est pourquoi on constate souvent un mécontentement tenace des masses vis-à-vis des cadres. Après chaque journée de revendication, alors que les cadres célèbrent la victoire, les masses ont bel et bien l'impression d'avoir été trahies. C'est la multiplication des manifestations revendicatives, la multiplication des conflits syndicaux qui provoqueront la politisation de ces masses. Un syndicaliste politisé étant celui qui sait qu'un conflit local n'est pas une explication décisive entre lui et le patronat. Les intellectuels colonisés qui ont étudié dans leurs métropoles respectives le fonctionnement des partis politiques mettent en place de semblables formations afin de mobiliser les masses et de faire pression sur l'administration coloniale. La naissance de partis nationalistes dans les pays colonisés est contemporaine de la constitution d'une élite intellectuelle et commerçante. Les élites vont attacher une importance fondamentale à l'organisation en tant que telle et le fétichisme de l'organisation prendra souvent le pas sur l'étude rationnelle de la société coloniale. La notion de parti est une notion importée de la métropole. Cet instrument des luttes modernes est plaqué tel quel sur une réalité protéiforme, [108] déséquilibrée, où coexistent à 
la fois l'esclavagisme, le servage, le troc, l'artisanat et les opérations boursières.

La faiblesse des partis politiques ne réside pas seulement dans l'utilisation mécanique d'une organisation qui conduit la lutte du prolétariat au sein d'une société capitaliste hautement industrialisée. Sur le plan limité du type d'organisation, des innovations, des adaptations auraient dû voir le jour. La grande erreur, le vice congénital de la majorité des partis politiques dans les régions sous-développées a été, selon le schéma classique, de s'adresser en priorité aux éléments les plus conscients : le prolétariat des villes, les artisans et les fonctionnaires, c'est-à-dire une infime partie de la population qui ne représente guère plus de un pour cent.

Or si ce prolétariat comprenait la propagande du parti et lisait sa littérature il était beaucoup moins préparé à répondre aux éventuels mots d'ordre de lutte implacable pour la libération nationale. On l'a maintes fois signalé : dans les territoires coloniaux, le prolétariat est le noyau du peuple colonisé le plus choyé par le régime colonial. Le prolétariat embryonnaire des villes est relativement privilégié. Dans les pays capitalistes, le prolétariat n'a rien à perdre, il est celui qui, éventuellement, aurait tout à gagner. Dans les pays colonisés le prolétariat a tout à perdre. Il représente en effet la fraction du peuple colonisé nécessaire et irremplaçable pour la bonne marche de la machine coloniale: conducteurs de tramways, de taxis, mineurs, dockers, interprètes, infirmiers, etc. Ce sont ces éléments qui constituent la clientèle la plus fidèle des partis nationalistes et qui par la place privilégiée qu'ils occupent dans le système colonial constituent la fraction «bourgeoise » du peuple colonisé.

Aussi comprend-on que la clientèle des partis politiques nationalistes soit avant tout urbaine: agents de maîtrise, ouvriers, intellectuels et commerçants résidant essentiellement dans les villes. Leur type de pensée porte déjà en de nombreux points la marque du milieu technique et relativement aisé dans lequel ils évoluent. Ici le « modernisme » 
est roi. Ce sont ces [109] mêmes milieux qui vont lutter contre les traditions obscurantistes, qui vont réformer les coutumes, entrant ainsi en lutte ouverte contre le vieux socle de granit qui constitue le fonds national.

Les partis nationalistes, dans leur immense majorité, éprouvent une grande méfiance à l'égard des masses rurales. Ces masses leur donnent en effet l'impression de s'enliser dans l'inertie et dans l'infécondité. Assez rapidement les membres des partis nationalistes (ouvriers des villes et intellectuels) en arrivent à porter sur les campagnes le même jugement péjoratif que les colons. Mais si l'on tâche de comprendre les raisons de cette méfiance des partis politiques envers les masses rurales il faut retenir le fait que le colonialisme a souvent renforcé ou assis sa domination en organisant la pétrification des campagnes. Encadrées par les marabouts, les sorciers et les chefs coutumiers, les masses rurales vivent encore au stade féodal, la toute-puissance de cette structure moyenâgeuse étant alimentée par les agents administratifs ou militaires colonialistes.

La jeune bourgeoisie nationale, commerçante surtout, va entrer en compétition avec ces seigneurs féodaux dans des secteurs multiples: marabouts et sorciers qui barrent la route aux malades qui pourraient consulter le médecin, djemaas qui jugent, rendant inutiles les avocats, caïds qui utilisent leur puissance politique et administrative pour lancer un commerce ou une ligne de transports, chefs coutumiers s'opposant au nom de la religion et de la tradition à l'introduction de négoces et de produits nouveaux.

La jeune classe de commerçants et de négociants colonisés a besoin de la disparition de ces prohibitions et de ces barrières pour se développer. La clientèle indigène qui représente la chasse gardée des féodaux et qui se voit plus ou moins interdire l'achat de produits nouveaux constitue donc un marché que l'on se dispute.

Les cadres féodaux forment un écran entre les jeunes nationalistes occidentalisés et les masses. Chaque fois que les élites [110] font un 
effort en direction des masses rurales, les chefs de tribus, les chefs de confréries, les autorités traditionnelles multiplient les mises en garde, les menaces, les excommunications. Ces autorités traditionnelles qui ont été confirmées par la puissance occupante voient sans plaisir se développer les tentatives d'infiltration des élites dans les campagnes. Elles savent que les idées susceptibles d'être introduites par ces éléments venus des villes contestent le principe même de la pérennité des féodalités. Aussi leur ennemi n'est-il point la puissance occupante avec laquelle elles font, somme toute, bon ménage mais ces modernistes qui entendent désarticuler la société autochtone et par là même leur enlever le pain de la bouche.

Les éléments occidentalisés éprouvent à l'égard des masses paysannes des sentiments qui rappellent ceux que l'on trouve au sein du prolétariat des pays industrialisés. L'histoire des révolutions bourgeoises et l'histoire des révolutions prolétariennes ont montré que les masses paysannes constituent souvent un frein à la révolution. Les masses paysannes dans les pays industrialisés sont généralement les éléments les moins conscients, les moins organisés et aussi les plus anarchistes. Elles présentent tout un ensemble de traits, individualisme, indiscipline, amour du gain, aptitude aux grandes colères et aux profonds découragements, définissant un comportement objectivement réactionnaire.

Nous avons vu que les partis nationalistes calquent leurs méthodes et leurs doctrines sur les partis occidentaux, aussi, dans la majorité des cas, n'orientent-ils leur propagande en direction de ces masses. En réalité l'analyse rationnelle de la société colonisée, si elle avait été pratiquée, leur aurait montré que les paysans colonisés vivent dans un milieu traditionnel dont les structures sont demeurées intactes, alors que dans les pays industrialisés c'est ce milieu traditionnel qui a été lézardé par les progrès de l'industrialisation. C'est au sein du prolétariat embryonnaire qu'on trouve aux colonies des comportements individualistes. En abandonnant les campagnes où la démographie pose des problèmes insolubles, les paysans sans terre, qui [111] constituent le 
lumpen-prolétariat, se ruent vers les villes, s'entassent dans les bidonvilles et tâchent de s'infiltrer dans les ports et les cités nés de la domination coloniale. Les masses paysannes, elles, continuent de vivre dans un cadre immobile et les bouches en surnombre n'ont d'autre ressource que d'émigrer vers les cités. Le paysan qui reste sur place défend avec ténacité ses traditions et, dans la société colonisée, représente l'élément discipliné dont la structure sociale demeure communautaire. Il est vrai que cette vie immobile, crispée sur des cadres rigides, peut donner naissance épisodiquement à des mouvements à base de fanatisme religieux, à des guerres tribales. Mais dans leur spontanéité les masses rurales demeurent disciplinées, altruistes. L'individu s'efface devant la communauté.

Les paysans ont une méfiance à l'égard de l'homme de la ville. Habillé comme l'Européen, parlant sa langue, travaillant avec lui, habitant parfois dans son quartier, il est considéré par les paysans comme un transfuge qui a abandonné tout ce qui constitue le patrimoine national. Les gens des villes sont « des traîtres, des vendus » qui semblent faire bon ménage avec l'occupant et s'efforcent dans le cadre du système colonial de réussir. C'est pourquoi on entend souvent dire par les paysans que les gens des villes sont sans moralité. Nous ne nous trouvons pas ici en présence de la classique opposition de la campagne et de la ville. C'est l'opposition entre le colonisé exclu des avantages du colonialisme et celui qui s'arrange pour tirer parti de l'exploitation coloniale.

Les colonialistes utilisent d'ailleurs cette opposition dans leur lutte contre les partis nationalistes. Ils mobilisent les montagnards, les blédards contre les gens de la ville. Ils dressent l'arrière-pays contre les côtes, ils réactivent les tribus et il ne faut pas s'étonner de voir Kalondji se faire couronner roi du Kasaii, comme il ne fallait pas s'étonner il y a quelques années de voir l'Assemblée des chefs du Ghana tenir la dragée haute à N'Krumah. 
Les partis politiques n'arrivent pas à implanter leur organisation dans les campagnes. Au lieu d'utiliser les structures existantes [112] pour leur donner un contenu nationaliste ou progressiste ils entendent, dans le cadre du système colonial, bouleverser la réalité traditionnelle. Ils s'imaginent pouvoir faire démarrer la nation alors que les mailles du système colonial sont encore pesantes. Ils ne vont pas à la rencontre des masses. Ils ne mettent pas leurs connaissances théoriques au service du peuple mais ils tentent d'encadrer les masses selon un schéma a priori. Aussi, de la capitale, vont-ils parachuter dans les villages des dirigeants inconnus ou trop jeunes qui, investis par l'autorité centrale, entendent mener le douar ou le village comme une cellule d'entreprise. Les chefs traditionnels sont ignorés, quelquefois brimés. L'histoire de la nation future piétine avec une singulière désinvolture les petites histoires locales, c'est-à-dire la seule actualité nationale alors qu'il faudrait insérer harmonieusement l'histoire du village, l'histoire des conflits traditionnels des clans et des tribus dans l'action décisive à laquelle on appelle le peuple. Les vieux, entourés de respect dans les sociétés traditionnelles et généralement revêtus d'une autorité morale indiscutable, sont publiquement ridiculisés. Les services de l'occupant ne se font pas faute d'utiliser ces rancunes et se tiennent au courant des moindres décisions adoptées par cette caricature d'autorité. La répression policière, éclairée parce que basée sur des renseignements précis, s'abat. Les dirigeants parachutés et les membres importants de la nouvelle assemblée sont arrêtés.

Les échecs subis confirment «l'analyse théorique » des partis nationalistes. L'expérience désastreuse de tentative d'embrigadement des masses rurales renforce leur méfiance et cristallise leur agressivité contre cette partie du peuple. Après le triomphe de la lutte de libération nationale, les mêmes erreurs se renouvellent, alimentant les tendances décentralisatrices et autonomistes. Le tribalisme de la phase coloniale fait place au régionalisme de la phase nationale, avec son expression institutionnelle: le fédéralisme. 
Mais il se trouve que les masses rurales, malgré le peu d'emprise que les partis nationalistes ont sur elles, interviennent de [113] manière décisive soit dans le processus de maturation de la conscience nationale, soit pour relayer l'action des partis nationalistes, soit plus rarement pour se substituer purement et simplement à la stérilité de ces partis.

La propagande des partis nationalistes trouve toujours un écho au sein des masses paysannes. Le souvenir de la période anticoloniale demeure vivace dans les villages. Les femmes murmurent encore à l'oreille des enfants les chants qui ont accompagné les guerriers résistant à la conquête. À 12, 13 ans les petits villageois connaissent le nom des vieillards qui ont assisté à la dernière insurrection et les rêves dans les douars, dans les villages ne sont pas les rêves de luxe ou de succès aux examens que font les enfants des villes, mais des rêves d'identification à tel ou tel combattant dont le récit de la mort héroïque provoque encore aujourd'hui d'abondantes larmes.

Au moment où les partis nationalistes tentent d'organiser la classe ouvrière embryonnaire des villes, on assiste dans les campagnes à des explosions en apparence absolument inexplicables. C'est par exemple la fameuse insurrection de 1947 à Madagascar. Les services colonialistes sont formels: c'est une jacquerie. En fait nous savons aujourd'hui que les choses, comme toujours, furent beaucoup plus compliquées. Au cours de la Seconde Guerre mondiale les grandes compagnies coloniales étendirent leur puissance et s'emparèrent de la totalité des terres encore libres. Toujours à cette même époque on parla de l'implantation éventuelle dans liîle de réfugiés juifs, kabyles, antillais. La rumeur courut également de l'invasion prochaine de lîle avec la complicité des colons par les Blancs d'Afrique du Sud. Aussi, après la guerre, les candidats de la liste nationaliste furent-ils triomphalement élus. Immédiatement après, la répression s'organisa contre les cellules du parti MDRM (Mouvement démocratique de la rénovation malgache). Le colonialisme, pour parvenir à ses fins, utilisa les moyens les plus classiques: arrestations multiples, propagande raciste intertribale et créa- 
tion d'un parti avec les éléments inorganisés du lumpen-prolétariat. [114] Ce parti dit des Déshérités de Madagascar (PADESM) apportera à l'autorité coloniale, par ses provocations décisives, la caution légale du maintien de l'ordre. Or cette opération banale de liquidation d'un parti préparée à l'avance prend ici des proportions gigantesques. Les masses rurales, sur la défensive depuis trois ou quatre ans, se sentent soudain en danger de mort et décident de s'opposer farouchement aux forces colonialistes. Armé de sagaies et plus souvent de pierres et de bâtons, le peuple se jette dans l'insurrection généralisée en vue de la libération nationale. On connaît la suite.

Ces insurrections armées ne représentent qu'un des moyens utilisés par les masses rurales pour intervenir dans la lutte nationale. Quelquefois les paysans prennent le relais de l'agitation urbaine, le parti nationaliste dans les villes étant l'objet de la répression policière. Les nouvelles parviennent aux campagnes amplifiées, démesurément amplifiées: leaders arrêtés, mitraillages multiples, le sang nègre inonde la ville, les petits colons se baignent dans le sang arabe. Alors la haine accumulée, la haine exacerbée éclate. Le poste de police avoisinant est investi, les gendarmes sont déchiquetés, l'instituteur est massacré, le médecin n'a la vie sauve que parce qu'il était absent, etc. Des colonnes de pacification sont dépêchées sur les lieux, l'aviation bombarde. L'étendard de la révolte est alors déployé, les vieilles traditions guerrières resurgissent, les femmes applaudissent, les hommes s'organisent et prennent position dans les montagnes, la guérilla commence. Spontanément les paysans créent l'insécurité généralisée, le colonialisme prend peur, s'installe dans la guerre ou négocie.

Comment réagissent les partis nationalistes à cette irruption décisive des masses paysannes dans la lutte nationale? Nous avons vu que la majorité des partis nationalistes n'ont pas inscrit dans leur propagande la nécessité de l'action armée. Ils ne s'opposent pas à la persistance de l'insurrection, mais ils se contentent de faire confiance à la 
spontanéité des ruraux. En [115] gros, ils se comportent à l'égard de cet élément nouveau comme s'il s'agissait d'une manne tombée du ciel, priant le sort que ça continue. Ils exploitent cette manne mais ne tentent pas d'organiser l'insurrection. Ils n'envoient pas dans les campagnes des cadres pour politiser les masses, pour éclairer les consciences, pour élever le niveau du combat. Ils espèrent qu'emportée par son mouvement l'action de ces masses ne se ralentira pas. Il n'y a pas contamination du mouvement rural par le mouvement urbain. Chacun évolue selon sa dialectique propre.

Les partis nationalistes ne tentent pas d'introduire dans les masses rurales, qui sont à ce moment entièrement disponibles, des mots d'ordre. Ils ne leur proposent pas d'objectif, ils espèrent simplement que ce mouvement se perpétuera indéfiniment et que les bombardements n'en viendront pas à bout. On voit donc que, même à cette occasion, les partis nationalistes n'exploitent pas la possibilité qui leur est offerte d'intégrer les masses rurales, de les politiser, d'élever le niveau de leur lutte. On maintient la position criminelle de méfiance visà-vis des campagnes.

Les cadres politiques se terrent dans les villes, font comprendre au colonialisme qu'ils n'ont pas de rapport avec les insurgés ou partent à l'étranger. Il arrive rarement qu'ils rejoignent le peuple dans les montagnes. Au Kenya, par exemple, pendant l'insurrection Mau-Mau aucun nationaliste connu n'a revendiqué son appartenance à ce mouvement ou tenté de défendre ces hommes.

Il n'y a pas d'explication féconde, pas de confrontation entre les différentes couches de la nation. Aussi, au moment de l'indépendance, survenue après la répression exercée sur les masses rurales et l'entente entre le colonialisme et les partis nationalistes, retrouvonsnous accentuée cette incompréhension. Les ruraux se montreront réticents à l'égard des réformes de structure proposées par le gouvernement ainsi que des innovations sociales, même objectivement progressistes, parce que précisément les responsables actuels du régime n'ont pas expliqué à l'ensemble du peuple, pendant la période coloniale, les 
objectifs [116] du parti, l'orientation nationale, les problèmes internationaux, etc.

À la méfiance que les ruraux et les féodaux entretenaient à l'égard des partis nationalistes pendant la période coloniale fait suite une hostilité égale pendant la période nationale. Les services secrets colonialistes, qui n'ont pas désarmé après l'indépendance, entretiennent le mécontentement et arrivent encore à créer aux jeunes gouvernements de graves difficultés. Somme toute, le gouvernement ne fait que payer sa paresse de la période de libération et son constant mépris des ruraux. La nation pourra avoir une tête raisonnable, progressiste même, mais le corps immense restera débile, rétif, non coopératif.

La tentation sera alors de briser ce corps en centralisant l'administration et en encadrant fermement le peuple. C'est l'une des raisons pour lesquelles on entend souvent dire que, dans les pays sousdéveloppés, il faut une certaine dose de dictature. Les dirigeants se méfient des masses rurales. C'est le cas par exemple de certains gouvernements qui, bien longtemps après l'indépendance nationale, considèrent l'arrière-pays comme une région non pacifiée où le chef de l'État, les ministres, ne s'aventurent qu'à l'occasion des mancuvres de l'armée nationale. Cet arrière-pays est assimilé pratiquement à l'inconnu. Paradoxalement, le gouvernement national dans son comportement à l'égard des masses rurales rappelle par certains traits le pouvoir colonial. "On ne sait pas très bien comment ces masses vont réagir » et les jeunes dirigeants n'hésitent pas à dire: « Il faut la trique, si l'on veut sortir ce pays du Moyen Âge. » Mais, nous l'avons vu, la désinvolture avec laquelle les partis politiques ont agi avec les masses rurales pendant la phase coloniale ne pouvait qu'être préjudiciable à l'unité nationale, au démarrage accéléré de la nation.

Quelquefois le colonialisme tente de diversifier, de disloquer la poussée nationaliste. Au lieu de dresser les cheiks et les chefs contre les « révolutionnaires» des villes, les bureaux indigènes [117] organi- 
sent les tribus et les confréries en partis. Face au parti urbain qui commençait à « incarner la volonté nationale » et à constituer un danger pour le régime colonial, des groupuscules prennent naissance, des tendances, des partis à base ethnique ou régionaliste surgissent. C'est la tribu dans son intégralité qui se mue en parti politique conseillé de près par les colonialistes. La table ronde peut commencer. Le parti unitaire sera noyé dans l'arithmétique des tendances. Les partis tribaux s'opposent à la centralisation, à l'unité et dénoncent la dictature du parti unitaire.

Plus tard, cette tactique sera utilisée par l'opposition nationale. Parmi les deux ou trois partis nationalistes qui ont mené la lutte de libération, l'occupant a fait son choix. Les modalités de ce choix sont classiques : lorsqu'un parti a fait l'unanimité nationale et s'est imposé à l'occupant comme seul interlocuteur, l'occupant multiplie les manœuvres et retarde au maximum l'heure des négociations. Ce retard sera utilisé à émietter les exigences de ce parti ou à obtenir de la direction la mise à l'écart de certains éléments « extrémistes».

Si, par contre, aucun parti ne s'est véritablement imposé, l'occupant se contente de privilégier celui qui lui paraît le plus «raisonnable». Les partis nationalistes qui n'ont pas participé aux négociations se lancent alors dans une dénonciation de l'accord intervenu entre l'autre parti et l'occupant. Le parti qui reçoit le pouvoir de l'occupant, conscient du danger que constituent les positions strictement démagogiques et confuses du parti rival, tente de le démanteler et le condamne à l'illégalité. Le parti persécuté n'a d'autre ressource que de se réfugier à la périphérie des villes et dans les campagnes. Il essaie de soulever les masses rurales contre les «vendus de la côte et les corrompus de la capitale». Tous les prétextes sont alors utilisés : arguments religieux, dispositions novatrices prises par la nouvelle autorité nationale et qui rompent avec la tradition. On exploite la tendance obscurantiste des masses rurales. La doctrine dite révolutionnaire repose en fait sur le caractère rétrograde, passionnel [118] et spontanéiste des campagnes. On murmure çà et là que la montagne bouge, que les cam- 
pagnes sont mécontentes. On affirme que dans tel coin la gendarmerie a ouvert le feu sur des paysans, que des renforts ont été envoyés, que le régime est près de s'écrouler. Les partis d'opposition, sans programme clair, n'ayant d'autre but que de se substituer à l'équipe dirigeante, remettent leur destin entre les mains spontanées et obscures des masses paysannes.

Inversement, il arrive que l'opposition ne s'appuie plus sur les masses rurales mais sur les éléments progressistes, les syndicats de la jeune nation. Dans ce cas, le gouvernement fait appel aux masses pour résister aux revendications des travailleurs, dénoncées alors comme manouvres d'aventuriers antitraditionalistes. Les constatations que nous avons eu l'occasion de faire au niveau des partis politiques se retrouvent, mutatis mutandis, au niveau des syndicats. Au début, les formations syndicales dans les territoires coloniaux sont régulièrement des branches locales des syndicats métropolitains et les mots d'ordre répondent en écho à ceux de la métropole.

La phase décisive de la lutte de libération se précisant, quelques syndicalistes indigènes vont décider la création de syndicats nationaux. L'ancienne formation, importée de la métropole, sera massivement désertée par les autochtones. Cette création syndicale est un nouvel élément de pression pour les populations urbaines sur le colonialisme. Nous avons dit que le prolétariat aux colonies est embryonnaire et représente la fraction du peuple la plus favorisée. Les syndicats nationaux nés dans la lutte s'organisent dans les villes et leur programme est avant tout un, programme politique, un programme nationaliste. Mais ce syndicat national né au cours de la phase décisive du combat pour l'indépendance est en fait l'enrégimentement légal des éléments nationalistes conscients et dynamiques.

Les masses rurales, dédaignées par les partis politiques, continuent à être tenues à l'écart. Il y aura, bien sûr, un syndicat des travailleurs agricoles mais cette création se contente de [119] répondre à la nécessité formelle de «présenter un front uni au colonialisme». Les 
responsables syndicaux qui ont fait leurs armes dans le cadre des formations syndicales métropolitaines ne savent pas organiser les masses urbaines. Ils ont perdu tout contact avec la paysannerie et se préoccupent en premier lieu de l'embrigadement des métallos, des dockers, des fonctionnaires du gaz et de l'électricité, etc.

Pendant la phase coloniale, les formations syndicales nationalistes constituent une force de frappe spectaculaire. Dans les villes, les syndicats peuvent immobiliser, en tout cas enrayer à n'importe quel moment, l'économie colonialiste. Comme le peuplement européen est souvent cantonné dans les villes, les répercussions psychologiques des manifestations sur ce peuplement sont considérables: pas d'électricité, pas de gaz, les ordures ne sont pas ramassées, les marchandises pourrissent sur les quais.

Ces îlots métropolitains que constituent les villes dans le cadre colonial ressentent profondément l'action syndicale. La forteresse du colonialisme représentée par la capitale, supporte difficilement ces coups de boutoir. Mais «l'intérieur» (les masses rurales) demeure étranger à cette confrontation.

Ainsi, on le voit, il y a une disproportion du point de vue national entre l'importance des syndicats et le reste de la nation. Après l'indépendance, les ouvriers embrigadés dans les syndicats ont l'impression de tourner à vide. L'objectif limité qu'ils s'étaient fixé se révèle, au moment même où il est atteint, bien précaire eu égard à l'immensité de la tâche de construction nationale. Face à la bourgeoisie nationale dont les relations avec le pouvoir sont souvent très étroites, les dirigeants syndicaux découvrent qu'ils ne peuvent plus se cantonner dans l'agitation ouvriériste. Congénitalement isolés des masses rurales, incapables de diffuser des mots d'ordre au-delà des faubourgs, les syndicats adoptent des positions de plus en plus politiques. En fait, les syndicats sont candidats au pouvoir. Ils tentent par tous les moyens d'acculer la bourgeoisie: protestation contre le maintien des bases étrangères sur le territoire national, dénonciation [120] des accords commerciaux, prises de position contre la politique extérieure du gouvernement national. Les ouvriers maintenant « indépendants» tour- 
nent à vide. Les syndicats s'aperçoivent au lendemain de l'indépendance que les revendications sociales si elles étaient exprimées scandaliseraient le reste de la nation. Les ouvriers sont en effet les favorisés du régime. Ils représentent la fraction la plus aisée du peuple. Une agitation qui se proposerait d'arracher l'amélioration des conditions de vie pour les ouvriers et les dockers serait non seulement impopulaire, mais risquerait encore de provoquer l'hostilité des masses déshéritées des campagnes. Les syndicats, à qui tout syndicalisme est interdit, piétinent sur place.

Ce malaise traduit la nécessité objective d'un programme social intéressant enfin l'ensemble de la nation. Les syndicats découvrent subitement que l'arrière-pays doit être également éclairé et organisé. Mais, parce qu'à aucun moment ils ne se sont préoccupés de mettre en place des courroies de transmission entre eux et les masses paysannes, et comme précisément ces masses constituent les seules forces spontanément révolutionnaires du pays, les syndicats vont faire la preuve de leur inefficacité et découvrir le caractère anachronique de leur programme.

Les dirigeants syndicaux, plongés dans l'agitation politicoouvriériste, en arrivent mécaniquement à la préparation d'un coup d'État. Mais, là encore, l'intérieur est exclu. C'est une explication limitée entre la bourgeoisie nationale et l'ouvriérisme syndical. La bourgeoisie nationale, reprenant les vieilles traditions du colonialisme, montre ses forces militaires et policières, tandis que les syndicats organisent des meetings, mobilisent des dizaines de milliers d'adhérents. Les paysans, face à cette bourgeoisie nationale et à ces ouvriers qui, somme toute, mangent à leur faim, regardent en haussant les épaules. Les paysans haussent les épaules, car ils se rendent compte que les uns et les autres les considèrent comme une force d'appoint. Les syndicats, les partis ou le gouvernement, dans une sorte de machiavélisme immoral, utilisent les masses paysannes comme force de manœuvre inerte, aveugle. Comme force brute. 
Dans certaines circonstances par contre, les masses paysannes vont intervenir de façon décisive, à la fois dans la lutte de libération nationale et dans les perspectives que se choisit la nation future. Ce phénomène revêt pour les pays sous-développés une importance fondamentale: c'est pourquoi nous nous proposons de l'étudier en détail.

Nous avons vu que, dans les partis nationalistes, la volonté de briser le colonialisme fait bon ménage avec une autre volonté: celle de s'entendre à l'amiable avec lui. Au sein de ces partis, deux processus vont quelquefois se produire. D'abord, des éléments intellectuels ayant procédé à une analyse soutenue de la réalité coloniale et de la situation internationale commenceront à critiquer le vide idéologique du parti national et son indigence tactique et stratégique. Ils se mettent à poser inlassablement aux dirigeants, des questions cruciales: «Qu'estce que le nationalisme? Que mettez-vous derrière ce mot? Que contient ce vocable? L'indépendance pour quoi? Et d'abord comment pensez-vous y parvenir ? » tout en exigeant que les problèmes méthodologiques soient abordés avec vigueur. Aux moyens électoralistes, ils vont suggérer d'adjoindre « tout autre moyen ». Aux premières escarmouches, les dirigeants se débarrassent vite de ce bouillonnement qu'ils qualifient volontiers de juvénile. Mais, parce que ces revendications ne sont ni l'expression d'un bouillonnement, ni la marque de la jeunesse les éléments révolutionnaires qui défendent ces positions vont être rapidement isolés. Les dirigeants drapés dans leur expérience vont rejeter impitoyablement « ces aventuriers, ces anarchistes».

La machine du parti se montre rebelle à toute innovation. La minorité révolutionnaire se retrouve seule, face à une direction apeurée et angoissée à l'idée qu'elle pourrait être emportée dans une tourmente dont elle n'imagine même pas les aspects, la force ou l'orientation. Le deuxième processus a trait aux cadres dirigeants ou subalternes qui, du fait de leurs activités, ont été en butte aux persécutions policières colonialistes. Ce qu'il est intéressant de signaler, c'est que ces hommes sont arrivés aux [122] sphères dirigeantes du parti par leur travail obstiné, l'esprit de sacrifice et un patriotisme exemplaire. Ces hommes, venus de la base, sont souvent de petits mancuvres, des travail- 
leurs saisonniers et même quelquefois d'authentiques chômeurs. Pour eux, militer dans un parti national, ce n'est pas faire de la politique, c'est choisir le seul moyen de passer de l'état animal à l'état humain. Ces hommes, que gêne le légalisme exacerbé du parti, vont révéler dans les limites des activités qui leur sont confiées un esprit d'initiative, un courage et un sens de la lutte qui presque mécaniquement les désignent aux forces de répression du colonialisme. Arrêtés, condamnés, torturés, amnistiés, ils utilisent la période de détention à confronter leurs idées et à durcir leur détermination. Dans les grèves de la faim, dans la solidarité violente des fosses communes des prisons, ils vivent leur libération comme une occasion qui leur sera donnée de déclencher la lutte armée. Mais dans le même temps, au dehors, le colonialisme qui commence à être assailli de partout fait des avances aux modérés nationalistes.

On assiste donc à un écartèlement proche de la rupture entre la tendance illégaliste et la tendance légaliste du parti. Les illégaux se sentent indésirables. On les fuit. En prenant d'infinies précautions, les légaux du parti leur viennent en aide mais déjà ils se sentent étrangers. Ces hommes vont alors entrer en contact avec les éléments intellectuels dont ils avaient pu apprécier les positions quelques années auparavant. Un parti clandestin, latéral au parti légal, consacre cette rencontre. Mais la répression contre ces éléments irrécupérables s'intensifie à mesure que le parti légal se rapproche du colonialisme en tentant de le modifier « de l'intérieur». L'équipe illégale se trouve alors dans un cul-de-sac historique.

Rejetés des villes, ces hommes se groupent, dans un premier temps, dans les banlieues périphériques. Mais le filet policier les y déniche et les contraint à quitter définitivement les villes, à fuir les lieux de la lutte politique. Ils se rejettent vers les campagnes, vers les montagnes, vers les masses paysannes. Dans un [123] premier temps, les masses se referment sur eux en les soustrayant à la recherche policière. Le militant nationaliste qui décide, au lieu de jouer à cachecache avec les policiers dans les cités urbaines, de remettre son des- 
tin entre les mains des masses paysannes ne perd jamais. Le manteau paysan se referme sur lui avec une tendresse et une vigueur insoupçonnées. Véritables exilés de l'intérieur, coupés du milieu urbain au sein duquel ils avaient précisé les notions de nation et de lutte politique, ces hommes sont devenus en fait des maquisards. Obligés tout le temps de se déplacer pour échapper aux policiers, marchant la nuit pour ne pas attirer l'attention, ils vont avoir l'occasion de parcourir, de connaître leur pays. Oubliés les cafés, les discussions sur les prochaines élections, la méchanceté de tel policier. Leurs oreilles entendent la vraie voix du pays et leurs yeux voient la grande, l'infinie misère du peuple. Ils se rendent compte du temps précieux qui a été perdu en vains commentaires sur le régime colonial. Ils comprennent enfin que le changement ne sera pas une réforme, ne sera pas une amélioration. Ils comprennent, dans une sorte de vertige qui ne cessera plus de les habiter, que l'agitation politique dans les villes sera toujours impuissante à modifier, à bouleverser le régime colonial.

Ces hommes prennent l'habitude de parler aux paysans. Ils découvrent que les masses rurales n'ont jamais cessé de poser le problème de leur libération en termes de violence, de terre à reprendre aux étrangers, de lutte nationale, d'insurrection armée. Tout est simple. Ces hommes découvrent un peuple cohérent qui se perpétue dans une sorte d'immobilité mais qui garde intacts ses valeurs morales, son attachement à la nation. Ils découvrent un peuple généreux, prêt au sacrifice, désireux de se donner, impatient et d'une fierté de pierre. On comprend que la rencontre de ces militants traqués par la police et de ces masses piaffantes, et d'instinct rebelles puisse donner un mélange détonant d'une puissance inaccoutumée. Les hommes venus des villes se mettent à l'école du peuple et dans le même temps ouvrent, à l'intention du peuple, des cours de formation politique et militaire. Le peuple fourbit ses armes. En fait, les [124] cours ne durent pas longtemps car les masses, reprenant contact avec l'intimité même de leurs muscles, amènent les dirigeants à brusquer les choses. La lutte armée est déclenchée. 
L'insurrection désoriente les partis politiques. Leur doctrine, en effet, a toujours affirmé l'inefficacité de toute épreuve de force et leur existence même est une constante condamnation de toute insurrection. Secrètement, certains partis politiques partagent l'optimisme des colons et se félicitent d'être en dehors de cette folie dont on dit qu'elle sera réprimée dans le sang. Mais le feu allumé, comme une épidémie galopante, se propage à l'ensemble du pays. Les blindés et les avions ne remportent pas les succès escomptés. Devant l'étendue du mal, le colonialisme commence à réfléchir. Au sein même du peuple qui opprime, des voix se font entendre qui attirent l'attention sur la gravité de la situation.

Le peuple quant à lui, dans ses huttes et dans ses rêves, se met en communication avec le nouveau rythme national. $\grave{A}$ voix basse, au milieu de son cœur, il chante aux glorieux combattants des hymnes interminables. L'insurrection a déjà envahi la nation. C'est au tour des partis d'être maintenant isolés.

Pourtant les dirigeants de l'insurrection prennent conscience, un jour ou l'autre, de la nécessité d'étendre cette insurrection aux villes. Cette prise de conscience n'est pas fortuite. Elle consacre la dialectique qui préside au développement d'une lutte armée de libération nationale. Quoique les campagnes représentent des réserves inépuisables d'énergie populaire et que les groupes armés y fassent régner l'insécurité, le colonialisme ne doute pas réellement de la solidité de son système. Il ne se sent pas fondamentalement en danger. Le dirigeant de l'insurrection décide donc de porter la guerre chez l'ennemi, c'est-à-dire dans les cités tranquilles et grandiloquentes.

L'installation de l'insurrection dans les cités pose à la direction des problèmes difficiles. On a vu que la plupart des dirigeants, nés ou ayant grandi dans les villes, avaient fui leur [125] milieu naturel, parce que pourchassés par la police colonialiste et généralement incompris des cadres prudents et raisonnables des partis politiques. Leur retraite dans les campagnes a été à la fois une fuite devant la répression et une méfiance à l'égard des vieilles formations politiques. Les antennes urbaines naturelles de ces dirigeants sont les nationalistes connus au 
sein des partis politiques. Mais, précisément, nous avons vu que leur histoire récente s'était déroulée latéralement à ces dirigeants timorés et crispés dans une réflexion ininterrompue sur les méfaits du colonialisme.

D'ailleurs, les premières tentatives que les hommes du maquis feront en direction de leurs anciens amis, ceux précisément qu'ils estiment être le plus à gauche, confirmeront leurs appréhensions et leur enlèveront jusqu'au désir même de revoir les vieilles connaissances. En fait l'insurrection, partie des campagnes, va pénétrer dans les villes par la fraction de la paysannerie bloquée à la périphérie urbaine, celle qui n'a pu encore trouver un os à ronger dans le système colonial. Les hommes que la population croissante des campagnes, l'expropriation coloniale ont amenés à déserter la terre familiale tournent inlassablement autour des différentes villes, espérant qu'un jour ou l'autre on leur permettra d'y entrer. C'est dans cette masse, c'est dans ce peuple des bidonvilles, au sein du lumpen-prolétariat que l'insurrection va trouver son fer de lance urbain. Le lumpen-prolétariat constitue l'une des forces le plus spontanément et le plus radicalement révolutionnaires d'un peuple colonisé.

Au Kenya, dans les années qui ont précédé la révolte des Mau-Mau, on a vu les autorités coloniales britanniques multiplier les mesures d'intimidation contre le lumpen-prolétariat. Forces de police et missionnaires ont coordonné leurs efforts, dans les années 1950-1951, pour répondre comme il convenait à l'afflux énorme de jeunes Kenyans venus des campagnes et des forêts et qui, n'arrivant pas à se placer sur le marché, volaient, se livraient à la débauche, à l'alcoolisme, etc. La délinquance juvénile dans les pays colonisés est le produit direct de l'existence [126] du lumpen-prolétariat. Pareillement, au Congo, des mesures draconiennes furent prises à partir de 1957 pour refouler dans les campagnes les « jeunes voyous » qui perturbaient l'ordre établi. Des camps de recasement furent ouverts et confiés aux missions évangéliques sous la protection, bien sûr, de l'armée belge. 
La constitution d'un lumpen-prolétariat est un phénomène qui obéit à une logique propre et ni l'activité débordante des missionnaires, ni les arrêtés du pouvoir central ne peuvent enrayer sa progression. Ce lumpen-prolétariat, pareil à une meute de rats, malgré les coups de pied, malgré les coups de pierres, continue à ronger les racines de l'arbre.

Le bidonville consacre la décision biologique du colonisé d'envahir coûte que coûte, et s'il le faut par les voies les plus souterraines, la citadelle ennemie. Le lumpen-prolétariat constitué et pesant de toutes ses forces sur la « sécurité 》 de la ville signifie le pourrissement irréversible, la gangrène installée au cœur de la domination coloniale. Alors les souteneurs, les voyous, les chômeurs, les droit commun, sollicités, se jettent dans la lutte de libération comme de robustes travailleurs. Ces désceuvrés, ces déclassés vont, par le canal de l'action militante et décisive retrouver le chemin de la nation. Ils ne se réhabilitent pas vis-à-vis de la société coloniale ou de la morale du dominateur. Tout au contraire, ils assument leur incapacité à entrer dans la cité autrement que par la force de la grenade ou du revolver. Ces chômeurs et ces sous-hommes se réhabilitent vis-à-vis d'eux-mêmes et vis-à-vis de I'histoire. Les prostituées elles aussi, les bonnes à 2000 francs, les désespérées, tous ceux et toutes celles qui évoluent entre la folie et le suicide vont se rééquilibrer, vont se remettre en marche et participer de façon décisive à la grande procession de la nation réveillée.

Les partis nationalistes ne comprennent pas ce phénomène nouveau qui précipite leur désagrégation. L'irruption de l'insurrection dans les villes modifie la physionomie de la lutte. Alors que les troupes colonialistes étaient tout entières tournées vers les campagnes, les voici qui refluent précipitamment vers les [127] villes pour assurer la sécurité des personnes et des biens. La répression disperse ses forces, le danger est partout présent. C'est le sol national, c'est l'ensemble de la colonie qui entrent en transes. Les groupes armés paysans assistent au desserrement de l'étreinte militaire. L'insurrection dans les villes est un ballon d'oxygène inespéré. 
Les dirigeants de l'insurrection, qui voient le peuple enthousiaste et ardent porter des coups décisifs à la machine colonialiste, renforcent leur méfiance à l'égard de la politique traditionnelle. Chaque succès remporté légitime leur hostilité à l'égard de ce que désormais ils appellent le gargarisme, le verbalisme, la «blagologie», l'agitation stérile. Ils ressentent une haine de la «politique», de la démagogie. C'est pourquoi au début nous assistons à un véritable triomphe du culte de la spontanéité.

Les multiples jacqueries nées dans les campagnes attestent, partout où elles éclatent, de la présence ubiquitaire et généralement dense de la nation. Chaque colonisé en armes est un morceau de la nation désormais vivante. Ces jacqueries mettent en danger le régime colonial, mobilisent ses forces en les dispersant, menaçant à tout instant de les asphyxier. Elles obéissent à une doctrine simple: faites que la nation existe. Il n'y a pas de programme, il n'y a pas de discours, il n'y a pas de résolutions, il n'y a pas de tendances. Le problème est clair : il faut que les étrangers partent. Constituons un front commun contre l'oppresseur et renforçons ce front par la lutte armée.

Tant que dure l'inquiétude du colonialisme, la cause nationale progresse et devient la cause de chacun. L'entreprise de libération se dessine et concerne déjà l'ensemble du pays. Dans cette période, le spontané est roi. L'initiative est localisée. Sur chaque piton, un gouvernement en miniature se constitue et assume le pouvoir. Dans les vallées et dans les forêts, dans la jungle et dans les villages, partout, on rencontre une autorité nationale. Chacun par son action fait exister la nation et s'engage à la faire localement triompher. Nous avons affaire à une stratégie de l'immédiateté totalitaire et radicale. Le but, le programme [128] de chaque groupe spontanément constitué est la libération locale. Si la nation est partout, alors elle est ici. Un pas de plus et elle n'est qu'ici. La tactique et la stratégie se confondent. L'art politique se transforme tout simplement en art militaire. Le militant politique, c'est le combattant. Faire la guerre et faire de la politique, c'est une seule et même chose. 
Ce peuple déshérité, habitué à vivre dans le cercle étroite des luttes et des rivalités, va procéder dans une atmosphère solennelle à la toilette et à la purification du visage local de la nation. Dans une véritable extase collective, des familles ennemies décident de tout effacer, de tout oublier. Les réconciliations se multiplient. Les haines tenaces et enfouies sont réveillées pour être plus sûrement extirpées. L'assomption de la nation fait avancer la conscience. L'unité nationale est d'abord l'unité du groupe, la disparition des vieilles querelles et la liquidation définitive des réticences. Dans le même temps, la purification englobera les quelques autochtones qui, par leurs activités, par leur complicité avec l'occupant ont déshonoré le pays. Par contre, les traîtres et les vendus seront jugés et châtiés. Le peuple dans cette marche continue qu'il a entreprise légifère, se découvre et se veut souverain. Chaque point ainsi réveillé du sommeil colonial vit à une température insupportable. Une effusion permanente règne dans les villages, une générosité spectaculaire, une bonté désarmante, une volonté jamais démentie de mourir pour la « cause ». Tout cela évoque à la fois une confrérie, une église, une mystique. Aucun autochtone ne peut rester indifférent à ce nouveau rythme qui entraîne la nation. Des émissaires sont dépêchés aux tribus avoisinantes. Ils constituent le premier système de liaison de l'insurrection et apportent cadence et mouvement aux régions encore immobiles. Des tribus dont la rivalité opiniâtre est pourtant bien connue désarment, dans l'allégresse et dans les larmes, et se jurent assistance et soutien. Dans le coude à coude fraternel, dans la lutte armée, les hommes rejoignent leurs ennemis d'hier. Le cercle national s'agrandit et ce sont de nouvelles embuscades qui saluent l'entrée [129] en scène de nouvelles tribus. Chaque village se découvre agent absolu et relais. La solidarité intertribale, inter-villages, la solidarité nationale se déchiffrent d'abord dans la multiplication des coups portés à l'ennemi. Chaque nouveau groupe qui se constitue, chaque salve nouvelle qui éclate indiquent que chacun traque l'ennemi, que chacun fait face. 
Cette solidarité va se manifester beaucoup plus clairement au cours de la seconde période qui se caractérise par le déclenchement de l'offensive ennemie. Les forces coloniales, après l'explosion, se regroupent, se réorganisent et inaugurent des méthodes de combat correspondant à la nature de l'insurrection. Cette offensive va remettre en question l'atmosphère euphorique et paradisiaque de la première période. L'ennemi déclenche l'attaque et concentre sur des points précis des forces importantes. Le groupe local est très rapidement débordé. Il l'est d'autant plus qu'il a tendance au début à accepter de front le combat. L'optimisme qui a régné dans la première période rend le groupe intrépide, voire inconscient. Le groupe qui s'est persuadé que son piton est la nation n'accepte pas de décrocher, ne supporte pas de battre en retraite. Les pertes sont nombreuses et le doute s'infiltre massivement dans les esprits. Le groupe subit l'assaut local comme une épreuve décisive. Il se comporte littéralement comme si le sort du pays se jouait ici et maintenant.

Mais, on l'a compris, cette impétuosité volontariste qui entend régler son sort tout de suite au système colonial est condamnée, en tant que doctrine de l'instantanéisme, à se nier. Le réalisme le plus quotidien, le plus pratique fait place aux effusions d'hier et se substitue à l'illusion d'éternité. La leçon des faits, les corps fauchés par la mitraille provoquent une réinterprétation globale des événements. Le simple instinct de survie commande une attitude plus mouvante, plus mobile. Cette modification dans la technique de combat est caractéristique des premiers mois de la guerre de libération du peuple angolais. On se souvient que, le 15 mars 1961, les paysans angolais se sont [130] lancés par groupe de deux ou trois mille contre les positions portugaises. Hommes, femmes et enfants, armés ou non armés, avec leur courage, leur enthousiasme, se sont rués en masses compactes et par vagues successives sur des régions où dominaient le colon, le soldat et le drapeau portugais. Des villages, des aérodromes ont été encerclés et ont subi des assauts multiples, mais aussi des milliers d'Angolais ont été fauchés par la mitraille colonialiste. Il n'a pas fallu longtemps aux chefs de l'insurrection angolaise pour comprendre qu'ils devaient trou- 
ver autre chose s'ils voulaient réellement libérer leur pays. Aussi, depuis quelques mois, le leader angolais Holden Roberto a-t-il réorganisé l'Armée nationale angolaise en tenant compte des différentes guerres de libération et en utilisant les techniques de guérilla.

Dans la guérilla en effet, la lutte n'est plus où l'on est mais où l'on va. Chaque combattant emporte la patrie en guerre entre ses orteils nus. L'armée de libération nationale n'est pas celle qui est aux prises une fois pour toutes avec l'ennemi mais celle qui va de village en village, qui se replie dans les forêts et qui trépigne de joie quand est aperçu dans la vallée le nuage de poussière soulevé par les colonnes adverses. Les tribus se mettent en branle, les groupes se déplacent, changeant de terrain. Les gens du nord font mouvement vers l'ouest, ceux de la plaine se hissent dans les montagnes. Aucune position stratégique n'est privilégiée. L'ennemi s'imagine nous poursuivre mais nous nous arrangeons toujours pour être sur ses arrières, le frappant au moment même où il nous croit anéantis. Désormais, c'est nous qui le poursuivons. Avec toute sa technique et sa puissance de feu, l'ennemi donne l'impression de patauger et de s'enliser. Nous chantons, nous chantons.

Entre-temps cependant, les dirigeants de l'insurrection comprennent qu'il faut éclairer les groupes, les instruire, les endoctriner, créer une armée, centraliser l'autorité. L'émiettement de la nation, qui manifestait la nation en armes, demande à être corrigé et dépassé. Les dirigeants qui avaient fui l'atmosphère de vaine politique des villes redécouvrent la politique, non plus [131] comme technique d'endormissement ou de mystification mais comme moyen unique d'intensifier la lutte et de préparer le peuple à la direction lucide du pays. Les dirigeants de l'insurrection s'aperçoivent que les jacqueries, même grandioses, demandent à être contrôlées et orientées. Les dirigeants sont amenés à nier le mouvement en tant que jacquerie, le transformant ainsi en guerre révolutionnaire. Ils découvrent que le succès de la lutte suppose la clarté des objectifs, la netteté de la méthodologie et surtout la connaissance par les masses de la dynamique temporelle de leurs efforts. On tient trois jours, à la rigueur trois mois, en utilisant la dose de ressentiment contenue dans les masses 
mais on ne triomphe pas dans une guerre nationale, on ne met pas en déroute la terrible machine de l'ennemi, on ne transforme pas les hommes si l'on oublie d'élever la conscience du combattant. Ni l'acharnement dans le courage, ni la beauté des slogans ne suffisent.

Le développement de la guerre de libération se charge d'ailleurs de porter un coup décisif à la foi des dirigeants. L'ennemi, en effet, modifie sa tactique. À la politique brutale de répression il allie opportunément les gestes spectaculaires de détente, les manœuvres de division, "l'action psychologique ». Il tente çà et là et avec succès de redonner vie aux luttes tribales, utilisant les provocateurs, faisant ce que l'on appelle de la contre-subversion. Le colonialisme emploiera pour réaliser ses objectifs deux catégories d'autochtones. Et d'abord, les traditionnels collaborateurs, chefs, caïds, sorciers. Les masses paysannes plongées, nous l'avons vu, dans la répétition sans histoire d'une existence immobile continuent à vénérer les chefs religieux, les descendants des vieilles familles. La tribu, comme un seul homme, s'engage dans la voie qui lui est désignée par le chef traditionnel. À coups de prébendes, à prix d'or, le colonialisme s'attachera les services de ces hommes de confiance.

Le colonialisme va trouver également dans le lumpen-prolétariat une masse de mancuure considérable. Aussi tout mouvement de libération nationale doit-il apporter le maximum [132] d'attention à ce lumpenprolétariat. Celui-ci répond toujours à l'appel de l'insurrection, mais si l'insurrection croit pouvoir se développer en l'ignorant, le lumpenprolétariat, cette masse d'affamés et de déclassés, se jettera dans la lutte année, participera au conflit aux côtés, cette fois, de l'oppresseur. L'oppresseur, qui ne perd jamais une occasion de faire se bouffer les nègres entre eux, utilisera avec un rare bonheur l'inconscience et l'ignorance qui sont les tares du lumpen-prolétariat. Cette réserve humaine disponible, si elle n'est pas immédiatement organisée par l'insurrection, se retrouvera comme mercenaires aux cotés des troupes colonialistes. En Algérie, c'est le lumpen-prolétariat qui a fourni les harkis et les messalistes; en Angola, c'est lui qui a donné ces 
ouvreurs de routes qui précèdent aujourd'hui les colonnes armées portugaises: au Congo, on retrouve le lumpen-prolétariat dans les manifestations régionalistes du Kasaï et du Katanga, tandis qu'à Léopoldville il fut utilisé par les ennemis du Congo pour organiser des meetings « spontanés » anti-lumumbistes.

L'adversaire, qui analyse les forces de l'insurrection, qui étudie de mieux en mieux l'ennemi global que constitue le peuple colonisé, se rend compte de la faiblesse idéologique, de l'instabilité spirituelle de certaines couches de la population. L'adversaire découvre, latéralement à une avant-garde insurrectionnelle rigoureuse et bien structurée, une masse d'hommes dont l'engagement risque constamment d'être remis en question par une trop grande habitude de la misère physiologique, des humiliations et de l'irresponsabilité. L'adversaire utilisera cette masse, quitte à payer le prix fort. Il créera du spontané à coups de baïonnettes ou de châtiments exemplaires. Les dollars et les francs belges se déversent sur le Congo tandis qu'à Madagascar se multiplient les exactions anti-Hova et qu'en Algérie des recrues, authentiques otages, sont enrôlées dans les forces françaises. À le lettre, le dirigeant de l'insurrection voit chavirer la nation. Des tribus entières se constituent en harkis et, dotées d'armes modernes, prennent le sentier de la guerre et envahissent la tribu rivale, étiquetée pour la circonstance nationaliste. [133] L'unanimité dans le combat, si féconde et si grandiose aux premières heures de l'insurrection, s'altère. L'unité nationale s'effrite, l'insurrection est à un tournant décisif. La politisation des masses est alors reconnue comme nécessité historique.

Ce volontarisme spectaculaire qui entendait mener d'un seul coup le peuple colonisé à la souveraineté absolue, cette certitude que l'on avait de charrier avec soi à la même allure et sous le même éclairage tous les morceaux de la nation, cette force qui fondait l'espoir se révèlent être à l'expérience une très grande faiblesse. Tant qu'il s'imaginait pouvoir passer sans transition de l'état de colonisé à l'état de citoyen souverain d'une nation indépendante, tant qu'il se prenait au mirage de l'immédiateté de ses muscles, le colonisé ne réalisait pas de véritables 
progrès dans la voie de la connaissance. Sa conscience restait rudimentaire. Le colonisé s'engage dans la lutte avec passion, nous l'avons vu, surtout si cette lutte est armée. Les paysans se sont lancés dans l'insurrection avec d'autant plus d'enthousiasme qu'ils n'avaient point cessé de se crisper sur un mode de vie pratiquement anticolonial. De toute éternité et à la suite de ruses multiples, de rééquilibrations évoquant les prouesses du prestidigitateur, les paysans avaient relativement préservé leur subjectivité de l'imposition coloniale. Ils en arrivaient à croire que le colonialisme n'était pas vraiment vainqueur. L'orgueil du paysan, sa réticence à descendre dans les villes, à côtoyer le monde édifié par l'étranger, ses perpétuels mouvements de recul à l'approche des représentants de l'administration coloniale ne cessaient de signifier qu'il opposait à la dichotomie du colon sa propre dichotomie.

Le racisme antiraciste, la volonté de défendre sa peau qui caractérise la réponse du colonisé à l'oppression coloniale représentent évidemment des raisons suffisantes pour s'engager dans la lutte. Mais on ne soutient pas une guerre, on ne subit pas une répression énorme, on n'assiste pas à la disparition de toute sa famille pour faire triompher la haine ou le racisme. Le racisme, la haine, le ressentiment, " le désir légitime de vengeance » [134] ne peuvent alimenter une guerre de libération. Ces éclairs dans la conscience qui jettent le corps dans des chemins tumultueux, qui le lancent dans un onirisme quasi pathologique où la face de l'autre m'invite au vertige, où mon sang appelle le sang de l'autre, où ma mort par simple inertie appelle la mort de l'autre, cette grande passion des premières heures se disloque si elle entend se nourrir de sa propre substance. Il est vrai que les interminables exactions des forces colonialistes réintroduisent les éléments émotionnels dans la lutte, donnent au militant de nouveaux motifs de haine, de nouvelles raisons de partir à la recherche du «colon à abattre». Mais le dirigeant se rend compte jour après jour que la haine ne saurait constituer un programme. On ne peut, sinon par perversion, faire confiance à l'adversaire qui évidemment se débrouille toujours pour multiplier les crimes, approfondir le «fossé », rejetant ainsi le peuple global du côté 
de l'insurrection. En tout état de cause, l'adversaire, nous l'avons signalé, tâche de gagner la sympathie de certains groupes de la population, de certaines régions, de certains chefs. Au cours de la lutte, des consignes sont données aux colons et aux forces de police. Le comportement se nuance, « s'humanise ». On ira même jusqu'à introduire dans les rapports colon-colonisé des locutions telles que Monsieur ou Madame. On multipliera les politesses, les prévenances. Concrètement, le colonisé a l'impression d'assister à un changement.

Le colonisé qui n'a pas pris seulement les armes parce qu'il mourait de faim et qu'il assistait à la désagrégation de sa société, mais aussi parce que le colon le considérait comme une bête, le traitait comme une bête, se montre très sensible à ces mesures. La haine est désamorcée par ces trouvailles psychologiques. Les technologues et les sociologues éclairent les manœuvres colonialistes et multiplient les études sur les «complexes»: complexe de frustration, complexe belliqueux, complexe de colonisabilité. On promeut l'indigène, on essaie de le désarmer par la psychologie et, naturellement, quelques pièces de monnaie. [135] Ces mesures misérables, ces réparations de façade, d'ailleurs savamment dosées, arrivent à remporter certains succès. La faim du colonisé est telle, sa faim de n'importe quoi qui l'humanise même au rabais - est à ce point incoercible que ces aumônes parviennent localement à l'ébranler. Sa conscience est d'une telle précarité, d'une telle opacité, qu'elle s'émeut à la moindre étincelle. La grande soif de lumière indifférenciée du début est menacée à tout instant par la mystification. Les exigences violentes et globales qui rayaient le ciel se replient, se font modestes. Le loup impétueux qui voulait tout dévorer, la bourrasque qui voulait effectuer une authentique révolution menace, si la lutte dure, et elle dure, de devenir méconnaissable. Le colonisé risque à tout instant de se laisser désarmer par n'importe quelle concession.

Les dirigeants de l'insurrection découvrent cette instabilité du colonisé avec effroi. D'abord désorientés, ils comprennent, par ce nouveau biais, la nécessité d'expliquer et d'opérer le désenlisement radical 
de la conscience. Car la guerre dure, l'ennemi s'organise, se renforce, devine la stratégie du colonisé. La lutte de libération nationale ne consiste pas à franchir un espace d'une unique foulée. L'épopée est quotidienne, difficile, et les souffrances que l'on endure dépassent toutes celles de la période coloniale. En bas, dans les villes, il paraît que les colons ont changé. Les nôtres sont plus heureux. On les respecte. Les jours succèdent aux jours et il ne faut pas que le colonisé engagé dans la lutte, le peuple qui doit continuer à donner son appui basculent. Ils ne doivent pas s'imaginer que le but est atteint. Ils ne doivent pas, quand on leur précise les objectifs réels de la lutte, s'imaginer que cela n'est pas possible. Encore une fois, il faut expliquer, il faut que le peuple voie où il va, comment y aller. La guerre n'est pas une bataille mais une succession de combats locaux dont, à la vérité, aucun n'est décisif.

Il y a donc nécessité de ménager ses forces, de ne pas les jeter d'un seul coup dans la balance. Les réserves du colonialisme sont plus riches, plus importantes que celles du colonisé. La guerre dure. L'adversaire se défend. La grande explication [136] n'est ni pour aujourd'hui, ni pour demain. En fait, elle a commencé dès le premier jour et elle ne prendra pas fin parce qu'il n'y aura plus d'adversaire, mais tout simplement parce que ce dernier, pour de multiples raisons, se rendra compte qu'il y va de son intérêt de terminer cette lutte et de reconnaître la souveraineté du peuple colonisé. Les objectifs de la lutte ne doivent pas rester dans l'indifférenciation des premiers jours. Si l'on n'y prend garde on risque à tout instant de voir le peuple se demander, à l'occasion de la moindre concession faite par l'ennemi, les raisons de la prolongation de la guerre. On s'est à ce point habitué au mépris de l'occupant, à sa volonté affirmée de maintenir coûte que coûte son oppression que toute initiative d'allure généreuse, toute bonne disposition manifestée est saluée avec étonnement et allégresse. Le colonisé a alors tendance à chanter. Il faut multiplier les explications et faire comprendre au militant que les concessions de l'adversaire ne doivent pas l'aveugler. Ces concessions, qui ne sont rien d'autre que des concessions, ne portent pas sur l'essentiel et, dans la 
perspective du colonisé, on peut affirmer qu'une concession ne porte pas sur l'essentiel quand elle ne touche pas le régime colonial dans ce qu'il a d'essentiel.

Précisément, les formes brutales de présence de l'occupant peuvent parfaitement disparaître. En fait, cette disparition spectaculaire se révèle être un allégement des dépenses de l'occupant et une mesure positive contre l'éparpillement des forces. Mais cette disparition sera monnayée au prix fort. Très exactement au prix d'un encadrement plus coercitif du destin du pays. Des exemples historiques seront évoqués à l'aide desquels le peuple pourra se convaincre que la mascarade de la concession, que l'application du principe de la concession à tout prix se sont soldées pour certains pays par un asservissement plus discret mais plus total. Le peuple, l'ensemble des militants devront connaître cette loi historique qui stipule que certaines concessions sont, en fait, des carcans. Quand le travail de clarification n'a pas été fait, on est étonné de la facilité avec laquelle les dirigeants de certains partis politiques s'engagent dans des compromissions [137] sans nom avec l'ancien colonisateur. Le colonisé doit se persuader que le colonialisme ne lui fait aucun don. Ce que le colonisé obtient par la lutte politique ou armée n'est pas le résultat de la bonne volonté ou du bon cœur du coIon mais traduit son impossibilité à différer les concessions. Davantage, le colonisé doit savoir que ces concessions, ce n'est pas le colonialisme qui les fait, mais lui. Quand le gouvernement britannique décide d'octroyer à la population africaine quelques sièges de plus à l'Assemblée du Kenya, il faut beaucoup d'impudeur ou d'inconscience pour prétendre que le gouvernement britannique a fait des concessions. Ne voit-on pas que c'est le peuple kenyan qui fait des concessions? Il faut que les peuples colonisés, il faut que les peuples qui ont été dépouillés perdent l'attitude mentale qui jusqu'à présent les a caractérisés. À la rigueur le colonisé peut accepter un compromis avec le colonialisme mais jamais une compromission.

Toutes ces explications, ces éclairages successifs de la conscience, ce cheminement dans la voie de la connaissance de l'histoire des socié- 
tés ne sont possibles que dans le cadre d'une organisation, d'un encadrement du peuple. Cette organisation est mise sur pied par l'utilisation des éléments révolutionnaires venus des villes au début de l'insurrection et de ceux qui rejoignent les campagnes au fur et à mesure du développement de la lutte. C'est ce noyau qui constitue l'organisme politique embryonnaire de l'insurrection. Mais, de leur côté, les paysans qui élaborent leurs connaissances au contact de l'expérience se révéleront aptes à diriger la lutte populaire. Il s'installe un courant d'édification et d'enrichissement réciproque entre la nation sur le pied de guerre et ses dirigeants. Les institutions traditionnelles sont renforcées, approfondies et quelquefois littéralement transformées. Le tribunal des conflits, les djemaas, les assemblées de village se transforment en tribunal révolutionnaire, en comité politicomilitaire. Dans chaque groupe de combat, dans chaque village, surgissent des légions de commissaires politiques. Le peuple qui commence à buter sur des îlots d'incompréhension [138] sera éclairé par ces commissaires politiques. C'est ainsi que ces derniers ne craindront pas d'aborder les problèmes qui, s'ils n'étaient pas explicités, contribueraient à désorienter le peuple. Le militant en armes est, en effet, irrité de voir que beaucoup d'indigènes continuent à mener leur vie dans les cités comme s'ils étaient étrangers à ce qui se passe dans les montagnes, comme s'ils ignoraient que le mouvement essentiel est commencé. Le silence des villes, la continuation du train-train quotidien donnent au paysan l'impression amère que tout un secteur de la nation se contente de compter les points. Ces constatations révoltent les paysans et renforcent leur tendance à mépriser et à condamner globalement les urbains. Le commissaire politique devra les amener à nuancer cette position par la prise de conscience que certaines fractions de la population possèdent des intérêts particuliers qui ne recouvrent pas toujours l'intérêt national. Le peuple comprend alors que l'indépendance nationale met au jour des réalités multiples qui, quelquefois, sont divergentes et antagonistes. L'explication, à ce moment précis de la lutte, est décisive car elle fait passer le peuple du nationalisme global et indifférencié à une conscience sociale et économique. Le peuple, qui au début de la lutte avait adopté le manichéisme primitif 
du colon: les Blancs et les Noirs, les Arabes et les Roumis, s'aperçoit en cours de route qu'il arrive à des Noirs d'être plus blancs que les Blancs et que l'éventualité d'un drapeau national, la possibilité d'une nation indépendante n'entraînent pas automatiquement certaines couches de la population à renoncer à leurs privilèges ou à leurs intérêts. Le peuple s'aperçoit que des indigènes comme lui ne perdent pas le nord mais bien au contraire, semblent profiter de la guerre pour renforcer leur situation matérielle et leur puissance naissante. Les indigènes trafiquent et réalisent de véritables profits de guerre aux dépens du peuple qui, comme toujours, se sacrifie sans restrictions et arrose de son sang le sol national. Le militant qui fait face, avec des moyens rudimentaires, à la machine de guerre colonialiste se rend compte que dans le même temps où il démolit l'oppression coloniale il contribue par la bande à construire [139] un autre appareil d'exploitation. Cette découverte est désagréable, pénible et révoltante. Tout était simple pourtant, d'un côté les mauvais, de l'autre les bons. À la clarté idyllique et irréelle du début se substitue une pénombre qui disloque la conscience. Le peuple découvre que le phénomène inique de l'exploitation peut présenter une apparence noire ou arabe. Il crie à la trahison mais il faut corriger ce cri. La trahison n'est pas nationale, c'est une trahison sociale, il faut apprendre au peuple à crier au voleur. Dans son cheminement laborieux vers la connaissance rationnelle le peuple devra également abandonner le simplisme qui caractérisait sa perception du dominateur. L'espèce se morcelle devant ses yeux. Autour de lui il constate que certains colons ne participent pas à l'hystérie criminelle, qu'ils se différencient de l'espèce. Ces hommes, qu'on rejetait indifféremment dans le bloc monolithique de la présence étrangère, condamnent la guerre coloniale. Le scandale éclate vraiment quand des prototypes de cette espèce passent de l'autre côté, se font nègres ou arabes et acceptent les souffrances, la torture, la mort.

Ces exemples désarment la haine globale que le colonisé ressentait à l'égard du peuplement étranger. Le colonisé entoure ces quelques hommes d'une affection chaleureuse et a tendance, par une sorte de 
surenchère affective, à leur faire confiance de façon absolue. Dans la métropole, perçue comme marâtre implacable et sanguinaire, des voix nombreuses et quelquefois illustres prennent position, condamnent sans réserve la politique du guerre de leur gouvernement et conseillent de tenir compte enfin de la volonté nationale du peuple colonisé. Des soldats désertent les rangs colonialistes, d'autres refusent explicitement de se battre contre la liberté du peuple, vont en prison, souffrent au nom du droit de ce peuple à l'indépendance et à la gestion de ses propres affaires.

Le colon n'est plus simplement l'homme à abattre. Les membres de la masse colonialiste se révèlent être plus près, infiniment plus près de la lutte nationaliste que certains fils de la [140] nation. Le niveau racial et raciste est dépassé dans les deux sens. On ne délivre plus un brevet d'authenticité à tout nègre ou à tout musulman. On ne cherche plus son fusil ou sa machette à l'apparition de n'importe quel colon. La conscience débouche laborieusement sur des vérités partielles, limitées, instables. Tout cela, on s'en doute, est fort difficile. La tâche de rendre le peuple adulte sera facilitée à la fois par la rigueur de l'organisation et par le niveau idéologique de ses dirigeants. La puissance du niveau idéologique s'élabore et se renforce au fur et à mesure du déroulement de la lutte, des mancuvres de l'adversaire, des victoires et des revers. La direction révèle sa force et son autorité en dénonçant les erreurs, en profitant de chaque retour en arrière de la conscience pour tirer la leçon, pour assurer de nouvelles conditions de progrès. Chaque reflux local sera utilisé pour reprendre la question à l'échelle de tous les villages, de tous les réseaux. L'insurrection se prouve à elle-même sa rationalité, exprime sa maturité chaque fois qu'à partir d'un cas elle fait avancer la conscience du peuple. En dépit de l'entourage qui quelquefois incline à penser que les nuances constituent des dangers et introduisent des failles dans le bloc populaire, la direction demeure ferme sur les principes dégagés dans la lutte nationale et dans la lutte générale que l'homme mène pour sa libération. Il y a une brutalité et un mépris des subtilités et des cas individuels qui sont typiquement révolutionnaires mais il existe une autre sorte de brutali- 
té ressemblant étonnamment à la première et qui sont typiquement contre-révolutionnaires, aventurière et anarchiste. Cette brutalité pure, totale, si elle n'est pas immédiatement combattue, entraîne immanquablement la défaite du mouvement au bout de quelques semaines.

Le militant nationaliste qui avait fui la ville, ulcéré par les manœuvres démagogiques et réformistes des dirigeants, déçu par la «politique», découvre dans la praxis concrète une nouvelle politique qui ne ressemble plus du tout à l'ancienne. Cette politique est une politique de responsables, de dirigeants insérés dans l'histoire qui assument avec leurs muscles et avec leurs [141] cerveaux la direction de la lutte de libération. Cette politique et nationale, révolutionnaire, sociale. Cette nouvelle réalité que le colonisé va maintenant connaître n'existe que par l'action. C'est la lutte qui, en faisant exploser l'ancienne réalité coloniale, révèle des facettes inconnues, fait surgir des significations nouvelles et met le doigt sur les contradictions camouflées par cette réalité. Le peuple qui lutte, le peuple qui, grâce à la lutte, dispose cette nouvelle réalité et la connaît, avance, libéré du colonialisme, prévenu par avance contre toutes les tentatives de mystification, contre tous les hymnes à la nation. Seule la violence exercée par le peuple, violence organisée et éclairée par la direction, permet aux masses de déchiffrer la réalité sociale, lui en donne la clef. Sans cette lutte, sans cette connaissance dans la praxis, il n'y a plus que carnaval et flonflons. Un minimum de réadaptation, quelques réformes au sommet, un drapeau et, tout en bas, la masse indivise, toujours « moyenâgeuse », qui continue son mouvement perpétuel. 
[143]

\section{LES DAMNÉS DE LA TERRE}

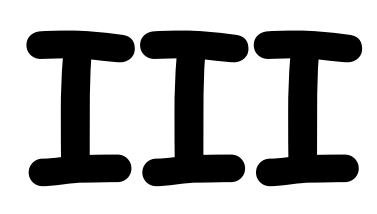

\section{MÉSAVENTURES DE LA CONSCIENCE NATIONALE}

Retour à la table des matières 
Que le combat anticolonialiste ne s'inscrive pas d'emblée dans une perspective nationaliste, c'est bien ce que l'histoire nous apprend. Pendant longtemps le colonisé dirige ses efforts vers la suppression de certaines iniquités: travail forcé, sanctions corporelles, inégalité des salaires, limitations des droits politiques, etc. Ce combat pour la démocratie contre l'oppression de l'homme va progressivement sortir de la confusion néolibérale universaliste pour déboucher, parfois laborieusement, sur la revendication nationale. Or l'impréparation des élites, l'absence de liaison organique entre elles et les masses, leur paresse et, disons-le, la lâcheté au moment décisif de la lutte vont être à l'origine de mésaventures tragiques.

La conscience nationale au lieu d'être la cristallisation coordonnée des aspirations les plus intimes de l'ensemble du peuple, au lieu d'être le produit immédiat le plus palpable de la mobilisation populaire, ne sera en tout état de cause qu'une forme sans contenu, fragile, grossière. Les failles que l'on y découvre expliquent amplement la facilité avec laquelle, dans les jeunes pays indépendants, on passe de la nation à l'ethnie, de l'État à la tribu. Ce sont ces lézardes qui rendent compte des retours en arrière, si pénibles et si préjudiciables à l'essor national, à l'unité nationale. Nous verrons que ces faiblesses et les dangers graves qu'elles renferment sont le résultat historique de l'incapacité de la bourgeoisie nationale des pays sous-développés à rationaliser la praxis populaire, c'est-à-dire à en extraire la raison. 
La faiblesse classique, quasi congénitale de la conscience nationale des pays sous-développés n'est pas seulement la [146] conséquence de la mutilation de l'homme colonisé par le régime colonial. Elle est aussi le résultat de la paresse de la bourgeoisie nationale, de son indigence, de la formation profondément cosmopolite de son esprit.

La bourgeoisie nationale qui prend le pouvoir à la fin du régime colonial est une bourgeoisie sous-développée. Sa puissance économique est presque nulle et, en tout cas, sans commune mesure avec celle de la bourgeoisie métropolitaine à laquelle elle entend se substituer. Dans son narcissisme volontariste, la bourgeoisie nationale s'est facilement convaincue qu'elle pouvait avantageusement remplacer la bourgeoisie métropolitaine. Mais l'indépendance qui la met littéralement au pied du mur va déclencher chez elle des réactions catastrophiques et l'obliger à lancer des appels angoissés en direction de l'ancienne métropole. Les cadres universitaires et commerçants qui constituent la fraction la plus éclairée du nouvel État se caractérisent en effet par leur petit nombre, leur concentration dans la capitale, le type de leurs activités: négoce, exploitations agricoles, professions libérales. Au sein de cette bourgeoisie nationale on ne trouve ni industriels, ni financiers. La bourgeoisie nationale des pays sous-développés n'est pas orientée vers la production, l'invention, la construction, le travail. Elle est tout entière canalisée vers des activités de type intermédiaire. Être dans le circuit, dans la combine, telle semble être sa vocation profonde. La bourgeoisie nationale a une psychologie d'hommes d'affaires non de capitaines d'industrie. Et il est bien vrai que la rapacité des colons et le système d'embargo installé par le colonialisme ne lui ont guère laissé le choix.

Dans le système colonial une bourgeoisie qui accumule du capital est une impossibilité. Or, précisément, il semble que la vocation historique d'une bourgeoisie nationale authentique dans un pays sous-développé soit de se nier en tant que bourgeoisie, de se nier en tant qu'instrument du capital et de se faire totalement esclave du capital révolutionnaire que constitue le peuple. 
[147]

Dans un pays sous-développé une bourgeoisie nationale authentique doit se faire un devoir impérieux de trahir la vocation à laquelle elle était destinée, de se mettre à l'école du peuple, c'est-à-dire de mettre à la disposition du peuple le capital intellectuel et technique qu'elle a arraché lors de son passage dans les universités coloniales. Nous verrons malheureusement que, assez souvent, la bourgeoisie nationale se détourne de cette voie héroïque et positive, féconde et juste, pour s'enfoncer, l'âme en paix, dans la voie horrible, parce qu'antinationale, d'une bourgeoisie classique, d'une bourgeoisie bourgeoise, platement, bêtement, cyniquement bourgeoise.

L'objectif des partis nationalistes à partir d'une certaine époque est, nous l'avons vu, strictement national. Ils mobilisent le peuple sur le mot d'ordre d'indépendance et pour le reste s'en remettent à l'avenir. Quand on interroge ces partis sur le programme économique de l'État qu'ils revendiquent, sur le régime qu'ils se proposent d'instaurer, ils se montrent incapables de répondre parce que précisément ils sont totalement ignorants à l'égard de l'économie de leur propre pays.

Cette économie s'est toujours développée en dehors d'eux. Des ressources actuelles et potentielles du sol et du sous-sol de leur pays, ils n'ont qu'une connaissance livresque, approximative. Ils ne peuvent donc en parler que sur un plan abstrait, général. Après l'indépendance cette bourgeoisie sous-développée, numériquement réduite, sans capitaux, qui refuse la voie révolutionnaire, va lamentablement stagner. Elle ne peut donner libre cours à son génie dont elle pouvait dire, un peu légèrement, qu'il était empêché par la domination coloniale. La précarité de ses moyens et la rareté de ses cadres l'acculent pendant des années à une économie de type artisanal. Dans sa perspective inévitablement très limitée, une économie nationale est une économie basée sur ce que l'on appelle les produits locaux. De grands discours seront prononcés sur l'artisanat. Dans l'impossibilité où elle se trouve de mettre en place des usines plus rentables pour le pays et pour elle, la 
bourgeoisie va entourer [148] l'artisanat d'une tendresse chauvine qui va dans le sens de la nouvelle dignité nationale et qui par ailleurs lui procurera de substantiels profits. Ce culte des produits locaux, cette impossibilité d'inventer de nouvelles directions se manifesteront également par l'enlisement de la bourgeoisie nationale dans la production agricole caractéristique de la période coloniale.

L'économie nationale de la période d'indépendance n'est pas réorientée. E s'agit toujours de récolte d'arachide, de récolte de cacao, de récolte d'olives. De même, aucune modification n'est apportée dans la traite des produits de base. Aucune industrie n'est installée dans le pays. On continue à expédier les matières premières, on continue à se faire les petits agriculteurs de l'Europe, les spécialistes de produits bruts.

Pourtant, la bourgeoisie nationale ne cesse d'exiger la nationalisation de l'économie et des secteurs commerciaux. C'est que, pour elle, nationaliser ne signifie pas mettre la totalité de l'économie au service de la nation, décider de satisfaire tous les besoins de la nation. Pour elle, nationaliser ne signifie pas ordonner l'État en fonction de rapports sociaux nouveaux dont on décide de faciliter l'éclosion. Nationalisation pour elle signifie très exactement transfert aux autochtones des passe-droits hérités de la période coloniale.

Comme la bourgeoisie n'a ni les moyens matériels, ni les moyens intellectuels suffisants (ingénieurs, techniciens), elle limitera ses prétentions à la reprise des cabinets d'affaires et des maisons de commerce autrefois occupés par les colons. La bourgeoisie nationale prend la place de l'ancien peuplement européen : médecins, avocats, commerçants, représentants, agents généraux, transitaires. Elle estime, pour la dignité du pays et sa propre sauvegarde, devoir occuper tous ces postes. Dorénavant elle va exiger que les grandes compagnies étrangères passent par elle, soit qu'elles désirent se maintenir dans le pays, soit qu'elles aient l'intention d'y pénétrer. La bourgeoise nationale se découvre la mission historique de servir d'intermédiaire. Comme on le 
voit, il ne s'agit pas d'une vocation à transformer [149] la nation, mais prosaïquement à servir de courroie de transmission à un capitalisme acculé au camouflage et qui se pare aujourd'hui du masque néocolonialiste. La bourgeoisie nationale va se complaire, sans complexes et en toute dignité, dans le rôle d'agent d'affaires de la bourgeoisie occidentale. Ce rôle lucratif, cette fonction de gagne-petit, cette étroitesse de vues, cette absence d'ambition symbolisent l'incapacité de la bourgeoisie nationale à remplir son rôle historique de bourgeoisie. L'aspect dynamique et pionnier, l'aspect inventeur et découvreur de mondes que l'on trouve chez toute bourgeoisie nationale est ici lamentablement absent. Au sein de la bourgeoisie nationale des pays coloniaux l'esprit jouisseur domine. C'est que sur le plan psychologique elle s'identifie à la bourgeoisie occidentale dont elle a sucé tous les enseignements. Elle suit la bourgeoisie occidentale dans son côté négatif et décadent sans avoir franchi les premières étapes d'exploration et d'invention qui sont en tout état de cause un acquis de cette bourgeoisie occidentale. À ses débuts la bourgeoisie nationale des pays coloniaux s'identifie à la fin de la bourgeoisie occidentale. Il ne faut pas croire qu'elle brûle les étapes. En fait elle commence par la fin. Elle est déjà sénescente alors qu'elle n'a connu ni la pétulance, ni l'intrépidité, ni le volontarisme de la jeunesse et de l'adolescence.

Dans son aspect décadent, la bourgeoisie nationale sera considérablement aidée par les bourgeoisies occidentales qui se présentent en touristes amoureux d'exotisme, de chasse, de casinos. La bourgeoisie nationale organise des centres de repos et de délassement, des cures de plaisir à l'intention de la bourgeoisie occidentale. Cette activité prendra le nom de tourisme et sera assimilée pour la circonstance à une industrie nationale. Si l'on veut une preuve de cette éventuelle transformation des éléments de la bourgeoisie ex-colonisée en organisateur de "parties» pour la bourgeoisie occidentale, il vaut la peine d'évoquer ce qui s'est passé en Amérique latine. Les casinos de La Havane, de Mexico, les plages de Rio, les petites Brésiliennes, les petites [150] Mexicaines, les métisses de treize ans, Acapulco, Copacabana 
sont les stigmates de cette dépravation de la bourgeoisie nationale. Parce qu'elle n'a pas d'idées, parce qu'elle est fermée sur elle-même, coupée du peuple, minée par son incapacité congénitale à penser l'ensemble des problèmes en fonction de la totalité de la nation, la bourgeoisie nationale va assumer le rôle de gérant des entreprises de l'Occident et pratiquement organisera son pays en lupanar de l'Europe.

Encore une fois il faut avoir devant les yeux le spectacle lamentable de certaines républiques d'Amérique latine. D'un coup d'aile les hommes d'affaires des États-Unis, les gros banquiers, les technocrates débarquent «sous les tropiques» et pendant huit à dix jours s'enfoncent dans la douce dépravation que leur offrent leurs « réserves».

Le comportement des propriétaires fonciers nationaux s'identifie pratiquement à celui de la bourgeoisie des villes. Les gros agriculteurs ont, dès la proclamation de l'indépendance, exigé la nationalisation des exploitations agricoles. À l'aide de multiples combines ils arrivent à faire main basse sur les fermes possédées autrefois par les colons, renforçant ainsi leur emprise sur la région. Mais ils n'essaient pas de renouveler l'agriculture, de l'intensifier ou de l'intégrer dans une économie réellement nationale.

En fait les propriétaires fonciers exigeront des pouvoirs publics qu'ils centuplent à leur profit les facilités et les passe-droits dont bénéficiaient autrefois les colons étrangers. L'exploitation des ouvriers agricoles sera renforcée et légitimée. Manipulant deux ou trois slogans, ces nouveaux colons vont exiger des ouvriers agricoles un travail énorme, au nom bien sûr de l'effort national. Il n'y aura pas de modernisation de l'agriculture, pas de plan de développement, pas d'initiatives, car les initiatives, qui impliquent un minimum de risques, jettent la panique dans ces milieux et mettent en déroute la bourgeoisie terrienne, hésitante, prudente, qui s'enlise de plus en plus dans les circuits mis en place par le colonialisme. Dans ces régions, les initiatives sont le fait du gouvernement. C'est le gouvernement [151] qui les 
arrête, qui les encourage, qui les finance. La bourgeoisie agricole refuse de prendre le moindre risque. Elle est rebelle au pari, à l'aventure. Elle n'entend pas travailler sur du sable. Elle exige du solide, du rapide. Les bénéfices qu'elle empoche, énormes, compte tenu du revenu national, ne sont pas réinvestis. Une épargne de bas de laine domine la psychologie de ces propriétaires fonciers. Quelquefois, surtout dans les années qui suivent l'indépendance, la bourgeoisie n'hésite pas à confier à des banques étrangères les bénéfices qu'elle tire du sol national. Par contre des sommes importantes sont utilisées en dépenses d'apparat, en voitures, en villas, toutes choses bien décrites par les économistes comme caractéristiques de la bourgeoisie sous-développée.

Nous avons dit que la bourgeoisie colonisée qui accède au pouvoir emploie son agressivité de classe à accaparer les postes anciennement détenus par les étrangers. Au lendemain de l'indépendance, en effet, elle se heurte aux séquelles humaines du colonialisme: avocats, commerçants, propriétaires terriens, médecins, fonctionnaires supérieurs. Elle va se battre impitoyablement contre ces gens «qui insultent la dignité nationale ». Elle brandit énergiquement les notions de nationalisation des cadres, d'africanisation des cadres. En fait, sa démarche va se teinter de plus en plus de racisme. Brutalement, elle pose au gouvernement un problème précis : il nous faut ces postes. Et elle ne mettra une sourdine à sa hargne que lorsqu'elle les aura totalement occupés.

De leur côté, le prolétariat des villes, la masse des chômeurs, les petits artisans, ceux que l'on a coutume d'appeler les petits métiers, se rangent sur cette attitude nationaliste, mais, rendons leur cette justice: ils ne font que calquer leur attitude sur celle de leur bourgeoisie. Si la bourgeoisie nationale entre en compétition avec les Européens, les artisans et les petits métiers déclenchent la lutte contre les Africains non nationaux. En Côte-d'Ivoire, ce sont les émeutes proprement racistes antidahoméennes et antivoltaïques. Les Dahoméens et les [152] Voltaïques qui occupaient dans le petit négoce des secteurs importants sont l'objet, au lendemain de l'indépendance, de manifesta- 
tions d'hostilité de la part des Ivoiriens. Du nationalisme nous sommes passés à l'ultra-nationalisme, au chauvinisme, au racisme. On exige le départ de ces étrangers, on brûle leurs magasins, on démolit leurs échoppes, on les lynche et, effectivement, le gouvernement ivoirien les somme de partir, donnant ainsi satisfaction aux nationaux. Au Sénégal ce sont les manifestations antisoudanaises qui feront dire à M. Mamadou Dia : «En vérité le peuple sénégalais n'a adopté la mystique du Mali que par attachement à ses leaders. Son adhésion au Mali n'a pas d'autre valeur que celle d'un nouvel acte de foi dans la politique de ces derniers. Le territoire sénégalais n'en était pas moins vivant, d'autant que la présence soudanaise à Dakar se manifestait avec trop d'indiscrétion pour le faire oublier. C'est ce fait qui explique que, loin de susciter des regrets, l'éclatement de la Fédération ait été accueilli dans les masses populaires avec soulagement et que nulle part aucun appui ne se soit manifesté pour la maintenir ${ }^{13}$.»

Tandis que certaines couches du peuple sénégalais sautent sur l'occasion qui leur est offerte par leurs propres dirigeants de se débarrasser des Soudanais qui les gênent soit dans le secteur commercial, soit dans celui de l'administration, les Congolais, qui assistaient sans y croire au départ massif des Belges, décident de faire pression sur les Sénégalais installés à Léopoldville et à Élizabethville et d'obtenir leur départ.

Comme on le voit, le mécanisme est identique dans les deux ordres de phénomènes. Si les Européens limitent la voracité des intellectuels et de la bourgeoisie d'affaires de la jeune nation, pour la masse du peuple des villes la concurrence est représentée principalement par des Africains d'une nation différente. En Côte-d'Ivoire ce sont les Dahoméens, au Ghana les Nigériens, au Sénégal, les Soudanais.

[153]

Lorsque l'exigence de négrification ou d'arabisation des cadres présentée par la bourgeoisie ne procède pas d'une entreprise authentique

13 Mamadou Dia, Nations africaines et solidarité mondiale, PUF, 1960, p. 140. 
de nationalisation mais correspond simplement au souci de confier à la bourgeoisie le pouvoir détenu jusque-là par l'étranger, les masses à leur niveau présentent la même revendication mais en restreignant aux limites territoriales la notion de nègre ou d'arabe. Entre les affirmations vibrantes sur l'unité du continent et ce comportement inspiré aux masses par les cadres, de multiples attitudes peuvent être décrites. On assiste à un va-et-vient permanent entre l'unité africaine qui sombre de plus en plus dans l'évanescence et le retour désespérant au chauvinisme le plus odieux, le plus hargneux.

«Du côté sénégalais, les leaders qui ont été les principaux théoriciens de l'unification africaine, et qui, à différentes reprises, ont sacrifié leurs organisations politiques locales et leurs positions personnelles à cette idée, portent, de bonne foi il est vrai, d'indéniables responsabilités. Leur erreur, notre erreur, a été, sous prétexte de lutte contre la balkanisation, de ne pas prendre en considération ce fait précolonial qu'est le territorialisme. Notre erreur a été de n'avoir pas, dans nos analyses, assez accordé d'attention à ce phénomène, fruit du colonialisme, mais aussi fait sociologique qu'une théorie sur l'unité, si louable ou sympathique soit-elle, ne peut abolir. Nous nous sommes laissé séduire par le mirage de la construction la plus satisfaisante pour l'esprit, et, prenant notre idéal pour une réalité, nous avons cru qu'il suffisait de condamner le territorialisme et son produit naturel, le micronationalisme, pour avoir raison d'eux et pour assurer le succès de notre entreprise chimérique ${ }^{14}$. »

Du chauvinisme sénégalais au tribalisme ouolof la distance ne saurait être grande. Et, de fait, partout où la bourgeoisie nationale par son comportement mesquin et l'imprécision de ses positions doctrinales n'a pu parvenir à éclairer l'ensemble du peuple, à poser les problèmes d'abord en fonction du peuple, [154] partout où cette bourgeoisie nationale s'est révélée incapable de dilater suffisamment sa vision du monde, on assiste à un reflux vers les positions tribalistes; on assiste, la rage au cœur, au triomphe exacerbé des ethnies. Puisque le seul mot

14 Mamadou Dia, op. cit. 
d'ordre de la bourgeoisie est : remplaçons les étrangers, et qu'elle se hâte dans tous les secteurs de se rendre justice et de prendre les places, les petits nationaux - chauffeurs de taxi, vendeurs de gâteaux, cireurs de souliers - vont également exiger que les Dahoméens rentrent chez eux ou, allant plus loin, que les Foulbés et les Peuhls retournent à leur brousse ou à leurs montagnes.

C'est dans cette perspective qu'il faut interpréter le fait que, dans les jeunes pays indépendants, triomphe çà et là le fédéralisme. La domination coloniale $a$, on le sait, privilégié certaines régions. L'économie de la colonie n'est pas intégrée à l'ensemble de la nation. Elle est toujours disposée dans des rapports de complémentarité avec les différentes métropoles. Le colonialisme n'exploite presque jamais la totalité du pays. Il se contente de mettre au jour des ressources naturelles qu'il extrait et exporte vers les industries métropolitaines, permettant ainsi une relative richesse sectorale tandis que le reste de la colonie poursuit, ou du moins approfondit, son sous-développement et sa misère.

Au lendemain de l'indépendance, les nationaux qui habitent les régions prospères prennent conscience de leur chance et par un réflexe viscéral et primaire refusent de nourrir les autres nationaux. Les régions riches en arachide, en cacao, en diamants surgissent en figure, face au panorama vide constitué par le reste de la nation. Les nationaux de ces régions regardent avec haine les autres chez qui ils découvrent envie, appétit, impulsions homicides. Les vieilles rivalités antécoloniales, les vieilles haines interethniques ressuscitent. Les Balubas refusent de nourrir les Luluas. Le Katanga se constitue en État et Albert Kalondji se fait couronner roi du sud Kasaï.

L'unité africaine, formule vague mais à laquelle les hommes et les femmes d'Afrique étaient passionnellement attachés et dont la valeur opératoire était de faire terriblement pression sur [155] le colonialisme, dévoile son vrai visage et s'émiette en régionalismes à l'intérieur d'une même réalité nationale. La bourgeoisie nationale, parce qu'elle 
est crispée sur ses intérêts immédiats, parce qu'elle ne voit pas plus loin que le bout de ses ongles, se révèle incapable de réaliser la simple unité nationale, incapable d'édifier la nation sur des bases solides et fécondes. Le front national qui avait fait reculer le colonialisme se disloque et consume sa défaite.

Cette lutte implacable que se livrent les ethnies et les tribus, ce souci agressif d'occuper les postes rendus libres par le départ de l'étranger vont également donner naissance à des compétitions religieuses. Dans les campagnes et en brousse, les petites confréries, les religions locales, les cultes maraboutiques retrouveront leur vitalité et reprendront le cycle des excommunications. Dans les grandes villes, au niveau des cadres administratifs, on assistera à la confrontation entre les deux grandes religions révélées: I'islam et le catholicisme.

Le colonialisme, qui avait tremblé sur ses bases devant la naissance de l'unité africaine, reprend ses dimensions et tente maintenant de briser cette volonté en utilisant toutes les faiblesses du mouvement. Le colonialisme va mobiliser les peuples africains en leur révélant l'existence de rivalités «spirituelles». Au Sénégal, c'est le journal Afrique nouvelle qui chaque semaine distillera la haine de l'islam et des Arabes. Les Libanais, qui possèdent sur la côte occidentale la majorité du petit commerce, sont désignés à la vindicte nationale. Les missionnaires rappellent opportunément aux masses que de grands empires noirs, bien avant l'arrivée du colonialisme européen, ont été démantelés par l'invasion arabe. On n'hésite pas à dire que c'est l'occupation arabe qui a fait le lit du colonialisme européen: on parle d'impérialisme arabe et l'on dénonce l'impérialisme culturel de l'Islam. Les musulmans sont généralement tenus à l'écart des postes de direction. Dans d'autres régions se produit le phénomène inverse et ce sont les autochtones christianisés qui sont considérés [156] comme des ennemis objectifs et conscients de l'indépendance nationale.

Le colonialisme utilise sans vergogne toutes ces ficelles, trop heureux de dresser les uns contre les autres les Africains qui hier 
s'étaient ligués contre lui. La notion de Saint-Barthélemy prend corps dans certains esprits et le colonialisme ricane doucement quand il entend les magnifiques déclarations sur l'unité africaine. À l'intérieur d'une même nation la religion morcelle le peuple et dresse les unes contre les autres les communautés spirituelles entretenues et renforcées par le colonialisme et ses instruments. Des phénomènes totalement inattendus éclatent çà et là. Dans des pays à prédominance catholique ou protestante on voit les minorités musulmanes se jeter dans une dévotion inaccoutumée. Les fêtes islamiques sont réactivées, la religion musulmane se défend pied à pied contre l'absolutisme violent de la religion catholique. On entend des ministres dire à l'adresse de tels individus que s'ils ne sont pas contents, ils n'ont qu'à aller au Caire. Quelquefois le protestantisme américain transporte sur le sol africain ses préjugés anticatholiques et entretient à travers la religion les rivalités tribales.

À l'échelle du continent, cette tension religieuse peut revêtir le visage du racisme le plus vulgaire. On divise l'Afrique en une partie blanche et une partie noire. Les appellations de substitution: Afrique au sud ou au nord du Sahara n'arrivent pas à cacher ce racisme latent. Ici, on affirme que l'Afrique blanche a une tradition de culture millénaire, qu'elle est méditerranéenne, qu'elle prolonge l'Europe, qu'elle participe de la culture gréco-latine. On regarde l'Afrique noire comme une région inerte, brutale, non civilisée... sauvage. Là, on entend à longueur de journée des réflexions odieuses sur le voile des femmes, sur la polygamie, sur le mépris supposé des Arabes pour le sexe féminin. Toutes ces réflexions rappellent par leur agressivité celles que l'on a si souvent décrites chez le colon. La bourgeoisie nationale de chacune de ces deux grandes régions, qui a assimilé jusqu'aux racines les plus pourries de la pensée colonialiste, prend le relais [157] des Européens et installe sur le continent une philosophie raciste terriblement préjudiciable pour l'avenir de l'Afrique. Par sa paresse et son mimétisme elle favorise l'implantation et le renforcement du racisme qui caractérisait l'ère coloniale. Aussi n'est-il pas étonnant, dans un pays qui se dit afri- 
cain, d'entendre des réflexions rien moins que racistes et de constater l'existence de comportements paternalistes qui laissent l'impression amère qu'on se trouve à Paris, à Bruxelles ou à Londres.

Dans certaines régions d'Afrique le paternalisme bêlant à l'égard des Noirs, l'idée obscène puisée dans la culture occidentale que le Noir est imperméable à la logique et aux sciences règnent dans leur nudité. Quelquefois on a même l'occasion de constater que les minorités noires sont confinées dans un semi-esclavage qui légitime cette espèce de circonspection, voire de méfiance, que les pays d'Afrique noire ressentent à l'égard des pays d'Afrique blanche. Il n'est pas rare qu'un citoyen d'Afrique noire se promenant dans une grande ville d'Afrique blanche s'entende traiter de "négro» par les enfants ou se voit adresser la parole en petit-nègre par des fonctionnaires.

Non, il n'est malheureusement pas exclu que des étudiants d'Afrique noire inscrits dans des collèges au nord du Sahara s'entendent demander par leurs camarades de lycée s'il existe des maisons chez eux, s'ils connaissent l'électricité, si dans leur famille ils pratiquent l'anthropophagie. Non, il n'est malheureusement pas exclu que dans certaines régions au nord du Sahara des Africains venus des pays au sud du Sahara rencontrent des nationaux qui les supplient de les emmener « n'importe où mais avec des nègres». Pareillement, dans certains jeunes États d'Afrique noire des parlementaires, voire des ministres affirment sans rire que le danger n'est point d'une réoccupation de leur pays par le colonialisme mais de l'éventuelle invasion des «Arabes vandales venus du Nord».

Comme on le voit la carence de la bourgeoisie ne se manifeste pas uniquement sur le plan économique. Parvenue au pouvoir au [158] nom d'un nationalisme étriqué, au nom de la race, la bourgeoisie, en dépit de déclarations très belles dans la forme mais totalement vides de contenu, maniant dans une complète irresponsabilité des phrases qui sortent en droite ligne des traités de morale ou de philosophie politique de 
l'Europe, va faire la preuve de son incapacité à faire triompher un catéchisme humaniste minimum. La bourgeoisie, quand elle est forte, quand elle dispose le monde en fonction de sa puissance, n'hésite pas à affirmer des idées démocratiques à prétention universalisante. Il faut à cette bourgeoisie solide économiquement des conditions exceptionnelles pour l'acculer à ne pas respecter son idéologie humaniste. La bourgeoisie occidentale, quoique fondamentalement raciste, parvient le plus souvent à masquer ce racisme en multipliant les nuances, ce qui lui permet de conserver intacte sa proclamation de l'éminente dignité humaine.

La bourgeoisie occidentale a aménagé suffisamment de barrières et de garde-fous pour ne pas craindre réellement la compétition de ceux qu'elle exploite et qu'elle méprise. Le racisme bourgeois occidental à l'égard du nègre et du "bicot» est un racisme de mépris ; c'est un racisme qui minimise. Mais l'idéologie bourgeoise, qui est proclamation d'une égalité d'essence entre les hommes, se débrouille pour rester logique avec elle-même en invitant les sous-hommes à s'humaniser à travers le type d'humanité occidental qu'elle incarne.

Le racisme de la jeune bourgeoisie nationale est un racisme de défense, un racisme basé sur la peur. Il ne diffère pas essentiellement du vulgaire tribalisme, voire des rivalités entre çofs ou confréries. On comprend que les observateurs internationaux perspicaces naient guère pris au sérieux les grandes envolées sur l'unité africaine. C'est que le nombre des lézardes perceptibles à vue d'œil est tel que l'on pressent avec suffisamment de clarté que toutes ces contradictions devront d'abord se résoudre avant que ne vienne l'heure de cette unité.

Les peuples africains se sont récemment découverts et ont décidé, au nom du continent, de peser de manière radicale sur le régime colonial. Or les bourgeoisies nationales, qui se dépêchent, [159] région après région, de constituer leur propre magot et de mettre en place un système national d'exploitation, multiplient les obstacles à la réalisation de cette «utopie». Les bourgeoisies nationales parfaitement éclairées sur leurs objectifs sont décidées à barrer la route à cette unité, à cet effort coordonné de deux cent cinquante millions 
d'hommes pour triompher à la fois de la bêtise, de la faim et de l'inhumanité. C'est pourquoi il nous faut savoir que l'unité africaine ne peut se faire que sous la poussée et sous la direction des peuples, c'est-à-dire au mépris des intérêts de la bourgeoisie.

Sur le plan intérieur et dans le cadre institutionnel, la bourgeoisie nationale va également faire la preuve de son incapacité. Dans un certain nombre de pays sous-développés le jeu parlementaire est fondamentalement faussé. Économiquement impuissante, ne pouvant mettre au jour des relations sociales cohérentes, fondées sur le principe de sa domination en tant que classe, la bourgeoisie choisit la solution qui lui semble la plus facile, celle du parti unique. Elle ne possède pas encore cette bonne conscience et cette tranquillité que seules la puissance économique et la prise en main du système étatique pourraient lui conférer. Elle ne crée pas un État qui rassure le citoyen mais qui l'inquiète.

L'État qui, par sa robustesse et en même temps sa discrétion, devrait donner confiance, désarmer, endormir, s'impose au contraire spectaculairement, s'exhibe, bouscule, brutalise, signifiant ainsi au citoyen qu'il est en danger permanent. Le parti unique est la forme moderne de la dictature bourgeoise sans masque, sans fard, sans scrupules, cynique.

Cette dictature, c'est un fait, ne va pas très loin. Elle n'arrête pas de sécréter sa propre contradiction. Comme la bourgeoisie n'a pas les moyens économiques pour assurer sa domination et distribuer quelques miettes à l'ensemble du pays, comme, par ailleurs, elle est préoccupée de se remplir les poches le plus rapidement possible, mais aussi le plus prosaiquement, le pays s'enfonce davantage dans le marasme. Et pour cacher ce [160] marasme, pour masquer cette régression, pour se rassurer et pour s'offrir des prétextes à s'enorgueillir, la bourgeoisie n'a d'autres ressources que d'élever dans la capitale des constructions grandioses, de faire ce que l'on appelle des dépenses de prestige. 
La bourgeoisie nationale tourne de plus en plus le dos à l'intérieur, aux réalités du pays en friche et regarde vers l'ancienne métropole, vers les capitalistes étrangers qui s'assurent ses services. Comme elle ne partage pas ses bénéfices avec le peuple et ne lui permet aucunement de profiter des prébendes que lui versent les grandes compagnies étrangères, elle va découvrir la nécessité d'un leader populaire auquel reviendra le double rôle de stabiliser le régime et de perpétuer la domination de la bourgeoisie. La dictature bourgeoise des pays sousdéveloppés tire sa solidité de l'existence d'un leader. Dans les pays développés, on le sait, la dictature bourgeoise est le produit de la puissance économique de la bourgeoisie. Par contre, dans les pays sousdéveloppés, le leader représente la puissance morale à l'abri de laquelle la bourgeoisie, maigre et démunie, de la jeune nation décide de s'enrichir.

Le peuple qui, des années durant, l'a vu ou entendu parler, qui de loin, dans une sorte de rêve, a suivi les démêlés du leader avec la puissance coloniale, spontanément fait confiance à ce patriote. Avant l'indépendance, le leader incarnait en général les aspirations du peuple: indépendance, libertés politiques, dignité nationale. Mais, au lendemain de l'indépendance, loin d'incarner concrètement les besoins du peuple, loin de se faire le promoteur de la dignité réelle du peuple, celle qui passe par le pain, la terre et la remise du pays entre les mains sacrées du peuple, le leader va révéler sa fonction intime : être le président général de la société de profiteurs impatients de jouir que constitue la bourgeoisie nationale.

En dépit de sa fréquente honnêteté et malgré ses déclarations sincères, le leader est objectivement le défenseur acharné des intérêts aujourd'hui conjugués de la bourgeoisie nationale et des ex-compagnies coloniales. Son honnêteté, qui est une pure [161] disposition de l'âme, s'effrite d'ailleurs progressivement. Le contact avec les masses est tellement irréel que le leader en arrive à se convaincre qu'on en veut à son autorité et qu'on met en doute les services rendus à la patrie. Le leader juge durement l'ingratitude des masses et se range chaque jour un peu plus résolument dans le camp des exploiteurs. Il se transforme 
alors, en connaissance de cause, en complice de la jeune bourgeoisie qui s'ébroue dans la corruption et la jouissance.

Les circuits économiques du jeune État s'enlisent irréversiblement dans la structure néo-colonialiste. L'économie nationale, autrefois protégée, est aujourd'hui littéralement dirigée. Le budget est alimenté par des prêts et par des dons. Tous les trimestres, les chefs d'État eux-mêmes ou les délégations gouvernementales se rendent dans les anciennes métropoles ou ailleurs, à la pêche aux capitaux.

L'ancienne puissance coloniale multiplie les exigences, accumule concessions et garanties, prenant de moins en moins de précautions pour masquer la sujétion dans laquelle elle tient le pouvoir national. Le peuple stagne lamentablement dans une misère insupportable et lentement prend conscience de la trahison inqualifiable de ses dirigeants. Cette conscience est d'autant plus aiguë que la bourgeoisie est incapable de se constituer en classe. La répartition des richesses qu'elle organise n'est pas différenciée en secteurs multiples, n'est pas étagée, ne se hiérarchise pas par demi-tons. La nouvelle caste insulte et révolte d'autant plus que l'immense majorité, les neuf dixièmes de la population continuent à mourir de faim. L'enrichissement scandaleux, rapide, impitoyable de cette caste s'accompagne d'un réveil décisif du peuple, d'une prise de conscience prometteuse de lendemains violents. La caste bourgeoise, cette partie de la nation qui annexe à son profit la totalité des richesses du pays, par une sorte de logique, inattendue d'ailleurs, va porter sur les autres nègres ou les autres Arabes des jugements péjoratifs qui rappellent à plus d'un titre la doctrine raciste des anciens représentants de la puissance coloniale. C'est à la fois la misère du peuple, l'enrichissement désordonné de la caste bourgeoise, son [162] mépris étalé pour le reste de la nation qui vont durcir les réflexions et les attitudes.

Mais les menaces qui éclosent vont entraîner le raffermissement de l'autorité et l'apparition de la dictature. Le leader, qui a derrière lui une vie de militant et de patriote dévoué, parce qu'il cautionne 
l'entreprise de cette caste et ferme les yeux sur l'insolence, la médiocrité et l'immoralité foncière de ces bourgeois, constitue un écran entre le peuple et la bourgeoisie rapace. Il contribue à freiner la prise de conscience du peuple. Il vient au secours de la caste, cache au peuple ses mancuvres devenant ainsi l'artisan le plus ardent du travail de mystification et d'engourdissement des masses. Chaque fois qu'il s'adresse au peuple il rappelle sa vie, qui fut souvent héroïque, les combats qu'il a menés au nom du peuple, les victoires qu'en son nom il a remportées, signifiant ainsi aux masses qu'elles doivent continuer à lui faire confiance. Les exemples foisonnent de patriotes africains qui ont introduit dans la lutte politique précautionneuse de leurs aînés un style décisif à caractère nationaliste. Ces hommes sont venus de la brousse. Ils ont dit, au grand scandale du dominateur et à la grande honte des nationaux de la capitale, qu'ils venaient de cette brousse et qu'ils parlaient au nom des nègres. Ces hommes, qui ont chanté la race, qui ont assumé tout le passé, l'abâtardissement et l'anthropophagie, se retrouvent aujourd'hui, hélas! à la tête d'une équipe qui tourne le dos à la brousse et qui proclame que la vocation de son peuple est de suivre, de suivre encore et toujours.

Le leader apaise le peuple. Des années après l'indépendance, incapable d'inviter le peuple à une œuvre concrète, incapable d'ouvrir réellement l'avenir au peuple, de lancer le peuple dans la voie de la construction de la nation, donc de sa propre construction, on voit le leader ressasser l'histoire de l'indépendance, rappeler l'union sacrée de la lutte de libération. Le leader, parce qu'il refuse de briser la bourgeoisie nationale, demande au peuple de refluer vers le passé et de s'enivrer de l'épopée qui a conduit à l'indépendance. Le leader - objectivement stoppe le [163] peuple et s'acharne soit à l'expulser de l'histoire, soit à l'empêcher d'y prendre pied. Pendant la lutte de libération le leader réveillait le peuple et lui promettait une marche héroïque et radicale. Aujourd'hui, il multiplie les efforts pour l'endormir et trois ou quatre fois l'an lui demande de se souvenir de l'époque coloniale et de mesurer l'immense chemin parcouru. 
Or, il faut le dire, les masses montrent une totale incapacité à apprécier le chemin parcouru. Le paysan qui continue à gratter la terre, le chômeur qui n'en finit pas de chômer n'arrivent pas, malgré les fêtes, malgré les drapeaux pourtant neufs, à se convaincre que quelque chose a vraiment changé dans leur vie. La bourgeoisie au pouvoir a beau multiplier les démonstrations, les masses ne parviennent pas à s'illusionner. Les masses ont faim et les commissaires de police aujourd'hui africains ne les rassurent pas outre mesure. Les masses commencent à bouder, à se détourner, à se désintéresser de cette nation qui ne leur fait aucune place.

De temps à autre cependant le leader se mobilise, parle à la radio, fait une tournée pour apaiser, calmer, mystifier. Le leader est d'autant plus nécessaire qu'il n'y a pas de parti. Il existait bien, pendant la période de lutte pour l'indépendance, un parti que le leader actuel a dirigé. Mais, depuis, ce parti s'est lamentablement désagrégé. Ne subsiste que le parti formel, l'appellation, l'emblème et la devise. Le parti organique, qui devait rendre possible la libre circulation d'une pensée élaborée à partir des besoins réels des masses, s'est transformé en un syndicat d'intérêts individuels. Depuis l'indépendance le parti n'aide plus le peuple à formuler ses revendications, à mieux prendre conscience de ses besoins et à mieux asseoir son pouvoir. Le parti, aujourd'hui, a pour mission de faire parvenir au peuple les instructions émanant du sommet. Il n'y a plus ce va-et-vient fécond de la base au sommet et du sommet à la base qui fonde et garantit la démocratie dans un parti. Tout au contraire, le parti s'est constitué en écran entre les masses et la direction. Il n'y a plus de vie du parti. Les cellules mises en place pendant la [164] période coloniale sont aujourd'hui dans un état de démobilisation totale.

Le militant ronge son frein. C'est alors qu'on se rend compte de la justesse des positions prises par certains militants pendant la lutte de libération. De fait, au moment du combat, plusieurs militants avaient demandé aux organismes dirigeants d'élaborer une doctrine, de préciser des objectifs, de proposer un programme. Mais, sous prétexte de 
sauvegarder l'unité nationale, les dirigeants avaient catégoriquement refusé d'aborder cette tâche. La doctrine, répétait-on, c'est l'union nationale contre le colonialisme. Et l'on allait, armé d'un slogan impétueux érigé en doctrine, toute l'activité idéologique se bornant à une suite de variantes sur le droit des peuples à disposer d'eux-mêmes, sur le vent de l'histoire qui irréversiblement emportera le colonialisme. Lorsque les militants demandaient que le vent de l'histoire soit un peu mieux analysé, les dirigeants leur opposaient l'espoir, la décolonisation nécessaire et inévitable, etc.

Après l'indépendance, le parti sombre dans une léthargie spectaculaire. On ne mobilise plus les militants qu'à l'occasion de manifestations dites populaires, de conférences internationales, des fêtes de l'indépendance. Les cadres locaux du parti sont désignés à des postes administratifs, le parti se mue en administration, les militants rentrent dans le rang et prennent le titre vide de citoyen.

Maintenant qu'ils ont rempli leur mission historique qui était d'amener la bourgeoisie au pouvoir, ils sont fermement invités à se retirer afin que la bourgeoisie puisse calmement remplir sa propre mission. Or, nous avons vu que la bourgeoisie nationale des pays sousdéveloppés est incapable de remplir une quelconque mission. Au bout de quelques années, la désagrégation du parti devient manifeste et tout observateur, même superficiel, peut se rendre compte que l'ancien parti, devenu aujourd'hui squelettique, ne sert qu'à immobiliser le peuple. Le parti, qui pendant le combat avait attiré à lui l'ensemble de la nation, se décompose. Les intellectuels, qui à la veille de l'indépendance [165] avaient rallié le parti, confirment par leur comportement actuel que ce ralliement n'avait d'autre but que de participer à la distribution du gâteau de l'indépendance. Le parti devient un moyen de réussite individuelle.

Cependant, il existe à l'intérieur du nouveau régime une inégalité dans l'enrichissement et dans l'accaparement. Certains mangent à plusieurs râteliers et se révèlent de brillants spécialistes de 
l'opportunisme. Les passe-droits se multiplient, la corruption triomphe, les mœurs se dégradent. Les corbeaux sont aujourd'hui trop nombreux et trop voraces eu égard à la maigreur du butin national. Le parti, véritable instrument du pouvoir entre les mains de la bourgeoise, renforce l'appareil d'État et précise l'encadrement du peuple, son immobilisation. Le parti aide le pouvoir à tenir le peuple. C'est, de plus en plus, un instrument de coercition et nettement antidémocratique. Le parti est objectivement, et quelquefois subjectivement, le complice de la bourgeoisie mercantile. De même que la bourgeoisie nationale escamote sa phase de construction pour se jeter dans la jouissance, pareillement, sur le plan institutionnel, elle saute la phase parlementaire et choisit une dictature de type national-socialiste. Nous savons aujourd'hui que ce fascisme à la petite semaine, qui a triomphé pendant un demi-siècle en Amérique latine, est le résultat dialectique de l'État semi-colonial de la période d'indépendance.

Dans ces pays pauvres, sous-développés, où, selon la règle, la pus grande richesse côtoie la plus grande misère, l'armée et la police constituent les piliers du régime. Une armée et une police qui, encore une règle dont il faudra se souvenir, sont conseillées par des experts étrangers. La force de cette police, la puissance de cette armée sont proportionnelles au marasme dans lequel baigne le reste de la nation. La bourgeoisie nationale se vend de plus en plus ouvertement aux grandes compagnies étrangères. À coups de prébendes, les concessions sont arrachées par l'étranger, les scandales se multiplient, les ministres [166] s'enrichissent, leurs femmes se transforment en cocottes, les députés se débrouillent et il n'est pas jusqu'à l'agent de police, jusqu'au douanier qui ne participe à cette grande caravane de la corruption.

L'opposition devient plus agressive et le peuple saisit à demi-mot sa propagande. L'hostilité à l'égard de la bourgeoisie est désormais manifeste. La jeune bourgeoisie qui semble atteinte de sénilité précoce ne tient pas compte des conseils qui lui sont prodigués et se révèle inca- 
pable de comprendre qu'il y va de son intérêt de voiler, même légèrement, son exploitation.

C'est le très chrétien journal La Semaine africaine de Brazzaville qui écrit à l'adresse des princes du régime: « Hommes en place, et vous leurs épouses, vous êtes aujourd'hui riches de votre confort, de votre instruction peut-être, de votre belle maison, de vos relations, des multiples missions qui vous sont octroyées et vous ouvrent des horizons nouveaux. Mais toute votre richesse vous fait une carapace qui vous empêche de voir la misère qui vous entoure. Prenez garde. " Cette mise en garde de La Semaine africaine adressée aux suppôts de $M$. Youlou n'a, on le devine, rien de révolutionnaire. Ce que La Semaine africaine veut signifier aux affameurs du peuple congolais, c'est que Dieu pénalisera leur conduite: "S'il n'y a pas de place dans votre cœur pour les égards vis-à-vis des gens placés en dessous de vous, il n'y aura pas de place pour vous dans la maison de Dieu. »

Il est clair que la bourgeoisie nationale ne s'inquiète guère de ces accusations. Branchée sur l'Europe, elle demeure fermement résolue à profiter de la situation. Les bénéfices énormes qu'elle retire, de l'exploitation du peuple sont exportés à l'étranger. La jeune bourgeoisie nationale est très souvent plus méfiante à l'égard du régime qu'elle a instauré que ne le sont les compagnies étrangères. Elle refuse d'investir sur le sol national et se comporte vis-à-vis de l'État qui la protège et la nourrit avec une ingratitude remarquable qu'il convient de signaler. Sur les places européennes, elle fait l'acquisition des valeurs boursières [167] étrangères et va passer le week-end à Paris ou à Hambourg. Par son comportement la bourgeoisie nationale de certains pays sous-développés rappelle les membres d'un gang qui, après chaque hold-up, dissimulent leur part aux coéquipiers et préparent sagement la retraite. Ce comportement révèle que, plus ou moins consciemment, la bourgeoisie nationale joue perdant à long terme. Elle devine que cette situation ne durera pas indéfiniment mais elle entend en profiter au maximum. Cependant une telle exploitation et une telle méfiance à l'égard de l'État déclenchent inévitablement le mécontente- 
ment au niveau des masses. C'est dans ces conditions que le régime se durcit. Alors l'armée devient le soutien indispensable d'une répression systématisée. En l'absence d'un parlement c'est l'armée qui devient l'arbitre. Mais tôt ou tard elle découvrira son importance et fera peser sur le gouvernement le risque toujours ouvert d'un pronunciamiento.

Comme on le voit, la bourgeoisie nationale de certains pays sousdéveloppés n'a rien appris dans les livres. Si elle avait mieux regardé vers les pays d'Amérique latine, elle aurait sans nul doute identifié les dangers qui la guettent. On arrive donc à la conclusion que cette micro-bourgeoisie qui fait tant de bruit est condamnée à piétiner. Dans les pays sous-développés, la phase bourgeoise est impossible. Il y aura certes une dictature policière, une caste de profiteurs, mais l'élaboration d'une société bourgeoise se révèle vouée à l'échec. Le collège des profiteurs chamarrés, qui s'arrachent les billets de banque sur le fonds d'un pays misérable, sera tôt ou tard un fétu de paille entre les mains de l'armée habilement manœuvrée par des experts étrangers. Ainsi, l'ancienne métropole pratique le gouvernement indirect, à la fois par les bourgeois qu'elle nourrit et par une armée nationale encadrée par ses experts et qui fixe le peuple, l'immobilise et le terrorise.

Les quelques remarques que nous avons pu faire sur la bourgeoisie nationale nous conduisent à une conclusion qui ne devrait pas étonner. Dans les pays sous-développés, la [168] bourgeoisie ne doit pas trouver de conditions à son existence et à son épanouissement. Autrement dit, l'effort conjugué des masses encadrées dans un parti et des intellectuels hautement conscients et armés de principes révolutionnaires doit barrer la route à cette bourgeoisie inutile et nocive.

La question théorique que l'on pose depuis une cinquantaine d'années quand on aborde l'histoire des pays sous-développés, à savoir la phase bourgeoise peut-elle ou non être sautée, doit être résolue sur le plan 
de l'action révolutionnaire et non par un raisonnement. La phase bourgeoise dans les pays sous-développés ne se justifierait que dans la mesure où la bourgeoisie nationale serait suffisamment puissante économiquement et techniquement pour édifier une société bourgeoise, créer les conditions de développement d'un prolétariat important, industrialiser l'agriculture, rendre possible enfin une authentique culture nationale.

Une bourgeoisie telle qu'elle s'est développée en Europe a pu, tout en renforçant sa propre puissance, élaborer une idéologie. Cette bourgeoisie dynamique, instruite, laïque a réussi pleinement son entreprise d'accumulation du capital et a donné à la nation un minimum de prospérité. Dans les pays sous-développés, nous avons vu qu'il n'existait pas de véritable bourgeoisie mais une sorte de petite caste aux dents longues, avide et vorace, dominée par l'esprit gagne-petit et qui s'accommode des dividendes que lui assure l'ancienne puissance coloniale. Cette bourgeoisie à la petite semaine se révèle incapable de grandes idées, d'inventivité. Elle se souvient de ce qu'elle a lu dans les manuels occidentaux et imperceptiblement elle se transforme non plus en réplique de l'Europe mais en sa caricature.

La lutte contre la bourgeoisie des pays sous-développés est loin d'être une position théorique. Il ne s'agit pas de déchiffrer la condamnation portée contre elle par le jugement de l'histoire. Il ne faut pas combattre la bourgeoisie nationale dans les pays [169] sous-développés parce qu'elle risque de freiner le développement global et harmonieux de la nation. Il faut s'opposer résolument à elle parce qu'à la lettre elle ne sert à rien. Cette bourgeoisie, médiocre dans ses gains, dans ses réalisations, dans sa pensée, tente de masquer cette médiocrité par des constructions de prestige à l'échelon individuel, par les chromes des voitures américaines, les vacances sur la Riviera, les week-ends dans les boîtes de nuit néonisées. 
Cette bourgeoisie qui se détourne de plus en plus du peuple global n'arrive même pas à arracher à l'Occident des concessions spectaculaires: investissements intéressants pour l'économie du pays, mise en place de certaines industries. Par contre les usines de montage se multiplient, consacrant ainsi le type néocolonialiste dans lequel se débat l'économie nationale. Il ne faut donc pas dire que la bourgeoisie nationale retarde l'évolution du pays, qu'elle lui fait perdre du temps ou qu'elle risque de conduire la nation dans des chemins sans issue. En fait la phase bourgeoise dans l'histoire des pays sous-développés est une phase inutile. Quand cette caste se sera anéantie, dévorée par ses propres contradictions, on s'apercevra qu'il ne s'est rien passé depuis l'indépendance, qu'il faut tout reprendre, qu'il faut repartir de zéro. La reconversion ne sera pas opérée au niveau des structures mises en place par la bourgeoisie au cours de son règne, cette caste n'ayant fait autre chose que de prendre sans changement l'héritage de l'économie, de la pensée et des institutions coloniales.

Il est d'autant plus facile de neutraliser cette classe bourgeoise qu'elle est, nous l'avons vu, numériquement, intellectuellement, économiquement faible. Dans les territoires colonisés, la caste bourgeoise après l'indépendance tire principalement sa force des accords passés avec l'ancienne puissance coloniale. La bourgeoisie nationale aura d'autant plus de chances de prendre la relève de l'oppresseur colonialiste qu'on lui aura laissé le loisir de rester en tête à tête avec l'expuissance [170] coloniale. Mais de profondes contradictions agitent les rangs de cette bourgeoisie, ce qui donne à l'observateur attentif une impression d'instabilité. Il n'y a pas encore d'homogénéité de caste. Beaucoup d'intellectuels, par exemple, condamnent ce régime basé sur la domination de quelques-uns. Dans les pays sous-développés, il existe des intellectuels, des fonctionnaires, des élites sincères qui ressentent la nécessité d'une planification de l'économie, d'une mise hors la loi des profiteurs, d'une prohibition rigoureuse de la mystification. De plus, ces hommes dans une certaine mesure luttent pour la participation massive du peuple à la gestion des affaires publiques. 
Dans les pays sous-développés qui accèdent à l'indépendance, il existe presque toujours un petit nombre d'intellectuels honnêtes, sans idées politiques bien précises, qui, instinctivement, se méfient de cette course aux postes et aux prébendes, symptomatique des lendemains de l'indépendance dans les pays colonisés. La situation particulière de ces hommes (soutien de famille nombreuse) ou leur histoire (expériences difficiles, formation morale rigoureuse) explique ce mépris si manifeste pour les débrouillards et les profiteurs. Il faut savoir utiliser ces hommes dans le combat décisif que l'on entend mener pour une orientation saine de la nation. Barrer la route à la bourgeoisie nationale, c'est, bien sûr, écarter les péripéties dramatiques des lendemains d'indépendance, les mésaventures de l'unité nationale, la dégradation des mœurs, le siège du pays par la corruption, la régression économique et, à brève échéance, un régime antidémocratique reposant sur la force et l'intimidation. Mais c'est aussi choisir le seul moyen d'avancer.

Ce qui retarde la décision et rend timides les éléments profondément démocratiques et progressistes de la jeune nation, c'est l'apparente solidité de la bourgeoisie. Dans les pays sous-développés nouvellement indépendants, au sein des villes bâties par le colonialisme grouille la totalité des cadres. L'absence d'analyse de la population globale induit les observateurs à croire à l'existence d'une bourgeoisie puissante et parfaitement [171] organisée. En fait, on le sait aujourd'hui, il n'existe pas de bourgeoisie dans les pays sous-développés. Ce qui crée la bourgeoisie, ce n'est pas l'esprit, le goût ou les manières. Ce ne sont même pas les espoirs. La bourgeoisie est avant tout le produit direct de réalités économiques précises.

Or, dans les colonies, la réalité économique est une réalité bourgeoise étrangère. À travers ses représentants, c'est la bourgeoisie métropolitaine qui se trouve présente dans les villes coloniales. La bourgeoisie aux colonies est, avant l'indépendance, une bourgeoisie occidentale, véritable succursale de la bourgeoisie métropolitaine et qui tire sa légitimité, sa force, sa stabilité de cette bourgeoisie métropolitaine. Pendant la phase d'agitation qui précède l'indépendance, des 
éléments intellectuels et commerçants indigènes, au sein de cette bourgeoisie importée, tentent de s'identifier à elle. Il existe chez les intellectuels et les commerçants indigènes, une volonté permanente d'identification avec les représentants bourgeois de la métropole.

Cette bourgeoisie qui a adopté sans réserves et dans l'enthousiasme les mécanismes de pensée caractéristiques de la métropole, qui a merveilleusement aliéné sa propre pensée et fondé sa conscience sur des bases typiquement étrangères, va s'apercevoir, la gorge sèche, qu'il lui manque cette chose qui fait une bourgeoisie, c'est-à-dire l'argent. La bourgeoisie des pays sous-développés est une bourgeoisie en esprit. Ce ne sont ni sa puissance économique, ni le dynamisme de ses cadres, ni l'envergure de ses conceptions qui lui assurent sa qualité de bourgeoisie. Aussi est-elle à ses débuts et pendant longtemps une bourgeoisie de fonctionnaires. Ce sont les postes qu'elle occupe dans la nouvelle administration nationale qui lui donneront sérénité et solidité. Si le pouvoir lui en laisse le temps et les possibilités, cette bourgeoisie arrivera à se constituer un petit bas de laine qui renforcera sa domination. Mais elle se révélera toujours incapable de donner naissance à une authentique société bourgeoise avec toutes les conséquences économiques et industrielles que cela suppose.

[172]

La bourgeoisie nationale est dès le début orientée vers des activités de type intermédiaire. La base de son pouvoir réside dans son sens du commerce et du petit négoce, dans son aptitude à rafler des commissions. Ce n'est pas son argent qui travaille mais son sens des affaires. Elle n'investit pas, elle ne peut pas réaliser cette accumulation du capital qui est nécessaire à l'éclosion et à l'épanouissement d'une bourgeoisie authentique. À cette cadence il lui faudrait des siècles pour mettre sur pied un embryon d'industrialisation. En tout état de cause elle se heurtera à l'opposition implacable de l'ancienne métropole, qui dans le cadre des conventions néo-colonialistes aura pris toutes ses précautions. 
Si le pouvoir veut sortir le pays de la stagnation et le conduire à grands pas vers le développement et le progrès, il lui faut en tout premier lieu nationaliser le secteur tertiaire. La bourgeoisie qui veut faire triompher l'esprit de lucre et de jouissance, ses attitudes méprisantes avec la masse et l'aspect scandaleux du profit, du vol devrait-on dire, investit en effet massivement dans ce secteur. Le domaine tertiaire autrefois dominé par les colons sera envahi par la jeune bourgeoisie nationale. Dans une économie coloniale le secteur tertiaire est de loin le plus important. Si l'on veut avancer on doit décider dans les premières heures de nationaliser ce secteur. Mais il est clair que cette nationalisation ne doit pas prendre l'aspect d'une étatisation rigide. Il ne s'agit pas de placer à la tête des services des citoyens non formés politiquement. Chaque fois que cette procédure a été adoptée on s'est aperçu que le pouvoir avait en fait contribué au triomphe d'une dictature de fonctionnaires formés par l'ancienne métropole qui se révélaient rapidement incapables de penser l'ensemble national. Ces fonctionnaires commencent très vite à saboter l'économie nationale, à disloquer les organismes, et la corruption, la prévarication, le détournement des stocks, le marché noir s'installent. Nationaliser le secteur tertiaire, c'est organiser démocratiquement les coopératives de vente et d'achat. C'est décentraliser ces coopératives, en intéressant les masses à la gestion des affaires publiques. Tout cela, [173] on le voit, ne peut réussir que si on politise le peuple. Auparavant on se sera rendu compte de la nécessité de clarifier une fois pour toutes un problème capital. Aujourd'hui, en effet, le principe d'une politisation des masses est généralement retenu dans les pays sous-développés. Mais il ne semble pas qu'on aborde authentiquement cette tâche primordiale. Quand on affirme la nécessité de politiser le peuple on décide de signifier dans le même temps qu'on veut être soutenu par le peuple dans l'action que l'on entreprend. Un gouvernement qui déclare vouloir politiser le peuple exprime son désir de gouverner avec le peuple et pour le peuple. Ce ne doit pas être un langage destiné à camoufler une direction bourgeoise. Les gouvernements bourgeois des pays capitalistes ont depuis longtemps dépassé cette phase infantile du pouvoir. Froidement, ils gouvernent à l'aide de leurs lois, de leur puissance économi- 
que et de leur police. Ils ne sont pas obligés, maintenant que leur pouvoir est solidement établi, de perdre leur temps en attitudes démagogiques. Ils gouvernent dans leur intérêt et ont le courage de leur pouvoir. Ils ont créé une légitimité et sont forts de leur bon droit.

La caste bourgeoise des pays nouvellement indépendants n'a encore ni le cynisme, ni la sérénité fondés sur la puissance des vieilles bourgeoisies. D'où chez elle un certain souci de cacher ses convictions profondes, de donner le change, bref de se montrer populaire. La politisation des masses n'est pas la mobilisation trois ou quatre fois l'an de dizaines ou de centaines de milliers d'hommes et de femmes. Ces meetings, ces rassemblements spectaculaires s'apparentent à la vieille tactique d'avant l'indépendance où l'on exhibait ses forces pour se prouver à soi-même et aux autres qu'on avait le peuple avec soi. La politisation des masses se propose non d'infantiliser les masses mais de les rendre adultes.

Cela nous amène à envisager le rôle du parti politique dans un pays sous-développé. Nous avons vu dans les pages précédentes que très souvent des esprits simplistes, appartenant [174] d'ailleurs à la bourgeoisie naissante, ne cessent de répéter que dans un pays sousdéveloppé la direction des affaires par un pouvoir fort, voire une dictature, est une nécessité. Dans cette perspective on charge le parti d'une mission de surveillance des masses. Le parti double l'administration et la police et contrôle les masses non pour s'assurer de leur réelle participation aux affaires de la nation mais pour leur rappeler constamment que le pouvoir attend d'elles obéissance et discipline. Cette dictature qui se croit portée par l'histoire, qui s'estime indispensable aux lendemains de l'indépendance symbolise en réalité la décision de la caste bourgeoise de diriger le pays sous-développé d'abord avec le soutien du peuple, mais bientôt contre lui. La transformation progressive du parti en un service de renseignements est l'indice que le pouvoir se tient de plus en plus sur la défensive. La mas- 
se informe du peuple est perçue comme force aveugle que l'on doit constamment tenir en laisse soit par la mystification soit par la crainte que lui inspirent les forces de police. Le parti sert de baromètre, de service de renseignements. On transforme le militant en délateur. On lui confie des missions punitives sur les villages. Les embryons de partis d'opposition sont liquidés à coups de bâton et à coups de pierres. Les candidats de l'opposition voient leurs maisons incendiées. La politique multiplie les provocations. Dans ces conditions, bien sûr, le parti est unique et $99,99 \%$ des voix reviennent au candidat gouvernemental. Nous devons dire qu'en Afrique un certain nombre de gouvernements se comportent selon ce modèle. Tous les partis d'opposition, d'ailleurs généralement progressistes, donc qui œuvraient pour une plus grande influence des masses dans la gestion des affaires publiques, qui souhaitaient une mise au pas de la bourgeoisie méprisante et mercantile, ont été par la force des matraques et des prisons condamnés au silence puis à la clandestinité.

Le parti politique dans beaucoup de régions africaines aujourd'hui indépendantes connaît une inflation terriblement grave. En présence d'un membre du parti le peuple se tait, se [175] fait mouton et publie des éloges à l'adresse du gouvernement et du leader. Mais dans la rue, le soir à l'écart du village, au café ou sur le fleuve, il faut entendre cette déception amère du peuple, ce désespoir mais aussi cette colère contenue. Le parti, au lieu de favoriser l'expression des doléances populaires, au lieu de se donner comme mission fondamentale la libre circulation des idées du peuple vers la direction, forme écran et interdit. Les dirigeants du parti se comportent comme de vulgaires adjudants et rappellent constamment au peuple qu'il faut faire «silence dans les rangs ». Ce parti qui s'affirmait le serviteur du peuple, qui prétendait travailler à l'épanouissement du peuple, dès que le pouvoir colonial lui a remis le pays, se dépêche de renvoyer le peuple dans sa caverne. Sur le plan de l'unité nationale le parti va également multiplier les erreurs. C'est ainsi que le parti dit national se comporte en parti ethnique. C'est une véritable tribu constituée en parti. Ce parti qui se proclame volon- 
tiers national, qui affirme parler au nom du peuple global, secrètement et quelquefois ouvertement organise une authentique dictature ethnique. Nous assistons non plus à une dictature bourgeoise mais à une dictature tribale. Les ministres, les chefs de cabinets, les ambassadeurs, les préfets sont choisis dans l'ethnie du leader, quelquefois même directement dans sa famille. Ces régimes de type familial semblent reprendre les vieilles lois de l'endogamie et on éprouve non de la colère mais de la honte en face de cette bêtise, de cette imposture, de cette misère intellectuelle et spirituelle. Ces chefs de gouvernement sont les véritables traîtres à l'Afrique car ils la vendent au plus terrible de ses ennemis: la bêtise. Cette tribalisation du pouvoir entraîne, on s'en doute, l'esprit régionaliste, le séparatisme. Les tendances décentralisatrices surgissent et triomphent, la nation se disloque, se démembre. Le leader qui criait: « Unité africaine » et qui pensait à sa petite famille se réveille un beau jour avec cinq tribus qui elles aussi veulent avoir leurs ambassadeurs et leurs ministres; et toujours irresponsable, toujours inconscient, toujours misérable il dénonce « la trahison».

[176]

Nous avons maintes fois signalé le rôle très souvent néfaste du leader. C'est que le parti dans certaines régions est organisé comme un gang dont le personnage le plus dur assumerait la direction. On parle volontiers de l'ascendance de ce leader, de sa force et on n'hésite pas, sur un ton complice et légèrement admiratif, à dire qu'il fait trembler ses proches collaborateurs. Pour éviter ces multiples écueils il faut se battre avec ténacité pour que jamais le parti ne devienne un instrument docile entre les mains d'un leader. Leader, du verbe anglais qui signifie conduire. Le conducteur de peuple ça n'existe plus maintenant. Les peuples ne sont plus des troupeaux et n'ont pas besoin d'être conduits. Si le leader me conduit je veux qu'il sache qu'en même temps je le conduis. La nation ne doit pas être une affaire dirigée par un manitou. Aussi comprend-on cette panique qui s'empare des sphères dirigeantes chaque fois qu'un de ces leaders tombe malade. C'est que la question qui les obsède est celle de la succession. Que deviendra le pays si le leader disparaît? Les sphères dirigeantes qui ont abdiqué 
devant le leader, irresponsables, inconscientes, préoccupées essentiellement de la bonne vie qu'elles mènent, des cocktails organisés, des voyages payés et de la rentabilité des combines, découvrent de temps à autre le vide spirituel au cour de la nation.

Un pays qui veut réellement répondre aux questions que lui pose l'histoire, qui veut développer ses villes et le cerveau de ses habitants doit posséder un parti véridique. Le parti n'est pas un instrument entre les mains du gouvernement. Bien au contraire, le parti est un instrument entre les mains du peuple. C'est lui qui arrête la politique que le gouvernement applique. Le parti n'est pas, ne doit jamais être le seul bureau politique où se retrouvent bien à leur aise tous les membres du gouvernement et les grands dignitaires du régime. Le bureau politique, trop souvent hélas, constitue tout le parti et ses membres résident en permanence dans la capitale. Dans un pays sous-développé les membres dirigeants du parti doivent fuir la capitale comme la peste. Ils doivent résider, à l'exception de quelques-uns, dans les régions rurales. On doit éviter de tout centraliser dans la grande [177] ville. Aucune excuse d'ordre administratif ne peut légitimer cette effervescence d'une capitale déjà surpeuplée et surdéveloppée par rapport aux neuf dixièmes du territoire. Le parti doit être décentralisé à l'extrême. C'est le seul moyen d'activer les régions mortes, les régions qui ne sont pas encore éveillées à la vie.

Pratiquement il y aura au moins un membre du bureau politique dans chaque région et on évitera de le nommer chef de région. Il n'aura pas entre ses mains les pouvoirs administratifs. Le membre du bureau politique régional n'est pas tenu d'occuper le plus haut rang dans l'appareil administratif régional. Il ne doit pas obligatoirement faire corps avec le pouvoir. Pour le peuple le parti n'est pas l'autorité mais l'organisme à travers lequel il exerce en tant que peuple son autorité et sa volonté. Moins il y aura de confusion, de dualité de pouvoirs, plus le parti jouera son rôle de guide et plus il constituera pour le peuple la garantie décisive. Si le parti se confond avec le pouvoir, alors être militant du parti, c'est prendre le plus court chemin pour parvenir à des fins égoïstes, 
avoir un poste dans l'administration, augmenter de grade, changer d'échelon, faire carrière.

Dans un pays sous-développé, la mise en place de directions régionales dynamiques stoppe le processus de macrocéphalisation des villes, la ruée incohérente des masses rurales vers les cités. La mise en place, dès les premiers jours de l'indépendance, de directions régionales ayant toute compétence dans une région pour la réveiller, la faire vivre, accélérer la prise de conscience des citoyens est une nécessité à laquelle un pays qui veut avancer ne saurait échapper. Sinon, autour du leader s'amassent les responsables du parti et les dignitaires du régime. Les administrations s'enflent, non parce qu'elles se développent et se différencient, mais parce que de nouveaux cousins et de nouveaux militants attendent une place et espèrent s'infiltrer dans les rouages. Et le rêve de tout citoyen est de gagner la [178] capitale, d'avoir sa part de fromage. Les localités sont désertées, les masses rurales non encadrées, non éduquées et non soutenues se détournent d'une terre mal travaillée et se dirigent vers les bourgs périphériques, enflant démesurément le lumpen-prolétariat.

L'heure d'une nouvelle crise nationale n'est pas loin. Nous pensons au contraire que l'intérieur, l'arrière-pays devrait être privilégié. À l'extrême d'ailleurs, il n'y aurait aucun inconvénient à ce que le gouvernement siège ailleurs que dans la capitale. Il faut désacraliser la capitale et montrer aux masses déshéritées que c'est pour elles que l'on décide de travailler. C'est dans un certain sens ce que le gouvernement brésilien a tenté de faire avec Brasilia. La morgue de Rio de Janeiro était une insulte pour le peuple brésilien. Mais malheureusement Brasilia est encore une nouvelle capitale aussi monstrueuse que la première. Le seul intérêt de cette réalisation est qu'aujourd'hui il existe une route à travers la brousse. Non, aucun motif sérieux ne peut s'opposer au choix d'une autre capitale, au déplacement de l'ensemble du gouvernement vers l'une des régions les plus démunies. La capitale des pays 
sous-développés est une notion commerciale héritée de la période coloniale. Mais dans les pays sous-développés, nous devons multiplier les contacts avec les masses rurales. Nous devons faire une politique nationale, c'est-à-dire avant tout une politique pour les masses. Nous ne devons jamais perdre le contact avec le peuple qui a lutté pour son indépendance et l'amélioration concrète de son existence.

Les fonctionnaires et les techniciens autochtones doivent s'enfoncer non dans les diagrammes et les statistiques, mais dans le corps du peuple. Ils ne doivent plus se hérisser chaque fois qu'il est question d'un déplacement vers «l'intérieur». On ne doit plus voir ces jeunes femmes de pays sous-développés menacer leurs maris de divorce si jamais ils ne se débrouillent pas pour éviter l'affectation dans un poste rural. C'est pourquoi [179] le bureau politique du parti doit privilégier les régions déshéritées, et la vie de la capitale, vie factice, superficielle, plaquée sur la réalité nationale comme un corps étranger, doit tenir le moins de place possible dans la vie de la nation qui, elle, est fondamentale et sacrée.

Dans un pays sous-développé, le parti doit être organisé de telle sorte qu'il ne se contente pas d'avoir des contacts avec les masses. Le parti doit être l'expression directe des masses. Le parti n'est pas une administration chargée de transmettre les ordres du gouvernement. Il est le porte-parole énergique et le défenseur incorruptible des masses. Pour parvenir à cette conception du parti, il faut avant toute chose se débarrasser de l'idée très occidentale, très bourgeoise donc très méprisante que les masses sont incapables de se diriger. L'expérience prouve, en fait, que les masses comprennent parfaitement les problèmes les plus compliqués. Ce sera l'un des plus grands services que la révolution algérienne aura rendus aux intellectuels algériens que de les avoir mis en contact avec le peuple, de leur avoir permis de voir l'extrême, l'ineffable misère du peuple et en même temps d'assister à l'éveil de son intelligence, aux progrès de sa conscience. Le peuple algérien, cette masse d'affamés et d'analphabètes, ces hommes et ces femmes plongés pendant des siècles dans 
l'obscurité la plus effarante ont tenu contre les chars et les avions, contre le napalm et les services psychologiques, mais surtout contre la corruption et le lavage de cerveau, contre les traîtres et les armées «nationales» du général Bellounis. Ce peuple a tenu malgré les faibles, les hésitants, les apprentis dictateurs. Ce peuple a tenu parce que pendant sept ans sa lutte lui a ouvert des domaines dont il ne soupçonnait même pas l'existence. Aujourd'hui, des armureries fonctionnent en plein djebel à plusieurs mètres sous terre, aujourd'hui, des tribunaux du peuple fonctionnent à tous les échelons, des commissions locales de planification organisent le démembrement des grandes propriétés, élaborent l'Algérie de demain. Un homme isolé peut se montrer rebelle à la compréhension d'un problème mais le groupe, le village comprend avec une rapidité [180] déconcertante. Il est vrai que si l'on prend la précaution d'utiliser un langage compréhensible par les seuls licenciés en droit ou en sciences économiques, la preuve sera aisément faite que les masses doivent être dirigées. Mais si l'on parle le langage concret, si l'on n'est pas obsédé par la volonté perverse de brouiller les cartes, de se débarrasser du peuple, alors on s'aperçoit que les masses saisissent toutes les nuances, toutes les astuces. Le recours à un langage technique signifie que l'on est décidé à considérer les masses comme des profanes. Ce langage dissimule mal le désir des conférenciers de tromper le peuple, de le laisser en dehors. L'entreprise d'obscurcissement du langage est un masque derrière lequel se profile une plus vaste entreprise de dépouillement. On veut à la fois enlever au peuple et ses biens et sa souveraineté. On peut tout expliquer au peuple à condition toutefois qu'on veuille vraiment qu'il comprenne. Et, si l'on pense qu'on n'a pas besoin de lui, qu'au contraire il risque de gêner la bonne marche des multiples sociétés privées et à responsabilité limitée, dont le but est de rendre le peuple plus misérable encore, alors la question est tranchée.

Si l'on pense qu'on peut parfaitement diriger un pays sans que le peuple y mette le nez, si l'on pense que le peuple par sa seule présence trouble le jeu, soit qu'il le retarde, soit que par sa naturelle incons- 
cience il le sabote, alors aucune hésitation n'est permise : il faut écarter le peuple. Or, il se trouve que le peuple, quand on l'invite à la direction du pays, ne retarde pas mais accélère le mouvement. Nous, Algériens, au cours de cette guerre avons eu l'occasion, le bonheur de toucher du doigt un certain nombre de choses. Dans certaines régions rurales, les responsables politico-militaires de la révolution se sont en effet trouvés confrontés à des situations qui ont exigé des solutions radicales. Nous aborderons quelques-unes de ces situations.

Au cours des années 1956-1597, le colonialisme français avait interdit certaines zones, et la circulation des personnes dans ces régions était strictement réglementée. Les paysans [181] n'avaient donc plus la possibilité de se rendre librement à la ville et de renouveler leurs provisions. Les épiciers pendant cette période accumulaient des bénéfices énormes. Le thé, le café, le sucre, le tabac, le sel atteignaient des prix exorbitants. Le marché noir triomphait avec une singulière insolence. Les paysans qui ne pouvaient pas payer en espèces hypothéquaient leurs récoltes, voire leurs terres, ou démembraient lopin par lopin le patrimoine familial et dans une deuxième phase y travaillaient pour le compte de l'épicier. Les commissaires politiques dès qu'ils eurent pris conscience de ce danger réagirent de manière immédiate. C'est ainsi qu'un système rationnel d'approvisionnement fut institué : l'épicier qui se rend à la ville est tenu de faire ses achats chez des grossistes nationalistes qui lui remettent une facture où sont précisés les prix des marchandises. Quand le détaillant arrive dans le douar, il doit avant toute chose se présenter au commissaire politique qui contrôle la facture, fixe la marge bénéficiaire et arrête le prix de vente. Les prix imposés sont affichés dans la boutique et un membre du douar, sorte de contrôleur, est présent qui renseigne le fellah sur les prix auxquels doivent être vendus les produits. Mais le détaillant découvre rapidement une astuce et après trois ou quatre jours déclare son stock épuisé. Par en dessous, il reprend son trafic et continue la vente au marché noir. La réaction de l'autorité politico-militaire fut radicale. Des pénalisations importantes furent décidées, les amendes 
récoltées et versées dans la caisse du village servirent soit à des œuvres sociales, soit à des travaux d'intérêt collectif. Quelquefois, on décida de fermer pour un certain temps le commerce. Puis en cas de récidive le fonds de commerce est immédiatement saisi et un comité de gestion élu l'exploite, quitte à verser à l'ancien propriétaire une mensualité.

À partir de ces expériences, on expliqua au peuple le fonctionnement des grandes lois économiques en se basant sur des cas concrets. L'accumulation du capital cessa d'être une théorie pour devenir un comportement très réel et très présent. Le peuple comprit comment à partir d'un commerce on peut [182] s'enrichir, agrandir ce commerce. Alors, seulement, les paysans racontèrent que cet épicier leur prêtait à des taux usuraires : d'autres rappelèrent comment il les avait expulsés de leurs terres et comment de propriétaires ils étaient devenus ouvriers. Plus le peuple comprend, plus il devient vigilant, plus il devient conscient qu'en définitive tout dépend de lui et que son salut réside dans sa cohésion, dans la connaissance de ses intérêts et de l'identification de ses ennemis. Le peuple comprend que la richesse n'est pas le fruit du travail mais le résultat d'un vol organisé et protégé. Les riches cessent d'être des hommes respectables, ils ne sont plus que des bêtes carnassières, des chacals et des corbeaux qui se vautrent dans le sang du peuple. Dans une autre perspective les commissaires politiques ont dû décider que personne ne travaillerait plus pour personne. La terre est à ceux qui la travaillent. C'est un principe qui est devenu par l'explication une loi fondamentale au sein de la révolution algérienne. Les paysans qui employaient des ouvriers agricoles ont été obligés de remettre des parts à leurs anciens employés.

Alors, on s'est aperçu que le rendement à l'hectare triplait et cela malgré les raids nombreux des Français, les bombardements aériens et la difficulté d'approvisionnement en engrais. Les fellahs qui, au moment de la récolte, pouvaient apprécier et peser les produits obtenus, ont voulu comprendre ce phénomène. Ils ont très facilement découvert que le travail n'est pas une notion simple, que l'esclavage ne permet pas le 
travail, que le travail suppose la liberté, la responsabilité et la conscience.

Dans ces régions où nous avons pu mener à bien ces expériences édifiantes, où nous avons assisté à la construction de l'homme par l'institution révolutionnaire, les paysans ont saisi très clairement ce principe qui veut qu'on travaille avec d'autant plus de goût qu'on s'engage plus lucidement dans l'effort. On a pu faire comprendre aux masses que le travail n'est pas une dépense d'énergie, ou le fonctionnement de certains muscles, mais qu'on travaille davantage avec son cerveau et son cœur [183] qu'avec ses muscles et sa sueur. De même, dans ces régions libérées mais en même temps exclues de l'ancien circuit commercial on a dû modifier la production autrefois uniquement tournée vers les villes et l'exportation. On a mis en place une production de consommation pour le peuple et pour les unités de l'Armée de libération nationale. On a quadruplé la production de lentilles et organisé la fabrication du charbon de bois. Les légumes verts et le charbon ont été dirigés des régions du nord vers le sud par les montagnes, tandis que les zones du sud envoyaient de la viande vers le nord. C'est le FLN qui a décidé cette coordination, qui a mis en place le système de communications. Nous n'avions pas de techniciens, pas de planificateurs venus des grandes écoles occidentales. Mais dans ces régions libérées, la ration journalière atteignait le chiffre inconnu jusqu'alors de 3200 calories. Le peuple ne s'est pas contenté de triompher de cette épreuve. Il s'est posé des questions théoriques. Par exemple: pourquoi certaines régions ne voyaient-elles jamais d'oranges avant la guerre de libération, alors qu'on en expédiait annuellement des milliers de tonnes vers l'étranger, pourquoi les raisins étaient-ils inconnus d'un grand nombre d'Algériens alors que des millions de grappes faisaient les délices des peuples européens? Le peuple a aujourd'hui une notion très claire de ce qui lui appartient. Le peuple algérien sait aujourd'hui qu'il est le propriétaire exclusif du sol et du sous-sol de son pays. Et si certains ne comprennent pas l'acharnement du FLN à ne tolérer aucun empiétement sur cette propriété et sa farouche volonté de refuser 
tout compromis sur les principes, alors il faut que les uns et les autres se souviennent que le peuple algérien est aujourd'hui un peuple adulte, responsable, conscient. En bref, le peuple algérien est un peuple propriétaire.

Si nous avons pris l'exemple algérien pour éclairer notre propos, ce n'est point pour magnifier notre peuple, mais tout simplement pour montrer l'importance qu'a jouée le combat qu'il a mené dans sa prise de conscience. Il est clair que d'autres peuples sont arrivés au même résultat par des voies différentes. [184] En Algérie, on le sait mieux aujourd'hui, l'épreuve de force n'était pas évitable mais d'autres régions ont, par la lutte politique et le travail de clarification entrepris par le parti, conduit leurs peuples aux mêmes résultats. En Algérie, nous avons compris que les masses sont à la hauteur des problèmes auxquels elles sont confrontées. Dans un pays sous-développé, l'expérience prouve que l'important n'est pas que trois cents personnes conçoivent et décident mais que l'ensemble, même au prix d'un temps double ou triple, comprenne et décide. En fait le temps mis à expliquer, le temps «perdu » à humaniser le travailleur sera rattrapé dans l'exécution. Les gens doivent savoir où ils vont et pourquoi ils y vont. L'homme politique ne doit pas ignorer que l'avenir restera bouché tant que la conscience du peuple sera rudimentaire, primaire, opaque. Nous, hommes politiques africains, devons avoir des idées très claires sur la situation de notre peuple. Mais cette lucidité doit demeurer profondément dialectique. Le réveil du peuple global ne se fera pas d'un seul coup, son engagement rationnel dans l'œuvre d'édification nationale sera linéaire, d'abord parce que les voies de communications et les moyens de transmission sont peu développés, ensuite parce que la temporalité doit cesser d'être celle de l'instant ou de la prochaine récolte pour devenir celle du monde, enfin parce que le découragement installé très profondément dans le cerveau par la domination coloniale est toujours à fleur de peau. Mais nous ne devons pas ignorer que la victoire sur les nœuds de moindre résistance, héritages de la domination matérielle et spirituelle du pays, est une nécessité à laquelle aucun gouvernement ne 
saurait échapper. Prenons l'exemple du travail en régime colonial. Le colon n'a cessé d'affirmer que l'indigène est lent. Aujourd'hui, dans certains pays indépendants, on entend des cadres reprendre cette condamnation. En vérité, le colon voulait que l'esclave fût enthousiaste. Il voulait, par une sorte de mystification qui constitue l'aliénation la plus sublime, persuader l'esclave que la terre qu'il travaille est à lui, que les mines où il perd sa santé sont sa propriété. Le colon oubliait singulièrement qu'il s'enrichissait de l'agonie de [185] l'esclave. Pratiquement le colon disait au colonisé: «Crève, mais que je m'enrichisse. » Aujourd'hui, nous devons procéder différemment. Nous ne devons pas dire au peuple: «Crève, mais que le pays s'enrichisse. » Si nous voulons augmenter le revenu national, diminuer l'importation de certains produits inutiles, voire nocifs, augmenter la production agricole et lutter contre l'analphabétisme, il nous faut expliquer. Il faut que le peuple comprenne l'importance de l'enjeu. La chose publique doit être la chose du public. On débouche donc sur la nécessité de multiplier les cellules à la base. Trop souvent en effet, on se contente d'installer des organismes nationaux au sommet et toujours dans la capitale: I'Union des femmes, I'Union des jeunes, les syndicats, etc. Mais si l'on s'avise de chercher derrière le bureau installé dans la capitale, si l'on passe dans l'arrière-salle où devraient se trouver les archives, on est effaré par le vide, par le néant, par le bluff. Il faut une base, des cellules qui donnent précisément contenu et dynamisme. Les masses doivent pouvoir se réunir, discuter, proposer, recevoir des instructions. Les citoyens doivent avoir la possibilité de parler, de s'exprimer, d'inventer. La réunion de cellule, la réunion du comité est un acte liturgique. C'est une occasion privilégiée qui est donnée à l'homme d'écouter et de dire. À chaque réunion, le cerveau multiplie ses voies d'association, l'œil découvre un panorama de plus en plus humanisé.

La forte proportion de jeunes dans les pays sous-développés pose au gouvernement des problèmes spécifiques qu'il importe d'aborder lucidement. La jeunesse urbaine inactive et souvent illettrée est livrée 
à toutes sortes d'expériences dissolvantes. À la jeunesse sousdéveloppée sont le plus souvent offerts des jeux de pays industrialisés. Normalement en effet, il y a homogénéité entre le niveau mental et matériel des membres d'une société et les plaisirs que cette société se donne. Or, dans les pays sous-développés la jeunesse dispose de jeux pensés pour la jeunesse des pays capitalistes: romans policiers, machines à sous, photographies obscènes, littérature pornographique, [186] films-interdits-aux-moins-de-seize-ans, et surtout l'alcool... En Occident, le cadre familial, la scolarisation, le niveau de vie relativement élevé des masses laborieuses servent de rempart relatif à l'action néfaste de ces jeux. Mais dans un pays africain où le développement mental est inégal, où le heurt violent de deux mondes a ébranlé considérablement les vieilles traditions et disloqué l'univers de la perception, l'affectivité du jeune Africain, sa sensibilité sont à la merci des différentes agressions contenues dans la culture occidentale. Sa famille se révèle très souvent incapable d'opposer à ces violences la stabilité, l'homogénéité.

Dans ce domaine, le gouvernement doit servir de filtre et de stabilisateur. Les commissaires à la jeunesse des pays sous-développés commettent fréquemment une erreur. Ils conçoivent leur rôle à la manière des commissaires à la jeunesse des pays développés. Ils parlent de fortifier l'âme, d'épanouir le corps, de faciliter la manifestation de qualités sportives. Ils doivent à notre avis se garder de cette conception. La jeunesse d'un pays sous-développé est souvent une jeunesse désceuvrée. Il faut d'abord l'occuper. C'est pourquoi le commissaire à la jeunesse doit être institutionnellement rattaché au ministère du Travail. Le ministère du Travail, qui est une nécessité dans un pays sousdéveloppé, fonctionne en étroite collaboration avec le ministère du Plan, autre nécessité dans un pays sous-développé. La jeunesse africaine ne doit pas être dirigée vers les stades mais vers les champs, vers les champs et vers les écoles. Le stade n'est pas ce lieu d'exhibition installé dans les villes mais un certain espace au sein des terres que l'on défriche, que l'on travaille et que l'on offre à la nation. La concep- 
tion capitaliste du sport est fondamentalement différente de celle qui devrait exister en pays sous-développé. L'homme politique africain ne doit pas se préoccuper de faire des sportifs mais des hommes conscients qui, par ailleurs, sont sportifs. Si le sport n'est pas intégré dans la vie nationale, c'est-à-dire dans la construction nationale, si l'on construit des sportifs nationaux et non des hommes conscients, alors rapidement on assistera au pourrissement du [187] sport par le professionnalisme, le commercialisme. Le sport ne doit pas être un jeu, une distraction que s'offre la bourgeoisie des villes. La plus grande tâche est de comprendre à tout instant ce qui se passe chez nous. Nous ne devons pas cultiver l'exceptionnel, chercher le héros, autre forme du leader. Nous devons soulever le peuple, agrandir le cerveau du peuple, le meubler, le différencier, le rendre humain.

Nous retombons encore dans cette obsession que nous voudrions voir partagée par l'ensemble des hommes politiques africains, de la nécessité d'éclairer l'effort populaire, d'illuminer le travail, de le débarrasser de son opacité historique. Être responsable dans un pays sous-développé, c'est savoir que tout repose en définitive sur l'éducation des masses, sur l'élévation de la pensée, sur ce qu'on appelle trop rapidement la politisation.

On croit souvent en effet, avec une légèreté criminelle, que politiser les masses c'est épisodiquement leur tenir un grand discours politique. On pense qu'il suffit au leader ou à un dirigeant de parler sur un ton doctoral des grandes choses de l'actualité pour être quitte avec cet impérieux devoir de politisation des masses. Or, politiser c'est ouvrir l'esprit, c'est éveiller l'esprit, mettre au monde l'esprit. C'est, comme le disait Césaire, "inventer des âmes ». Politiser les masses ce n'est pas, ce ne peut pas être faire un discours politique. C'est s'acharner avec rage à faire comprendre aux masses que tout dépend d'elles, que si nous stagnons c'est de leur faute et que si nous avançons, c'est aussi de leur faute, qu'il n'y a pas de démiurge, qu'il n'y a pas d'homme illustre et responsable de tout, mais que le démiurge c'est le peuple et que les mains magiciennes ne sont en définitive que les mains 
du peuple. Pour réaliser ces choses, pour les incarner véritablement, répétons-le, il faut décentraliser à l'extrême. La circulation du sommet à la base et de la base au sommet doit être un principe rigide non par souci de formalisme mais parce que tout simplement le respect de ce principe est la garantie du salut. C'est de la base que montent les forces qui dynamisent le sommet et lui permettent dialectiquement [188] d'effectuer un nouveau bond. Encore une fois nous, Algériens, avons compris très rapidement ces choses car aucun membre d'aucun sommet n'a eu la possibilité de se prévaloir d'une quelconque mission de salut. C'est la base qui se bat en Algérie et cette base n'ignore pas que sans son combat quotidien, héroïque et difficile, le sommet ne tiendrait pas. Comme elle sait que sans un sommet et sans une direction la base éclaterait dans l'incohérence et l'anarchie. Le sommet ne tire sa valeur et sa solidité que de l'existence du peuple au combat. À la lettre, c'est le peuple qui se donne librement un sommet et non le sommet qui tolère le peuple.

Les masses doivent savoir que le gouvernement et le parti sont à leur service. Un peuple digne, c'est-à-dire conscient de sa dignité, est un peuple qui n'oublie jamais ces évidences. Pendant l'occupation coloniale on a dit au peuple qu'il fallait qu'il donne sa vie pour le triomphe de la dignité. Mais les peuples africains ont vite compris que leur dignité n'était pas seulement contestée par l'occupant. Les peuples africains ont rapidement compris qu'il y avait une équivalence absolue entre la dignité et la souveraineté. En fait, un peuple digne et libre est un peuple souverain. Un peuple digne est un peuple responsable. Et il ne sert à rien de «montrer » que les peuples africains sont infantiles ou débiles. Un gouvernement et un parti ont le peuple qu'ils méritent. Et à plus ou moins longue échéance un peuple a le gouvernement qu'il mérite.

L'expérience concrète dans certaines régions vérifie ces positions. Au cours de réunions, il arrive parfois que des militants se réfèrent pour résoudre les problèmes difficiles à la formule : «il n'y a qu'à... ». 
Ce raccourci volontariste où culminent dangereusement spontanéité, syncrétisme simplifiant, non-élaboration intellectuelle triomphe fréquemment. Chaque fois qu'on rencontre cette abdication de la responsabilité chez un militant il ne suffit pas de lui dire qu'il a tort. Il faut le rendre responsable, l'inviter à aller jusqu'au bout de son raisonnement et lui faire [189] toucher le caractère souvent atroce, inhumain et en définitive stérile de ce «il n'y a qu'à». Personne ne détient la vérité, ni le dirigeant, ni le militant. La recherche de la vérité dans des situations locales est affaire collective. Certains ont une expérience plus riche, élaborent plus rapidement leur pensée, ont pu établir dans le passé un plus grand nombre de liaisons mentales. Mais ils doivent éviter d'écraser le peuple car le succès de la décision adoptée dépend de l'engagement coordonné et conscient de l'ensemble du peuple. Personne ne peut retirer son épingle du jeu. Tout le monde sera abattu ou torturé et, dans le cadre de la nation indépendante, tout le monde aura faim et participera au marasme. Le combat collectif suppose une responsabilité collective à la base et une responsabilité collégiale au sommet. Oui, il faut compromettre tout le monde dans le combat pour le salut commun. Il n'y a pas de mains pures, il n'y a pas d'innocents, pas de spectateurs. Nous sommes tous en train de nous salir les mains dans les marais de notre sol et le vide effroyable de nos cerveaux. Tout spectateur est un lâche ou un traître.

Le devoir d'une direction est d'avoir les masses avec elle. Or, l'adhésion suppose la conscience, la compréhension de la mission à remplir, bref une intellectualisation même embryonnaire. On ne doit pas envoûter le peuple, le dissoudre dans l'émotion et dans la confusion. Seuls des pays sous-développés dirigés par des élites révolutionnaires surgies du peuple peuvent aujourd'hui permettre l'accession des masses sur la scène de l'histoire. Mais, encore une fois, faut-il que nous nous opposions vigoureusement et définitivement à la naissance d'une bourgeoisie nationale, d'une caste de privilégiés. Politiser les masses, c'est rendre la nation globale présente à chaque citoyen. C'est faire de l'expérience de la nation l'expérience de chaque citoyen. Comme le rap- 
pelait si opportunément le président Sékou Touré dans son message au deuxième Congrès des écrivains africains: «Dans le domaine de la pensée, l'homme peut prétendre à être le cerveau du monde, mais sur le plan de la vie concrète où toute intervention affecte l'être physique et spirituel, [190] le monde est toujours le cerveau de l'homme, car c'est à ce niveau que se trouvent la totalisation des puissances et des unités pensantes, les forces dynamiques de développement et de perfectionnement, c'est là que s'opère la fusion des énergies et que s'inscrit en définitive la somme des valeurs intellectuelles de l'homme. » L'expérience individuelle parce qu'elle est nationale, maillon de l'existence nationale cesse d'être individuelle, limitée, rétrécie et peut déboucher sur la vérité de la nation et du monde. De même que lors de la phase de lutte chaque combattant tenait la nation à bout de bras, de même pendant la phase de construction nationale chaque citoyen doit-il continuer dans son action concrète de tous les jours à s'associer à l'ensemble de la nation, à incarner la vérité constamment dialectique de la nation, à vouloir ici et maintenant le triomphe de l'homme total. Si la construction d'un pont ne doit pas enrichir la conscience de ceux qui y travaillent, que le pont ne soit pas construit, que les citoyens continuent de traverser le fleuve à la nage ou par bac. Le pont ne doit pas être parachuté, il ne doit pas être imposé par un deus ex machina au panorama social, mais il doit au contraire sortir des muscles et du cerveau des citoyens. Et, certes, il faudra peut-être des ingénieurs et des architectes, quelquefois entièrement étrangers, mais les responsables locaux du parti doivent être présents pour que la technique s'infiltre dans le désert cérébral du citoyen, pour que le pont dans ses détails et dans son ensemble soit repris, conçu et assumé. Il faut que le citoyen s'approprie le pont. Alors seulement tout est possible.

Un gouvernement qui se proclame national doit assumer l'ensemble de la nation et, dans les pays sous-développés, la jeunesse représente l'un des secteurs les plus importants. Il faut élever la conscience des jeunes, l'éclairer. C'est cette jeunesse que nous retrouverons dans l'armée nationale. Si le travail d'explication a été fait au niveau des 
jeunes, si l'Union nationale des jeunes a rempli sa tâche qui est d'intégrer la jeunesse dans la nation, alors pourront être évitées les erreurs qui ont hypothéqué, voire miné, l'avenir des républiques d'Amérique latine. L'armée n'est jamais une école de guerre mais une école de [191] civisme, une école politique. Le soldat d'une nation adulte n'est pas un mercenaire mais un citoyen qui par le moyen des armes défend la nation. C'est pourquoi il est fondamental que le soldat sache qu'il est au service du pays et non d'un officier aussi prestigieux soit-il. Il faut profiter du service national civil et militaire pour élever le niveau de la conscience nationale, pour détribaliser, unifier. Dans un pays sous-développé on s'efforcera le plus rapidement possible de mobiliser les hommes et les femmes. Le pays sous-développé doit se garder de perpétuer les traditions féodales qui consacrent la priorité de l'élément masculin sur l'élément féminin. Les femmes recevront une place identique aux hommes non dans les articles de la Constitution mais dans la vie quotidienne, à l'usine, à l'école, dans les assemblées. Si dans les pays occidentaux on encaserne les militaires, cela ne veut pas dire que ce soit toujours la meilleure formule. On n'est pas tenu de militariser les recrues. Le service peut être civil ou militaire et de toute façon il est recommandé que chaque citoyen valide puisse à tout moment s'intégrer dans une unité combattante et défendre les acquisitions nationales et sociales.

Les grands travaux d'intérêt collectif doivent pouvoir être exécutés par les recrues. C'est un moyen prodigieux d'activer les régions inertes, de faire connaître à un plus grand nombre de citoyens les réalités du pays. Il faut éviter de transformer l'armée en un corps autonome qui tôt ou tard, désœuvré et sans mission, se mettra à " faire de la politique » et à menacer le pouvoir. Les généraux de salon, à force de fréquenter les antichambres du pouvoir, rêvent de pronunciamientos. Le seul moyen d'y échapper est de politiser l'armée, c'est-à-dire de la nationaliser. De même y a-t-il urgence à multiplier les milices. En cas de guerre, c'est la nation entière qui se bat ou qui travaille. Il ne doit pas y avoir de soldats de métier et le nombre d'officiers de car- 
rière doit être réduit au minimum. D'abord parce que très souvent les officiers sont choisis au sein des cadres universitaires qui pourraient être beaucoup plus utiles ailleurs: un ingénieur est mille fois plus indispensable à la nation qu'un officier. [192] Ensuite, parce qu'il faut éviter la cristallisation d'un esprit de caste. Nous avons vu dans les pages précédentes que le nationalisme, ce chant magnifique qui soulevait les masses contre l'oppresseur, se désagrège au lendemain de l'indépendance. Le nationalisme n'est pas une doctrine politique, n'est pas un programme. Si l'on veut vraiment éviter à son pays ces retours en arrière, ces arrêts, ces failles, il faut rapidement passer de la conscience nationale à la conscience politique et sociale. La nation n'existe nulle part si ce n'est dans un programme élaboré par une direction révolutionnaire et repris lucidement et avec enthousiasme par les masses. Il faut situer constamment l'effort national dans le cadre général des pays sous-développés. Le front de la faim et de l'obscurité, le front de la misère et de la conscience embryonnaire doit être présent à l'esprit et aux muscles des hommes et des femmes. Le travail des masses, leur volonté de vaincre les fléaux qui les ont des siècles durant exclues de l'histoire du cerveau humain doivent être branchés sur ceux de tous les Peuples sous-développés. Les nouvelles qui intéressent les peuples du tiers monde ne sont pas celles qui concernent le mariage du roi Baudouin ou les scandales de la bourgeoisie italienne. Ce que nous voulons savoir, ce sont les expériences faites par les Argentins ou les Birmans dans le cadre de la lutte contre l'analphabétisme ou les tendances dictatoriales des dirigeants. Ce sont des éléments qui nous renforcent, nous instruisent et décuplent notre efficacité. Comme on le voit, un programme est nécessaire à un gouvernement qui veut vraiment libérer politiquement et socialement le peuple. Programme économique mais aussi doctrine sur la répartition des richesses et sur les relations sociales. En fait, il faut avoir une conception de l'homme, une conception de l'avenir de l'humanité. Ce qui veut dire qu'aucune formule démagogique, aucune complicité avec l'ancien occupant ne remplace un programme. Les peuples, d'abord inconscients mais bientôt de plus en plus lucides, exigeront avec force ce programme. Les peuples africains, les peuples sous-développés, 
contrairement à ce que l'on a coutume de croire, édifient rapidement leur conscience politique et [193] sociale. Ce qui peut être grave, c'est que très souvent ils parviennent à cette conscience sociale avant la phase nationale. Aussi peut-on trouver dans les pays sous-développés l'exigence violente d'une justice sociale qui paradoxalement s'allie à un tribalisme très souvent primitif. Les peuples sous-développés ont un comportement de gens affamés. Ce qui signifie que les jours de ceux qui s'amusent en Afrique sont rigoureusement comptés. Nous voulons dire que leur pouvoir ne saurait se prolonger indéfiniment. Une bourgeoisie qui donne aux masses le seul aliment du nationalisme faillit à sa mission et s'empêtre nécessairement dans une succession de mésaventures. Le nationalisme, s'il n'est pas explicité, enrichi et approfondi, s'il ne se transforme pas très rapidement en conscience politique et sociale, en humanisme, conduit à une impasse. La direction bourgeoise des pays sous-développés confine la conscience nationale dans un formalisme stérilisant. Seul l'engagement massif des hommes et des femmes dans des tâches éclairées et fécondes donne contenu et densité à une conscience. Alors le drapeau et le palais de gouvernement cessent d'être les symboles de la nation. La nation déserte ces lieux illuminés et factices et se réfugie dans les campagnes où elle reçoit vie et dynamisme. L'expression vivante de la nation c'est la conscience en mouvement de l'ensemble du peuple. C'est la praxis cohérente et éclairée des hommes et des femmes. La construction collective d'un destin, c'est l'assomption d'une responsabilité à la dimension de l'histoire. Autrement, c'est l'anarchie, la répression, le surgissement des partis tribalisés, du fédéralisme, etc. Le gouvernement national, s'il veut être national, doit gouverner par le peuple et pour le peuple, pour les déshérités et par les déshérités. Aucun leader quelle que soit sa valeur ne peut se substituer à la volonté populaire et le gouvernement national doit, avant de se préoccuper de prestige international, redonner dignité à chaque citoyen, meubler les cerveaux, emplir les yeux de choses humaines, développer un panorama humain parce qu'habité par des hommes conscients et souverains. 
[195]

\section{LES DAMNÉS DE LA TERRE}

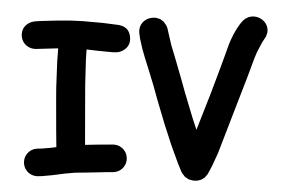

Retour à la table des matières 
[197]

\section{LES DAMNÉS DE LA TERRE}

\section{IV \\ Sur la culture nationale}

"Il ne suffit pas d'écrire un chant révolutionnaire pour participer à la révolution africaine, il faut faire cette révolution avec le peuple. Avec le peuple et les chants viendront seuls et d'eux-mêmes.

Pour avoir une action authentique, il faut être soimême une part vive de l'Afrique et de sa pensée, un élément de cette énergie populaire tout entière mobilisée pour la libération, le progrès et le bonheur de l'Afrique. II n'y a aucune place en dehors de ce seul combat ni pour l'artiste, ni pour l'intellectuel qui n'est pas lui-même engagé et totalement mobilisé avec le peuple dans le grand combat de l'A frique et de l'humanité souffrante. »

Sékou Touré 15.

Retour à la table des matières

15 «Le leader politique considéré comme le représentant d'une culture », communication au deuxième Congrès des écrivains et artistes noirs, Rome, 1959. 
Chaque génération doit dans une relative opacité découvrir sa mission, la remplir ou la trahir. Dans les pays sous-développés les générations précédentes ont à la fois résisté au travail d'érosion poursuivi par le colonialisme et préparé le mûrissement de luttes actuelles. Il nous faut perdre l'habitude, maintenant que nous sommes au cœur du combat, de minimiser l'action de nos pères ou de feindre l'incompréhension devant leur silence ou leur passivité. Ils se sont battus comme ils pouvaient, avec les armes qu'ils possédaient alors et si les échos de leur lutte n'ont pas retenti sur l'arène internationale. Il faut en [198] voir la raison moins dans l'absence d'héroïsme que dans une situation internationale fondamentalement différente. Il a fallu que plus d'un colonisé dise "ça ne peut plus durer», il a fallu que plus d'une tribu se rebelle, il a fallu plus d'une jacquerie matée, plus d'une manifestation réprimée pour que nous puissions aujourd'hui tenir tête avec cette certitude dans la victoire.

Notre mission historique, à nous qui avons pris la décision de briser les reins du colonialisme, est d'ordonner toutes les révoltes, tous les actes désespérés, toutes les tentatives avortées ou noyées dans le sang.

Nous analyserons dans ce chapitre le problème, que l'on sent fondamental, de la légitimité de la revendication d'une nation. Il faut reconnaître que le parti politique qui mobilise le peuple ne se préoccupe guère de ce problème de la légitimité. Les partis politiques partent du réel vécu et c'est au nom de ce réel, au nom de cette actualité qui pèse sur le présent et sur l'avenir des hommes et des femmes, qu'ils convient à l'action. Le parti politique peut bien parler en termes émouvants de la nation mais ce qui l'intéresse, c'est que le peuple qui l'écoute comprenne la nécessité de participer au combat s'il aspire tout simplement à exister. 
On sait aujourd'hui que, dans la première phase de la lutte nationale, le colonialisme essaie de désamorcer la revendication nationale en faisant de l'économisme. Dès les premières revendications le colonialisme feint la compréhension en reconnaissant avec une humilité ostentatoire que le territoire souffre d'un sous-développement grave qui nécessite un effort économique et social important.

Et, de fait, il arrive que certaines mesures spectaculaires, des chantiers de chômage ouverts çà et là, retardent de quelques années la cristallisation de la conscience nationale. Mais tôt ou tard, le colonialisme s'aperçoit qu'il ne lui est pas possible de [199] réaliser un projet de réformes économico-sociales qui satisfasse les aspirations des masses colonisées. Même sur le plan du ventre, le colonialisme fait la preuve de son impuissance congénitale. I'Etat colonialiste découvre très rapidement que vouloir désarmer les partis nationaux dans le domaine strictement économique reviendrait à faire aux colonies ce qu'il n'a pas voulu faire sur son propre territoire. Et ce n'est pas un hasard si fleurit aujourd'hui un peu partout la doctrine du cartiérisme.

L'amertume désabusée de Cartier face à l'obstination de la France à s'attacher des gens qu'elle devra nourrir alors que tant de Français vivent dans la gêne traduit l'impossibilité dans laquelle se trouve le colonialisme à se transformer en programme désintéressé d'aide et de soutien. C'est pourquoi encore une fois il ne faut pas perdre son temps à répéter qu'il vaut mieux la faim dans la dignité que le pain dans la servitude. Il faut au contraire se convaincre que le colonialisme est incapable de procurer aux peuples colonisés les conditions matérielles susceptibles de lui faire oublier son souci de dignité. Une fois que le colonialisme a compris où l'entraînerait sa tactique de réformes sociales, on le voit retrouver ses vieux réflexes, renforcer les effectifs de police, dépêcher des troupes et installer un régime de terreur mieux adapté à ses intérêts et à sa psychologie. 
Au sein des partis politiques, le plus souvent latéralement à eux, apparaissent des hommes de culture colonisés. Pour ces hommes la revendication d'une culture nationale, l'affirmation de l'existence de cette culture représente un champ de bataille privilégié. Alors que les hommes politiques inscrivent leur action dans le réel, les hommes de culture se situent dans le cadre de l'histoire. Face à l'intellectuel colonisé qui décide de répondre agressivement à la théorie colonialiste d'une barbarie antécoloniale, le colonialisme va peu réagir. Il réagira d'autant moins que les idées développées par la jeune intelligentzia colonisée sont largement professées par les spécialistes métropolitains. Il est banal, en effet, de constater que depuis plusieurs [200] décades de nombreux chercheurs européens ont, en gros, réhabilité les civilisations africaines, mexicaines ou péruviennes. On a pu s'étonner de la passion investie par les intellectuels colonisés pour défendre l'existence d'une culture nationale. Mais ceux qui condamnent cette passion exacerbée oublient singulièrement que leur psychisme, leur moi s'abritent commodément derrière une culture française ou allemande qui a fait ses preuves et que personne ne conteste.

Je concède que sur le plan de l'existence le fait qu'il ait existé une civilisation aztèque ne change pas grand-chose au régime alimentaire du paysan mexicain d'aujourd'hui. Je concède que toutes les preuves qui pourraient être données de l'existence d'une prodigieuse civilisation songhaï ne changent pas le fait que les Songhaïs d'aujourd'hui sont sous-alimentés, analphabètes, jetés entre ciel et eau, la tête vide, les yeux vides. Mais, on l'a dit à plusieurs reprises, cette recherche passionnée d'une culture nationale en deçà de l'ère coloniale tire sa légitimité du souci que partagent les intellectuels colonisés de prendre du recul par rapport à la culture occidentale dans laquelle ils risquent de s'enliser. Parce qu'ils se rendent compte qu'ils sont en train de se perdre, donc d'être perdus pour leur peuple, ces hommes, la rage au cœur et le cerveau fou, s'acharnent à reprendre contact avec la sève la plus ancienne, la plus antécoloniale de leur peuple. 
Allons plus loin, peut-être que ces passions et que cette rage sont entretenues ou du moins orientées par le secret espoir de découvrir au-delà de cette misère actuelle, de ce mépris pour soi-même, de cette démission et de ce reniement, une ère très belle et très resplendissante qui nous réhabilite, à la fois vis-à-vis de nous-mêmes et vis-à-vis des autres. Je dis que je suis décidé à aller loin. Inconsciemment peutêtre les intellectuels colonisés, ne pouvant faire l'amour avec l'histoire présente de leur peuple opprimé, ne pouvant s'émerveiller de l'histoire de leurs barbaries actuelles, ont-ils décidé d'aller plus loin, de descendre plus bas et c'est, n'en doutons point, dans une allégresse [201] exceptionnelle qu'ils ont découvert que le passé n'était point de honte mais de dignité, de gloire et de solennité. La revendication d'une culture nationale passée ne réhabilite pas seulement, ne fait pas que justifier une culture nationale future. Sur le plan de l'équilibre psychoaffectif elle provoque chez le colonisé une mutation d'une importance fondamentale. On n'a peut-être pas suffisamment montré que le colonialisme ne se contente pas d'imposer sa loi au présent et à l'avenir du pays dominé. Le colonialisme ne se satisfait pas d'enserrer le peuple dans ses mailles, de vider le cerveau colonisé de toute forme et de tout contenu. Par une sorte de perversion de la logique, il s'oriente vers le passé du peuple opprimé, le distord, le défigure, l'anéantit. Cette entreprise de dévalorisation de l'histoire d'avant la colonisation prend aujourd'hui sa signification dialectique.

Quand on réfléchit aux efforts qui ont été déployés pour réaliser l'aliénation culturelle si caractéristique de l'époque coloniale, on comprend que rien n'a été fait au hasard et que le résultat global recherché par la domination coloniale était bien de convaincre les indigènes que le colonialisme devait les arracher à la nuit. Le résultat, consciemment poursuivi par le colonialisme, était d'enfoncer dans la tête des indigènes que le départ du colon signifierait pour eux retour à la barbarie, encanaillement, animalisation. Sur le plan de l'inconscient, le colonialisme ne cherchait donc pas à être perçu par l'indigène comme une mère douce et bienveillante qui protège l'enfant d'un environnement 
hostile, mais bien sous la forme d'une mère qui, sans cesse, empêche un enfant fondamentalement pervers de réussir son suicide, de donner libre cours à ses instincts maléfiques. La mère coloniale défend l'enfant contre lui-même, contre son moi, contre sa physiologie, sa biologie, son malheur ontologique.

Dans cette situation la revendication de l'intellectuel colonisé n'est pas un luxe mais exigence de programme cohérent. L'intellectuel colonisé qui situe son combat sur le plan de la [202] légitimité, qui veut apporter des preuves, qui accepte de se mettre nu pour mieux exhiber l'histoire de son corps est condamné à cette plongée dans les entrailles de son peuple.

Cette plongée n'est pas spécifiquement nationale. L'intellectuel colonisé qui décide de livrer combat aux mensonges colonialistes, le livrera à l'échelle du continent. Le passé est valorisé. La culture, qui est arrachée du passé pour être déployée dans toute sa splendeur, n'est pas celle de son pays. Le colonialisme, qui n'a pas nuancé ses efforts, n'a cessé d'affirmer que le nègre est un sauvage et le nègre pour lui n'était ni l'Angolais, ni le Nigérien. Il parlait du nègre. Pour le colonialisme, ce vaste continent était un repaire de sauvages, un pays infesté de superstitions et de fanatisme, voué au mépris, lourd de la malédiction de Dieu, pays d'anthropophages, pays de nègres. La condamnation du colonialisme est continentale. L'affirmation par le colonialisme que la nuit humaine a caractérisé la période antécoloniale concerne l'ensemble du continent africain. Les efforts du colonisé pour se réhabiliter et échapper à la morsure coloniale s'inscrivent logiquement dans la même perspective que celle du colonialisme. L'intellectuel colonisé qui est parti très loin du côté de la culture occidentale et qui se met en tête de proclamer l'existence d'une culture ne le fait jamais au nom de l'Angola ou du Dahomey. La culture qui est affirmée est la culture africaine. Le nègre, qui n'a jamais été aussi nègre que depuis qu'il est dominé par le Blanc, quand il décide de faire preuve de culture, de faire œuvre de culture, s'aperçoit que l'histoire lui impose un terrain pré- 
cis, que l'histoire lui indique une voie précise et qu'il lui faut manifester une culture nègre.

Et il est bien vrai que les grands responsables de cette racialisation de la pensée, ou du moins des démarches de la pensée, sont et demeurent les Européens qui n'ont pas cessé d'opposer la culture blanche aux autres incultures. Le colonialisme n'a pas cru devoir perdre son temps à nier les unes après les autres les cultures des différentes nations. Aussi la réponse du colonisé [203] sera-t-elle d'emblée continentale. En Afrique, la littérature colonisée des vingt dernières années n'est pas une littérature nationale mais une littérature de nègres. Le concept de négritude par exemple était l'antithèse affective sinon logique de cette insulte que l'homme banc faisait à l'humanité. Cette négritude ruée contre le mépris du Blanc s'est révélée dans certains secteurs seule capable de lever interdictions et malédictions. Parce que les intellectuels guinéens ou kenyans se trouvaient confrontés avant tout à l'ostracisme global, au mépris syncrétique du dominateur, leur réaction fut de s'admirer et de se chanter. À l'affirmation inconditionnelle de la culture européenne a succédé l'affirmation inconditionnelle de la culture africaine. Dans l'ensemble les chantres de la négritude opposeront la vieille Europe à la jeune Afrique, la raison ennuyeuse à la poésie, la logique oppressive à la piaffante nature, d'un côté raideur, cérémonie, protocole, scepticisme, de l'autre ingénuité, pétulance, liberté, pourquoi pas luxuriance. Mais aussi irresponsabilité.

Les chantres de la négritude n'hésiteront pas à transcender les limites du continent. D'Amérique des voix noires vont reprendre cet hymne avec une ampleur accrue. Le «monde noir » verra le jour et Busia du Ghana, Birago Diop du Sénégal, Hampaté Ba du Soudan, SaintClair Drake de Chicago n'hésiteront pas à affirmer l'existence de liens communs, de lignes de force identiques.

L'exemple du monde arabe pourrait être également proposé ici. On sait que la majorité des territoires arabes a été soumise à la domina- 
tion coloniale. Le colonialisme a déployé dans ces régions les mêmes efforts pour ancrer dans l'esprit des indigènes que leur histoire d'avant la colonisation était une histoire dominée par la barbarie. La lutte de libération nationale s'est accompagnée d'un phénomène culturel connu sous le nom de réveil de l'Islam. La passion mise par les auteurs arabes contemporains à rappeler à leur peuple les grandes pages de l'histoire arabe est une réponse aux mensonges de l'occupant. Les grands noms de la littérature arabe ont été répertoriés et le passé de la [204] civilisation arabe a été brandi avec la même fougue, la même ardeur que celui des civilisations africaines. Les leaders arabes ont tenté de relancer cette fameuse Dar El Salam qui a rayonné si brillamment auX XII ${ }^{e}, \mathrm{XIII}^{e}$ et $\mathrm{XIV}{ }^{e}$ siècles.

Aujourd'hui, sur le plan politique, la Ligue arabe concrétise cette volonté de reprendre l'héritage du passé et de le faire culminer. Aujourd'hui, des médecins et des poètes arabes s'interpellent à travers les frontières, s'efforçant de lancer une nouvelle culture arabe, une nouvelle civilisation arabe. C'est au nom de l'arabisme que ces hommes se réunissent, c'est en son nom qu'ils s'efforcent de penser. Toutefois, dans le monde arabe, le sentiment national a conservé, même sous la domination coloniale, une vivacité que l'on ne retrouve pas en Afrique. Aussi ne décèle-t-on pas dans la Ligue arabe cette communion spontanée de chacun avec tous. Au contraire, paradoxalement, chacun essaie de chanter les réalisations de sa nation. Le phénomène culturel s'étant dégagé de l'indifférenciation qui le caractérisait dans le monde africain, les Arabes ne parviennent pas toujours à s'effacer devant l'objet. Le vécu culturel n'est pas national mais arabe. Le problème n'est pas encore d'assurer une culture nationale, pas encore de saisir le mouvement des nations, mais d'assumer une culture arabe ou africaine face à la condamnation globale portée par le dominateur. Sur le plan africain, comme sur le plan arabe, on voit que la revendication de l'homme de culture du pays colonisé est syncrétique, continentale, mondiale dans le cas des Arabes. 
Cette obligation historique dans laquelle se sont trouvés les hommes de culture africains de racialiser leurs revendications, de parler davantage de culture africaine que de culture nationale va les conduire à un cul-de-sac. Prenons par exemple le cas de la Société africaine de culture. Cette société a été créée par des intellectuels africains qui désiraient se connaître, échanger leurs expériences et leurs recherches respectives. Le but de cette société était donc d'affirmer l'existence d'une culture africaine, [205] d'inventorier cette culture dans le cadre de nations définies, de révéler le dynamisme interne de chacune des cultures nationales. Mais, dans le même temps, cette société répondait à une autre exigence: celle de se ranger aux côtés de la Société européenne de culture qui menaçait de se transformer en Société universelle de culture. Il y avait donc à la racine de cette décision le souci d'être présent au rendez-vous universel avec toutes ses armes, avec une culture jaillie des entrailles mêmes du continent africain. Or très rapidement cette Société va révéler son inaptitude à assumer ces différentes tâches et se limitera à des manifestations exhibitionnistes : montrer aux Européens qu'il existe une culture africaine, s'opposer aux Européens ostentatoires et narcissistes, tel sera le comportement habituel des membres de cette Société. Nous avons montré que cette attitude était normale et tirait sa légitimité du mensonge propagé par les hommes de culture occidentaux. Mais la dégradation des buts de cette Société va s'approfondir avec l'élaboration du concept de négritude. La Société africaine va devenir la société culturelle du monde noir et sera amenée à inclure la diaspora nègre, c'est-àdire les dizaines de millions de Noirs répartis sur les continents américains.

Les nègres qui se trouvent aux États-Unis, en Amérique centrale ou latine avaient en effet besoin de se raccrocher à une matrice culturelle. Le problème qui se posait à eux n'était pas fondamentalement différent de celui auquel se trouvaient confrontés les Africains. À leur égard les Blancs d'Amérique ne se sont pas comportés différemment de ceux qui dominaient les Africains. Nous avons vu que les Blancs s'étaient accoutumés à mettre tous les nègres dans le même sac. Au 
cours du premier congrès de la Société africaine de culture qui s'est tenu à Paris en 1956, les nègres américains ont spontanément pensé leurs problèmes sur le même plan que ceux de leurs congénères africains. Les hommes de culture africains en parlant de civilisations africaines décernaient un état civil raisonnable aux anciens esclaves. Mais, progressivement, les nègres américains se sont aperçus que les problèmes existentiels qui se posaient à eux ne recoupaient pas ceux auxquels étaient confrontés les nègres [206] africains. Les nègres de Chicago ne ressemblaient aux Nigériens et aux Tanganyikais que dans l'exacte mesure où ils se définissaient tous par rapport aux Blancs. Mais les premières confrontations passées, dès que la subjectivité s'est trouvée tranquillisée, les nègres américains se sont aperçus que les problèmes objectifs étaient fondamentalement hétérogènes. Les cars de la liberté où Noirs et Blancs américains tentent de faire reculer la discrimination raciale n'entretiennent dans leur principe et leurs objectifs que peu de rapports avec la lutte héroïque du peuple angolais contre l'odieux colonialisme portugais. Aussi au cours du deuxième congrès de la Société africaine de culture les nègres américains décidaient-ils la création d'une Société américaine des hommes de culture noirs.

La négritude trouvait donc sa première limite dans les phénomènes qui rendent compte de l'historicisation des hommes. La culture nègre, la culture négro-africaine se morcelait parce que les hommes qui se proposaient de l'incarner se rendaient compte que toute culture est d'abord nationale et que les problèmes qui maintenaient Richard Wright ou Langston Hughes en éveil étaient fondamentalement différents de ceux que pouvaient affronter Léopold Senghor ou Jomo Kenyatta. De même certains États arabes qui avaient pourtant entonné le chant prestigieux de la rénovation arabe devaient-ils s'apercevoir que leur position géographique, l'interdépendance économique de leur région étaient plus fortes que le passé que l'on voulait revivre. Aussi trouvons-nous aujourd'hui les États arabes organiquement rattachés aux sociétés méditerranéennes de culture. C'est que ces États sont 
soumis à des pressions modernes, à des nouveaux circuits commerciaux tandis que les réseaux qui dominaient au cours du rayonnement arabe ont disparu. Mais il y a surtout le fait que les régimes politiques de certains États arabes sont à ce point hétérogènes, étrangers les uns aux autres, qu'une rencontre même culturelle entre ces États se révèle un non-sens.

On voit donc que le problème culturel, tel qu'il est quelquefois posé dans les pays colonisés, risque de donner lieu à des [207] ambiguités graves. L'inculture des nègres, que proclame le colonialisme, la barbarie congénitale des Arabes devaient logiquement conduire à une exaltation des phénomènes culturels non plus nationaux mais continentaux et singulièrement racialisés. En Afrique, la démarche de l'homme de culture est une démarche négro-africaine ou arabo-musulmane. Elle n'est pas spécifiquement nationale. La culture est de plus en plus coupée de l'actualité. Elle trouve refuge dans un foyer passionnellement incandescent et se fraie difficilement des voies concrètes qui seraient pourtant les seules susceptibles de lui procurer les attributs de fécondité, d'homogénéité et de densité.

Si l'entreprise de l'intellectuel colonisé est historiquement limitée, il reste qu'elle contribue dans une large mesure à soutenir, à légitimer l'action des hommes politiques. Et il est vrai que la démarche de l'intellectuel colonisé prend quelquefois les aspects d'un culte, d'une religion. Mais si l'on veut bien analyser comme, il faut cette attitude, on s'aperçoit qu'elle traduit la prise de conscience par le colonisé du danger qu'il court de rompre les dernières amarres avec son peuple. Cette foi proclamée en l'existence d'une culture nationale est en fait un retour ardent, désespéré vers n'importe quoi. Pour assurer son salut, pour échapper à la suprématie de la culture blanche, le colonisé sent la nécessité de revenir vers des racines ignorées, de se perdre, advienne que pourra, dans ce peuple barbare. Parce qu'il se sent devenir aliéné, c'est-à-dire le lieu vivant de contradictions qui menacent d'être insurmontables, le colonisé s'arrache du marais où il risquait de s'enliser et à corps perdu, à cerveau perdu il accepte, il décide 
d'assumer, il confirme. Le colonisé se découvre tenu de répondre de tout et de tous. Il ne se fait pas seulement le défenseur, il accepte d'être mis avec les autres et dorénavant il peut se permettre de rire de sa lâcheté passée.

Cet arrachement pénible et douloureux est cependant nécessaire. Faute de le réaliser on assistera à des mutilations psychoaffectives extrêmement graves. Des gens sans rivage, sans [208] limite, sans couleur, des apatrides, des non-enracinés, des anges. Également on ne sera pas étonné d'entendre certains colonisés déclarer: "C'est en tant que Sénégalais et Français... C'est en tant qu'Algérien et Français... que je parle. » Butant sur la nécessité, s'il veut être véridique, d'assumer deux nationalités, deux déterminations, l'intellectuel arabe et français, l'intellectuel nigérien et anglais choisit la négation de l'une de ces déterminations. Le plus souvent, ne voulant ou ne pouvant pas choisir, ces intellectuels ramassent toutes les déterminations historiques qui les ont conditionnés et se placent radicalement dans une «perspective universelle».

C'est que l'intellectuel colonisé s'est jeté avec avidité dans la culture occidentale. Semblable aux enfants adoptifs, qui ne cessent leurs investigations du nouveau cadre familial que dans le moment où se cristallise dans leur psychisme un noyau sécurisant minimum, l'intellectuel colonisé va tenter de faire sienne la culture européenne. Il ne se contentera pas de connaître Rabelais ou Diderot, Shakespeare ou Edgar Poe, il bandera son cerveau jusqu'à la plus extrême complicité avec ces hommes.

« La dame n'était pas seule

Elle avait un mari

Un mari très comme il faut

Qui citait Racine et Corneille

Et Voltaire et Rousseau 


\section{Et le Père Hugo et le jeune Musset \\ Et Gide et Valéry \\ Et tant d'autres encore ${ }^{16}$. »}

Mais au moment où les partis nationalistes mobilisent le peuple au nom de l'indépendance nationale, l'intellectuel colonisé peut quelquefois rejeter du pied ces acquisitions qu'il [209] ressent soudain comme aliénantes. Toutefois il est plus facile de proclamer qu'on rejette que de rejeter réellement. Cet intellectuel qui, par le truchement de la culture, s'était infiltré dans la civilisation occidentale, qui était arrivé à faire corps, c'est-à-dire à changer de corps, avec la civilisation européenne va s'apercevoir que la matrice culturelle, qu'il voudrait assumer par souci d'originalité, ne lui offre guère les figures de proue capables de supporter la comparaison avec celles, nombreuses et prestigieuses, de la civilisation de l'occupant. L'histoire, bien sûr, écrite d'ailleurs par des occidentaux et à l'intention des occidentaux, pourra épisodiquement valoriser certaines périodes du passé africain. Mais, debout devant le présent de son pays, observant lucidement «objectivement » l'actualité du continent qu'il voudrait faire sien, l'intellectuel est effrayé par le vide, l'abrutissement, la sauvagerie. Or il sent qu'il lui faut sortir de cette culture blanche, qu'il lui faut chercher ailleurs, n'importe où, et faute de trouver un aliment culturel à la mesure du panorama glorieux étalé par le dominateur, l'intellectuel colonisé très souvent va refluer sur des positions passionnelles et développera une psychologie dominée par une sensibilité, une sensitivité, une susceptibilité exceptionnelles. Ce mouvement de repli qui procède d'abord d'une pétition de principe dans son mécanisme interne et sa physionomie évoque surtout un réflexe, une contraction musculaire.

Ainsi s'explique suffisamment le style des intellectuels colonisés qui décident d'exprimer cette phase de la conscience en train de se

16 René Depestre, Face à la nuit. 
libérer. Style heurté, fortement imagé car l'image est le pont-levis qui permet aux énergies inconscientes de s'éparpiller dans les prairies environnantes. Style nerveux, animé de rythmes, de part en part habité par une vie éruptive. Coloré aussi, bronzé, ensoleillé et violent. Ce style, qui a en son temps étonné les occidentaux, n'est point comme on a bien voulu le dire un caractère racial mais traduit avant tout un corps à corps, révèle la nécessité dans laquelle s'est trouvé cet homme de se faire mal, de saigner réellement de sang rouge, de se libérer [210] d'une partie de son être qui déjà renfermait des germes de pourriture. Combat douloureux, rapide, où immanquablement le muscle devait se substituer au concept.

Si sur le plan poétique cette démarche atteint des hauteurs inaccoutumées, il demeure que sur le plan de l'existence l'intellectuel débouche fréquemment sur une impasse. Lorsque, parvenu à l'apogée du rut avec son peuple quel qu'il fût et quel qu'il soit, l'intellectuel décide de retrouver le chemin de la quotidienneté, il ne ramène de son aventure que des formules terriblement infécondes. Il privilégie les coutumes, les traditions, les modes d'apparaître et sa quête forcée, douloureuse ne fait qu'évoquer une banale recherche d'exotisme. C'est la période où les intellectuels chantent les moindres déterminations du panorama indigène. Le boubou se trouve sacralisé, les chaussures parisiennes ou italiennes délaissées au profit des babouches. Le langage du dominateur écorche soudain les lèvres. Retrouver son peuple c'est quelquefois dans cette période vouloir être nègre, non un nègre pas comme les autres mais un véritable nègre, un chien de nègre, tel que le veut le Blanc. Retrouver son peuple c'est se faire bicot, se faire le plus indigène possible, le plus méconnaissable, c'est se couper les ailes qu'on avait laissé pousser.

L'intellectuel colonisé décide de procéder à l'inventaire des mauvaises manières puisées dans le monde colonial et se dépêche de se rappeler les bonnes manières du peuple, de ce peuple dont on a décidé qu'il 
détenait toute vérité. Le scandale que déclenche cette démarche dans les rangs des colonialistes installés sur le territoire renforce la décision du colonisé. Lorsque les colonialistes, qui avaient savouré leur victoire sur ces assimilés, se rendent compte que ces hommes que l'on croyait sauvés commencent à se dissoudre dans la négraille, tout le système vacille. Chaque colonisé gagné, chaque colonisé qui était passé aux aveux, lorsqu'il décide de se perdre est non seulement un échec pour l'entreprise coloniale, mais symbolise encore l'inutilité et le manque de profondeur du travail accompli. [211] Chaque colonisé qui repasse la ligne est une condamnation radicale de la méthode et du régime et l'intellectuel colonisé trouve dans le scandale qu'il provoque une justification à sa démission et un encouragement à persévérer.

Si nous voulions retrouver à travers les œuvres d'écrivains colonisés les différentes phases qui caractérisent cette évolution, nous verrions se profiler devant nos yeux un panorama en trois temps. Dans une première phase, l'intellectuel colonisé prouve qu'il a assimilé la culture de l'occupant. Ses œuvres correspondent point par point à celles de ses homologues métropolitains. L'inspiration est européenne et on peut aisément rattacher ces œuvres à un courant bien défini de la littérature métropolitaine. C'est la période assimilationniste intégrale. On trouvera dans cette littérature de colonisé des parnassiens, des symbolistes, des surréalistes.

Dans un deuxième temps le colonisé est ébranlé et décide de se souvenir. Cette période de création correspond approximativement à la replongée que nous venons de décrire. Mais comme le colonisé n'est pas inséré dans son peuple, comme il entretient des relations d'extériorité avec son peuple, il se contente de se souvenir. De vieux épisodes d'enfance seront ramenés du fond de sa mémoire, de vieilles légendes seront réinterprétées en fonction d'une esthétique d'emprunt et d'une conception du monde découverte sous d'autres cieux. Quelquefois cette littérature de pré-combat sera dominée par l'humour et par l'allégorie. Période d'angoisse, de malaise, expérience de la mort, expé- 
rience aussi de la nausée. On se vomit, mais déjà, par en dessous, s'amorce le rire.

Enfin dans une troisième période, dite de combat, le colonisé, après avoir tenté de se perdre dans le peuple, de se perdre avec le peuple, va au contraire secouer le peuple. Au lieu de privilégier la léthargie du peuple il se transforme en réveilleur de peuple. Littérature de combat, littérature révolutionnaire, littérature nationale. Au cours de cette phase un grand nombre d'hommes et de femmes qui auparavant n'auraient jamais songé [212] à faire œuvre littéraire, maintenant qu'ils se trouvent placés dans des situations exceptionnelles, en prison, au maquis ou à la veille de leur exécution ressentent la nécessité de dire leur nation, de composer la phrase qui exprime le peuple, de se faire le porte-parole d'une nouvelle réalité en actes.

L'intellectuel colonisé cependant tôt ou tard se rendra compte qu'on ne prouve pas sa nation à partir de la culture mais qu'on la manifeste dans le combat que mène le peuple contre les forces d'occupation. Aucun colonialisme ne tire sa légitimité de l'inexistence culturelle des territoires qu'il domine. On ne fera jamais honte au colonialisme en déployant devant son regard des trésors culturels méconnus. L'intellectuel colonisé dans le moment même où il s'inquiète de faire œuvre culturelle ne se rend pas compte qu'il utilise des techniques et une langue empruntées à l'occupant. Il se contente de revêtir ces instruments d'un cachet qui se veut national mais qui rappelle étrangement l'exotisme. L'intellectuel colonisé qui revient à son peuple à travers les œuvres culturelles se comporte en fait comme un étranger. Quelquefois il n'hésitera pas à utiliser les dialectes pour manifester sa volonté d'être le plus près possible du peuple mais les idées qu'il exprime, les préoccupations qui l'habitent sont sans commune mesure avec la situation concrète que connaissent les hommes et les femmes de son pays. La culture vers laquelle se penche l'intellectuel n'est très souvent qu'un stock de particularismes. Voulant coller au peuple, il colle au revêtement visible. Or ce revêtement n'est qu'un reflet d'une vie souterraine, dense, en perpétuel renouvellement. Cette objectivité qui 
crève les yeux et qui semble caractériser le peuple n'est en fait que le résultat inerte et déjà nié d'adaptations multiples et pas toujours cohérentes d'une substance plus fondamentale qui, elle, est en plein renouvellement. L'homme de culture au lieu de partir à la recherche de cette substance va se laisser hypnotiser par ces lambeaux momifiés qui, stabilisés, signifient au contraire la négation, le dépassement, l'invention. La culture n'a jamais la translucidité de la coutume. La culture [213] fuit éminemment toute simplification. Dans son essence elle est à l'opposé de la coutume qui, elle, est toujours une détérioration de la culture. Vouloir coller à la tradition ou réactualiser les traditions délaissées, c'est non seulement aller contre l'histoire mais contre son peuple. Quand un peuple soutient une lutte année ou même politique contre un colonialisme implacable, la tradition change de signification. Ce qui était technique de résistance passive peut, dans cette période, être radicalement condamné. Dans un pays sous-développé en phase de lutte les traditions sont fondamentalement instables et sillonnées de courants centrifuges. C'est pourquoi l'intellectuel risque souvent d'être à contretemps. Les peuples qui ont mené la lutte sont de plus en plus imperméables à la démagogie et à vouloir trop les suivre on se révèle n'être qu'un vulgaire opportuniste, voire un retardataire.

Sur le plan des arts plastiques par exemple, le créateur colonisé qui coûte que coûte veut faire cuvre nationale se confine dans une reproduction stéréotypée des détails. Ces artistes, qui ont cependant approfondi les techniques modernes et participé aux grands courants de la peinture ou de l'architecture contemporaine, tournent le dos, contestent la culture étrangère et partant à la recherche du vrai national privilégient ce qu'ils croient être les constantes d'un art national. Mais ces créateurs oublient que les formes de pensée, que l'alimentation, les techniques modernes d'information, du langage et de l'habillement ont réorganisé dialectiquement le cerveau du peuple et que les constantes qui furent les garde-fous pendant la période coloniale sont en train de subir des mutations terriblement radicales. 
Ce créateur qui décide de décrire la vérité nationale se tourne paradoxalement vers le passé, vers l'inactuel. Ce qu'il vise dans son intentionnalité profonde, ce sont les déjections de la pensée, le dehors, les cadavres, le savoir définitivement stabilisé. Or l'intellectuel colonisé qui veut faire cuvre authentique doit savoir que la vérité nationale c'est d'abord la réalité nationale. Il lui faut pousser jusqu'au lieu en ébullition où se préfigure le savoir.

[214]

Avant l'indépendance le peintre colonisé était insensible au panorama national. Il privilégiait donc le non-figuratif ou, plus souvent, se spécialisait dans les natures mortes. Après l'indépendance son souci de rejoindre le peuple le confinera dans la représentation point par point de la réalité nationale. Il s'agit là d'une représentation non rythmée, sereine, immobile, qui évoque non pas la vie, mais la mort. Les milieux éclairés s'extasient devant cette vérité bien rendue mais on a le droit de se demander si cette vérité est réelle, si en fait elle n'est pas dépassée, niée, remise en question par l'épopée à travers laquelle le peuple se fraie un chemin vers l'histoire.

Sur le plan de la poésie nous pourrions faire les mêmes constatations. Après la phase assimilationniste de la poésie rimée, éclate le rythme du tam-tam poétique. Poésie de révolte, mais poésie analytique, descriptive. Le poète doit cependant comprendre que rien ne remplace l'engagement rationnel et irréversible aux côtés du peuple en armes. Encore une fois citons Depestre 17 :

17 René Depestre, Face à la nuit. 
« La dame n'était pas seule

Elle avait un mari

Un mari qui savait tout

Mais à parler franc qui ne savait rien

Parce que la culture ne va pas sans concessions

Une concession de sa chair et de son sang

Une concession de soi-même aux autres

Une concession qui vaut le

Classicisme et le romantisme

Et tout ce dont on abreuve notre esprit. »

Le poète colonisé qui se préoccupe de faire œuvre nationale, qui s'obstine à décrire son peuple rate son but car il ne se met pas avant de dire en état de faire cette concession fondamentale [215] dont parle Depestre. Le poète français René Char l'a bien compris qui rappelle que «le poème émerge d'une imposition subjective et d'un choix objectif. Le poème est une assemblée en mouvement de valeurs originales déterminantes, en relations contemporaines avec quelqu'un que cette circonstance fait premier $18 \gg$.

Oui, le premier devoir du poète colonisé est de déterminer clairement le sujet peuple de sa création. On ne peut avancer résolument que si l'on prend d'abord conscience de son aliénation. Nous avons tout pris de l'autre côté. Or l'autre côté ne nous donne rien sans, par mille détours, nous courber dans sa direction, sans, par dix mille artifices, cent mille ruses, nous attirer, nous séduire, nous emprisonner. Prendre, c'est également, sur de multiples plans, être pris. Il ne suffit donc pas d'essayer de se déprendre en accumulant les proclamations ou les dénégations. Il ne suffit pas de rejoindre le peuple dans ce passé où il n'est plus mais dans ce mouvement basculé qu'il vient d'ébaucher et à partir duquel subitement tout va être mis en question. C'est dans ce

18 René Char, Partage formel. 
lieu de déséquilibre occulte où se tient le peuple qu'il faut que nous nous portions car, n'en doutons point, c'est là que se givre son âme et que s'illuminent sa perception et sa respiration.

Keita Fodeba, aujourd'hui ministre de l'Intérieur de la République de Guinée, lorsqu'il était directeur des Ballets africains n'a pas rusé avec la réalité que lui offrait le peuple de Guinée. Dans une perspective révolutionnaire, il a réinterprété toutes les images rythmiques de son pays. Mais il a fait davantage. Dans son cuvre poétique, peu connue, on trouve un constant souci de préciser le moment historique de la lutte, de délimiter le champ où se déroulera l'action, les idées autour desquelles se cristallisera la volonté populaire. Voici un poème de Keita Fodeba, authentique invitation à la réflexion, à la démystification, au combat.

[216]

\section{AUBE AFRICAINE}

(Musique de guitare)

C'était l'aube. Le petit hameau qui avait dansé toute la moitié de la nuit au son des tam-tams s'éveillait peu à peu. Les bergers en loques et jouant de la flûte conduisaient les troupeaux dans la vallée. Les jeunes filles, armées de canaris, se suivaient à la queue-leu-leu sur le sentier tortueux de la fontaine. Dans la cour du marabout, un groupe d'enfants chantonnait en chœur des versets du Koran.

\section{(Musique de guitare)}

C'était l'aube. Combat du jour et de la nuit. Mais celle-ci exténuée n'en pouvait plus, et, lentement expirait. Quelques 
rayons du soleil en signe avant-coureur de cette victoire du jour traînaient encore, timides et pâles, à l'horizon, les dernières étoiles doucement glissaient sous des tas de nuages, pareils aux flamboyants en fleurs.

\section{(Musique de guitare)}

C'était l'aube. Et là-bas au fond de la vaste plaine aux contours de pourpre, une silhouette d'homme courbé défrichait: silhouette de Naman, le cultivateur. À chaque coup de sa daba, les oiseaux effrayés s'envolaient et, à tire-d'aile, rejoignaient les rives paisibles du Djoliba, le grand fleuve Niger. Son pantalon de cotonnade grise, trempé de rosée, battait l'herbe sur les côtés. Il suait, infatigable, toujours courbé, maniant adroitement son outil; car il fallait que ses graines soient enfouies avant les prochaines pluies.

\section{(Musique du cora)}

C'était l'aube. Toujours l'aube. Les mange-mil, dans les feuillages, virevoltaient, annonçant le jour. Sur la piste humide [217] de la plaine, un enfant, portant en bandoulière son petit sac de flèches, courait essoufflé dans la direction de Naman. Il interpellait: «Frère Naman, le chef du hameau vous demande sous l'arbre à palabres. »

(Musique de cora)

Surpris d'une convocation aussi matinale, le cultivateur posa son outil et marcha vers le bourg qui maintenant radiait dans les lueurs du soleil naissant. Déjà, les Anciens, plus graves que ja- 
mais, siégeaient. À côté d'eux un homme en uniforme, un gardecercle, impassible, fumait tranquillement sa pipe.

(Musique de cora)

Naman prit place sur une peau de mouton. Le griot du chef se leva pour transmettre à l'assemblée la volonté des Anciens: "Les Blancs ont envoyé un garde-cercle pour demander un homme du hameau qui ira à la guerre dans leur pays. Les notables, après délibération, ont décidé de désigner le jeune homme le plus représentatif de notre race afin qu'il aille prouver à la bataille des Blancs le courage qui a toujours caractérisé notre Manding. »

\section{(Musique de guitare)}

Naman, dont chaque soir les jeunes filles en couplets harmonieux louaient l'imposante stature et le développement apparent des muscles, fut d'office désigné. La douce Kadia, sa jeune femme, bouleversée par la nouvelle, cessa soudain de piler, rangea le mortier sous le grenier et, sans mot dire, s'enferma dans sa case pour pleurer son malheur en sanglots étouffés. La mort lui ayant ravi son premier mari, elle ne pouvait concevoir que les Blancs lui enlèvent Naman, celui en qui reposaient tous ses nouveaux espoirs. 


\section{(Musique de guitare)}

\section{[218]}

Le lendemain, malgré ses larmes et ses plaintes, le son grave des tam-tams de guerre accompagna Naman au petit port du village où il s'embarqua sur un chaland à destination du chef-lieu de cercle. La nuit, au lieu de danser sur la place publique comme d'habitude, les jeunes filles vinrent veiller dans l'antichambre de Naman où elles contèrent jusqu'au matin autour d'un feu de bois.

\section{(Musique de guitare)}

Plusieurs mois s'écoulèrent sans qu'aucune nouvelle de Naman ne parvint au bourg. La petite Kadia s'en inquiéta si bien qu'elle eut recours à l'expert féticheur du village voisin. Les Anciens eux-mêmes tinrent sur le sujet un bref conciliabule secret dont rien ne transpira.

\section{(Musique de cora)}

Un jour enfin arriva au village une lettre de Naman à l'adresse de Kadia. Celle-ci, soucieuse de la situation de son époux, se rendit la même nuit, après de pénibles heures de marche, au chef-lieu de cercle où un traducteur lut la missive.

Naman était en Afrique du Nord, en bonne santé et il demandait des nouvelles de la moisson, des fêtes de la mare, des danses, de l'arbre à palabres, du village... 


\section{(Balafong)}

Cette nuit, les commères accordèrent à la jeune Kadia la faveur d'assister, dans la cour de leur doyenne, à leurs palabres coutumières des soirs. Le chef de village, heureux de la nouvelle, offrit un grand festin à tous les mendiants des environs.

\section{(Balafong)}

Plusieurs mois s'écoulèrent encore et tout le monde redevenait anxieux car on ne savait plus rien de Naman. Kadia envisageait [219] d'aller de nouveau consulter le féticheur lorsqu'elle reçut une deuxième lettre. Naman, après la Corse et l'Italie, était maintenant en Allemagne et il se félicitait d'être déjà décoré.

\section{(Balafong)}

Une autre fois c'était une simple carte qui apprenait que Naman était fait prisonnier des Allemands. Cette nouvelle pesa sur le village de tout son poids. Les Anciens tinrent conseil et décidèrent que Naman était désormais autorisé à danser le Douga, cette danse sacrée du vautour que nul ne danse sans avoir fait une action d'éclat, cette danse des empereurs malinkés dont chaque pas est une étape de l'histoire du Mali. Ce fut là une consolation pour Kadia de voir son mari élevé à la dignité des héros du pays. 


\section{(Musique de guitare)}

Le temps passa... Deux années se suivirent... Naman était toujours en Allemagne. Il n'écrivait plus.

\section{(Musique de guitare)}

Un beau jour, le chef du village reçut de Dakar quelques mots qui annonçaient l'arrivée prochaine de Naman.

Aussitôt, les tam-tams crépitèrent. On dansa et chanta jusqu'à l'aube. Les jeunes filles composèrent de nouveaux airs pour sa réception car les anciens qui lui étaient dédiés ne disaient rien du Douga, cette célèbre danse du Manding.

\section{(Tam-tams)}

Mais, un mois plus tard, caporal Moussa, un grand ami de $\mathrm{Na}-$ man, adressa cette tragique lettre à Kadia: "C'était l'aube. Nous étions à Tiaroye-sur-Mer. Au cours d'une grande querelle qui nous opposait à nos chefs blancs de Dakar, une balle a trahi Naman. Il repose en terre sénégalaise. » 
[220]

(Musique de guitare)

En effet, c'était l'aube. Les premiers rayons de soleil frôlant à peine la surface de la mer doraient ses petites vagues moutonnantes. Au souffle de la brise, les palmiers, comme écœurés par ce combat matinal, inclinaient doucement leurs troncs vers l'océan. Les corbeaux, en bandes bruyantes, venaient annoncer aux environs, par leur croassement, la tragédie qui ensanglantait l'aube de Tiaroye... Et, dans l'azur incendié, juste au-dessus du cadavre de Naman, un gigantesque vautour planait lourdement. Il semblait lui dire: "Naman! Tu n'as pas dansé cette danse qui porte mon nom. D'autres la danseront. »

\section{(Musique de cora)}

Si j'ai choisi ce long poème, c'est à cause de son incontestable valeur pédagogique. Ici, les choses sont claires. C'est un exposé précis, progressif. La compréhension du poème n'est pas seulement une démarche intellectuelle, mais une démarche politique. Comprendre ce poème c'est comprendre le rôle qu'on a à jouer, identifier sa démarche, fourbir ses armes. Il n'y a pas un colonisé qui ne reçoive le message contenu dans ce poème. Naman, héros des champs de bataille d'Europe, Naman qui ne cessa d'assurer à la métropole puissance et pérennité, Naman mitraillé par les forces de police au moment où il reprend contact avec sa terre natale, c'est Sétif en 1945, Fort-de-France, Saïgon, Dakar, Lagos. Tous ces nègres et tous ces bicots qui se sont battus pour défendre la liberté de la France ou la civilisation britannique se retrouvent dans ce poème de Keita Fodeba.

Mais Keita Fodeba voit plus loin. Dans les pays colonisés, le colonialisme, après avoir utilisé les autochtones sur les champs de bataille, 
les utilise comme anciens combattants pour briser les mouvements d'indépendance. Les associations d'anciens combattants sont aux colonies une des forces les plus antinationalistes qui soient. Le poète Keita Fodeba préparait le ministre [221] de l'Intérieur de la République de Guinée à déjouer les complots organisés par le colonialisme français. C'est, en effet, avec l'aide des anciens combattants que les services secrets français entendaient entre autres briser la jeune indépendance guinéenne.

L'homme colonisé qui écrit pour son peuple, quand il utilise le passé, doit le faire dans l'intention d'ouvrir l'avenir, d'inviter à l'action, de fonder l'espoir. Mais pour assurer l'espoir, pour lui donner densité, il faut participer à l'action, s'engager corps et âme dans le combat national. On peut parler de tout mais quand on décide de parler de cette chose unique dans la vie d'un homme que représente le fait d'ouvrir I'horizon, de porter la lumière chez soi, de mettre debout soi-même et son peuple, alors il faut musculairement collaborer.

La responsabilité de l'homme de culture colonisé n'est pas une responsabilité en face de la culture nationale mais une responsabilité globale à l'égard de la nation globale, dont la culture n'est, somme toute, qu'un aspect. L'homme de culture colonisé ne doit pas se préoccuper de choisir le niveau de son combat, le secteur où il décide de livrer le combat national, se battre pour la culture nationale, c'est d'abord se battre pour la libération de la nation, matrice matérielle à partir de laquelle la culture devient possible. Il n'y a pas un combat culturel qui se développerait latéralement au combat populaire. Par exemple, tous ces hommes et toutes ces femmes qui se battent poings nus contre le colonialisme français en Algérie ne sont pas étrangers à la culture nationale algérienne. La culture nationale algérienne prend corps et consistance au cours de ces combats, en prison, devant la guillotine, dans les postes militaires français investis et détruits.

Il ne faut donc pas se contenter de plonger dans le passé du peuple pour y trouver des éléments de cohérence vis-à-vis des entreprises 
falsificatrices et péjoratives du colonialisme. Il faut travailler, lutter à la même cadence que le peuple afin de préciser l'avenir, préparer le terrain où déjà se dressent des pousses vigoureuses. La culture nationale n'est pas le folklore où un populisme abstrait a cru découvrir la vérité du peuple. Elle n'est [222] pas une masse sédimentée de gestes purs, c'est-à-dire de moins en moins rattachables à la réalité présente du peuple. La culture nationale est l'ensemble des efforts faits par un peuple sur le plan de la pensée pour décrire, justifier et chanter l'action à travers laquelle le peuple s'est constitué et s'est maintenu. La culture nationale, dans les pays sous-développés, doit donc se situer au centre même de la lutte de libération que mènent ces pays. Les hommes de culture africains qui se battent encore au nom de la culture négro-africaine, qui ont multiplié les congrès au nom de l'unité de cette culture doivent aujourd'hui se rendre compte que leur activité s'est ramenée à confronter des pièces ou à comparer des sarcophages.

Il n'y a pas de communauté de destin des cultures nationales sénégalaise et guinéenne mais communauté de destin des nations guinéenne et sénégalaise dominées par le même colonialisme français. Si l'on veut que la culture nationale sénégalaise ressemble à la culture nationale guinéenne, il ne suffit pas que les dirigeants des deux peuples décident de poser les problèmes dans des perspectives voisines : problème de la libération, problèmes syndicaux, problèmes économiques. Même alors il ne saurait y avoir d'identité absolue car la cadence du peuple et celle des dirigeants ne sont pas uniformes.

Il ne saurait y avoir de cultures rigoureusement identiques. Imaginer qu'on fera de la culture noire, c'est oublier singulièrement que les nègres sont en train de disparaître, ceux qui les ont créés étant en train d'assister à la dissolution de leur suprématie économique et culturelle ${ }^{19}$. Il n'y aura pas de culture noire parce [223] qu'aucun

19 À la dernière distribution des prix à Dakar, le président de la République sénégalaise, Léopold Senghor, a décidé d'inscrire dans les programmes l'étude de la no- 
homme politique ne s'imagine avoir vocation de donner naissance à des républiques noires. Le problème est de savoir la place que ces hommes ont l'intention de réserver à leur peuple, le type de relations sociales qu'ils décident d'instaurer, la conception qu'ils se font de l'avenir de l'humanité. C'est cela qui compte. Tout le reste est littérature et mystification.

En 1959, les hommes de culture africains réunis à Rome n'ont cessé de parler de l'unité. Mais l'un des plus grands chantres de cette unité culturelle, Jacques Rabemananjara, est aujourd'hui ministre du gouvernement malgache et à ce titre a décidé avec son gouvernement de prendre position contre le peuple algérien à l'Assemblée générale des Nations unies. Rabe, s'il était fidèle à lui-même, aurait dû démissionner de ce gouvernement, dénoncer les hommes qui prétendent incarner la volonté du peuple malgache. Les quatre-vingt-dix mille morts de Madagascar n'ont pas donné mission à Rabe de s'opposer, à l'Assemblée générale des Nations unies, aux aspirations du peuple algérien.

La culture négro-africaine, c'est autour de la lutte des peuples qu'elle se densifie et non autour des chants, des poèmes ou du folklore: Senghor, qui est également membre de la Société africaine de culture et qui a travaillé avec nous autour de cette question de la culture africaine, n'a pas craint, lui non plus, de donner l'ordre à sa délégation d'appuyer les thèses françaises sur l'Algérie. L'adhésion à la culture négro-africaine, à l'unité culturelle de l'Afrique passe d'abord par un soutien inconditionnel à la lutte de libération des peuples. On ne peut vouloir le rayonnement de la culture africaine si l'on ne contribue pas concrètement à l'existence des conditions de cette culture, c'està-dire à la libération du continent.

tion de négritude. Si le souci exprimé par le président de la République du Sénégal est d'ordre historique, on ne peut qu'être d'accord. Si, au contraire, il s'agit de fabriquer des consciences noires, c'est tout simplement tourner le dos à I'histoire qui a déjà pris acte de la disparition de la majorité des nègres. 
Je dis qu'aucun discours, aucune proclamation sur la culture ne nous détourneront de nos tâches fondamentales qui sont la libération du territoire national, une lutte de tous les instants contre les formes nouvelles du colonialisme, un refus obstiné de nous entr'émerveiller au sommet. 
[225]

\section{LES DAMNÉS DE LA TERRE}

\section{IV}

\section{Fondements réciproques de la culture nationale et des luttes de libération}

\section{Retour à la table des matières}

La domination coloniale, parce que totale et simplifiante, a tôt fait de disloquer de façon spectaculaire l'existence culturelle du peuple soumis. La négation de la réalité nationale, les rapports juridiques nouveaux introduits par la puissance occupante, le rejet à la périphérie par la société coloniale des indigènes et de leurs coutumes, l'expropriation, l'asservissement systématisé des hommes et des femmes rendent possible cette oblitération culturelle.

J'ai montré il y a trois ans, devant notre premier congrès, qu'assez rapidement, dans la situation coloniale, le dynamisme est remplacé par une substantification des attitudes. L'aire culturelle est alors délimitée par des garde-fous, des poteaux indicateurs. Ce sont autant de mécanismes de défense du type le plus élémentaire, assimilables à plus 
d'un titre au simple instinct de conservation. L'intérêt de cette période est que l'oppresseur en arrive à ne plus se satisfaire de l'inexistence objective de la nation et de la culture opprimées. Tous les efforts sont faits pour amener le colonisé à confesser l'infériorité de sa culture transformée en conduites instinctives, à reconnaître l'irréalité de sa nation et, à l'extrême, le caractère inorganisé et non fini de sa propre structure biologique.

Face à cette situation, la réaction du colonisé n'est pas univoque. Tandis que les masses maintiennent intactes les traditions les plus hétérogènes à la situation coloniale, tandis que le style artisanal se solidifie dans un formalisme de plus en plus [226] stéréotypé, l'intellectuel se jette frénétiquement dans l'acquisition forcenée de la culture de l'occupant en prenant soin de caractériser péjorativement sa culture nationale, ou se cantonne dans l'énumération circonstanciée, méthodique, passionnelle et rapidement stérile de cette culture.

Le caractère commun de ces deux tentatives est qu'elles débouchent l'une et l'autre sur des contradictions insupportables. Transfuge ou substantialiste, le colonisé est inefficace parce que précisément l'analyse de la situation coloniale n'est pas menée avec rigueur. La situation coloniale arrête, dans sa quasi-totalité, la culture nationale. Il n'y a pas, il ne saurait y avoir de culture nationale, de vie culturelle nationale, d'inventions culturelles ou de transformations culturelles nationales dans le cadre d'une domination coloniale. Çà et là surgissent quelquefois des tentatives hardies de réamorcer le dynamisme culturel, de réorienter les thèmes, les formes, les tonalités. L'intérêt immédiat, palpable, évident de ces soubresauts est nul. Mais, en en poursuivant les conséquences jusqu'à leur extrême limite, on s'aperçoit que se prépare une désopacification de la conscience nationale, une mise en question de l'oppression, une ouverture sur la lutte de libération.

La culture nationale est, sous la domination coloniale, une culture contestée et dont la destruction est poursuivie de façon systématique. 
C'est très rapidement une culture condamnée à la clandestinité. Cette notion de clandestinité est immédiatement perçue dans les réactions de l'occupant qui interprète la complaisance dans les traditions comme une fidélité à l'esprit national, comme un refus de se soumettre. Cette persistance dans des formes culturelles condamnées par la société coloniale est déjà une manifestation nationale. Mais cette manifestation renvoie aux lois de l'inertie. Il n'y a pas d'offensive, pas de redéfinition des rapports. Il y a crispation sur un noyau de plus en plus étriqué, de plus en plus inerte, de plus en plus vide.

Au bout d'un ou deux siècles d'exploitation se produit une véritable émaciation du panorama culturel national. La culture nationale devient un stock d'habitudes motrices, de traditions vestimentaires, d'institutions morcelées. On y décèle peu de mobilité. Il n'y a pas de créativité vraie, pas d'effervescence. Misère du peuple, oppression nationale et inhibition de la culture sont une seule et même chose. Après un siècle de domination coloniale on trouve une culture rigidifiée à l'extrême, sédimentée, minéralisée. Le dépérissement de la réalité nationale et l'agonie de la culture nationale entretiennent des rapports de dépendance réciproque. C'est pourquoi il devient capital de suivre l'évolution de ces rapports au cours de la lutte de libération. La négation culturelle, le mépris des manifestations nationales motrices ou émotionnelles, la mise hors la loi de toute spécialité d'organisation contribuent à engendrer des conduites agressives chez le colonisé. Mais ces conduites sont de type réflexe, mal différenciées, anarchiques, inefficaces. L'exploitation coloniale, la misère, la famine endémique acculent de plus en plus le colonisé à la lutte ouverte et organisée. Progressivement et de façon imperceptible la nécessité d'un affrontement décisif se fait prégnante et est ressentie par la grande majorité du peuple. Les tensions, inexistantes auparavant, se multiplient. Les événements internationaux, l'écroulement, par pans entiers, des empires coloniaux, les contradictions inhérentes au système colonialiste entretiennent et renforcent la combativité, promeuvent et donnent force à la conscience nationale. 
Ces tensions nouvelles, présentes à tous les étages de la réalité coloniale, se répercutent sur le plan culturel. En littérature par exemple il y a surproduction relative. De réplique mineure du dominateur qu'elle était, la production autochtone se différencie et se fait volonté particularisante. Essentiellement consommatrice pendant la période d'oppression, l'intelligentzia devient productive. Cette littérature se cantonne d'abord volontiers dans le genre poétique et tragique. Par la suite seront abordés les [228] romans, les nouvelles et les essais. Il semble exister une sorte d'organisation interne, une loi de l'expression qui veut que les manifestations poétiques se raréfient à mesure que se précisent les objectifs et les méthodes de la lutte de libération. Les thèmes sont fondamentalement renouvelés. De fait, on trouve de moins en moins ces récriminations amères et désespérées, ces violences épanouies et sonores qui, somme toute, tranquillisent l'occupant. Les colonialistes ont, dans la période antérieure, encouragé ces tentatives, leur ont facilité l'existence. Les dénonciations acérées, les misères étalées, la passion exprimée sont, en effet, assimilées par l'occupant à une opération cathartique. Faciliter ces opérations c'est, en un certain sens, éviter la dramatisation, détendre l'atmosphère.

Mais cette situation ne peut être que transitoire. En effet, le progrès de la conscience nationale dans le peuple modifie et précise les manifestations littéraires de l'intellectuel colonisé. La cohésion continuée du peuple constitue pour l'intellectuel une invitation à dépasser le cri. La plainte fait face au réquisitoire puis à l'appel. À la période suivante apparaît le mot d'ordre. La crisallisation de la conscience nationale va à la fois bouleverser les genres et les thèmes littéraires et créer de toutes pièces un nouveau public. Alors qu'au début l'intellectuel colonisé produisait à l'intention exclusive de l'oppresseur, soit pour le charmer, soit pour le dénoncer à travers des catégories ethniques ou subjectivistes, il adopte progressivement l'habitude de s'adresser à son peuple. 
C'est seulement à partir de ce moment que l'on peut parler de littérature nationale. Il y a, au niveau de la création littéraire, reprise et clarification des thèmes typiquement nationalistes. C'est la littérature de combat proprement dite, en ce sens qu'elle convoque tout un peuple à la lutte pour l'existence nationale. Littérature de combat, parce qu'elle informe la conscience nationale, lui donne forme et contours et lui ouvre de nouvelles et d'illimitées perspectives. Littérature de combat, parce qu'elle prend en charge, parce qu'elle est volonté temporalisée.

[229]

$\grave{A}$ un autre niveau, la littérature orale, les contes, les épopées, les chants populaires autrefois répertoriés et figés commencent à se transformer. Les conteurs qui récitaient des épisodes inertes les animent et $y$ introduisent des modifications de plus en plus fondamentales. Il y a tentative d'actualiser les conflits, de moderniser les formes de lutte évoquées, les noms des héros, le type des armes. La méthode allusive se fait de plus en plus fréquente. À la formule: «Il y a très longtemps de cela » on substitue celle plus ambiguë: «Ce qui va être rapporté s'est passé quelque part mais cela aurait pu se passer ici aujourd'hui ou demain. » L'exemple de l'Algérie est, à cet égard, significatif. À partir de 1952-1953, les conteurs, stéréotypés et fatigants à écouter, bouleversent de fond en comble et leurs méthodes d'exposé et le contenu de leurs récits. Le public, autrefois clairsemé, se fait compact. L'épopée, avec ses catégories de typification, reparaît. C'est un authentique spectacle qui reprend valeur culturelle. Le colonialisme ne s'y est pas trompé qui, à partir de 1955, a procédé à l'arrestation systématique de ces conteurs.

Le contact du peuple avec la geste nouvelle suscite un nouveau rythme respiratoire, des tensions musculaires oubliées et développe l'imagination. Chaque fois que le conteur expose devant son public un épisode nouveau, on assiste à une réelle invocation. Il est révélé au public l'existence d'un nouveau type d'homme. Le présent n'est plus 
fermé sur lui-même mais écartelé. Le conteur redonne liberté à son imagination, innove, fait cuvre créatrice. Il arrive même que des figures mal préparées à cette transmutation, bandits de grands chemins ou vagabonds plus ou moins asociaux, soient reprises et remodelées. Il faut suivre pas à pas dans un pays colonisé l'émergence de l'imagination, de la création dans les chansons et dans les récits épiques populaires. Le conteur répond par approximations successives à l'attente du peuple et chemine, apparemment solitaire, mais en réalité soutenu par l'assistance, à la recherche de modèles nouveaux, de modèles nationaux. La comédie et la farce disparaissent ou perdent leur attrait. Quant à la dramatisation, elle ne se [230] situe plus au niveau de la conscience en crise de l'intellectuel. En perdant ses caractères de désespoir et de révolte, elle est devenue le lot commun du peuple, elle est devenue partie d'une action en préparation ou déjà en cours.

Sur le plan artisanal, les formes sédimentées et comme frappées de stupeur progressivement se tendent. Le travail du bois par exemple, qui rééditait par milliers certains visages ou certaines poses, se différencie. Le masque inexpressif ou accablé s'anime et les bras ont tendance à quitter le corps, à esquisser l'action. La composition à deux, trois, cinq personnages apparaît. Les écoles traditionnelles sont invitées à la création par le surgissement en avalanche d'amateurs ou de dissidents. Cette vigueur nouvelle dans ce secteur de la vie culturelle passe très souvent inaperçue. Pourtant sa contribution à la lutte nationale est capitale. En animant visages et corps, en prenant comme thème de création un groupe vissé sur un même socle l'artiste convie au mouvement organisé.

Si l'on étudie les répercussions de l'éveil de la conscience nationale dans le domaine de la céramique ou de la poterie, les mêmes observations peuvent être signalées. Les créations abandonnent leur formalisme. Cruches, jarres, plateaux sont modifiés, d'abord de façon imperceptible puis de façon brutale. Les coloris, autrefois en nombre 
restreint et qui obéissaient à des lois harmoniques traditionnelles, se multiplient et subissent le contrecoup de la poussée révolutionnaire. Certains ocres, certains bleus, interdits semble-t-il de toute éternité au sein d'une aire culturelle donnée, s'imposent sans scandale. De même la non-figuration du visage humain, caractéristique selon les sociologues des régions parfaitement délimitées, devient soudain tout à fait relative. Le spécialiste métropolitain, l'ethnologue perçoivent rapidement ces mutations. Dans l'ensemble, toutes ces mutations sont condamnées au nom d'un style artistique codifié, d'une vie culturelle se développant au sein de la situation coloniale. Les spécialistes colonialistes ne [231] reconnaissent pas cette forme nouvelle et accourent au secours des traditions de la société autochtone. Ce sont les colonialistes qui se font les défenseurs du style indigène. On se souvient parfaitement, et l'exemple revêt une certaine importance parce qu'il ne s'agit pas tout à fait d'une réalité coloniale, des réactions des spécialistes blancs du jazz quand, après la Deuxième Guerre mondiale, se cristallisèrent de façon stable de nouveaux styles comme le be-bop. C'est que le jazz ne doit être que la nostalgie cassée et désespérée d'un vieux nègre pris entre cinq whiskies, sa propre malédiction et la haine raciste des Blancs. Dès lors que le nègre s'appréhende et appréhende le monde différemment, fait naître l'espoir et impose un recul à l'univers raciste, il est clair que sa trompette tend à se déboucher et sa voix à se désenrouer. Les nouveaux styles en matière de jazz ne sont pas seulement nés de la concurrence économique. Il faut y voir à n'en pas douter une des conséquences de la défaite, inéluctable quoique lente, de l'univers sudiste aux États-Unis. Et il n'est pas utopique de supposer que dans une cinquantaine d'années la catégorie jazz-cri hoqueté d'un pauvre nègre maudit sera défendue par les seuls Blancs, fidèles à l'image stoppée d'un type de rapports, d'une forme de la négritude.

On pourrait également rechercher et trouver, au niveau de la danse, du chant mélodique, des rites, des cérémonies traditionnelles la même poussée, déceler les mêmes mutations, la même impatience. Bien avant la phase politique ou armée de la lutte nationale, un lecteur at- 
tentif peut donc sentir et voir se manifester la vigueur nouvelle, le combat prochain. Formes d'expression inaccoutumées, thèmes inédits et doués d'un pouvoir non plus d'invocation niais de rassemblement, de convocation « en vue de». Tout concourt à réveiller la sensibilité du colonisé, à rendre inactuelles, inacceptables les attitudes contemplatives ou d'échec. Parce qu'il renouvelle les intentions et la dynamique de l'artisanat, de la danse et de la musique, de la littérature et de l'épopée orale, le colonisé restructure sa perception. Le monde [232] perd son caractère maudit. Les conditions sont réunies pour l'inévitable confrontation.

Nous avons assisté à l'apparition du mouvement dans les manifestations culturelles. Nous avons vu que ce mouvement, ces nouvelles formes étaient liées à la maturation de la conscience nationale. Or, ce mouvement tend de plus en plus à s'objectiver, à s'institutionnaliser. D'où la nécessité d'une existence nationale coûte que coûte.

L'une des erreurs, difficilement soutenable d'ailleurs, est de tenter des inventions culturelles, de revaloriser la culture autochtone dans le cadre de la domination coloniale. C'est pourquoi nous aboutissons à une proposition d'allure paradoxale: dans un pays colonisé le nationalisme le plus élémentaire, le plus brutal, le plus indifférencié est la forme la plus fervente et la plus efficace de défense de la culture nationale. La culture est d'abord expression d'une nation, de ses préférences, de ses interdits, de ses modèles. C'est à tous les étages de la société globale que se constituent d'autres interdits, d'autres valeurs, d'autres modèles. La culture nationale, c'est la somme de toutes ces appréciations, la résultante des tensions internes et externes à la société globale et aux différentes couches de cette société. Dans la situation coloniale, la culture privée du double support de la nation et de l'État dépérit et agonise. La condition d'existence de la culture est donc la libération nationale, la renaissance de l'État. 
La nation n'est pas seulement condition de la culture, de son effervescence, de son renouvellement continué, de son approfondissement. Elle est aussi une exigence. C'est d'abord le combat pour l'existence nationale qui débloque la culture, lui ouvre les portes de la création. C'est plus tard la nation qui assurera à la culture les conditions, le cadre d'expression. La nation réunit à l'intention de la culture les différents éléments indispensables et qui seuls peuvent lui conférer crédibilité, [233] validité, dynamisme, créativité. C'est également son caractère national qui rendra la culture perméable aux autres cultures et lui permettra d'influencer, de pénétrer d'autres cultures. Ce qui n'existe pas ne peut guère agir sur le réel, ni même influencer ce réel. Il faut d'abord que le rétablissement de la nation donne vie, au sens le plus biologique du terme, à la culture nationale.

Nous avons donc suivi le craquement de plus en plus essentiel des vieilles sédimentations culturelles et saisi, à la veille du combat décisif pour la libération nationale, le renouveau de l'expression, le démarrage de l'imagination.

Il reste qu'ici une question fondamentale se pose. Quels sont les rapports qui existent entre la lutte, le conflit - politique ou armé - et la culture? Durant le conflit, y a-t-il suspension de la culture? La lutte nationale est-elle une manifestation culturelle? Faut-il enfin dire que le combat libérateur, quoique fécond a posteriori pour la culture, est en lui-même une négation de la culture? La lutte de libération estelle, oui ou non, un phénomène culturel?

Nous pensons que la lutte organisée et consciente entreprise par un peuple colonisé pour rétablir la souveraineté de la nation constitue la manifestation la plus pleinement culturelle qui soit. Ce n'est pas uniquement le succès de la lutte qui donne par la suite validité et vigueur à la culture, il n'y a pas mise en hibernation de la culture pendant le combat. La lutte elle-même, dans son déroulement, dans son processus 
interne développe les différentes directions de la culture et en esquisse de nouvelles. La lutte de libération ne restitue pas à la culture nationale sa valeur et ses contours anciens. Cette lutte qui vise à une redistribution fondamentale des rapports entre les hommes ne peut laisser intacts ni les formes ni les contenus culturels de ce peuple. Après la lutte il n'y a pas seulement disparition du colonialisme mais aussi disparition du colonisé.

[234]

Cette nouvelle humanité, pour soi et pour les autres, ne peut pas ne pas définir un nouvel humanisme. Dans les objectifs et les méthodes de la lutte est préfiguré ce nouvel humanisme. Un combat qui mobilise toutes les couches du peuple, qui exprime les intentions et les impatiences du peuple qui ne craint pas de s'appuyer presque exclusivement sur ce peuple, est nécessairement triomphant. La valeur de ce type de combat est qu'il réalise le maximum de conditions pour le développement et l'invention culturels. Après la libération nationale obtenue dans ces conditions, il n'y a pas cette indécision culturelle si pénible que l'on trouve dans certains pays nouvellement indépendants. C'est que la nation dans sa forme de venue au monde, dans ses modalités d'existence influe fondamentalement sur la culture. Une nation née de l'action concertée du peuple, qui incarne les aspirations réelles du peuple, qui modifie l'État ne peut exister que sous des formes de fécondité culturelle exceptionnelle.

Les colonisés qui s'inquiètent de la culture de leur pays et qui veulent lui donner dimension universelle ne doivent donc pas faire confiance au seul principe de l'indépendance inévitable et sans inscription dans la conscience du peuple pour réaliser cette tâche. La libération nationale comme objectif est une chose, les méthodes et le contenu populaire du combat en sont une autre. Il nous semble que les lendemains de la culture, la richesse d'une culture nationale sont fonction également des valeurs qui ont hanté le combat libérateur. 
Et voici venu le moment de dénoncer le pharisaïsme de certains. La revendication nationale, dit-on çà et là, est une phase que l'humanité a dépassée. L'heure est aux grands ensembles et les attardés du nationalisme doivent en conséquence corriger leurs erreurs. Nous pensons au contraire que l'erreur, lourde de conséquences, consisterait à vouloir sauter l'étape nationale. Si la culture est la manifestation de la conscience nationale, je n'hésiterai pas à dire, dans le cas qui nous occupe, que la conscience nationale est la forme la plus élaborée de la culture.

[235]

La conscience de soi n'est pas fermeture à la communication. La réflexion philosophique nous enseigne au contraire qu'elle en est la garantie. La conscience nationale, qui n'est pas le nationalisme, est la seule à nous donner dimension internationale. Ce problème de la conscience nationale, de la culture nationale prend en Afrique des dimensions particulières. La naissance de la conscience nationale en Afrique entretient avec la conscience africaine des relations de stricte contemporanéité. La responsabilité de l'Africain devant sa culture nationale est aussi responsabilité devant la culture négro-africaine. Cette responsabilité conjointe n'est pas le fait d'un principe métaphysique mais la conscience d'une loi banale qui veut que toute nation indépendante, dans une Afrique où le colonialisme demeure accroché, soit une nation encerclée, fragile, en danger permanent.

Si l'homme est ce qu'il fait, alors nous dirons que la chose la plus urgente aujourd'hui pour l'intellectuel africain est la construction de sa nation. Si cette construction est vraie, c'est-à-dire si elle traduit le vouloir manifeste du peuple, si elle révèle dans leur impatience les peuples africains, alors la construction nationale s'accompagne nécessairement de la découverte et de la promotion de valeurs universalisantes. Loin donc de s'éloigner des autres nations, c'est la libération nationale qui rend la nation présente sur la scène de l'histoire. C'est au cœur de la conscience nationale que s'élève et se vivifie la conscience internationale. Et cette double émergence n'est, en définitive, que le foyer de toute culture. 
Communication faite au deuxième Congrès des écrivains et artistes noirs, Rome, 1959. 
[237]

LES DAMNÉS DE LA TERRE

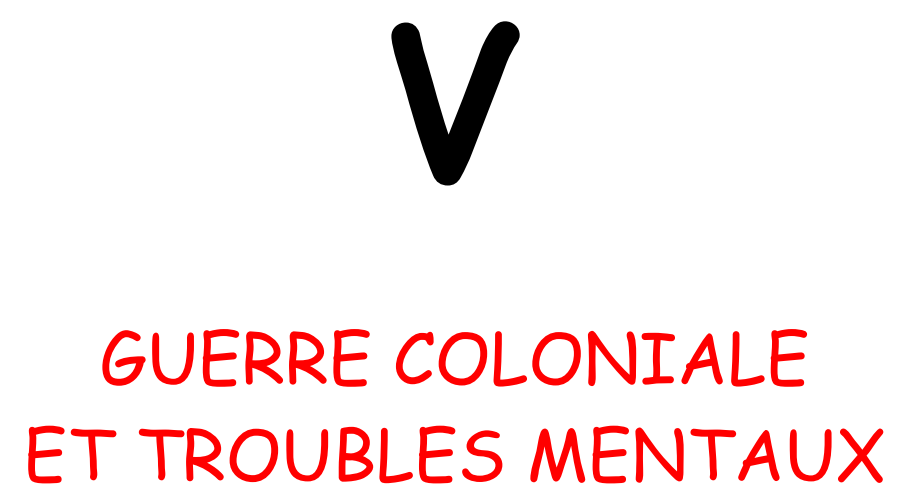

Retour à la table des matières 
Mais la guerre continue. Et nous aurons à panser des années encore les plaies multiples et quelquefois indélébiles faites à nos peuples par le déferlement colonialiste.

L'impérialisme, qui aujourd'hui se bat contre une authentique libération des hommes, abandonne çà et là des germes de pourriture qu'il nous faut implacablement détecter et extirper de nos terres et de nos cerveaux.

Nous abordons ici le problème des troubles mentaux nés de la guerre de libération nationale que mène le peuple algérien.

On trouvera peut-être inopportunes et singulièrement déplacées dans un tel livre ces notes de psychiatrie. Nous n'y pouvons strictement rien.

Il n'a pas dépendu de nous que dans cette guerre des phénomènes psychiatriques, des troubles du comportement et de la pensée aient pris de l'importance chez les acteurs de la «pacification» ou au sein de la population « pacifiée». La vérité est que la colonisation, dans son essence, se présentait déjà comme une grande pourvoyeuse des hôpitaux psychiatriques. Dans différents travaux scientifiques nous avons, depuis 1954, attiré l'attention des psychiatres français et internationaux sur la difficulté qu'il y avait à « guérir » correctement un colonisé, c'est-à-dire à le rendre homogène de part en part à un milieu social de type colonial. 
Parce qu'il est une négation systématisée de l'autre, une décision forcenée de refuser à l'autre tout attribut d'humanité, le colonialisme accule le peuple dominé à se poser constamment la question: «Qui suis-je en réalité ? »

Les positions défensives nées de cette confrontation violente du colonisé et du système colonial s'organisent en une structure qui révèle alors la personnalité colonisée. Il suffit, pour comprendre cette « sensitivité », simplement d'étudier, d'apprécier le nombre et la profondeur des blessures faites à un colonisé pendant une seule journée passée au sein du régime colonial. Il faut se souvenir en tout cas qu'un peuple colonisé n'est pas seulement un peuple dominé. Sous l'occupation allemande les Français étaient demeurés des hommes. Sous l'occupation française, les Allemands sont demeurés des hommes. En Algérie, il n'y a pas seulement domination, mais à la lettre décision de n'occuper somme toute qu'un terrain. Les Algériens, les femmes en « haïk», les palmeraies et les chameaux forment le panorama, la toile de fond naturelle de la présence humaine française.

La nature hostile, rétive, foncièrement rebelle est effectivement représentée aux colonies par la brousse, les moustiques, les indigènes et les fièvres. La colonisation est réussie quand toute cette nature indocile est enfin matée. Chemins de fer à travers la brousse, assèchement des marais, inexistence politique et économique de l'indigénat sont en réalité une seule et même chose.

Dans la période de colonisation non contestée par la lutte armée, quand la somme d'excitations nocives dépasse un certain seuil, les positions défensives des colonisés s'écroulent, et ces derniers se retrouvent alors en nombre important dans les hôpitaux psychiatriques. Il y a donc dans cette période calme de colonisation réussie une régulière et importante pathologie mentale produite directement par l'oppression.

Aujourd'hui la guerre de libération nationale que mène le peuple algérien depuis sept ans, parce qu'elle est totale chez le peuple, est de- 
venue un terrain favorable à l'éclosion des troubles [241] mentaux 20. Nous mentionnons ici quelques cas de malades algériens et français, soignés par nous et qui nous paraissent particulièrement parlants. Nous ne livrons pas, il est superflu de le mentionner, un travail scientifique. Nous évitons toute discussion séméiologique, nosologique ou thérapeutique. Les quelques termes techniques utilisés ici servent uniquement de repères. Il nous faut toutefois insister sur deux points.

En règle générale, la psychiatrie clinique range les différents troubles présentés par nos malades sous la rubrique de «psychoses réactionnelles». Ce faisant, on privilégie l'événement qui a déclenché la maladie quoique, çà et là, soient mentionnés le rôle du terrain (l'histoire psychologique, affective et biologique du sujet) et celui du milieu. Il nous semble que, dans les cas présentés ici, l'événement déclenchant est principalement l'atmosphère sanglante, impitoyable, la généralisation de pratiques inhumaines, l'impression tenace qu'ont les gens d'assister à une véritable apocalypse.

Le cas numéro 2 de la série $A$ est typiquement une psychose réactionnelle, mais les cas numéros $1,2,4,5$ de la série $B$ admettent une causalité beaucoup plus diffuse sans qu'on puisse véritablement parler d'un événement déclenchant particulier. Ici, c'est la guerre, c'est cette guerre coloniale qui très souvent prend l'allure d'un authentique génocide, cette guerre enfin qui [242] bouleverse et casse le monde, qui est l'événement déclenchant. Psychose réactionnelle, si l'on veut utili-

20 Dans l'introduction non publiée dans les deux premières éditions de L'An $V$ de la révolution algérienne, nous signalions déjà que toute une génération d'Algériens, baignée dans l'homicide gratuit et collectif avec les conséquences psychoaffectives que cela entraîne, serait l'héritage humain de la France en Algérie. Les hommes français qui condamnent la torture en Algérie adoptent constamment un point de vue strictement français. Ce n'est pas un reproche, c'est une constatation: on veut protéger la conscience des tortionnaires actuels et en puissance et l'on tente d'éviter le pourrissement moral de la jeunesse française. Nous ne pouvons quant à nous qu'être d'accord avec cette démarche. Certaines observations réunies ici, principalement les cas numéros 4 et 5 de la série $A$ illustrent et justifient tristement cette hantise des démocrates français. Notre propos, en tout cas, est de montrer que la torture subie disloque très profondément on s'en serait douté, la personnalité du torturé. 
ser une étiquette déjà établie, mais en accordant ici une singulière priorité à la guerre prise dans sa totalité et dans ses particularités de guerre coloniale. Après les deux grandes guerres mondiales, les publications n'ont pas fait défaut sur la pathologie mentale des militaires engagés dans l'action et des civils victimes de l'exode ou des bombardements. La physionomie inédite de certains tableaux psychiatriques signalés ici confirme, s'il en est encore besoin, que cette guerre coloniale est originale même dans la pathologie qu'elle sécrète.

Une autre notion fortement établie mérite à nos yeux un léger assouplissement: il s'agit de la relative bénignité de ces troubles réactionnels. Et, certes, on a pu décrire, mais de façon toujours exceptionnelle, des psychotisations secondaires, c'est-à-dire des cas où l'ensemble de la personnalité est définitivement disloqué. Il nous semble au contraire que la règle est ici la fréquente malignité des processus pathologiques. Ce sont des troubles qui persistent des mois durant, attaquant massivement le moi, et laissant presque toujours comme séquelle une fragilité pratiquement discernable à vue d'œil. De toute évidence, l'avenir de ces malades est hypothéqué. Un exemple illustrera notre point de vue.

Dans un des pays africains indépendants depuis plusieurs années, nous avons eu l'occasion de recevoir un patriote, ancien résistant. Cet homme d'une trentaine d'années venait nous demander conseil et soulagement, car à l'approche d'une certaine date de l'année, des insomnies s'installaient, accompagnées d'anxiété et d'idées fixes d'autodestruction. La date critique était celle où, sur instruction de son réseau, il avait posé quelque part une bombe. Dix personnes avaient trouvé la mort au cours de l'attentat 21.

21 Les circonstances d'apparition de ces troubles sont intéressantes à plus d'un titre. Plusieurs mois après l'indépendance de son pays, il avait fait la connaissance de ressortissants de l'ancienne nation occupante. Il les avait trouvés sympathiques. Ces hommes et ces femmes saluaient l'indépendance acquise et rendaient hommage sans réserve au courage des patriotes dans la lutte de libération nationale. Ce militant eut alors une sorte de vertige. Il se demanda avec angoisse si parmi les victimes de la bombe il aurait pu se trouver des gens semblables à 
[243]

Ce militant, qui à aucun moment n'envisageait de renier son action passée, savait de façon très claire le prix que sa personne avait dû payer pour l'indépendance nationale. De tels cas limites posent le problème de la responsabilité dans le cadre révolutionnaire.

Les observations que nous citons ici couvrent la période qui va de 1954 à 1959. Certains malades ont été vus en Algérie, soit dans les centres hospitaliers, soit en clientèle. Les autres ont été soignés dans les formations sanitaires de l'Armée de libération nationale.

ses interlocuteurs. Certes, le café visé était un repaire de racistes notoire, mais rien n'interdisait à un quelconque passant d'y rentrer et de consommer. À partir du jour où il eut ce premier vertige, l'homme essaya d'éviter de penser aux événements anciens. Or, paradoxalement, quelques jours avant la date critique, les premiers troubles apparaissaient. Depuis, ils se répètent très régulièrement.

Autrement dit, nos actes ne cessent jamais de nous poursuivre. Leur arrangement, leur mise en ordre, leur motivation peuvent parfaitement a posteriorise trouver profondément modifiés. Ce n'est pas l'un des moindres pièges que nous tend l'Histoire et ses multiples déterminations. Mais pouvons-nous échapper au vertige? Qui oserait prétendre que le vertige ne hante pas toute existence? 


\section{LES DAMNÉS DE LA TERRE}

\section{$\mathbf{V}$ \\ Série A}

Cinq cas sont rassemblés ici. Il s'agit d'Algériens ou d'Européens ayant présenté, après des faits bien précis, des troubles mentaux de type réactionnel.

\section{CAS No 1. \\ Impuissance chez un algérien consécutive au viol de sa femme}

\section{Retour à la table des matières}

B... est un homme de 26 ans. Il nous est adressé par le Service sanitaire du Front de libération nationale pour migraines rebelles et insomnies. Ancien chauffeur de taxi, a milité depuis l'âge de 18 ans dans les partis nationalistes. À partir de 1955, devient membre d'une cellule FLN. À plusieurs occasions, il utilise son taxi pour le transport de tracts et de responsables politiques. Devant l'aggravation de la répression, le FLN décide de porter la guerre dans les centres urbains, B... est alors amené à conduire des commandos à proximité des points d'attaque, et assez souvent à les attendre. 
Un jour, cependant, en pleine ville européenne, après une action relativement importante, un bouclage extrêmement sérieux l'oblige à abandonner son taxi et, en ordre dispersé, le commando se, disloque. B... qui arrive à échapper au dispositif de l'adversaire, se réfugie chez un ami, et quelques jours après, sans avoir reparu à son domicile, gagne sur instruction de ses responsables le maquis le plus proche.

Pendant plusieurs mois, il va être sans nouvelles de sa femme et de sa petite fille âgée de vingt mois. Il apprendra par contre que la police l'a, des semaines entières, recherché dans la [245] ville. Après deux ans de séjour au maquis, il reçoit de sa femme un message où elle lui demande de l'oublier. Elle est déshonorée. Il ne doit plus envisager de reprendre la vie commune avec elle. Terriblement inquiet, il demande à son commandant l'autorisation de se rendre clandestinement à son domicile. Ce qui lui est refusé. Par contre, des mesures sont prises pour qu'un membre du FLN contacte la femme et les parents de B...

Deux semaines après, un rapport détaillé arrive au commandant de l'unité de $B$...

Sitôt après la découverte de son taxi abandonné (deux chargeurs de mitraillette y avaient été trouvés) des soldats français accompagnés de policiers s'étaient rendus à son domicile. Le trouvant absent, ils emmenèrent sa femme qu'ils gardèrent pendant plus d'une semaine.

Elle est interrogée sur les fréquentations de son mari, et pendant deux jours assez brutalement giflée. Mais le troisième jour, un militaire français - elle est incapable de préciser si c'est un officier - fait sortir les autres et la viole. Quelque temps après, un deuxième, en présence cette fois des autres, la viole en lui disant: «Si tu revois un jour ton salaud de mari, n'oublie surtout pas de lui dire ce qu'on t'a fait. " Elle reste encore une semaine sans subir de nouvel interrogatoire. Après quoi on la raccompagne à son domicile. Ayant raconté son histoire à sa mère, celle-ci la convainc de tout dire à B... Aussi, dès le premier contact établi avec son mari, lui avoue-t-elle son déshonneur.

Le premier choc passé, engagé par ailleurs dans une action de tous les instants, B... reprend le dessus. Pendant plusieurs mois il entend de 
multiples récits de femmes algériennes violées ou torturées; il aura l'occasion de voir des maris de femmes violées et ses malheurs personnels, sa dignité de mari bafoué restent au second plan.

En 1958, il est chargé d'une mission à l'extérieur. Au moment de rejoindre son unité, une distractivité inhabituelle et des insomnies inquiètent ses camarades et ses supérieurs. Son départ est retardé, et une visite médicale décidée. C'est à ce [246] moment que nous le voyons. Bon contact immédiat. Visage mobile, un peu trop peut-être. Les sourires sont légèrement exagérés. Euphorie de surface : « Ça va... ça va... Je me sens mieux maintenant. Donne-moi quelques fortifiants, des vitamines, et laisse-moi remonter. " Par en dessous perce une anxiété de base. Est hospitalisé aussitôt.

Dès le deuxième jour, l'optimisme-écran s'effondre, et c'est un déprimé pensif, anorexique, qui garde le lit, que nous avons devant nous. Il fuit les discussions politiques et manifeste un désintérêt marqué pour tout ce qui concerne la lutte nationale. Il évite d'écouter les nouvelles ayant trait à la guerre de libération. L'abord de ses difficultés est très laborieux, mais au bout de quelques jours, nous pouvons reconstituer son histoire.

Pendant son séjour à l'extérieur, tente une aventure sexuelle qui échoue. Pensant qu'il s'agit de fatigue, normale après des marches forcées et des périodes de sous-alimentation, recommence deux semaines plus tard. Nouvel échec. En parle à un camarade qui lui conseille de la vitamine B 12. En prend sous forme de comprimés. Nouvelle tentative, nouvel échec. De plus, quelques instants avant l'acte, envie irrésistible de déchirer une photo de sa petite fille. Une telle liaison symbolique pouvait évoquer l'existence de pulsions incestueuses inconscientes. Cependant, plusieurs entretiens et un rêve (le malade assiste à la rapide putréfaction d'un petit chat avec dégagement d'odeurs insupportables) nous conduisent dans une tout autre direction. « Cette fille, nous dit-il un jour (il s'agit de sa petite fille), a quelque chose de pourri en elle. » À partir de cette période, les insomnies deviennent très pénibles, et malgré une dose assez importante de neuroleptiques se développe un état d'excitation anxieuse qui trouble considérablement le Service. Il 
nous parle alors pour la première fois de sa femme en riant et nous dit : «Elle a goûté du Français. » C'est à ce moment que nous reconstituons toute l'histoire. La trame des événements est explicitée. Il nous apprend qu'avant chaque tentative sexuelle il pense à sa femme. Toutes ses confidences nous paraissent d'un intérêt fondamental.

« Je me suis marié avec cette fille alors que j'aimais ma cousine. Mais les parents de la cousine ont arrangé le mariage de leur fille avec quelqu'un d'autre. Alors j'ai accepté la première femme que mes parents m'ont proposée. Elle était gentille, mais je ne l'aimais pas. Je me disais toujours: tu es jeune... attends un peu, et quand tu trouveras le bon numéro, tu divorceras et tu feras un bon mariage. Aussi étais-je peu attaché à ma femme. Avec les événements, je me suis éloigné d'elle encore davantage. À la fin, je venais prendre mes repas et dormir sans presque lui parler.

« Au maquis, quand j'ai appris qu'elle avait été violée par des Français, j'ai d'abord ressenti de la colère contre ces salauds Puis j'ai dit: "Oh, ce n'est pas grave; elle n'a pas été tuée. Elle pourra recommencer sa vie." Et plusieurs semaines après, je me suis rendu compte qu'elle avait été violée parce qu'on me recherchait. En fait, c'est pour la punir de son silence qu'elle fut violée. Elle aurait parfaitement pu indiquer au moins un nom de militant à partir duquel on pouvait retrouver, détruire le réseau, et peut-être même marrêter. Ce n'était donc pas un simple viol, par désœuvrement ou par sadisme comme j'ai eu l'occasion d'en voir dans les douars, c'était le viol d'une femme têtue, qui acceptait tout au lieu de vendre son mari. Et ce mari, c'était moi. Cette femme m'avait sauvé la vie, et avait protégé le réseau. C'était à cause de moi qu'elle était déshonorée. Pourtant elle ne me disait pas: "Voici ce que j'ai enduré pour toi." Elle me disait au contraire: "Oublie-moi, refais ta vie, je suis déshonorée."

«C'est à partir de ce moment que j'ai décidé en moi-même de reprendre ma femme après la guerre, car il faut te dire que j'ai vu des paysans essuyer les larmes de leurs femmes qui avaient été violées 
sous leurs yeux. Cela m'a beaucoup ébranlé. Je dois t'avouer d'ailleurs qu'au début je ne pouvait comprendre leur attitude. Mais, de plus en plus, nous avons été amenés à intervenir dans ces histoires pour expliquer aux civils. J'ai vu des civils volontaires pour épouser une jeune fille violée par les militaires français et devenue enceinte. Tout cela m'a amené à repenser le problème de ma femme.

[248]

« J'ai décidé de la reprendre, mais j'ignore encore comment je réagirai en la voyant. Et souvent, en regardant la photo de ma fille, je pense qu'elle aussi est déshonorée. Comme si tout ce qui venait de ma femme était pourri. S'ils l'avaient torturée, s'ils lui avaient brisé toutes les dents, cassé un bras, cela ne maurait rien fait. Mais cette chose, est-ce qu'on peut l'oublier? Et était-elle obligée de me mettre au courant de tout cela?»

Il me demande alors si sa « faiblesse sexuelle » est à mon avis causée par ses tracas.

Réponse: «Cela n'est pas impossible. »

Il s'assied alors sur le lit :

«Qu'est-ce que tu ferais si cela t'arrivait?

- Je ne sais pas...

- Reprendrais-tu ta femme?

- Je pense que oui...

- Ah, tu vois... Tu n'es pas tout à fait sûr... »

Se prend la tête dans les mains et après quelques instants quitte la chambre.

À partir de ce jour, accepte progressivement d'entendre des discussions politiques, tandis que les migraines et l'anorexie régressent considérablement.

Au bout de deux semaines, rejoint son unité en me disant. 
«À l'indépendance, je reprendrai ma femme. Si cela ne marche pas, je reviendrai te voir à Alger. »

\section{CAS No 2 \\ Pulsions homicides indifférenciées chez un rescapé d'une liquidation collective}

S.... 37 ans, fellah. Habite un douar dans le Constantinois. Ne s'est jamais occupé de politique. Depuis le début de la guerre, sa région est le lieu de batailles violentes entre les forces algériennes et l'armée française. S... a ainsi l'occasion de voir des morts et des blessés. Mais il continue à se tenir à l'écart. De temps à autre, comme l'ensemble du peuple, les paysans de son village viennent en aide aux combattants algériens de passage.

[249]

Mais un jour, au début de 1958, a lieu une embuscade meurtrière non loin du douar. Les forces ennemies montent une opération et assiègent le village, d'ailleurs vide de soldats. Tous les habitants sont réunis et interrogés. Personne ne répond. Quelques heures après, un officier français arrive en hélicoptère et dit : «Ce douar fait trop parler de lui ; détruisez-le! » Les soldats commencent à mettre le feu aux maisons tandis que les femmes qui tentent de ramasser quelques vêtements ou de sauver quelques réserves sont refoulées à coups de crosse. Certains paysans profitent de la confusion qui règne pour s'enfuir. L'officier donne l'ordre de rassembler les hommes restants et les fait conduire près d'un oued où le massacre commence. Vingt-neuf hommes sont tués à bout portant. S... est blessé de deux balles qui lui traversent respectivement la cuisse droite et le bras gauche, cette dernière blessure lui occasionnant une fracture de l'humérus.

S... s'évanouit et reprend connaissance au milieu d'un groupe de I'ALN. Il est soigné par le service sanitaire et évacué quand il lui devient possible de se déplacer. En cours de route, son comportement de 
plus en plus anormal ne cesse d'inquiéter l'escorte. Il réclame un fusil, alors qu'il est civil et impotent, et refuse de marcher devant qui que ce soit. Il ne veut personne derrière lui. Une nuit, il s'empare de l'arme d'un combattant et maladroitement tire sur les soldats endormis. Est désarmé assez brutalement. Désormais il aura les mains liées, et c'est ainsi qu'il arrive au Centre.

Il commence par nous dire qu'il n'est pas mort et qu'il a joué un bon tour aux autres. Petit à petit, nous arrivons à reconstituer l'histoire de son assassinat manqué. S... n'est pas anxieux, mais plutôt surexcité, avec des phases d'agitation violente, accompagnées de hurlements. Il casse peu, mais fatigue tout le monde par son incessant bavardage, et le Service est en alerte permanente à cause de sa volonté affirmée de «tuer tout le monde». Au cours de son hospitalisation, il va s'attaquer, avec des armes de fortune, à près de huit malades. Les infirmiers et les médecins ne sont pas épargnés. On arrive même à se demander si l'on [250] ne se trouve pas en présence d'une de ces formes larvées d'épilepsie caractérisée par une agressivité globale presque toujours en érection.

Une cure de sommeil est entreprise. À partir du troisième jour, un entretien quotidien va nous permettre de mieux comprendre la dynamique du processus pathologique. Le désordre intellectuel s'estompe progressivement. Voici quelques passages des déclarations du malade:

«Dieu est avec moi... mais alors, il n'est pas avec ceux qui sont morts... J'ai eu une sacrée chance... Dans la vie, il faut tuer pour ne pas être tué... Quand je pense que je ne connaissais rien de leurs histoires... Il y a des Français parmi nous. Ils se déguisent en Arabes. Il faut tous les tuer. Donne-moi une mitraillette. Tous ces soi-disant Algériens sont des Français... et ils ne me laissent pas tranquille. Dès que je veux m'endormir, ils entrent dans ma chambre. Mais maintenant, je les connais. Tout le monde veut me tuer. Mais je me défendrai. Je les tuerai tous sans exception. Je les égorgerai les uns après les autres, et toi aussi avec. Vous voulez me descendre, mais il faudra vous y prendre autrement. Cela ne me fera rien de vous abattre. Les petits, 
les grands, les femmes, les enfants, les chiens, les oiseaux, les ânes ... tout le monde y passera... Après, je pourrai dormir tranquille ... »

Tout cela est dit dans un langage haché, l'attitude demeurant hostile, hautaine, méprisante.

Après trois semaines, l'excitation disparaît, mais une réticence, une certaine tendance à la solitude nous font craindre une évolution plus grave. Cependant, après un mois, il demande à sortir pour apprendre un métier compatible avec son infirmité. Il est alors confié au Service social du FLN. Revu six mois après. Va bien.

\section{CAS No 3 \\ Psychose anxieuse grave à type de dépersonnalisation après le meurtre forcené d'une femme}

Dj..., ancien étudiant, militaire dans l'ALN, 19 ans. Quand il arrive au Centre, sa maladie remonte à plusieurs mois. Sa présentation est caractéristique: fortement déprimé, les lèvres [251] sèches, les mains constamment moites. D'incessants soupirs soulèvent sa poitrine. Insomnie tenace. Deux tentatives de suicide depuis le début des troubles. Pendant la conversation, adopte des attitudes d'écoute hallucinatoire. Parfois le regard se fixe pendant quelques instants sur un point de l'espace tandis que le visage s'anime, donnant l'impression à l'observateur que le malade assiste à un spectacle. Pensées floues. Quelques phénomènes connus en psychiatrie sous le nom de barrage: un geste ou une phrase ébauchés sont brusquement interrompus sans raison apparente. Mais surtout, un élément va particulièrement retenir notre attention: le malade nous parle de son sang répandu, de ses artères qui se vident, de son cœur qui a des ratés. Il nous supplie d'arrêter l'hémorragie, de ne plus tolérer qu'on vienne le « vampiriser» jusqu'à l'hôpital. De temps à autre, n'arrive plus à parler et demande un crayon. Écrit : « Je n'ai plus de voix, toute ma vie s'en va. » Cette dépersonnalisation vécue nous fait penser à une évolution très grave. 
Plusieurs fois au cours de nos conversations, le malade nous parle d'une femme qui, la nuit venue, vient le persécuter. Ayant appris auparavant que sa mère est morte, qu'il l'aimait beaucoup, que rien ne saurait le consoler de cette perte (la voix s'est considérablement assourdie à ce moment et quelques larmes sont apparues), je dirige l'investigation sur l'image maternelle Comme je lui demande de décrire cette femme obsédante, persécutrice même, il me déclare que ce n'est pas une inconnue, qu'il la connaît très bien puisque c'est lui qui l'a tuée. La question se pose alors de savoir si nous sommes en présence d'un complexe de culpabilité inconscient après la mort de la mère comme Freud en a décrit dans Deuil et mélancolie. Nous demandons au malade, puisqu'il connaît si bien cette femme, puisque aussi bien c'est lui qui l'aurait tuée, de nous parler plus longuement. C'est ainsi que nous reconstituons l'histoire suivante.

« De la ville où j'étais étudiant, je suis monté au maquis. Après plusieurs mois, j'ai eu des nouvelles de chez moi. J'ai appris que ma mère avait été tuée à bout portant par un soldat [252] français et deux de mes sœurs emmenées chez les militaires. Jusqu'à présent, j'ignore ce qu'elles sont devenues. J'ai été terriblement ébranlé par la mort de ma mère. Mon père étant mort depuis plusieurs années, j'étais le seul homme de la famille, et ma seule ambition a toujours été d'arriver à quelque chose pour améliorer l'existence de ma mère et de mes scurs. Un jour, nous sommes allés dans une propriété de colons où le gérant, actif colonialiste, avait déjà abattu deux civils algériens. Nous sommes arrivés chez lui dans la nuit. Mais il n'était pas là. Il n'y avait dans la maison que sa femme. En nous voyant, elle se mit à nous supplier de ne pas la tuer: "Je sais que vous venez pour mon mari, dit-elle, mais il n'est pas là... combien de fois lui ai-je dit de ne pas se mêler de politique." On décida d'attendre le mari. Mais moi, je regardais la femme et je pensais à ma mère. Elle était assise dans un fauteuil et semblait absente. Je me demandais pourquoi on ne la tuait pas. Et à un moment, elle s'aperçut que je la regardais. Et elle se jeta sur moi en criant: "Je vous en prie... ne me tuez pas... J'ai des enfants." L'instant d'après elle 
était morte. Je l'avais tuée avec mon couteau. Le chef me désarma et donna l'ordre de partir. J'ai été interrogé par le chef de secteur quelques jours plus tard. Je croyais que j'allais être tué, mais je m'en fichais ${ }^{22}$. Et puis, je commençai à vomir après les repas, et à mal dormir. Ensuite, cette femme est venue chaque soir me réclamer mon sang. Et le sang de ma mère où est-il ? »

Le soir venu, dès que le malade se couche, la chambre « est envahie de femmes », toutes les mêmes. C'est une réédition en multiples exemplaires d'une seule femme. Elles portent toutes un trou béant dans le ventre. Elles sont exsangues, pâles et affreusement maigres. Ces femmes harcèlent le jeune malade et exigent qu'il leur rende leur sang répandu. À ce moment, un bruit d'eau qui coule emplit la chambre, s'amplifie jusqu'à [253] évoquer le tonnerre d'une cascade, et le jeune malade voit le parquet de la chambre s'imbiber de sang, son sang, tandis que les femmes deviennent de plus en plus roses, et que leur plaie commence à se fermer. Baigné de sueur et terriblement angoissé, le malade se réveille et demeure agité jusqu'à l'aube.

Le jeune malade est soigné plusieurs semaines et les phénomènes oniroïdes (cauchemars) ont pratiquement disparu. Cependant, une grande faille se maintient dans sa personnalité. Dès qu'il pense à sa mère, en double ahurissant surgit cette femme éventrée. Aussi peu scientifique que cela puisse sembler, nous pensons que seul le temps pourra apporter quelque amélioration dans la personnalité disloquée du jeune homme.

22 Après l'expertise médico-légale qui mit en évidence le caractère pathologique de l'acte, les poursuites judiciaires décidées par l'État-Major de l'ALN cessèrent. 


\section{CAS No 4 \\ Un gardien de police européen déprimé rencontre en milieu hospitalier une de ses victimes, un patriote algérien atteint de stupeur}

A.... 28 ans, marié, sans enfant. Nous apprenons que depuis plusieurs années, ils se soignent, sa femme et lui, malheureusement sans succès, pour avoir des enfants. Il nous est adressé par ses supérieurs pour troubles de comportement.

Le contact immédiat se révèle assez bon. Spontanément, le malade nous parle de ses difficultés; entente satisfaisante avec sa femme et avec ses beaux-parents. Bons rapports avec ses camarades de travail : ayant par ailleurs l'estime de ses supérieurs. Ce qui l'embête, c'est que la nuit il entend des cris qui l'empêchent de dormir. Et de fait, il nous apprend que, depuis plusieurs semaines, avant de se coucher il ferme les volets et calfeutre les fenêtres (nous sommes en été), au grand désespoir de sa femme qui étouffe de chaleur. De plus, il remplit ses oreilles de coton, afin d'atténuer la violence des cris. Quelquefois même, en pleine nuit, il allume le poste TSF ou met de la musique pour ne pas entendre ces nocturnes clameurs. Dès lors, A... va nous exposer très longuement son drame:

Depuis plusieurs mois, il est affecté à une brigade anti-FLN. Au début, il était chargé de la surveillance de quelques établis [254] sements ou cafés. Mais après quelques semaines, il travaillera presque constamment au commissariat. C'est alors qu'il a l'occasion de pratiquer des interrogatoires, ce qui ne va jamais sans «bousculades». "C'est qu'ils ne veulent rien avouer. »

«Des fois, explique-t-il, on a envie de leur dire que s'ils avaient un peu pitié pour nous, ils parleraient sans nous obliger à passer des heures pour leur arracher mot par mot les renseignements. Mais allez leur 
expliquer quelque chose. À toutes les questions posées, ils répondent "Je ne sais pas". Même leurs noms. Si on leur demande où ils habitent, ils disent "Je ne sais pas". Alors bien sûr... on est obligé d'y aller. Mais ils gueulent trop. Au début, ça me faisait rigoler. Mais après, cela commença à me secouer. Aujourd'hui, rien qu'en entendant quelqu'un crier, je peux vous dire où il en est, à quel stade on en est de l'interrogatoire. Le gars qui a reçu deux coups de poing et un coup de matraque derrière l'oreille a une certaine façon de parler, de crier, de dire qu'il est innocent. Après être resté deux heures suspendu par les poignets il a une autre voix. Après la baignoire, une autre voix. Et ainsi de suite. Mais c'est surtout après l'électricité que cela devient insupportable. On dirait à tout instant que le type va mourir. Il y a évidemment ceux qui ne crient pas: ce sont les durs. Mais ils s'imaginent qu'on va les tuer tout de suite. Nous, cela ne nous intéresse pas de les tuer. Ce qu'il nous faut, c'est le renseignement. Ceux-là, on cherche d'abord à les faire crier, et tôt ou tard ils y arrivent. Ça, c'est déjà une victoire. Après on continue. Remarquez qu'on aimerait bien éviter cela. Mais ils ne nous rendent pas la tâche facile. Maintenant j'en arrive à entendre ces cris même chez moi. Surtout les cris de quelquesuns qui sont morts au commissariat. Docteur, je suis dégoûté de ce boulot. Et si vous me guérissez, je demanderai ma mutation en France. S'ils refusent, je démissionnerai. »

Devant ce tableau je prescris un congé de maladie. Comme l'intéressé refuse l'hospitalisation, je le soigne à titre privé. Un jour, peu avant l'heure de la séance thérapeutique, je suis appelé [255] d'urgence dans mon service. À mon arrivée chez moi, ma femme invite A... à m'attendre, mais il préfère aller faire un tour dans l'hôpital et partir ainsi à ma rencontre. Quelques minutes plus tard, rentrant chez moi, je le trouve sur le chemin. Il est appuyé à un arbre, l'air manifestement accablé, tremblant, baigné de sueur, en pleine crise anxieuse. Je le prends dans ma voiture et le conduis chez moi. Une fois installé sur le divan, il me raconte avoir rencontré dans l'établissement un de mes malades qui avait été interrogé dans les locaux de la police (c'est 
un patriote algérien), et qui est soigné pour «troubles postcommotionnels de type stuporeux». J'apprends alors que ce policier a participé d'une manière effective aux tortures infligées à ce malade. J'administre quelques sédatifs qui calment l'anxiété de A... Après son départ, je me rends au pavillon où est hospitalisé le patriote. Le personnel ne s'est aperçu de rien. Le malade cependant demeure introuvable. Enfin, on arrive à le découvrir dans un lavabo où il tentait de se suicider (le malade avait de son côté reconnu le policier et croyait que celui-ci était venu le chercher pour le conduire à nouveau dans les locaux de la police).

Par la suite, A... est revenu me voir plusieurs fois, et, après une amélioration très nette, parvint pour raisons de santé à se faire rapatrier. Quant au patriote algérien, le personnel s'acharna longtemps à le persuader qu'il s'agissait d'une illusion, que les policiers ne pouvaient venir à l'hôpital, qu'il était fatigué, qu'il était ici pour être soigné, etc.

\section{CAS No 5 \\ Un inspecteur européen torture sa femme et ses enfants}

R.... 30 ans, vient spontanément nous consulter. Il est inspecteur de police, et constate depuis quelques semaines que «cela ne tourne pas rond ». Marié, trois enfants. Il fume beaucoup : cinq paquets de cigarettes par jour. Il n'a plus d'appétit et son sommeil est fréquemment agité de cauchemars. Ces cauchemars n'ont pas de caractéristiques propres. Ce qui le gêne le plus, c'est ce qu'il appelle ses « crises de folie». D'abord il [256] n'aime pas être contrarié : « Docteur, expliquezmoi cela. Dès que je rencontre une opposition j'éprouve l'envie de frapper. Même en dehors du boulot, j'ai envie de travailler le gars qui me barre la route. Un rien. Tenez, par exemple, je vais chercher les journaux au kiosque. Il y a beaucoup de monde. Forcément il faut attendre. Je tends le bras (le type qui tient le kiosque est un copain) pour prendre mes journaux. Quelqu'un dans la queue me dit avec un petit air de 
défi : "Attendez votre tour." Eh bien, j'ai envie de le taper, et je me dis "Mon petit vieux, si je te tenais quelques heures, tu ferais moins le zigomar après !'Il n'aime pas le bruit. À la maison, il a envie de frapper tout le monde, tout le temps. Et, effectivement, il frappe ses enfants, même le petit de 20 mois, avec une rare sauvagerie.

Mais ce qui l'a effrayé, c'est qu'un soir où sa femme l'avait particulièrement critiqué de trop frapper les enfants (elle lui avait même dit : «Ma parole, tu deviens fou... ») il s'est jeté sur elle, l'a battue et ligotée sur une chaise en lui disant: "Je vais t'apprendre une fois pour toutes que c'est moi le maître dans cette baraque. »

Heureusement, ses enfants commencent à pleurer et à crier. Il réalise alors la gravité de son comportement, détache sa femme et, le lendemain, décide de consulter un médecin «spécialisé dans les nerfs». Il précise qu'« avant [il] n'étai[t] pas comme cela», qu'il corrigeait rarement ses enfants et ne se disputait en tout cas jamais avec sa femme. Les phénomènes actuels sont apparus depuis «les événements»: «C'est que, dit-il, nous faisons maintenant un travail d'infanterie. La semaine dernière par exemple, nous étions en opération comme si nous appartenions à l'armée. Ces Messieurs du gouvernement disent qu'il n'y a pas de guerre en Algérie et que les forces de l'ordre, c'est-à-dire la police, doivent ramener le calme. Mais il y a la guerre en Algérie, et quand ils s'en rendront compte, ce sera trop tard. Ce qui me tue le plus ce sont les tortures. Ça ne vous dit rien, vous ?... Je torture des fois dix heures d'affilée...

- Qu'est-ce que cela vous fait de torturer?

[257]

- Mais cela fatigue... Il est vrai qu'on se relaie, mais c'est une question que de savoir à quel moment passer la main au copain. Chacun pense qu'il est sur le point d'obtenir le renseignement et se garde bien de céder l'oiseau préparé à l'autre, qui, naturellement, en tirera une gloire. Alors, on lâche... ou on ne lâche pas...

«Il arrive même qu'on propose au type de l'argent, notre propre argent de poche pour l'amener à parler. Le problème pour nous, en ef- 
fet, est le suivant : es-tu capable de faire parler ce type? C'est un problème de succès personnel ; on est en compétition, quoi... On a eu à la fin les poings esquintés. Alors, on a employé les "Sénégalais". Mais ils frappent soit trop fort et esquintent le type en une demi-heure, soit trop doucement et ce n'est pas efficace. En fait, il faut être intelligent pour réussir dans ce travail. Il faut savoir à quel moment serrer et à quel autre desserrer. C'est une question de flair. Quand le gars est mûr, ce n'est pas la peine de continuer à taper. C'est pourquoi il faut faire le travail soi-même: on surveille mieux les progrès. Je suis contre ceux qui font préparer le gars par d'autres et qui viennent voir toutes les heures où il en est. Ce qu'il faut surtout, c'est ne pas donner au type l'impression qu'il ne sortira pas vivant de vos mains. Il se demanderait alors pourquoi parler si cela ne doit pas lui sauver la vie. Dans ce cas-là vous n'auriez aucune chance de savoir quelque chose. Il faut qu'il espère : c'est l'espoir qui fait parler.

«Mais ce qui m'embête le plus, c'est l'histoire de ma femme. C'est sûr qu'il doit y avoir quelque chose de détraqué. Il faut marranger cela, Docteur. »

Son administration lui refusant un repos et, par ailleurs, le malade ne voulant pas de certificat d'un psychiatre, un traitement est entrepris en «pleine activité ». On devine aisément les faiblesses d'une pareille formule. Cet homme savait parfaitement que tous ses troubles étaient causés directement par le type d'activité déployée dans les salles d'interrogatoire, encore qu'il ait tenté d'en rejeter globalement la responsabilité sur " les événements». Comme il n'envisageait pas (ce serait un non-sens) [258] d'arrêter de torturer (alors il faudrait démissionner), il me demandait sans ambages de l'aider à torturer les patriotes algériens sans remords de conscience, sans troubles de comportement, avec sérénité 23.

23 On se trouve en présence avec cette observation d'un système cohérent qui ne laisse rien intact. Le bourreau qui aime les oiseaux ou jouit dans le calme d'une symphonie ou d'une sonate, c'est tout simplement une étape. Plus loin, il y a bel et bien une existence qui s'inscrit sur le registre d'un sadisme radical et absolu. 
[259]

\section{LES DAMNÉS DE LA TERRE}

\section{$\mathbf{V}$}

Série B

Retour à la table des matières

Nous avons rassemblé ici quelques cas ou groupes de cas où l'événement déclenchant est d'abord l'atmosphère de guerre totale qui règne en Algérie.

\section{CAS No 1 \\ Assassinat par deux jeunes algériens de 13 et 14 ans de leur camarade de jeux européen}

Il s'agit d'une expertise médico-légale. Deux jeunes Algériens de 13 et 14 ans, élèves d'une école primaire, sont accusés d'avoir tué un de leurs camarades européens. Ils ont reconnu avoir commis l'acte. Le crime est reconstitué, et des photos sont jointes au dossier. On y voit l'un des enfants tenir la victime tandis que l'autre frappe d'un couteau. Les petits inculpés ne reviennent pas sur leurs déclarations. Nous avons de longs entretiens avec eux. Nous reproduisons ici leurs propos caractéristiques. 
a) Celui de 13 ans.

« Nous n'étions pas fâchés avec lui. Tous les jeudis on allait chasser ensemble à la fronde, sur la colline, au-dessus du village. C'était notre bon copain. Il n'allait plus à l'école, car il voulait devenir maçon comme son père. Un jour on a décidé de le tuer, parce que les Européens ils veulent tuer tous les Algériens. Nous, on ne peut pas tuer les "grands". Mais comme lui, il a notre âge, on peut. On ne savait pas comment le tuer. On voulait le jeter dans un fossé, mais il aurait pu être seulement blessé. Alors, on a pris un couteau à la maison et on l'a tué.

[260]

- Mais pourquoi l'avoir choisi, lui ?

- Parce qu'il jouait avec nous. Un autre ne serait pas monté avec nous là-haut.

- Pourtant c'était un copain?

- Aussi, pourquoi ils veulent nous tuer? Son père, il est milicien, et il dit qu'il faut nous égorger

- Mais lui ne t'avait rien dit?

- Lui ? Non.

- Tu sais qu'il est mort maintenant?

- Oui.

- Qu'est-ce que la mort?

- C'est quand c'est fini, on va au ciel.

- C'est toi qui l'as tué?

- Oui.

- Est-ce que cela te fait quelque chose d'avoir tué quelqu'un?

- Non, puisqu'ils veulent nous tuer, alors...

- Cela t'embête-t-il d'être en prison? 
- Non. »

\section{b) Celui de 14 ans.}

Ce jeune inculpé contraste nettement avec son camarade. C'est déjà presque un homme, un adulte par le contrôle musculaire, la physionomie, le ton et le contenu des réponses. Lui non plus ne nie pas avoir tué. Pourquoi a-t-il tué ? Il ne répond pas à la question, mais me demande si j'ai déjà vu un Européen en prison. Y a-t-il jamais eu en prison un Européen arrêté après le meurtre d'un Algérien? Je lui réponds qu'effectivement je n'ai pas vu d'Européens en prison.

- Et pourtant, il y a des Algériens qui sont tués tous les jours, non

- oui.

- Alors, pourquoi ne trouve-t-on que les Algériens dans les prisons? Vous pouvez m'expliquer?

- Non, mais dis-moi pourquoi tu as tué ce garçon qui était ton copain?

[261]

- Je vais vous expliquer... Vous avez entendu parler de l'affaire de Rivet 24 ?

- Oui.

- Deux de mes parents ont été tués ce jour-là. Chez nous, on a dit que les Français avaient juré de nous tuer tous les uns après les autres. Est-ce qu'on a arrêté un Français pour tous ces Algériens qui ont été tués?

- Je ne sais pas.

24 Rivet est un village qui, depuis certain jour de l'année 1956, est devenu célèbre dans la région de l'Algérois. Un soir, en effet, le village fut envahi par des miliciens français qui, après avoir arraché de leurs lits quarante hommes, les assassinèrent. 
- Eh bien, personne n'a été arrêté. Moi, je voulais monter au djebel, mais je suis trop petit. Alors on a dit avec X... qu'il fallait tuer un Européen.

- Pourquoi?

- Qu'est-ce qu'il fallait faire d'après vous?

- Je ne sais pas. Mais tu es un enfant et ce sont des choses de grandes personnes qui se passent.

- Mais ils tuent aussi les enfants...

- Mais ce n'était pas une raison pour tuer ton copain.

- Eh bien, je l'ai tué. Maintenant, faites ce que vous voulez.

- Est-ce que ce copain t'avait fait quelque chose?

- Non, il ne mavait rien fait.

- Alors ?...

- Voilà...

\section{CAS No 2 \\ Délire d'accusation et conduite-suicide déguisée en « acte terroriste» chez un jeune algérien de 22 ans}

Ce malade est adressé à l'hôpital par l'autorité judiciaire française. Cette mesure est intervenue après une expertise médico-légale pratiquée par des psychiatres français exerçant en Algérie.

[262]

Il s'agit d'un homme amaigri, en plein état confusionnel. Le corps est couvert d'ecchymoses et deux fractures de la mâchoire rendent toute absorption d'aliments impossible. Aussi, pendant plus de deux semaines, nourrira-t-on le malade à l'aide d'injections diverses. 
Au bout de deux semaines, le vide de la pensée s'estompe: un contact peut être établi et nous arrivons à reconstituer l'histoire dramatique de ce jeune homme.

Pendant sa jeunesse, a pratiqué le scoutisme avec une rare ferveur. Est devenu l'un des principaux responsables du mouvement scout musulman. Mais à 19 ans, il négligea totalement le scoutisme pour ne plus se préoccuper que de sa profession. Mécanographe, il étudie avec ténacité et rêve de devenir un grand spécialiste dans son métier. Le ler novembre 1954 le trouvera absorbé par des problèmes strictement professionnels. N'a sur le moment aucune réaction à l'égard de la lutte nationale. Déjà il ne fréquentait plus ses anciens camarades. Il se définira lui-même à cette époque comme «mobilisé pour approfondir ses capacités techniques».

Pourtant, vers le milieu de 1955, au cours d'une veillée familiale, a soudain l'impression que ses parents le considèrent comme un traître. Après quelques jours, cette impression fugitive s'émousse mais il reste chez lui une certaine inquiétude, un certain malaise qu'il ne parvient pas à comprendre.

Il décide donc de prendre ses repas en vitesse, fuit le milieu familial et s'enferme dans sa chambre. Évite tous les contacts. C'est dans ces conditions que survient la catastrophe. Un jour, en pleine rue, vers midi et demi, il entend distinctement une voix le traiter de lâche. Il se retourne, mais ne voit personne. Il presse le pas et décide de ne plus aller travailler. Il reste dans sa chambre et ne dîne pas. Dans la nuit éclate la crise. Pendant trois heures, il entend toutes sortes d'insultes, des voix dans sa tête et dans la nuit : « Traître... lâche... tous tes frères qui meurent... traître... traître... »

Une anxiété indescriptible s'empare de lui : «Mon cœur a battu pendant 18 heures à la cadence de 130 à la minute. Je croyais que j'allais mourir. »

[263]

Dès lors, le malade ne peut plus rien avaler. Il maigrit à vue d'œil, se confine dans une obscurité absolue, refuse d'ouvrir à ses parents. 
Vers le troisième jour, il se jette dans la prière. Il gardera, me dit-il, la position agenouillée de 17 à 18 heures par jour. Le quatrième jour, impulsivement, "comme un fou», avec " une barbe qui devait aussi le faire prendre pour un fou $»$, sans veste et sans cravate, il sort dans la ville. Une fois dans la rue, il ne sait où aller; mais il marche et se retrouve au bout d'un certain temps dans la ville européenne. Son type physique (il ressemble à un Européen) semble alors le protéger des interpellations et des contrôles des patrouilles françaises.

Par contre, à côté de lui, des Algériens et des Algériennes sont arrêtés, bousculés, insultés, fouillés... Or, paradoxalement, il n'a aucun papier. Cette gentillesse spontanée des patrouilles ennemies à son égard le confirme dans son délire: "Tout le monde sait qu'il est avec les Français. Les soldats eux-mêmes ont des consignes: ils le laissent tranquille. »

De plus, le regard des Algériens arrêtés, les mains derrière la nuque, attendant la fouille, lui semble chargé de mépris. En proie à une agitation incoercible, il s'éloigne à grands pas. C'est à ce moment qu'il parvient devant l'immeuble de l'état-major français. À la grille, plusieurs militaires, mitraillette au point. Il s'avance vers les soldats, se jette sur l'un d'eux et essaie de lui arracher sa mitraillette en criant: "Je suis un Algérien. »

Rapidement maîtrisé, il est conduit dans les locaux de la police où l'on s'obstine à lui faire avouer les noms de ses chefs et ceux des différents membres du réseau auquel il appartient. Au bout de quelques jours les policiers et les militaires s'aperçoivent qu'ils ont affaire à un malade. Une expertise est décidée, qui conclut à l'existence de troubles mentaux et prescrit l'hospitalisation. « Ce que je voulais, nous ditil, c'était mourir. Même à la police, je croyais et j'espérais qu'après les tortures ils me tueraient. J'étais content d'être frappé, car cela me prouvait qu'ils me considéraient moi aussi comme leur ennemi. Je ne 
pouvais plus entendre sans réagir ces accusations. Je ne suis [264] pas un lâche. Je ne suis pas une femme. Je ne suis pas un traître ${ }^{25}$. »

\section{CAS No 3 \\ Attitude névrotique chez une jeune française dont le père, haut fonctionnaire, est tué dans une embuscade}

Cette jeune fille de 21 ans, étudiante, me consulte pour de petits phénomènes de type anxieux qui la gênent dans ses études et dans ses relations sociales. Paumes constamment moites, avec des périodes véritablement inquiétantes où l'eau « lui coule des mains». Oppressions thoraciques accompagnées de migraines nocturnes. Se ronge les ongles. Mais ce qui retient l'attention, c'est surtout la facilité du contact manifestement trop rapide, alors que se sent, sous-jacente, une angoisse importante. La mort de son père, récente pourtant d'après la date, est signalée par la malade avec une telle légèreté que nous orientons rapidement nos investigations sur ses rapports avec son père. L'exposé qui nous est fait, clair, absolument lucide, d'une lucidité qui frise l'insensibilité, va révéler, précisément par son rationalisme, le trouble de cette jeune fille, la nature et l'origine de son conflit.

«Mon père était un haut fonctionnaire. Il avait sous sa responsabilité une immense région rurale. Dès les événements, il s'est jeté dans la chasse aux Algériens avec une rage forcenée. Il arrivait à ne plus manger du tout, à ne plus dormir tellement ça l'excitait de réprimer la rébellion. J'ai assisté sans rien pouvoir faire à la lente métamorphose de mon père. À la fin, je décidai de ne plus aller le voir, de rester en ville. En effet, chaque fois que je me trouvais à la maison, je restais

25 Au cours de l'année 1955, les cas de ce genre furent extrêmement nombreux en Algérie. Malheureusement tous les malades n'eurent pas la chance d'arriver à I'hôpital. 
des nuits éveillée, car, venant d'en bas jusqu'à moi, les cris ne cessaient de me gêner: dans la cave et dans les pièces désaffectées, on torturait [265] des Algériens afin d'obtenir des renseignements. Vous ne pouvez vous imaginer ce que cela peut être affreux d'entendre crier ainsi toute la nuit. Des fois, je me demande comment un être humain peut supporter - je ne parle pas de torturer - mais simplement d'entendre crier de souffrance. Et cela durait. À la fin, je ne suis pas revenue à la maison. Les rares fois où mon père venait me voir en ville je n'arrivais pas à le regarder en face sans être horriblement gênée et effrayée. Cela me devenait de plus en plus difficile de l'embrasser.

«C'est que j'ai habité longtemps au village. J'en connais presque toutes les familles. Les jeunes Algériens de mon âge et moi avons joué ensemble quand nous étions petits. Chaque fois que je venais à la maison mon père m'apprenait que de nouvelles personnes avaient été arrêtées. À la fin, je n'osais plus marcher dans la rue tellement j'étais sûre de rencontrer partout la haine. Au fond de moi-même, je leur donnais raison à ces Algériens. Si j'étais algérienne, je serais au maquis. »

Un jour, cependant, elle reçoit un télégramme qui lui apprend que son père est grièvement blessé. Elle se rend à l'hôpital et trouve son père dans le coma. Il mourra peu après. C'est au cours d'une mission de reconnaissance avec un détachement militaire que son père a été blessé : la patrouille est tombée dans une embuscade tendue par l'Armée nationale algérienne.

"L'enterrement m'a éccurée, dit-elle. Tous ces officiels qui venaient pleurer sur la mort de mon père dont "les hautes qualités morales avaient conquis la population indigène" me donnaient la nausée. Tout le monde savait que c'était faux. Personne n'ignorait que mon père avait la haute main sur les centres d'interrogatoire de toute la région. On savait que le nombre de tués sous la torture atteignait dix par jour, et l'on venait réciter des mensonges sur le dévouement, l'abnégation, l'amour de la patrie, etc. Je dois dire que, maintenant, les mots pour moi n'ont plus de valeur, pas beaucoup en tout cas. Je suis 
rentrée immédiatement à la ville et j'ai fui toutes les autorités. On m'a proposé des subventions mais j'ai refusé. Je ne veux pas de leur [266] argent. C'est le prix du sang versé par mon père. Je n'en veux pas. Je travaillerai. »

\section{CAS NO 4 \\ Troubles du comportement chez de jeunes algériens de moins de 10 ans}

Il s'agit de réfugiés. Ce sont des fils de combattants ou de civils tués par les Français. Ils sont répartis dans différents centres en Tunisie et au Maroc. Ces enfants sont scolarisés. Des séances de jeux, des sorties collectives sont organisées. Les enfants sont suivis régulièrement par des médecins. C'est ainsi que nous avons l'occasion d'en voir un certain nombre.

a) Il existe chez ces différents enfants un amour très marqué pour les images parentales. Tout ce qui ressemble à un père ou à une mère est recherché avec une grande ténacité et jalousement gardé.

b) On remarque chez eux, d'une manière générale, une phobie du bruit. Ces enfants sont très affectés dès qu'on les réprimande. Grande soif de calme et d'affection.

c) Chez beaucoup on trouve des insomnies avec somnambulisme.

d) Énurésie périodique.

e) Tendance sadique. Un jeu fréquent : une feuille de papier tendue est percée rageusement de multiples trous. Les crayons sont tous mordus, et les ongles rongés avec une constance désespérante. Des disputes sont fréquentes entre eux malgré un fond de grande affection. 


\section{CAS NO 5 \\ Psychoses puerpérales chez les réfugiées}

On appelle psychose puerpérale les troubles mentaux qui surviennent chez la femme à l'occasion de la maternité. Ces [267] troubles peuvent apparaître immédiatement avant ou quelques semaines après l'accouchement. Le déterminisme de ces maladies est très complexe. Mais on estime que les deux causes principales sont un bouleversement du fonctionnement des glandes endocrines et l'existence d'un « choc affectif ». Cette dernière rubrique, quoique vague, recouvre ce que le public appelle « grosse émotion».

Sur les frontières tunisiennes et marocaines, depuis la décision prise par le gouvernement français de pratiquer sur des centaines de kilomètres la politique du glacis et de la terre brûlée, se trouvent près de 300000 réfugiés. On sait l'état de dénuement dans lequel ils vivent. Des commissions de la Croix-Rouge internationale se sont à maintes reprises rendues sur les lieux et, après avoir constaté l'extrême misère et la précarité des conditions de vie, ont recommandé aux organismes internationaux d'intensifier l'aide à ces réfugiés. Il était donc prévisible, étant donné la sous-alimentation qui règne dans ces camps, que les femmes enceintes montrent une particulière prédisposition à l'éclosion de psychoses puerpérales.

Les fréquentes invasions des troupes françaises appliquant «le droit de suite et de poursuite », les raids aériens, les mitraillages - on sait que les bombardements des territoires marocains et tunisiens par l'armée française ne se comptent plus, et Sakiet-Sidi-Youssef, le village martyr de Tunisie, en est le plus sanglant exemple -, l'état de démembrement familial, conséquence des conditions de l'exode, entretiennent chez ces réfugiés une atmosphère d'insécurité permanente. Disons-le, il y a peu d'Algériennes réfugiées ayant accouché qui n'aient présenté des troubles mentaux. 
Ces troubles revêtent plusieurs formes. Ce sont soit des agitations qui peuvent prendre quelquefois l'allure de furies, soit de grosses dépressions immobiles avec tentatives multiples de suicide, soit enfin des états anxieux avec pleurs, lamentations, appels à la miséricorde, etc. Pareillement, le contenu délirant est divers. On trouve soit un délire de persécution vague, qui intéresse n'importe qui, soit une agressivité délirante contre les [268] Français qui veulent tuer l'enfant à naître ou nouvellement né, soit une impression de mort imminente, les malades implorent alors des bourreaux invisibles d'épargner leur enfant...

Ici encore il faut signaler que les contenus fondamentaux ne sont pas balayés par la sédation et la régression des troubles. La situation des malades guéries entretient et nourrit ces nœuds pathologiques. 


\title{
LES DAMNÉS DE LA TERRE
}

\author{
$\mathbf{V}$ \\ Série C
}

\section{Modifications affectivo-intellectuelles et troubles mentaux après la torture}

Retour à la table des matières

Nous grouperons dans cette série les malades plus ou moins graves dont les troubles sont apparus immédiatement après ou pendant les tortures. Nous décrirons des sous-groupes, car nous nous sommes rendu compte qu'à chaque méthode de torture correspondaient, indépendamment d'une atteinte grossière ou profonde de la personnalité, des types morbides caractéristiques.

\section{LOT $N^{0} 1$ \\ Après les tortures indifférenciées dites préventives}

Nous faisons allusion ici aux méthodes brutales où il s'agit moins de tortures que de faire parler. Le principe qui veut qu'au-delà d'un certain seuil la souffrance devienne intolérable prend ici une singulière importance. Le but est donc de parvenir le plus rapidement possible à 
ce seuil. Le fignolage n'est pas pratiqué. Il y a attaque massive et multiforme : plusieurs policiers frappant en même temps : quatre policiers debout, encerclent le prisonnier et jonglent avec lui à coups de poing, tandis qu'un policier lui brûle la poitrine avec une cigarette et qu'un autre lui frappe la plante des pieds à coups de bâton... Quelques-unes des méthodes de torture utilisées en Algérie nous ont paru particulièrement atroces, toujours en nous référant aux confidences des torturés.

[270]

a) Injection d'eau par la bouche accompagnée de lavement à forte pression d'eau savonneuse 26.

b) Introduction d'une bouteille dans l'anus.

Deux formes de supplice dit « de l'immobilité »:

c) Le prisonnier est placé à genoux, les bras parallèles au sol, les paumes tournées vers le ciel, le buste et la tête droits. Aucun mouvement n'est permis. Derrière le prisonnier, un policier assis sur une chaise le ramène à l'immobilité à coups de matraque.

d) Le prisonnier est debout, la face contre le mur, les bras levés et les mains collées au mur. Ici également, au moindre mouvement, à la moindre ébauche de relâchement, les coups pleuvent.

Précisons maintenant qu'il existe deux catégories de torturés :

1) Ceux qui savent quelque chose.

2) Ceux qui ne savent rien.

26 Ce type de torture est la cause d'un grand nombre de décès. Après ces lavements à haute pression, en effet, la muqueuse intestinale est le lieu de multiples lésions provoquant des microperforations intestinales. Les embolies gazeuses et les péritonites sont alors très fréquentes. 
1) Ceux qui savent quelque chose sont rarement vus dans les formations sanitaires. Certes, on n'ignore pas que tel patriote a été torturé dans les prisons françaises, mais on ne le rencontre pas en tant que malade 27.

2) Par contre, ceux qui ne savent rien, vont très fréquemment nous consulter. Nous ne parlons pas ici des Algériens frappés au cours d'un ratissage ou d'un bouclage. Ceux-là non plus ne viennent pas à nous en malades. Nous parlons expressément de ces Algériens, non organisés, arrêtés, conduits dans les locaux [271] de la police ou dans les fermes d'interrogatoires pour y être soumis à la question.

\section{TABLEAUX PSYCHIATRIQUES RENCONTRÉS}

a) Dépressions agitées : quatre cas

Ce sont des malades tristes, sans anxiété réelle, déprimés, confinés la plupart du temps au lit, qui fuient le contact, et qui, brusquement, vont développer une agitation très violente dont il est toujours difficile de comprendre la signification.

\section{b) Anorexie mentale : cinq cas}

Ces malades posent des problèmes graves, car cette anorexie mentale s'accompagne d'une phobie de tout contact corporel avec autrui. L'infirmier qui s'approche du malade et tente de le toucher, de lui prendre la main, par exemple, est immédiatement rejeté avec rigidité.

27 Nous parlons évidemment d'Algériens qui, sachant quelque chose, n'ont pas avoué sous la torture car on sait bien qu'un Algérien qui avoue est tué aussitôt après. 
Il n'est pas possible de pratiquer une alimentation artificielle ou d'administrer des médicaments 28.

\section{c) Instabilité motrice : onze cas}

Ici nous avons affaire à des malades qui ne restent pas en place. Continuellement solitaires, ils acceptent difficilement de s'enfermer avec le médecin dans son bureau.

Deux sentiments nous ont paru fréquents dans ce premier lot de torturés :

D'abord celui de l'injustice. Avoir été torturé pour rien, durant des jours et des nuits, semble avoir cassé quelque chose chez ces hommes. L'un de ces martyrisés avait eu une expérience particulièrement pénible: après plusieurs jours de vaines tortures, les policiers acquirent la conviction qu'ils avaient affaire à un homme paisible, totalement étranger à l'un [272] quelconque des réseaux FLN. En dépit de cette conviction, un inspecteur de police aurait dit : « Ne le lâchez pas comme cela. Serrez-le encore un peu. Ainsi quand il sera dehors, il restera tranquille 29 .»

Ensuite, une indifférence à tout argument moral. Pour ces malades, il n'y a pas de cause juste. Une cause torturée est une cause faible. Donc il faut s'occuper avant tout d'augmenter sa force, et ne pas se poser la question du bien-fondé d'une cause. Seule compte la force.

28 Le corps médical doit se relayer jour et nuit auprès du malade dans un travail d'explication. On comprend que la formule «brutalisons un peu le malade» ne puisse être valablement utilisée ici.

29 Cette torture préventive devient, dans certaines régions, « répression préventive». C'est ainsi qu'à Rivet, alors que le calme régnait, les colons ne voulant pas être pris au dépourvu (les régions avoisinantes commençaient à s'agiter) décidèrent de supprimer purement et simplement les éventuels membres du FLN. Plus de quarante Algériens furent tués en une seule journée. 


\section{LOT No 2 \\ Après les tortures à l'électricité}

Dans ce lot nous avons rangé les patriotes algériens torturés principalement à l'électricité. En effet, alors qu'auparavant l'électricité faisait partie d'un ensemble de procédés de torture, à partir de septembre 1956, certains interrogatoires auront lieu exclusivement à l'électricité.

\section{TABLEAUX PSYCHIATRIQUES RENCONTRÉS}

a) Cénesthopathies localisées ou généralisées: trois cas

Il s'agit de malades ressentant des fourmillements dans le corps, impression de main qu'on arrache, de tête qui éclate, de langue qu'on avale

b) Apathie, aboulie, désintérêt : sept cas

Ce sont des malades inertes, sans projet, sans ressort, qui vivent au jour le jour.

c) Peur phobique de l'électricité

Peur de côtoyer un interrupteur, peur d'ouvrir la radio, peur du téléphone. Impossibilité absolue pour le médecin d'évoquer seulement l'éventualité d'un traitement par choc électrique. 


\section{LOT No 3 \\ Après le « sérum de vérité »}

On connaît le principe de ce traitement. Devant un malade qui semble souffrir d'un conflit intérieur inconscient que l'entretien n'arrive pas à extérioriser, on recourt à des méthodes d'exploration chimique. Le penthotal, par injections intraveineuses, est la substance la plus communément pratiquée dans le but de libérer le malade d'un conflit qui paraît dépasser ses possibilités d'adaptation. C'est pour libérer le malade de ce «corps étranger » que le médecin intervient ${ }^{30}$. Toutefois, on s'est aperçu de la difficulté qu'il y avait à contrôler la dissolution progressive des instances psychiques. Il n'était pas rare d'assister à des aggravations spectaculaires ou à l'apparition de nouveaux tableaux absolument inexplicables. Aussi, d'une façon générale, a-t-on plus ou moins abandonné cette technique.

En Algérie, les médecins militaires et les psychiatres ont trouvé dans les salles de police de grandes possibilités d'expérimentation. Si, dans les névroses, le penthotal balaie les barrages qui s'opposent à la mise au jour du conflit intérieur, chez les patriotes algériens il doit pouvoir également briser le barrage politique et faciliter l'obtention des aveux du prisonnier sans qu'on ait besoin de recourir à l'électricité (la tradition médicale veut qu'on épargne la souffrance). C'est la forme médicale de la « guerre subversive».

Le scénario est le suivant. D'abord: «Je suis médecin, je ne suis pas un policier. Je suis là pour t'aider. »Ce faisant, on [274] obtient au bout de quelques jours la confiance du prisonnier 31 . Ensuite: « Je

30 En fait, il n'est pas étranger du tout. Le conflit n'est que le résultat de l'évolution dynamique de la personnalité où il ne saurait y avoir de «corps étranger». Disons plutôt qu'il s'agit d'un corps mal intégré.

31 Nous citerons également le cas de psychiatres animant les groupes «Présence française » qui, désignés pour expertiser un prisonnier, avaient l'habitude, au premier contact, de proclamer leur grande amitié avec l'avocat défenseur et 
vais te faire quelques piqûres, car tu es drôlement sonné. » Pendant plusieurs jours, on met en train n'importe quel traitement : vitamines, tonicardiaques, sérums sucrés. Le quatrième ou le cinquième jour, injection intraveineuse de penthotal. L'interrogatoire commence.

\section{TABLEAUX PSYCHIATRIQUES RENCONTRÉS}

\section{a) Stéréotypies verbales}

Le malade répète continuellement des phrases du type: «Je n'ai rien dit. Il faut me croire, je n'ai pas parlé. " Ces stéréotypies s'accompagnent d'une angoisse permanente. Le malade en effet, très souvent, ignore si on a pu lui arracher des renseignements. La culpabilité envers la cause défendue et les frères dont on a pu donner les noms et les adresses pèse ici de façon dramatique. Nulle affirmation ne peut ramener le calme dans ces consciences délabrées.

\section{b) Perception intellectuelle ou sensorielle opacifiée}

Le malade ne peut pas affirmer l'existence de tel objet perçu. Un raisonnement est assimilé, mais de façon indifférenciée. Il y a une indistinction fondamentale du vrai et du faux. Tout est vrai et tout est faux à la fois.

\section{c) Crainte phobique de tout tête-à-tête}

Cette crainte dérive de l'impression aiguë qu'on peut à tout instant être interrogé de nouveau.

d'affirmer qu'à eux deux (l'avocat et lui) ils sortiraient de là le prisonnier. Tous les prisonniers expertisés dans ces conditions ont été guillotinés. Ces psychiatres se vantaient devant nous de cette manière élégante de vaincre les « résistances». 


\section{d) Inhibition}

Le malade se tient sur ses gardes: il enregistre mot après mot la question posée, élabore mot après mot la réponse projetée. D'où l'impression de quasi-inhibition, avec ralentissement psychique, interruption des phrases, retours en arrière, etc.

Il est clair que ces malades refusent obstinément toute injection intraveineuse.

\section{$\operatorname{LOT} N^{\circ} 4$ Après lavage de cerveau}

On a beaucoup parlé ces derniers temps de l'« action psychologique en Algérie. Nous ne voulons pas procéder à l'étude critique de ces méthodes. Nous nous contenterons d'évoquer ici leurs conséquences psychiatriques. Il existe deux catégories de centres de torture par lavage de cerveau en Algérie.

\section{I - POUR LES INTELLECTUELS}

Le principe est ici d'amener le prisonnier à jouer un rôle. On voit à quelle école psychosociologique cela renvoie ${ }^{32}$.

32 On sait qu'il s'est développé aux États-Unis d'Amérique un courant psychosociologique. Des tenants de cette école pensent que le drame de l'individu contemporain est contenu dans le fait qu'il ne tient plus de rôle, que le mécanisme social l'accule à n'être qu'un rouage. D'où la thérapeutique proposée pour permettre à I'homme de tenir des rôles dans une véritable activité ludique. On joue n'importe quel rôle, on change même de rôle dans la même journée, on est capable de se mettre à la place de n'importe qui symboliquement. Les psychiatres d'usine aux États-Unis font paraît-il des prodiges dans la psychothérapie de groupes des ou- 


\section{a) Mener le jeu de la collaboration}

L'intellectuel est invité à collaborer en élaborant des justifications à cette collaboration. Il est donc obligé de mener une existence dédoublée: c'est un patriote connu comme tel qui, [276] préventivement, a été retiré de la circulation. Le but de l'action entreprise est d'attaquer de l'intérieur les éléments qui constituent la conscience nationale. Non seulement il doit collaborer, mais la consigne lui est donnée de discuter «librement » avec les opposants ou les réticents et de les convaincre. C'est là une manière élégante de l'amener à attirer l'attention sur les patriotes, donc à servir d'indicateur. Si par hasard il affirme ne pas trouver d'opposants, on les lui désigne ou on lui demande de faire comme s'il s'agissait d'opposants.

b) Faire des exposés sur la valeur de l'ceuvre française et sur le bien-fondé de la colonisation

Pour mener à bien cette tâche, on est largement entouré de « conseillers politiques »: officiers des Affaires indigènes, ou mieux encore : psychologues, psychologues de la vie sociale, sociologues, etc.

c) Prendre les arguments de la Révolution algérienne et les combattre un à un

L'Algérie n'est pas une nation, n'a jamais été une nation, ne sera jamais une nation.

Il n'y a pas de « peuple algérien ».

Le patriotisme algérien est un non-sens.

Les « fellagas » sont des ambitieux, des criminels, de pauvres types trompés.

vriers. On leur permet en effet de s'identifier à des héros. La tension dans les rapports patrons-ouvriers s'en trouve considérablement diminuée. 
Tour à tour, chaque intellectuel doit faire un exposé sur ces thèmes, et l'exposé doit être convaincant. Des notes (les fameuses « récompenses ») sont attribuées et totalisées à la fin de chaque mois. Elles serviront d'éléments d'appréciation pour décider ou non de la sortie de l'intellectuel.

d) Mener une vie collective absolument pathologique

Être seul est un acte de rébellion. Aussi est-on toujours avec quelqu'un. Le silence également est prohibé. Il faut penser à voix haute [277]

TÉMOIGNAGE

Il s'agit d'un universitaire interné et soumis des mois durant au lavage de cerveau. Les responsables du camp, certain jour, le félicitent pour les progrès réalisés et lui annoncent sa libération prochaine.

Connaissant les mancuvres de l'ennemi, il se garde de prendre cette nouvelle au sérieux. La technique est, en effet, d'annoncer aux prisonniers leur sortie et quelques jours avant la date fixée d'organiser une séance de critique collective. À la fin de la séance la décision est alors souvent prise de surseoir à la libération, le prisonnier ne paraissant pas présenter tous les signes d'une guérison définitive. La séance, disent les psychologues présents, a mis en évidence la persistance du virus nationaliste.

Cette fois pourtant, il ne s'agit pas d'un subterfuge. Le prisonnier est bel et bien libéré. Une fois dehors, dans la ville et au sein de sa famille, l'ancien prisonnier se félicite d'avoir si bien joué son rôle. Il se réjouit de pouvoir reprendre sa place dans le combat national et tente déjà d'établir le contact avec ses responsables. C'est à ce moment qu'une idée lancinante et terrible lui traverse l'esprit. Peut-être n'a-til trompé personne, ni les geôliers, ni les codétenus, ni surtout luimême. 
Où devait finir le jeu?

Là encore, il faut rassurer, lever l'hypothèse de la culpabilité.

\section{TABLEAUX PSYCHIATRIQUES RENCONTRÉS}

a) Phobie de toute discussion collective. Dès qu'il y a rencontre à trois ou quatre, l'inhibition réapparaît, la méfiance, la réticence s'imposent avec une particulière densité.

b) Impossibilité d'expliquer et de défendre une position donnée. La pensée se déroule par couples antithétiques. Tout ce qui est affirmé peut, dans le même moment, être nié avec la même force. C'est certainement la séquelle la plus douloureuse que nous ayons rencontrée dans cette guerre. Une personnalité [278] obsessionnelle est le fruit de I'« action psychologique » mise au service du colonialisme en Algérie.

\section{II - POUR LES NON-INTELLECTUELS}

Dans les centres comme Berrouaghia, on ne part plus de la subjectivité pour modifier les attitudes de l'individu. On s'appuie, au contraire, sur le corps que l'on casse, en espérant que la conscience nationale se démantèlera. C'est un véritable dressage. La récompense se traduit par l'absence de tortures ou par la possibilité de s'alimenter.

a) Il faut avouer que l'on n'est pas FLN. Il faut le crier en groupe. Il faut le répéter des heures durant.

b) Ensuite, il faut reconnaître qu'on a été FLN et qu'on a compris que c'était mal. Donc: à bas le FLN.

Après cette étape, en arrive une autre: l'avenir de l'Algérie est français, il ne peut être que français. 
Sans la France, l'Algérie retourne au Moyen Âge.

Enfin, on est français. Vive la France.

Ici les troubles rencontrés ne sont pas graves. C'est le corps souffrant et douloureux qui appelle repos et apaisement. 
[279]

\title{
LES DAMNÉS DE LA TERRE
}

\author{
$\mathbf{V}$ \\ Série D
}

\section{Troubles psychosomatiques}

\section{Retour à la table des matières}

La guerre coloniale d'Algérie n'a pas seulement eu comme conséquence de multiplier les troubles mentaux et de favoriser l'éclosion de phénomènes morbides spécifiques. En dehors de la pathologie de la torture, de la pathologie du torturé et de celle du tortionnaire foisonne en Algérie une pathologie d'atmosphère, celle qui fait dire communément aux médecins-praticiens en présence d'un malade qu'ils n'arrivent pas à comprendre: «Tout cela finira avec, cette sacrée guerre. »

Nous proposons de ranger dans cette quatrième série les maladies rencontrées chez les Algériens dont certains furent internés dans les camps de concentration. La caractéristique de ces maladies est d'être de type psychosomatique.

On appelle pathologie psychosomatique l'ensemble des désordres organiques dont l'éclosion est favorisée par une situation conflictuel- 
le 33. Psychosomatique, car le déterminisme est d'origine psychique. Cette pathologie est considérée comme une façon pour l'organisme de répondre, c'est-à-dire de s'adapter au conflit auquel il est confronté, le trouble étant à la [280] fois symptôme et guérison. Plus précisément on s'accorde à dire que l'organisme (encore une fois il s'agit de l'unité cortico-viscérale, psychosomatique des Anciens) dépasse le conflit par des voies mauvaises, mais somme toute économiques. C'est le moindre mal que l'organisme choisit pour éviter la catastrophe.

Dans l'ensemble, cette pathologie est très bien connue aujourd'hui, quoique les différentes méthodes thérapeutiques proposées (relaxation, suggestion) nous paraissent très aléatoires. Pendant la Seconde Guerre mondiale, en Angleterre au cours de bombardements et en Union soviétique chez les populations assiégées notamment à Stalingrad, les descriptions de troubles survenus se sont multipliées. Actuellement, on sait parfaitement qu'il n'est pas besoin d'être blessé par balle pour souffrir dans son corps comme dans son cerveau de l'existence de la guerre. Comme toute guerre, la guerre d'Algérie a créé son contingent de maladies cortico-viscérales. Si l'on excepte le groupe $g$ ci-dessous, tous les troubles rencontrés en Algérie ont été décrits à l'occasion de guerres « classiques». Le groupe g nous a paru spécifique de la guerre coloniale d'Algérie. Cette forme particulière de pathologie (la contracture musculaire généralisée) avait déjà retenu l'attention avant le déclenchement de la Révolution. Mais les médecins qui la décrivaient en faisaient un stigmate congénital de l'indigène, une originalité (?) de son système nerveux où l'on affirmait retrouver la preuve d'une prédominance chez le colonisé du système extrapyramidal ${ }^{34}$. Cette contracture en réalité est tout simplement

33 Cette appellation qui exprime une conception idéaliste est de plus en plus abandonnée. Effectivement, la terminologie cortico-viscérale héritée des travaux soviétiques - surtout de Pavlov - a du moins l'avantage de remettre le cerveau à sa place, c'est-à-dire de le considérer comme la matrice où s'élabore précisément le psychisme.

34 Plus on est élevé sur le plan neurologique, moins on est extra-pyramidal. Comme on le voit, tout semblait concorder. 
l'accompagnement postural, l'existence dans les muscles du colonisé de sa rigidité, de sa réticence, de son refus face à l'autorité coloniale.

[281]

\section{TABLEAUX PSYCHIATRIQUES RENCONTRÉS}

\section{a) Ulcères d'estomac}

Très nombreux. Les douleurs sont à prédominance nocturne, avec vomissements importants, amaigrissement, tristesse et morosité, l'irritabilité étant l'exception. À signaler que la majorité de ces malades sont très jeunes: de 18 à 25 ans. En règle générale, nous ne conseillons jamais l'intervention chirurgicale. Deux fois une gastrectomie fut pratiquée. Il fallut dans ces deux cas réintervenir dans la même année.

\section{b) Coliques néphrétiques}

Ici encore nous trouvons des douleurs à paroxysme nocturne. Évidemment, il n'y a presque jamais de calculs. Ces coliques peuvent survenir, ce qui est rare, chez des sujets de 14 à 16 ans.

\section{c) Troubles des règles chez les femmes}

Cette pathologie est très connue, et nous ne nous y attarderons pas. Soit que les femmes restent trois à quatre mois sans règles, soit que des douleurs importantes se répercutant sur le caractère et sur le comportement accompagnent ces règles.

\section{d) Hypersomnies par tremblements idiopathiques}

Il s'agit d'adultes jeunes, à qui tout repos est interdit à cause d'un tremblement généralisé, menu, évoquant un Parkinson total. Là encore, 
des «esprits scientifiques» pourraient évoquer un déterminisme extra-pyramidal.

\section{e) Blanchissement précoce des cheveux}

Chez les rescapés de centres d'interrogatoire, les cheveux blanchissent subitement, par plaques, par régions ou totalement. Très souvent ces troubles s'accompagnent d'asthénie profonde avec désintérêt et d'impuissance sexuelle.

\section{f) Tachycardies paroxystiques}

Le rythme cardiaque brusquement s'accélère: 120, 130, 140 à la minute. Ces tachycardies s'accompagnent d'angoisse, d'impression [282] de mort imminente, et la fin de la crise est marquée par une importante sudation.

\section{g) Contracture généralisée, raideur musculaire}

Il s'agit de malades de sexe masculin qui éprouvent progressivement (dans deux cas l'apparition est brutale) de la difficulté à l'exécution de certains mouvements: monter les escaliers, marcher vite, courir. La cause de cette difficulté réside dans une rigidité caractéristique qui évoque irrésistiblement une atteinte de certaines régions du cerveau (noyaux gris centraux). C'est une rigidité en extension et la démarche se fait à petits pas. La flexion passive des membres inférieurs est presque impossible. Aucune détente ne peut être obtenue. D'emblée contracturé, incapable du moindre relâchement volontaire, le malade semble fait d'une pièce. Le visage est fixe, mais exprime un degré marqué de désorientation.

Le malade ne semble pas pouvoir « démobiliser ses nerfs». Il est constamment tendu, en attente, entre la vie et la mort. Ainsi que nous 
le disait l'un d'eux: «Vous voyez, je suis déjà raide comme un mort ${ }^{35}$. »

35 Il est superflu d'ajouter qu'il ne s'agit pas ici de contracture hystérique. 
[283]

\title{
LES DAMNÉS DE LA TERRE
}

\author{
$\mathbf{V}$ \\ De l'impulsivité criminelle \\ du Nord-Africain à la guerre \\ de Libération nationale
}

\section{Retour à la table des matières}

Il ne faut pas seulement combattre pour la liberté de son peuple. Il faut aussi pendant tout le temps que dure le combat réapprendre à ce peuple et d'abord réapprendre à soi-même la dimension de l'homme. Il faut remonter les chemins de l'histoire, de l'histoire de l'homme damné par les hommes, et provoquer, rendre possible la rencontre de son peuple et des autres hommes.

En réalité le militant qui est engagé dans un combat armé, dans une lutte nationale, a l'intention de mesurer au jour le jour toutes les dégradations infligées à l'homme par l'oppression coloniale. Le militant a quelquefois l'impression harassante qu'il lui faut ramener tout son peuple, le remonter du puits, de la grotte. Le militant s'aperçoit très souvent qu'il lui faut non seulement faire la chasse aux forces ennemies mais aussi aux noyaux de désespoir cristallisés dans le corps du coloni- 
sé. La période d'oppression est douloureuse, mais le combat, en réhabilitant l'homme opprimé, développe un processus de réintégration qui est extrêmement fécond et décisif. Le combat victorieux d'un peuple ne consacre pas uniquement le triomphe de ses droits. Il procure à ce peuple densité, cohérence et homogénéité. Car le colonialisme n'a pas fait que dépersonnaliser le colonisé. Cette dépersonnalisation est ressentie également sur le plan collectif au niveau des structures sociales. Le peuple colonisé se trouve alors réduit à un ensemble d'individus qui ne tirent leur fondement que de la présence du colonisateur.

[284] Le combat que mène un peuple pour sa libération le conduit selon les circonstances soit à rejeter, soit à faire exploser les prétendues vérités installées dans sa conscience par l'administration civile coloniale, l'occupation militaire, l'exploitation économique. Et, seul, le combat peut réellement exorciser ces mensonges sur l'homme qui infériorisent et littéralement mutilent les plus conscients d'entre nous.

Combien de fois, à Paris ou à Aix, à Alger ou à Basse-Terre, avonsnous vu des colonisés protester avec violence contre la prétendue paresse du Noir, de l'Algérien, du Vietnamien. Et pourtant n'est-il point vrai qu'en régime colonial un fellah ardent au travail, un nègre qui refuserait le repos seraient tout simplement des individualités pathologiques. La paresse du colonisé c'est le sabotage conscient de la machine coloniale: c'est, sur le plan biologique, un système d'autoprotection remarquable et c'est en tout cas un retard certain apporté à la mainmise de l'occupant sur le pays global.

La résistance des forêts et des marécages à la pénétration étrangère est l'alliée naturelle du colonisé. Il fallait le comprendre et cesser d'argumenter et d'affirmer que le nègre est un gros travailleur et le bicot un défricheur exceptionnel. En régime colonial, la vérité du bicot, la vérité du nègre, c'est de ne pas bouger le petit doigt, de ne pas aider l'oppresseur à mieux s'enfoncer dans sa proie. Le devoir du colonisé qui n'a pas encore mûri sa conscience politique et décidé de rejeter l'oppression est de se faire littéralement arracher le moindre geste. C'est là une manifestation très concrète de la non-coopération, en tout cas d'une coopération a minima. 
Ces remarques qui s'appliquent aux rapports du colonisé et du travail pourraient également s'appliquer au respect du colonisé pour les lois de l'oppresseur, au paiement régulier des impôts et des taxes, aux rapports du colonisé et du système colonial. En régime colonial, la gratitude, la sincérité, l'honneur sont des mots vides. Au cours de ces dernières années j'ai eu l'occasion de vérifier une donnée très classique : l'honneur, la dignité, le respect de la parole donnée ne peuvent se manifester que dans [285] le cadre d'une homogénéité nationale et internationale. Dès lors que vous et vos semblables êtes liquidés comme des chiens, il ne vous reste plus qu'à utiliser tous les moyens pour rétablir votre poids d'homme. Il vous faut donc peser le plus lourdement possible sur le corps de votre tortionnaire pour que son esprit égaré quelque part retrouve enfin sa dimension universelle. Au cours de ces dernières années, j'ai eu l'occasion de voir que dans l'Algérie combattante l'honneur, le don de soi, l'amour de la vie, le mépris de la mort pouvaient revêtir des formes extraordinaires. Non, il n'est pas question de chanter les combattants. Il s'agit ici d'une constatation banale que les plus enragés des colonialistes n'ont pas manqué de faire: le combattant algérien a une façon inhabituelle de se battre et de mourir et nulle référence à l'islam ou au Paradis promis ne peut expliquer cette générosité de soi quand il s'agit de protéger le peuple ou de couvrir les frères. Et ce silence écrasant - le corps bien sûr crie - et ce silence qui écrase le tortionnaire. Nous retrouvons ici, disons-nous, la très ancienne loi qui interdit à un élément quelconque de l'existence de rester immobile quand la nation se met en marche, quand l'homme revendique et affirme en même temps son humanité illimitée.

Parmi les caractéristiques du peuple algérien telles que le colonialisme les avait établies nous retiendrons sa criminalité effarante. Avant 1954, les magistrats, les policiers, les avocats, les journalistes, les médecins légistes convenaient de façon unanime que la criminalité de l'Algérien faisait problème. L'Algérien, affirmait-on, est un criminel-né. Une théorie fut élaborée, des preuves scientifiques apportées. Cette théorie fut l'objet pendant plus de 20 ans d'un enseignement universitaire. Des Algériens étudiants en médecine reçurent cet en- 
seignement et petit à petit, imperceptiblement, après s'être accommodés du colonialisme, les élites s'accommodèrent des tares naturelles du peuple algérien. Fainéants-nés, menteurs-nés, voleurs-nés, criminels-nés.

Nous nous proposons d'exposer ici cette théorie officielle, d'en rappeler les bases concrètes et l'argumentation scientifique. [286] Dans un deuxième temps nous reprendrons les faits et tâcherons de les réinterpréter.

L'Algérien tue fréquemment. C'est un fait, vous diront les magistrats, que les quatre cinquièmes des affaires instruites ont trait aux coups et blessures. Le taux de la criminalité en Algérie est l'un des plus importants, l'un des plus élevés du monde, affirment-ils. Il n'y a pas de petits délinquants. Quand l'Algérien, et cela s'applique à tous les Nord-Africains, se met hors la loi, c'est toujours au maximum.

L'Algérien tue sauvagement. Et d'abord l'arme préférée est le couteau. Les magistrats « qui connaissent le pays» se sont créé une petite philosophie sur ce sujet. Les Kabyles par exemple préfèrent le pistolet ou le fusil. Les Arabes de la plaine ont une prédilection pour le couteau. Certains magistrats se demandent si pour l'Algérien il n'y a pas exigence de voir le sang. L'Algérien, vous dira-t-on, a besoin de sentir le chaud du sang, de baigner dans le sang de la victime. Ces magistrats, ces policiers, ces médecins, dissertent très sérieusement sur les rapports de l'âme musulmane et du sang ${ }^{36}$. Un certain nombre de magistrats vont jusqu'à dire que pour l'Algérien tuer un homme, c'est d'abord et surtout l'égorger. La sauvagerie de l'Algérien se manifeste surtout par la multiplicité des blessures, l'inutilité de certaines d'entre elles portées après la mort. Les autopsies établissent incontestablement cette chose: le meurtrier donne l'impression, par l'égale gravité des blessures portées, qu'il a voulu tuer un nombre incalculable de fois.

36 On sait en effet que l'islam fait obligation de ne pas consommer la viande sans s'être assuré que l'animal a été vidé de son sang. C'est pour quoi les bêtes sont égorgées. 
L'Algérien tue pour rien. Très souvent magistrats et policiers demeurent interdits devant les motifs du meurtre: un geste, une allusion, un propos ambigu, une altercation autour d'un olivier possédé en commun, une bête qui s'aventure dans un huitième d'hectare... Devant ce meurtre, quelquefois devant ce double ou ce triple meurtre, la cause recherchée, le motif dont on attend [287] qu'il justifie et fonde ces meurtres se trouve être d'une banalité désespérante. D'où l'impression souvent que le groupe social cache les véritables motifs.

Enfin, le vol pratiqué par un Algérien se fait toujours par effraction accompagnée ou non d'assassinat, et tout cas d'agression contre le propriétaire.

Tous ces éléments réunis en faisceau autour de la criminalité algérienne ont paru suffisamment spécifier la chose pour qu'une tentative de systématisation fût édifiée.

Des observations similaires quoique moins prégnantes ayant été faites en Tunisie et au Maroc, il fut de plus en plus question de la criminalité nord-africaine. Pendant plus de 30 ans, sous la direction constante du professeur Porot, professeur de psychiatrie à la Faculté d'Alger, plusieurs équipes vont préciser les modalités d'expression de cette criminalité et en proposer une interprétation sociologique, fonctionnelle, anatomique.

Nous utiliserons ici les principaux travaux consacrés à cette question par l'école psychiatrique de la Faculté d'Alger. Les conclusions des recherches entreprises pendant plus de 20 ans furent l'objet, rappelons-le, de cours magistraux à la chaire de psychiatrie.

C'est ainsi que les médecins algériens diplômés de la Faculté d'Alger durent entendre et apprendre que l'Algérien est un criminel-né. Davantage, il me souvient de tel d'entre nous qui très sérieusement exposait ces théories apprises. Et d'ajouter: "C'est dur à avaler mais c'est scientifiquement établi. »

Le Nord-Africain est un criminel, son instinct prédateur est connu, son agressivité massive perceptible à vue d'œil. Le Nord-Africain aime les extrêmes, aussi ne peut-on jamais lui faire intégralement confian- 
ce. Aujourd'hui le plus ami, demain le plus ennemi. Imperméable aux nuances, le cartésianisme lui est fondamentalement étranger, le sens de l'équilibre, du pondéré, de la mesure heurte ses dispositions les plus intimes. Le Nord-Africain est un violent, héréditairement violent. Il y a chez lui une impossibilité à se discipliner, à canaliser ses impulsions. Oui, l'Algérien est un impulsif congénital.

[288]

Mais, précise-t-on, cette impulsivité est fortement agressive et généralement homicide. C'est ainsi que l'on arrive à expliquer le comportement non orthodoxe du mélancolique algérien. Les psychiatres français en Algérie se sont trouvés en face d'un problème difficile. Ils étaient habitués, en présence d'un malade atteint de mélancolie, à craindre le suicide. Or le mélancolique algérien tue. Cette maladie de la conscience morale qui s'accompagne toujours d'auto-accusation et de tendances autodestructrices revêt chez l'Algérien des formes hétérodestructrices. L'Algérien mélancolique ne se suicide pas. Il tue. C'est la mélancolie homicide bien étudiée par le professeur Porot dans la thèse de son élève Monserrat.

Comment l'école algérienne rend-elle compte de cette anomalie? D'abord, dit l'école d'Alger, se tuer, c'est revenir sur soi, c'est se regarder, c'est pratiquer l'introspection. Or l'Algérien est rebelle à la vie intérieure. Il n'y a pas de vie intérieure chez le Nord-Africain. Le Nord-Africain au contraire se débarrasse de ses soucis en se jetant sur l'entourage. Il n'analyse pas. La mélancolie étant par définition une maladie de la conscience morale, il est clair que l'Algérien ne peut donner naissance qu'à des pseudo-mélancolies puisque aussi bien la précarité de sa conscience et la fragilité de son sens moral sont bien connues. Cette incapacité de l'Algérien à analyser une situation, à organiser un panorama mental se comprend parfaitement si on se réfère aux deux ordres de causalité proposés par les auteurs français.

Et d'abord concernant les aptitudes intellectuelles. L'Algérien est un gros débile mental. Il faut, si l'on veut bien comprendre cette don- 
née, rappeler la séméiologie établie par l'école d'Alger, L'indigène, y est-il dit, présente les caractéristiques suivantes:

- pas ou presque pas d'émotivité :

- crédule et suggestible à l'extrême:

- entêtement tenace:

- puérilisme mental, moins l'esprit curieux de l'enfant occidental : [289]

- facilité des accidents et des réactions pithiatiques 37 .

L'Algérien ne perçoit pas l'ensemble. Les questions qu'il se pose concernent toujours les détails et excluent toute synthèse. Pointilliste, accroché aux objets, perdu dans le détail, insensible à l'idée, rebelle aux concepts. L'expression verbale est réduite au minimum. Le geste toujours impulsif et agressif. Incapable d'interpréter le détail à partir de l'ensemble, l'Algérien absolutise l'élément et prend la partie pour le tout. Aussi aura-t-il des réactions globales face à des incitations parcellaires, à des insignifiances telles un figuier, un geste, un mouton sur son terrain. L'agressivité congénitale se cherche des voies, se contente du moindre prétexte. C'est une agressivité à l'état pur 38.

Abandonnant le stade descriptif, l'école d'Alger aborde le plan explicatif. C'est en 1935 au Congrès des aliénistes et neurologistes de langue française qui se tenait à Bruxelles que le professeur Porot devait définir les bases scientifiques de sa théorie. Discutant le rapport de Baruk sur l'hystérie, il signalait que «l'indigène nord-africain, dont les activités supérieures et corticales sont peu évoluées, est un être primitif dont la vie essentiellement végétative et instinctive est surtout réglée par son diencéphale ».

37 Professeur A. Porot, Annales médico-psychologiques, 1918.

38 Dans la bouche du doyen des juges d'une chambre d'Alger cette agressivité de l'Algérien se traduit par son amour de la «fantasia». « Toute cette révolte, disait-il en 1955, on a tort de la croire politique. De temps à autre, il faut que ça sorte cet amour du baroud qu'ils ont! » Pour l'ethnologue, établir une série de tests et de jeux projectifs susceptibles de canaliser les instincts agressifs globaux de l'indigène aurait pu en 1955-1956 stopper la révolution dans les Aurès. 
Pour bien mesurer l'importance de cette découverte du professeur Porot il faut rappeler que la caractéristique de l'espèce humaine, quand on la compare aux autres vertébrés, est la corticalisation. Le diencéphale est l'une des parties les plus primitives du cerveau et l'homme est d'abord le vertébré où domine le cortex.

[290]

Pour le professeur Porot, la vie de l'indigène nord-africain est dominée par les instances diencéphaliques. Cela revient à dire que l'indigène nord-africain d'une certaine façon est privé de cortex. Le professeur Porot n'évite pas cette contradiction et en avril 1939 dans le Sud médical et chirurgicalil précise, en collaboration avec son élève Sutter actuellement professeur de psychiatrie à Alger : «Le primitivisme n'est pas un manque de maturité, un arrêt marqué dans le développement du psychisme intellectuel. Il est une condition sociale parvenue au terme de son évolution, il est adapté de façon logique à une vie différente de la nôtre. " Enfin, les professeurs abordent la base même de la doctrine: «Ce primitivisme n'est pas seulement une manière résultant d'une éducation spéciale, il a des assises beaucoup plus profondes et nous pensons même qu'il doit avoir son substratum dans une disposition particulière de l'architectonie, du moins de la hiérarchisation dynamique des centres nerveux. " Comme on le voit, l'impulsivité de l'Algérien, la fréquence et les caractères de ses meurtres, ses tendances permanentes à la délinquance, son primitivisme ne sont pas un hasard. Nous sommes en présence d'un comportement cohérent, d'une vie cohérente scientifiquement explicable. L'Algérien n'a pas de cortex, ou pour être plus précis, la domination comme chez les vertébrés inférieurs est diencéphalique. Les fonctions corticales, si elles existent, sont très fragiles, pratiquement non intégrées dans la dynamique de l'existence. Il n'y a donc ni mystère ni paradoxe. La réticence du colonisateur à confier une responsabilité à l'indigène n'est pas du racisme ou du paternalisme mais tout simplement une appréciation scientifique des possibilités biologiquement limitées du colonisé.

Terminons cette revue en demandant une conclusion à l'échelle de l'Afrique au docteur Carothers, expert de l'Organisation mondiale de 
la santé. Cet expert international a réuni dans un livre paru en 195439 l'essentiel de ses observations.

[291]

Le docteur Carothers pratiquait en Afrique centrale et orientale mais ses conclusions recoupent celles de l'école nord-africaine. Pour l'expert international en effet, "l'Africain utilise très peu ses lobes frontaux. Toutes les particularités de la psychiatrie africaine peuvent être rapportées à une paresse frontale 40 ».

Pour bien se faire comprendre le docteur Carothers établit une comparaison très vivante. C'est ainsi qu'il avance que l'Africain normal est un Européen lobotomisé. On sait que l'école anglo-saxonne avait cru trouver une thérapeutique radicale de certaines formes graves de maladies mentales en pratiquant l'exclusion d'une partie importante du cerveau. Les gros délabrements de la personnalité constatés ont fait depuis abandonner cette méthode. D'après le docteur Carothers, la similitude existant entre l'indigène africain normal et le lobotomisé européen est frappante.

Le docteur Carothers, après avoir étudié les travaux des différents auteurs exerçant en Afrique, nous propose une conclusion qui fonde une conception unitaire de l'Africain. «Telles sont, écrit-il, les données des cas qui ne concernent pas les catégories européennes. Elles ont été recueillies dans les différentes régions de l'Afrique Est, Ouest, Sud et dans l'ensemble chacun des auteurs n'avaient que peu ou pas de connaissance des travaux des autres. La similitude essentielle de ces travaux est donc tout à fait remarquable ${ }^{41}$. "

Signalons avant de terminer que le docteur Carothers définissait la révolte des Mau-Mau comme l'expression d'un complexe inconscient de

39 Carothers, «Psychologie normale et pathologique de l'Africain», Études ethnopsychiatriques, Masson éditeur.

40 Ibid., p. 176.

41 Ibid., p. 178. 
frustration dont la réédition pourrait être scientifiquement évitée par des adaptations psychologiques spectaculaires.

Ainsi donc un comportement inhabituel : la fréquence de la criminalité de l'Algérien, la banalité des motifs retrouvés, le caractère meurtrier et toujours hautement sanglant des rixes, [292] posait aux observateurs un problème. L'explication proposée qui est devenue matière d'enseignement semble être en dernière analyse la suivante: la disposition des structures cérébrales du Nord-Africain rend compte à la fois de la paresse de l'indigène, de son inaptitude intellectuelle et sociale et de son impulsivité quasi animale. L'impulsivité criminelle du Nord-Africain est la transcription dans l'ordre du comportement d'un certain arrangement du système nerveux. C'est une réaction neurologiquement compréhensible, inscrite dans la nature des choses, de la chose biologiquement organisée. La non-intégration des lobes frontaux dans la dynamique cérébrale explique la paresse, les crimes, les vols, les viols, le mensonge. Et la conclusion, c'est un sous-préfet - aujourd'hui préfet - qui me la donnait : « À ces êtres naturels, disait-il, qui obéissent aveuglément aux lois de leur nature, il faut opposer des cadres stricts et implacables. Il faut domestiquer la nature, non la convaincre. " Discipliner, dresser, mater et aujourd'hui pacifier sont les vocables les plus utilisés par les colonialistes dans les territoires occupés.

Si nous avons longuement repris les théories proposées par les hommes de science colonialistes, ce fut moins pour montrer leur pauvreté et leur absurdité que pour aborder un problème théorique et pratique extrêmement important. De fait, parmi les questions qui se posaient à la Révolution, parmi les sujets qui pouvaient être débattus au niveau de l'explication politique et de la démystification, la criminalité algérienne ne représentait qu'un sous-secteur. Mais précisément les entretiens qui eurent lieu autour de ce thème furent à ce point féconds qu'ils nous permirent d'approfondir et de mieux cerner la notion de libération individuelle et sociale. Lorsque dans la pratique révolutionnaire on aborde devant les cadres et les militants la question de 
la criminalité algérienne, quand on expose le nombre moyen de crimes, de délits, de vols de la période d'avant la Révolution, quand on explique que la physionomie d'un crime, la fréquence des délits sont fonction des rapports existant entre les hommes et les femmes, entre les hommes et l'État et que chacun [293] comprend; quand on assiste à vue d'œeil à la dislocation de la notion d'Algérien ou de Nord-Africain criminel par vocation, notion qui était également fichée dans la conscience de l'Algérien parce que enfin « nous sommes coléreux, bagarreurs, mauvais... c'est comme cela... », alors, oui, on peut dire que la Révolution progresse.

Le problème théorique important est qu'il faut à tout moment et en tout lieu expliciter, démystifier, pourchasser l'insulte à l'homme qui est en soi. Il ne faut pas attendre que la nation produise de nouveaux hommes. Il ne faut pas attendre qu'en perpétuel renouvellement révolutionnaire les hommes insensiblement se transforment. Il est bien vrai que ces deux processus importent mais il faut aider la conscience. La pratique révolutionnaire, si elle se veut globalement libératrice et exceptionnellement féconde exige que rien d'insolite ne subsiste. On ressent avec une particulière force la nécessité de totaliser l'événement, de tout amener avec soi, de tout régler, d'être responsable de tout. La conscience alors ne rechigne pas à revenir en arrière, à marquer le pas s'il le faut. C'est pourquoi, dans la progression sur le terrain d'une unité de combat, la fin d'une embuscade ne signifie pas le repos mais bien le moment pour la conscience de faire un bout de chemin, car tout doit aller de pair.

Oui, spontanément l'Algérien donnait raison aux magistrats et aux policiers ${ }^{42}$. Il a donc fallu prendre cette criminalité algérienne vécue

42 Il est clair d'ailleurs que cette identification à l'image produite par l'Européen était très ambivalente. L'Européen en effet semblait rendre un hommage - également ambivalent - à l'Algérien violent, passionné, brutal, jaloux, fier, orgueilleux qui joue sa vie sur un détail ou sur un mot, etc. Signalons en passant que dans les confrontations avec le Français de France, les Européens d'Algérie tendent de plus en plus à s'identifier à cette image de l'Algérien par opposition au Français. 
sur le plan du narcissisme comme manifestation de l'authentique virilité et reposer le problème sur le plan de l'histoire coloniale. Par exemple, montrer que la criminalité des Algériens en France diffère fondamentalement de la criminalité des Algériens soumis à l'exploitation directement coloniale.

[294]

Une deuxième chose devait retenir notre attention. en Algérie la criminalité algérienne se déroule pratiquement en cercle fermé. Les Algériens s'entre-volaient, s'entre-déchiraient, s'entre-tuaient. En Algérie, l'Algérien s'attaquait peu aux Français et évitait les rixes avec les Français. En France par contre l'émigré créera une criminalité intersociale, intergroupes.

En France la criminalité algérienne diminue. Elle s'adresse surtout aux Français et les mobiles en sont radicalement nouveaux. Un paradoxe nous a considérablement aidés à démystifier les militants: on constate depuis 1954, une quasi-disparition des crimes de droit commun. Plus de disputes, plus de détails insignifiants entraînant mort d'homme. Plus de colères explosives parce que le front de ma femme ou son épaule gauche ont été perçus par le voisin. La lutte nationale semble avoir canalisé toutes les colères, nationalisé tous les mouvements affectifs ou émotionnels. Cela, les juges ou les avocats français l'avaient déjà constaté mais il fallait que le militant en fût conscient, il fallait l'amener à en connaître les raisons.

Reste l'explication.

Fallait-il dire que la guerre, terrain privilégié d'expression d'une agressivité enfin socialisée, canalise vers l'occupant des gestes congénitalement meurtriers? C'est une constatation banale que les grandes secousses sociales diminuent la fréquence de la délinquance et les troubles mentaux. On pouvait donc parfaitement expliquer cette régression de la criminalité algérienne par l'existence d'une guerre qui cassait l'Algérie en deux, rejetant du côté ennemi la machine judiciaire, administrative. 
Or, dans les pays maghrébins déjà libérés ce même phénomène signalé au cours des luttes de libération se maintient et se précise avec l'indépendance. Il semble donc que le contexte colonial soit suffisamment original pour autoriser une réinterprétation de la criminalité. C'est ce que nous avons fait à l'intention des combattants. Aujourd'hui tout le monde chez nous sait que la criminalité n'est pas la conséquence du caractère congénital de l'Algérien ni de l'organisation de son système [295] nerveux. La guerre d'Algérie, les guerres de libération nationale font surgir les véritables protagonistes. Dans la situation coloniale, on l'a montré, des indigènes sont entre eux. Ils ont tendance à se servir réciproquement d'écran. Chacun cache à l'autre l'ennemi national. Et lorsque, fatigué après une dure journée de seize heures, le colonisé s'affale sur sa natte et qu'un enfant à travers la cloison de toile pleure et l'empêche de dormir, comme par hasard c'est un petit Algérien. Quand il va solliciter un peu de semoule ou un peu d'huile à l'épicier auquel il doit déjà quelques centaines de francs et qu'il se voit refuser cette faveur, une immense haine et une grosse envie de tuer le submergent et l'épicier est un Algérien. Lorsque après l'avoir évité des semaines durant il se trouve un jour coincé par le caïd qui lui réclame «des impôts», il ne lui est même pas donné le loisir de haïr l'administrateur européen: le caiid est là qui sollicite cette haine et c'est un Algérien.

Exposé à des tentatives de meurtre quotidiennes: de famine, d'expulsion de la chambre non payée, de sein maternel desséché, d'enfants squelettiques, le chantier fermé, les chômeurs qui rôdent autour du gérant comme des corbeaux, l'indigène en arrive à voir son semblable comme un ennemi implacable. S'il écorche ses pieds nus sur une grosse pierre au milieu du chemin, c'est un indigène qui l'aura placée là et les quelques olives que l'on s'apprête à cueillir, voilà que les enfants de $X$... dans la nuit les ont mangées. Oui, dans la période coloniale en Algérie et ailleurs on peut faire beaucoup de choses pour un kilo de semoule. On peut tuer plusieurs personnes. Il faut de l'imagination pour comprendre ces choses. Ou de la mémoire. Dans les camps de concentration des hommes se sont tués pour un morceau de 
pain. Je me souviens d'une scène horrible. C'était à Oran en 1944. Du camp où nous attendions l'embarquement, les militaires lançaient des morceaux de pain à de petits Algériens qui se les disputaient avec rage et haine. Les vétérinaires pourraient éclairer ces phénomènes en évoquant le fameux «peck-order» constaté dans les basses-cours. Le mais qui est distribué est en effet l'objet d'une compétition implacable. Certaines [296] volailles, les plus fortes, dévorent toutes les graines tandis que d'autres moins agressives maigrissent à vue d'œil. Toute colonie tend à devenir une immense basse-cour, un immense camp de concentration où la seule loi est celle du couteau.

En Algérie depuis la guerre de Libération nationale, tout a changé. Les réserves entières d'une famille ou d'une metcha peuvent être en une seule soirée offertes à une compagnie de passage. Le seul âne de la famille peut être prêté pour assurer le transport d'un blessé. Et quand, plusieurs jours après, le propriétaire apprendra la mort de son animal mitraillé par un avion, il ne se lancera pas en imprécations et en menaces. Il ne mettra pas en doute la mort de son animal, mais il demandera, inquiet, si le blessé est sain et sauf.

En régime colonial, on peut tout faire pour un kilo de pain ou un misérable mouton... Les rapports de l'homme avec la matière, avec le monde, avec l'histoire, sont, en période coloniale, des rapports avec la nourriture. Pour un colonisé, dans un contexte d'oppression comme celui de l'Algérie, vivre ce n'est point incarner des valeurs, s'insérer dans le développement cohérent et fécond d'un monde. Vivre c'est ne pas mourir. Exister c'est maintenir la vie. Chaque datte est une victoire. Non un résultat du labeur, mais une victoire ressentie comme triomphe de la vie. Aussi dérober les dattes, permettre à son mouton de manger l'herbe du voisin ne sont pas négation de la propriété d'autrui, transgression d'une loi ou irrespect. Ce sont des tentatives de meurtres. Il faut avoir vu en Kabylie des hommes et des femmes des semaines durant aller chercher de la terre au fond de la vallée et la remonter par petits paniers pour comprendre qu'un vol est une tentative de meurtre et non un geste inamical ou illégal. C'est que la seule perspective est cet estomac de plus en plus rétréci, de moins en moins exigeant cer- 
tes, mais qu'il faut tout de même contenter. À qui s'en prendre? Le Français est dans la plaine avec les policiers, l'armée, et les tanks. Sur la montagne il n'y a que des Algériens. Là-haut le ciel avec ses promesses d'outre-tombe, en bas les Français avec leurs promesses bien concrètes de prison, de [297] matraquage, d'exécutions. Forcément, on bute sur soi-même. On découvre ici le noyau de cette haine de soimême qui caractérise les conflits raciaux dans les sociétés ségréguées.

La criminalité de l'Algérien, son impulsivité, la violence de ses meurtres ne sont donc pas la conséquence d'une organisation du système nerveux ni d'une originalité caractérielle mais le produit direct de la situation coloniale. Que les combattants algériens aient discuté ce problème, qu'ils n'aient pas craint de remettre en cause les croyances installées en eux par le colonialisme, qu'ils aient compris que chacun était l'écran de l'autre et qu'en réalité chacun se suicidait en se jetant sur l'autre devait avoir une importance primordiale dans la conscience révolutionnaire. Encore une fois, l'objectif du colonisé qui se bat est de provoquer la fin de la domination. Mais il doit également veiller à la liquidation de toutes les non-vérités fichées dans son corps par l'oppression. Dans un régime colonial tel qu'il existait en Algérie, les idées professées par le colonialisme n'influençaient pas seulement la minorité européenne mais aussi l'Algérien. La libération totale est celle qui concerne tous les secteurs de la personnalité. L'embuscade ou l'accrochage, la torture ou le massacre de ses frères enracinent la détermination de vaincre, renouvellent l'inconscient et alimentent l'imagination. Quand la nation démarre en totalité, l'homme nouveau n'est pas une production a posteriori de cette nation mais coexiste avec elle, se développe avec elle, triomphe avec elle. Cette exigence dialectique explique la réticence à l'égard des colonisations adaptées et des réformes de façade. L'indépendance n'est pas un mot à exorciser mais une condition indispensable à l'existence des hommes et des femmes vraiment libérés, c'est-à-dire maîtres de tous les moyens matériels qui rendent possible la transformation radicale de la société. 
[301]

\section{LES DAMNÉS DE LA TERRE}

\section{CONCLUSION}

\section{Retour à la table des matières}

Allons, camarades, il vaut mieux décider dès maintenant de changer de bord. La grande nuit dans laquelle nous fûmes plongés, il nous faut la secouer et en sortir. Le jour nouveau qui déjà se lève doit nous trouver fermes, avisés et résolus.

Il nous faut quitter nos rêves, abandonner nos vieilles croyances et nos amitiés d'avant la vie. Ne perdons pas de temps en stériles litanies ou en mimétismes nauséabonds. Quittons cette Europe qui n'en finit pas de parler de l'homme tout en le massacrant partout où elle le rencontre, à tous les coins de ses propres rues, à tous les coins du monde.

Voici des siècles que l'Europe a stoppé la progression des autres hommes et les a asservis à ses desseins et à sa gloire; des siècles qu'au nom d'une prétendue «aventure spirituelle» elle étouffe la quasi-totalité de l'humanité. Regardez-la aujourd'hui basculer entre la désintégration atomique et la désintégration spirituelle.

Et pourtant, chez elle, sur le plan des réalisations on peut dire qu'elle a tout réussi. 
L'Europe a pris la direction du monde avec ardeur, cynisme et violence. Et voyez combien l'ombre de ses monuments s'étend et se multiplie. Chaque mouvement de l'Europe a fait craquer les limites de l'espace et celles de la pensée. L'Europe s'est refusée à toute humilité, à toute modestie, mais aussi à toute sollicitude, à toute tendresse.

Elle ne s'est montrée parcimonieuse qu'avec l'homme, mesquine, carnassière homicide qu'avec l'homme.

Alors, frères, comment ne pas comprendre que nous avons mieux à faire que de suivre cette Europe-là.

[302]

Cette Europe qui jamais ne cessa de parler de l'homme, jamais de proclamer qu'elle n'était inquiète que de l'homme, nous savons aujourd'hui de quelles souffrances l'humanité a payé chacune des victoires de son esprit.

Allons, camarades, le jeu européen est définitivement terminé, il faut trouver autre chose. Nous pouvons tout faire aujourd'hui à condition de ne pas singer l'Europe, à condition de ne pas être obsédés par le désir de rattraper l'Europe.

L'Europe a acquis une telle vitesse, folle et désordonnée, qu'elle échappe aujourd'hui à tout conducteur, à toute raison et qu'elle va dans un vertige effroyable vers des abîmes dont il vaut mieux le plus rapidement s'éloigner.

Il est bien vrai cependant qu'il nous faut un modèle, des schèmes, des exemples. Pour beaucoup d'entre nous, le modèle européen est le plus exaltant. Or, on a vu dans les pages précédentes à quelles déconvenues nous conduisait cette imitation. Les réalisations européennes, la technique européenne, le style européen doivent cesser de nous tenter et de nous déséquilibrer.

Quand je cherche l'homme dans la technique et dans le style européens, je vois une succession de négations de l'homme, une avalanche de meurtres. 
La condition humaine, les projets de l'homme, la collaboration entre les hommes pour des tâches qui augmentent la totalité de l'homme sont des problèmes neufs qui exigent de véritables inventions.

Décidons de ne pas imiter l'Europe et bandons nos muscles et nos cerveaux dans une direction nouvelle. Tâchons d'inventer l'homme total que l'Europe a été incapable de faire triompher.

Il y a deux siècles, une ancienne colonie européenne s'est mis en thte de rattraper l'Europe. Elle y a tellement réussi que les États-Unis d'Amérique sont devenus un monstre où les tares, les maladies et l'inhumanité de l'Europe ont atteint des dimensions épouvantables.

Camarades, n'avons-nous pas autre chose à faire que de créer une troisième Europe? L'Occident a voulu être une aventure de l'Esprit. C'est au nom de l'Esprit, de l'esprit européen [303] s'entend, que l'Europe a justifié ses crimes et légitimité l'esclavage dans lequel elle maintenait les quatre cinquièmes de l'humanité.

Oui, l'esprit européen a eu de singuliers fondements. Toute la réflexion européenne s'est déroulée dans des lieux de plus en plus désertiques, de plus en plus escarpés. On prit ainsi l'habitude d'y rencontrer de moins en moins l'homme.

Un dialogue permanent avec soi-même, un narcissisme de plus en plus obscène n'ont cessé de faire le lit à un quasi-délire où le travail cérébral devient une souffrance, les réalités n'étant point celles de I'homme vivant, travaillant et se fabriquant mais des mots, des assemblages divers de mots, les tensions nées des significations contenues dans les mots. Il s'est cependant trouvé des Européens pour convier les travailleurs européens à briser ce narcissisme et à rompre avec cette déréalisation.

D'une manière générale, les travailleurs européens n'ont pas répondu à ces appels. C'est que les travailleurs se sont crus, eux aussi, concernés par l'aventure prodigieuse de l'Esprit européen.

Tous les éléments d'une solution aux grands problèmes de l'humanité ont, à des moments différents, existé dans la pensée de 
l'Europe. Mais l'action des hommes européens n'a pas réalisé la mission qui lui revenait et qui consistait à peser avec violence sur ces éléments, à modifier leur arrangement, leur être, à les changer, enfin à porter le problème de l'homme à un niveau incomparablement supérieur.

Aujourd'hui, nous assistons à une stase de l'Europe. Fuyons, camarades, ce mouvement immobile où la dialectique, petit à petit, s'est muée en logique de l'équilibre. Reprenons la question de l'homme. Reprenons la question de la réalité cérébrale, de la masse cérébrale de toute l'humanité dont il faut multiplier les connexions, diversifier les réseaux et réhumaniser les messages.

Allons frères, nous avons beaucoup trop de travail pour nous amuser des jeux d'arrière-garde. L'Europe a fait ce qu'elle devait faire et somme toute elle l'a bien fait; cessons de l'accuser mais [304] disonslui fermement qu'elle ne doit plus continuer à faire tant de bruit. Nous n'avons plus à la craindre, cessons donc de l'envier.

Le tiers monde est aujourd'hui en face de l'Europe comme une masse colossale dont le projet doit être d'essayer de résoudre les problèmes auxquels cette Europe n'a pas su apporter de solutions.

Mais, alors, il importe de ne point parler rendement, de ne point parler intensification, de ne point parler rythmes. Non, il ne s'agit pas de retour à la Nature. Il s'agit très concrètement de ne pas tirer les hommes dans des directions qui les mutilent, de ne pas imposer au cerveau des rythmes qui rapidement l'oblitèrent et le détraquent. Il ne faut pas, sous le prétexte de rattraper, bousculer l'homme, l'arracher de lui-même, de son intimité, le briser, le tuer.

Non, nous ne voulons rattraper personne. Mais nous voulons marcher tout le temps, la nuit et le jour, en compagnie de l'homme, de tous les hommes. Il s'agit de ne pas étirer la caravane, car alors chaque rang perçoit à peine celui qui le précède et les hommes qui ne se reconnaissent plus se rencontrent de moins en moins, se parlent de moins en moins. 
Il s'agit pour le tiers monde de recommencer une histoire de I'homme qui tienne compte à la fois des thèses quelquefois prodigieuses soutenues par l'Europe mais aussi des crimes de l'Europe dont le plus odieux aura été, au sein de l'homme, l'écartèlement pathologique de ses fonctions et l'émiettement de son unité, dans le cadre d'une collectivité la brisure, la stratification, les tensions sanglantes alimentées par des classes, enfin, à l'échelle immense de l'humanité, les haines raciales, l'esclavage, l'exploitation et surtout le génocide exsangue que constitue la mise à l'écart d'un milliard et demi d'hommes.

Donc, camarades, ne payons pas de tribut à l'Europe en créant des États, des institutions et des sociétés qui s'en inspirent.

L'humanité attend autre chose de nous que cette imitation caricaturale et dans l'ensemble obscène.

[305]

Si nous voulons transformer l'Afrique en une nouvelle Europe, l'Amérique en une nouvelle Europe, alors confions à des Européens les destinées de nos pays. Ils sauront mieux faire que les mieux doués d'entre nous.

Mais si nous voulons que l'humanité avance d'un cran, si nous voulons la porter à un niveau différent de celui où l'Europe l'a manifestée, alors il faut inventer, il faut découvrir.

Si nous voulons répondre à l'attente de nos peuples, il faut chercher ailleurs qu'en Europe

Davantage, si nous voulons répondre à l'attente des Européens, il ne faut pas leur renvoyer une image, même idéale, de leur société et de leur pensée pour lesquelles ils éprouvent épisodiquement une immense nausée.

Pour l'Europe, pour nous-mêmes et pour l'humanité, camarades, il faut faire peau neuve, développer une pensée neuve, tenter de mettre sur pied un homme neuf. 
[307]

\author{
LES DAMNÉS DE LA TERRE \\ POSTFACE \\ à l'édition de 2002
}

par Mohammed Harbi

\title{
Retour à la table des matières
}

Nous serions-nous trompés, nous qui pensions que le capitalisme n'était pas un horizon indépassable? Le socialisme bureaucratique a vécu. Le tiers-mondisme aussi. Leur défaite, précédée de tragédies multiples et d'une dépréciation inédite de la vie humaine, arrange tous ceux qui, en Occident, ont tremblé pour leurs privilèges quand, dans les années soixante, l'initiative est passée du côté des peuples opprimés.

Contraintes et forcées, les grandes puissances industrielles ont alors dû céder du terrain face aux revendications des pays du tiers monde. Depuis, les évolutions récentes les ont confortées dans la certitude qu'il n'est d'autre voie que la leur pour sortir de la nuit et accéder au développement et à la démocratie. Mais dans les faits, elles comptent davantage sur les pressions économiques et idéologiques et sur les institutions financières internationales qu'elles dominent, le FMI et la Banque mondiale. 
Reste que la profondeur de la crise du tiers monde nécessite un bilan critique de la période postcoloniale et des idéologies qui ont accompagné ou impulsé le mouvement d'émancipation. Dans cette perspective, la réévaluation de l'œuvre de Frantz Fanon, et notamment Les Damnés de la terre, prend une importance d'autant plus grande que nombre d'idées reçues sur la dynamique révolutionnaire trouvent leur origine dans une vision de l'expérience algérienne - l'une des sources de sa réflexion dans ce livre-testament - qui sera souvent déformée.

Une des raisons de la force du «fanonisme » est qu'il répondait à l'aspiration des «élites anti-élitistes» du tiers monde de disposer d'une formulation des lois du développement social différente de celle que le marxisme dégradé des staliniens diffusait [308] à l'époque. Fanon défendait le tiers monde par son action et par la connaissance qu'il avait de ses ennemis extérieurs et intérieurs. Je l'ai connu en Tunisie en mai 1959, où nous collaborions tous deux à des instances du Gouvernement provisoire de la République algérienne (GPRA). Nous avions en commun une interprétation sociale des événements révolutionnaires et le rejet des rationalisations désincarnées sur le rôle d'avant-garde des intellectuels. Nos désaccords portaient sur le rôle moteur qu'il accordait à la paysannerie, sur sa croyance en une conscience nationale unanime et son approche du phénomène religieux. Sur ce dernier point, Fanon ne pouvait, par situation - il vivait au contact de militants plutôt agnostiques -, mesurer la place dérisoire que la pensée des Lumières occupait dans les espaces culturels algériens. Cette pensée n'était qu'un petit affluent du fleuve qui était à l'origine de l'adhésion au FLN d'une majorité plus sensible à l'influence de la religion.

Mais cela ne retire rien à l'importance des Damnés de la terre. Tant dans les parties qui font problème que dans celles qui ont une valeur prémonitoire, ce livre nous permet d'engager un débat en profondeur sur le devenir de l'idéal révolutionnaire et de ses retombées actuelles. «Du passé, faisons table rase»: cette idée de L'Internationale, Fanon l'a faite sienne. Et on ne peut comprendre sa problématique en faisant l'impasse sur l'apport de Marx. 
C'est avec Marx que prend forme une problématique finalisée et universelle de la révolution. Son analyse de la société capitaliste, fondamentalement européenne, de son temps n'envisage d'alternative qu'entre le capitalisme et le communisme, avec comme acteurs la bourgeoisie pour le premier, le prolétariat pour le second. Les autres classes de la société sont soumises à la dynamique de leur conflit. Le prolétariat est érigé en sujet de l'histoire: porteur de l'universel, il est la force qui, en se libérant de ses chaînes, libère l'humanité. Dans cette analyse, les colonisés ne sont pas au centre de la dynamique historique. (Et, au fond, lorsque le Parti communiste français soumettait le sort des pays coloniaux à l'avenir de l'« Union française», il [309] était dans cette logique : c'était la révolution anticapitaliste de la France qui devait ouvrir la voie de la libération aux colonisés, alliés subordonnés du prolétariat français.)

En Russie, les populistes plaident le principe de la différence russe et contestent les thèses de Marx : ils estiment que la révolution, dans leur pays, sera plutôt paysanne que prolétarienne et évitera l'étape bourgeoise de développement capitaliste pour amener directement au socialisme. Face à cette objection, Marx introduit (dans une correspondance avec les marxistes russes Plekhanov et Vera Zassoulitch) une retouche à son schéma et concède que si la révolution russe donne le coup d'envoi à la révolution prolétarienne en Occident, elle pourra s'accomplir sans que la société russe, alors largement rurale et traditionnelle, passe par l'étape capitaliste. Après la mort de Marx, son compagnon Friedrich Engels revient sur l'idée d'un exceptionnalisme russe: seuls le prolétariat et la paysannerie sont des classes révolutionnaires.

Un tournant dans la formulation du modèle révolutionnaire s'opère avec Lénine : étendant à l'échelle mondiale le principe de la lutte des classes, il continue dans la voie tracée par Marx à inscrire la révolution dans un projet mondial, mais considère qu'elle peut intervenir dans le maillon faible de l'impérialisme, c'est-à-dire dans des sociétés à faible composante industrielle et urbaine et donc à majorité paysanne. Le sujet messianique reste le prolétariat, mais ses intérêts sont pris en 
charge par un parti, parce que, pense Lénine, livré à lui-même, il est naturellement trade-unioniste. Ce n'était pas l'opinion de Marx. Nous sommes en présence d'une autre conception de la dynamique révolutionnaire. Lénine est un pragmatique et sa pensée est à l'affût de l'événement qui ouvre la voie à la révolution, que ce soit en Orient - où il voit mûrir les «matériaux incendiaires de la révolution mondiale» (1908) - ou en Europe.

Avec octobre 1917, c'est un pays essentiellement rural qui donne le signal de la révolution, mais l'étincelle n'engendre pas l'incendie espéré. Et c'est le socialisme dans un seul pays.

Dans l'Internationale communiste, des «tiers-mondistes» avant la lettre, le Tatar Sultan Galiev, I'Indien Roy, l'Indonésien [310] Tan Malaka attendaient que le mouvement fasse des peuples coloniaux le moteur de changement et non pas seulement un objet de sa stratégie globale, comme le souligne Maxime Rodinson 43 . Leurs efforts resteront vains mais, à travers bien des péripéties, l'idée fera son chemin, dans le mouvement communiste, avec Mao Dzédoung, et en dehors de lui avec Frantz Fanon. Tous deux mettent l'accent sur la révolution paysanne: ce n'est plus au prolétariat des pays occidentaux d'entraîner les peuples opprimés dans la lutte, c'est la lutte de ces peuples qui déterminera la cause révolutionnaire du prolétariat mondial. Cette vision fait des adeptes, y compris en Europe.

Mais à la différence de Fanon, Mao Dzédoung ne « ruralise » pas sa stratégie: le prolétariat demeure l'avant-garde, par parti communiste interposé. Cependant, Fanon innove. Il ne se contente pas d'une analyse purement économique de l'impérialisme. Il théorise aussi le conflit identitaire et culturel et tente de montrer que les vrais damnés de la terre, les exploités absolus, ce sont les colonisés. Le procès de l'Occident ne s'enferme pas dans la singularité comme chez les nationalistes: c'est l'espérance universaliste qui en est le ressort.

43 Maxime Rodinson, Marxisme et monde musulman, Le Seuil, Paris, 1972, p. 307. 
Pour autant, le plaidoyer pour la cause du tiers monde n'est pas aveugle: Fanon a conscience du décalage qui existe entre la base matérielle des pays sous domination et le projet révolutionnaire. Les pesanteurs sociologiques qui hypothèquent le développement et la démocratie ne lui échappent pas. Son expérience africaine, en Tunisie et au Ghana surtout, l'a averti des dérives qui menacent les élites politiques dans la construction des États: autoritarisme, rejet du pluralisme, corruption et personnalisation du pouvoir. Peut-être avait-il aussi comme arrière-pensée, lui, le chantre d'une nouvelle révolution mondiale, l'échec de l'expérience soviétique avec son socialisme dans un seul pays, la transformation des mouvements révolutionnaires en défenseurs de la citadelle et le despotisme d'un parti unique.

[311]

L'actualité politique de Fanon, aujourd'hui, ne s'arrête pas à ces considérations. Nul, avant lui, en Afrique, n'a su analyser les circonstances de la production des élites, leur filiation avec le colonisateur, et souligner l'« élitisme exacerbé » des catégories instruites avec leur conception de leur rôle naturel à diriger et leur discours sur l'immaturité des masses. Un des obstacles à la démocratie et non des moindres gît là.

Il serait donc injuste de ne retenir des Damnés de la terre que des thèmes lyriques discutables, le messianisme paysan, la violence rédemptrice, la constitution artificielle du tiers monde en bloc géopolitique et d'occulter les thèmes d'une actualité brûlante qui nous mettent en garde contre les dangers du romantisme révolutionnaire. Ce n'est pas dans les grandes espérances que réside une demande radicale, mais dans la vigilance sourcilleuse de la pensée critique à l'égard de tout ce qui concourt à favoriser la division en dirigeants et exécutants, de tout ce qui s'érige en bureaucratie revendiquant le savoir et le pouvoir sur des masses définies comme naturellement incompétentes et à soumettre. Ce ne serait là que la permanence de la topologie entre exploiteurs et exploités: les exploiteurs changent, mais les exploités restent les mêmes. 
Ajoutons pour finir que l'inspiration de Fanon passe aussi dans la manière dont il comprend sa pratique de psychiatre. L'institution psychiatrique lui apparaît comme une machine à normaliser le « fou » et à lui imposer des normes sans rapport avec ce dont sa parole est l'expression. Inscrit dans le mouvement antipsychiatrique, il mettra l'accent sur la libération de cette parole en renversant la relation entre le médecin et le patient. C'est du côté de la folie que se trouvent une vérité et une authenticité auxquelles il faut laisser son libre exercice. Entre le populisme à dimension libertaire de Fanon et sa pratique médicale, le parallélisme est évident.

Fin du texte 